\title{
1,3-Asymmetric Induction in Diastereoselective Allylations of Chiral N-Tosyl Imines
}

Anna Lo, David A. Gutierrez, Garrett Toth-Williams, James C. Fettinger, and Jared T. Shaw*

${ }^{\dagger}$ Department of Chemistry, University of California, One Shields Avenue, Davis, California 95616, United States

Supporting Information

\section{Table of Contents}

1. Materials and Instrumentation $\quad$ S2

2. Experimental Procedures S3

3. ${ }^{1} \mathrm{H}$ and ${ }^{13} \mathrm{C}$ NMR Spectra $\quad$ S22

4. Computational Methods $\quad$ S76

5. X-ray Crystallography Data $\quad$ S90 


\section{Materials and Instrumentation}

Unless otherwise specified, all commercially available reagents were used as received. All reactions using dried solvents were carried out under an atmosphere of argon in oven-dried glassware with magnetic stirring. Dry solvent was dispensed from a solvent purification system that passes solvent through two columns of dry neutral alumna. ${ }^{1} \mathrm{H}$ and ${ }^{13} \mathrm{C}$ NMR spectra were acquired at ambient temperature using Varian-600 (600 and $151 \mathrm{MHz}$, respectively), or Bruker-400 (400 and $100 \mathrm{MHz}$, respectively) spectrometers, as indicated. The data are reported as follows: chemical shift in ppm from internal tetramethylsilane or referenced to residual solvent $\left({ }^{1} \mathrm{H} N M R: \mathrm{CDCl}_{3} \quad \delta\right.$ 7.26. ${ }^{13} \mathrm{C} \mathrm{NMR}$ : $\mathrm{CDCl}_{3} \delta$ 77.16) on the $\delta$ scale, multiplicity (appar = apparent, $\mathrm{br}=$ broad, $\mathrm{s}=$ singlet, $\mathrm{d}=$ doublet, $\mathrm{t}=$ triplet, $\mathrm{q}=$ quartet, quint $=$ quintet, sext = sextet, $\mathrm{m}=$ multiplet), coupling constants $(\mathrm{Hz})$, and integration. High-resolution mass spectra (HRMS) were acquired on a Thermo Electron LTQ-Orbitrap XL Hybrid mass spectrometer on positive ESI mode. Melting points were obtained on an EZ-melting apparatus and were uncorrected. Liquid chromatography was performed using forced flow (flash chromatography) of the indicated solvent system silica gel (Fisher, 40-63 $\mu \mathrm{m}$ ) packed in glass columns. 


\section{Experimental Procedures}

\subsection{Allylation Products}

General Procedure A: Cu(OTf) 2 mediated allyl additions to imines

Aldehyde (1.0 equiv), $p$-toluenesulfonamide (1.0 equiv) and sodium benzenesulfinate salt (1.15 equiv) were combined in a $1: 1$ mixture of $\mathrm{H}_{2} \mathrm{O}$ and formic acid ( $0.66 \mathrm{M}$ in solution). After stirring at room temperature for 72 hours, the resulting white precipitate is filtered and solids washed with hexanes and dried in vacuo. Amidosulfone is isolated as a mixture of inseparable diastereomers and a white, chalky solid, used without further purification.

Amidosulfone was dissolved in $\mathrm{CH}_{2} \mathrm{Cl}_{2}(0.1 \mathrm{~mL} / \mathrm{mg}$ of amidosulfone) in a separatory funnel and shaken with aqueous saturated $\mathrm{NaHCO}_{3}(0.2 \mathrm{~mL} / \mathrm{mg})$ for one minute. The layers are separated and the organics dried with $\mathrm{Na}_{2} \mathrm{SO}_{4}$, filtered and concentrated in vacuo to afford imine as a colorless oil. Imine is used without further purification. Representative spectra of imines $\mathbf{1 2 a}, \mathbf{1 2 b}$ and 13 are reported.

A solution of imine (1.0 equiv) and allylTMS (1.1 equiv) in dry $\mathrm{CH}_{2} \mathrm{Cl}_{2}(0.1 \mathrm{M})$ was cooled to $-20{ }^{\circ} \mathrm{C}$ and then added to a flame-dried vial with pre-weighted solid $\mathrm{Cu}(\mathrm{OTf})_{2}$ (1.1 equiv). The mixture was stirred at $-20{ }^{\circ} \mathrm{C}$ overnight $(16-20 \mathrm{~h})$. The mixture was warmed to room temperature, then diluted with $\mathrm{CH}_{2} \mathrm{Cl}_{2}$ and washed with $\mathrm{H}_{2} \mathrm{O}$. The layers were separated, and the aqueous layer was extracted three times with $\mathrm{CH}_{2} \mathrm{Cl}_{2}$. Combined organic layers were dried over $\mathrm{Na}_{2} \mathrm{SO}_{4}$, filtered and concentrated in vacuo.

\section{General Procedure B: $\mathrm{Cu}(\mathrm{OTf})_{2}$ mediated multicomponent allylations}

A solution of aldehyde (1.0 equiv) and allyITMS (1.1 equiv) in dry $\mathrm{CH}_{2} \mathrm{Cl}_{2}(0.1 \mathrm{M})$ at room temperature was added to added to a flame-dried vial with pre-weighted solid $\mathrm{Cu}(\mathrm{OTf})_{2}$ (1.1 equiv) and $\mathrm{H}_{2} \mathrm{NTs}$ (1.0 equiv). The mixture was stirred at room temperature overnight (16-20 h). The mixture was diluted with $\mathrm{CH}_{2} \mathrm{Cl}_{2}$ and washed with $\mathrm{H}_{2} \mathrm{O}$. The layers were separated, and the aqueous layer was extracted three times with $\mathrm{CH}_{2} \mathrm{Cl}_{2}$. Combined organic layers were dried over $\mathrm{Na}_{2} \mathrm{SO}_{4}$, filtered and concentrated in vacuo.

** Inseparable mixtures of allylation product diastereomers were resolved upon hydrogenation using General Procedure $\mathbf{C}$ and major diastereomer fully characterized.

\section{General Procedure C: Hydrogenation of Allylation Products}

A solution of allylation product (1.0 equiv) in $\mathrm{MeOH}(0.25 \mathrm{M})$ was added to a vial containing $10 \% \mathrm{Pd} / \mathrm{C}(500 \mathrm{mg} / \mathrm{mmol}$ starting material) sparged with an Ar atmosphere. The mixture was then sparged with $\mathrm{H}_{2}$ and stirred overnight under an $\mathrm{H}_{2}$ atmosphere. After the mixture was stirred at room temperature overnight, the vial was evacuated and backfilled with Ar. The resulting mixture was filtered over celite, washed with $\mathrm{MeOH}$, and concentrated in vacuo. 


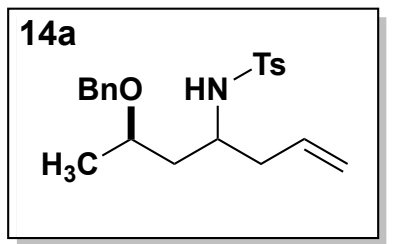

N-((6R)-6-(benzyloxy)hept-1-en-4-yl)-4-methylbenzenesulfonamide (14a)

Using general procedure $\mathbf{A}$, aldehyde $8 \mathrm{a}(1.50 \mathrm{~g}, 8.41 \mathrm{mmol})$ is combined with $p$ toluenesulfonamide $(1.44 \mathrm{~g}, 8.41 \mathrm{mmol})$ and $\mathrm{NaSO}_{2} \mathrm{Ph}(1.52 \mathrm{~g}, 9.25 \mathrm{mmol})$ in $\mathrm{H}_{2} \mathrm{O}(13 \mathrm{~mL})$ and formic acid (13 mL) to afford amidosulfone $10 \mathrm{a}(1.98 \mathrm{~g}, 50 \%)$ as a white, chalky solid. Amidosulfone 10a (500 mg) was dissolved in $\mathrm{CH}_{2} \mathrm{Cl}_{2}(50 \mathrm{~mL})$ and shaken with saturated aqueous $\mathrm{NaHCO}_{3}(25 \mathrm{~mL})$ in a separatory funnel to procure imine $12 \mathrm{a}$, used without further purification. Imine 12a (343 mg, $1.03 \mathrm{mmol}$ ), Cu(OTf) 2 (410 mg, $1.13 \mathrm{mmol}$ ), and allyITMS (179 $\mu \mathrm{L}, 1.13 \mathrm{mmol})$ were combined in $\mathrm{CH}_{2} \mathrm{Cl}_{2}(10 \mathrm{~mL})$ to afford $14 a(280 \mathrm{mg}$, $72 \%)$ as a 82:18 mixture of diastereomers. Diastereomers were purified as a mixture using flash column chromatography (15:85 to 20:80 EtOAc:hexanes) and isolated as a colorless oil.

Using general procedure $\mathbf{B}$, known aldehyde $8 \mathbf{a}(256 \mathrm{mg}, 1.496 \mathrm{mmol}), \mathrm{Cu}(\mathrm{OTf})_{2}$ (595 mg, $1.64 \mathrm{mmol}), \mathrm{H}_{2} \mathrm{NTs}(256 \mathrm{mg}, 1.496 \mathrm{mmol})$ and allylTMS (238 $\left.\mu \mathrm{L}, 1.496 \mathrm{mmol}\right)$ were combined in $\mathrm{CH}_{2} \mathrm{Cl}_{2}(15 \mathrm{~mL})$ to afford $14 \mathrm{a}$ (246 mg, 44\%) as a 78:22 mixture of diastereomers. Diastereomers were purified as a mixture using flash column chromatography (15:85 to 20:80 EtOAc:hexanes) and isolated as a colorless oil.

Major: ${ }^{1} \mathrm{H}$ NMR $\left(600 \mathrm{MHz}, \mathrm{CDCl}_{3}\right) \delta 7.71(\mathrm{dd}, \mathrm{J}=8.3,1.8 \mathrm{~Hz}, 2 \mathrm{H}), 7.43-7.21(\mathrm{~m}, 7 \mathrm{H}), 5.66$ $-5.52(\mathrm{~m}, 1 \mathrm{H}), 5.26(\mathrm{~d}, \mathrm{~J}=7.9 \mathrm{~Hz}, 1 \mathrm{H}), 4.96(\mathrm{dd}, \mathrm{J}=54.9,13.3 \mathrm{~Hz}, 2 \mathrm{H}), 4.54(\mathrm{~d}, \mathrm{~J}=11.3 \mathrm{~Hz}$, $1 \mathrm{H}), 4.32(\mathrm{~d}, \mathrm{~J}=11.1 \mathrm{~Hz}, 1 \mathrm{H}), 3.74(\mathrm{ddd}, \mathrm{J}=9.2,6.1,2.8 \mathrm{~Hz}, 1 \mathrm{H}), 3.57-3.49(\mathrm{~m}, 1 \mathrm{H}), 2.40$ (s, 3H), $2.20(\mathrm{dt}, \mathrm{J}=12.5,5.7 \mathrm{~Hz}, 1 \mathrm{H}), 2.04(\mathrm{dt}, \mathrm{J}=14.6,7.7 \mathrm{~Hz}, 1 \mathrm{H}), 1.58-1.43(\mathrm{~m}, 2 \mathrm{H})$, $1.08(\mathrm{~d}, \mathrm{~J}=6.2,1.7 \mathrm{~Hz}, 3 \mathrm{H}) .{ }^{13} \mathrm{C}\left\{{ }^{1} \mathrm{H}\right\}$ NMR $\left(151 \mathrm{MHz}, \mathrm{CDCl}_{3}\right) \delta 143.1,138.9,133.6,129.2$, 128.5, 127.9, 127.1, 117.9, 71.8, 70.5, 64.1, 50.7, 40.8, 39.8, 20.8, 19.3, 15.3.;

Minor: ${ }^{1} \mathrm{H}$ NMR $\left(600 \mathrm{MHz}, \mathrm{CDCl}_{3}\right) \delta 3.43-3.36(\mathrm{~m}, 1 \mathrm{H}), 2.42(\mathrm{~s}, 3 \mathrm{H}), 1.14(\mathrm{dd}, \mathrm{J}=6.2,1.6$ $\mathrm{Hz}, 3 \mathrm{H}) .{ }^{13} \mathrm{C}\left\{{ }^{1} \mathrm{H}\right\} \operatorname{NMR}\left(151 \mathrm{MHz}, \mathrm{CDCl}_{3}\right) \delta 143.2,138.3,133.3,127.7,127.2,119.5,73.0$, 70.0, 51.8, 41.0, 39.3, 21.5, 19.5 .

AMM (ESI-TOF) $m / z$ calculated for $\mathrm{C}_{21} \mathrm{H}_{28} \mathrm{NO}_{3} \mathrm{~S}^{+}[\mathrm{M}+\mathrm{H}]^{+} 374.1790$, found 374.1784

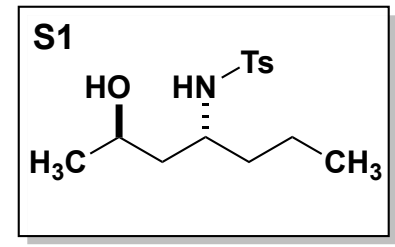

$\mathbf{N}$-((2R,4R)-2-hydroxyheptan-4-yl)-4-methylbenzenesulfonamide (S1)

Using general procedure $\mathbf{C}$, allylation product $14 \mathrm{a}(412 \mathrm{mg}, 1.10 \mathrm{mmol})$ and $10 \% \mathrm{Pd} / \mathrm{C}$ (396 mg) were combined in $\mathrm{MeOH}(4.4 \mathrm{~mL}$ ) to afford $\mathbf{S 1}$ as a mixture of diastereomers. 
The major diastereomer anti-S1 was isolated and purified using flash column chromatography (30:70 to 60:40 $\mathrm{Et}_{2} \mathrm{O}$ :hexanes) and isolated as a white solid (178 $\mathrm{mg}$, 54\%): ${ }^{1} \mathrm{H}$ NMR $\left(600 \mathrm{MHz}, \mathrm{CDCl}_{3}\right) \delta 7.75$ (d, J = 7.9 Hz, 2H), $7.28(\mathrm{~d}, \mathrm{~J}=7.9 \mathrm{~Hz}, 2 \mathrm{H}), 3.70$ (q, $\mathrm{J}=6.2 \mathrm{~Hz}, 1 \mathrm{H}), 3.28(\mathrm{p}, \mathrm{J}=6.5 \mathrm{~Hz}, 1 \mathrm{H}), 2.41(\mathrm{~s}, 3 \mathrm{H}), 1.50(\mathrm{t}, \mathrm{J}=6.5 \mathrm{~Hz}, 2 \mathrm{H}), 1.37$ (ddp, J = 26.7, 13.7, $6.4 \mathrm{~Hz}, 2 \mathrm{H}), 0.74(\mathrm{t}, \mathrm{J}=7.3 \mathrm{~Hz}, 3 \mathrm{H}) .{ }^{13} \mathrm{C}\left\{{ }^{1} \mathrm{H}\right\} \mathrm{NMR}\left(151 \mathrm{MHz}, \mathrm{CDCl}_{3}\right) \delta$ 143.4, 138.0, 129.6, 127.0, 63.8, 51.2, 43.8, 37.9, 23.4, 21.5, 18.7, 13.7.; AMM (ESI-TOF) $\mathrm{m} / \mathrm{z}$ calculated for $\mathrm{C}_{15} \mathrm{H}_{23} \mathrm{NO}_{3} \mathrm{~S}^{+}[\mathrm{M}+\mathrm{H}]^{+} 286.1477$, found 286.1471 .

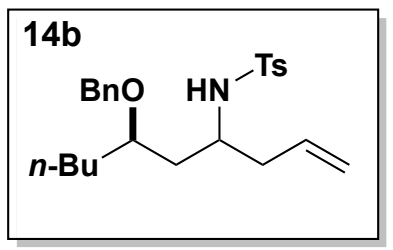

\section{$N-((6 R)-6-($ benzyloxy)dec-1-en-4-yl)-4-methylbenzenesulfonamide (14b)}

Using general procedure A, aldehyde $8 \mathbf{b}(112 \mathrm{mg}, 0.508 \mathrm{mmol})$ is combined with $p$ toluenesulfonamide $(87 \mathrm{mg}, 0.508 \mathrm{mmol})$ and $\mathrm{NaSO}_{2} \mathrm{Ph}(100 \mathrm{mg}, 0.559 \mathrm{mmol})$ in $\mathrm{H}_{2} \mathrm{O}$ $(0.77 \mathrm{~mL})$ and formic acid $(0.77 \mathrm{~mL})$ to afford amidosulfone $10 \mathrm{~b}$ (199 mg, 74\%) as a white, chalky solid. Amidosulfone $10 \mathrm{~b}$ was dissolved in $\mathrm{CH}_{2} \mathrm{Cl}_{2}(40 \mathrm{~mL})$ and shaken with saturated aqueous $\mathrm{NaHCO}_{3}(20 \mathrm{~mL})$ in a separatory funnel to procure imine $\mathbf{1 2 b}$, used without further purification. Imine 12b (125 mg, $0.334 \mathrm{mmol}), \mathrm{Cu}(\mathrm{OTf})_{2}(38 \mathrm{mg}, 0.334 \mathrm{mmol}$ ), and allylTMS (38 mg, $0.334 \mathrm{mmol}$ ) were combined in $\mathrm{CH}_{2} \mathrm{Cl}_{2}(3.3 \mathrm{~mL})$ to afford $14 \mathrm{~b}$ (102 $\mathrm{mg}$, $74 \%)$ as a 83:17 mixture of diastereomers. Diastereomers were purified as a mixture using flash column chromatography (5:95 to 20:80 EtOAc:hexanes) and isolated as a colorless oil.

Using general procedure $\mathbf{B}$, aldehyde $8 \mathbf{b}$ (134 mg, $0.610 \mathrm{mmol}), \mathrm{Cu}(\mathrm{OTf})_{2}(221 \mathrm{mg}, 0.610$ $\mathrm{mmol}$ ), $\mathrm{H}_{2} \mathrm{NTs}$ (104 mg, $0.610 \mathrm{mmol}$ ) and allylTMS (70 mg, $0.610 \mathrm{mmol}$ ) were combined in $\mathrm{CH}_{2} \mathrm{Cl}_{2}(2.4 \mathrm{~mL})$ to afford $14 \mathrm{~b}(138 \mathrm{mg}, 55 \%)$ as a $78: 22$ mixture of diastereomers. Diastereomers were purified as a mixture using flash column chromatography $15: 95$ to 20:80 EtOAc:hexanes) and isolated as a colorless oil.

Major: ${ }^{1} \mathrm{H}$ NMR $\left(400 \mathrm{MHz}, \mathrm{CDCl}_{3}\right) \delta 7.73-7.65(\mathrm{~m}, 2 \mathrm{H}), 7.42-7.21(\mathrm{~m}, 7 \mathrm{H}), 5.67-5.47$ $(\mathrm{m}, 1 \mathrm{H}), 5.30(\mathrm{~d}, J=6.3 \mathrm{~Hz}, 1 \mathrm{H}), 5.06-4.88(\mathrm{~m}, 3 \mathrm{H}), 4.55-4.44(\mathrm{~m}, 1 \mathrm{H}), 4.39-4.29(\mathrm{~m}$, $1 \mathrm{H}), 3.62-3.42(\mathrm{~m}, 2 \mathrm{H}), 2.40(\mathrm{~s}, 3 \mathrm{H}), 2.29-2.01(\mathrm{~m}, 2 \mathrm{H}), 1.73-1.36(\mathrm{~m}, 3 \mathrm{H}), 1.37-1.07$ $(\mathrm{m}, 4 \mathrm{H}), 0.92-0.82(\mathrm{~m}, 3 \mathrm{H}) ;{ }^{13} \mathrm{C}\left\{{ }^{1} \mathrm{H}\right\}$ NMR $\left(151 \mathrm{MHz}, \mathrm{CDCl}_{3}\right) \delta 143.1,138.4,138.1,133.6$, $129.5,128.5,127.9,127.7,127.2$, 118.6, 76.2, 70.9, 50.8, 39.5, 37.7, 32.6, 27.0, 22.8, 21.5, 14.0;

Minor: ${ }^{1} \mathrm{H}$ NMR $\left(400 \mathrm{MHz}, \mathrm{CDCl}_{3}\right) \delta 3.44-3.33(\mathrm{~m}, 2 \mathrm{H}) ;{ }^{13} \mathrm{C}\left\{{ }^{1} \mathrm{H}\right\}$ NMR $\left(151 \mathrm{MHz}, \mathrm{CDCl}_{3}\right) \delta$ 143.2, 137.9, 133.3, 128.4, 127.8, 127.6, 118.9, 70.1, 51.7, 39.3, 38.3, 33.0, 26.8.

AMM (ESI-TOF) $\mathrm{m} / \mathrm{z}$ calculated for $\mathrm{C}_{24} \mathrm{H}_{34} \mathrm{NO}_{3} \mathrm{~S}^{+}[\mathrm{M}+\mathrm{H}]^{+} 416.2254$, found 416.2249 


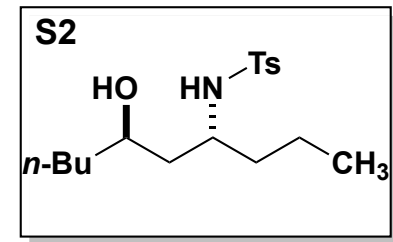

$\mathrm{N}$-((6R)-6-hydroxydecan-4-yl)-4-methylbenzenesulfonamide (S2)

Using general procedure $\mathbf{C}$, allylation product $14 \mathrm{~b}(116 \mathrm{mg}, 0.280 \mathrm{mmol})$ and $10 \% \mathrm{Pd} / \mathrm{C}$ (224 mg) were combined in $\mathrm{MeOH}(1.1 \mathrm{~mL})$ to afford $\mathbf{S 2}$ as a 70:20 mixture of diastereomers. Anti-S2 was isolated and purified using flash column chromatography (10:90 to 30:70 EtOAc:hexanes) and isolated as a colorless oil (55 mg, 60\%). Relative stereochemistry of major diastereomer was assigned based on NMR correlation to $\mathbf{S 1} .{ }^{1} \mathrm{H}$ $\operatorname{NMR}\left(400 \mathrm{MHz}, \mathrm{CDCl}_{3}\right) \delta 7.77(\mathrm{~d}, J=8.0 \mathrm{~Hz}, 2 \mathrm{H}), 7.30(\mathrm{~d}, J=8.0 \mathrm{~Hz}, 2 \mathrm{H}), 5.16-5.04(\mathrm{~m}$, $1 \mathrm{H}), 3.86(\mathrm{~s}, 1 \mathrm{H}), 3.51-3.38(\mathrm{~m}, 1 \mathrm{H}), 2.71(\mathrm{~s}, 1 \mathrm{H}), 2.43(\mathrm{~s}, 3 \mathrm{H}), 1.50-1.20(\mathrm{~m}, 10 \mathrm{H}), 1.09$ (qt, $J=14.2,6.4 \mathrm{~Hz}, 2 \mathrm{H}), 0.93-0.80(\mathrm{~m}, 3 \mathrm{H}), 0.76-0.67(\mathrm{~m}, 3 \mathrm{H}) ;{ }^{13} \mathrm{C}\left\{{ }^{1} \mathrm{H}\right\} \mathrm{NMR}(151 \mathrm{MHz}$, $\left.\mathrm{CDCl}_{3}\right) \delta 143.3,138.1,129.6,127.1,67.7,51.2,41.9,37.9,37.1,27.9,22.7,21.5,18.8$, 14.1, 13.8; AMM (ESI-TOF) $\mathrm{m} / z$ calculated for $\mathrm{C}_{17} \mathrm{H}_{30} \mathrm{NO}_{3} \mathrm{~S}^{+}[\mathrm{M}+\mathrm{H}]^{+} 328.1941$, found 328.1939 .

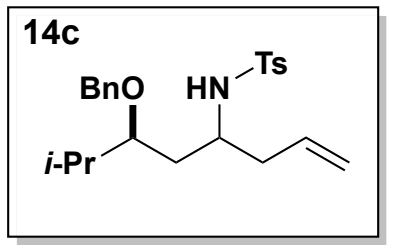

\section{$N$-((6S)-6-(benzyloxy)-7-methyloct-1-en-4-yl)-4-methylbenzenesulfonamide (14c)}

Using modified general procedure $\mathbf{A}$, aldehyde $8 \mathrm{c}(140 \mathrm{mg}, 0.678 \mathrm{mmol})$ is combined with p-toluenesulfonamide (116 mg, $0.678 \mathrm{mmol}$ ) and $\mathrm{NaSO}_{2} \mathrm{Tol}(113 \mathrm{mg}, 0.746 \mathrm{mmol})$ in $\mathrm{H}_{2} \mathrm{O}$ $(1.03 \mathrm{~mL})$ and formic acid $(1.03 \mathrm{~mL})$ to afford amidosulfone $10 \mathrm{c}$ (199 mg, 74\%) as a white, chalky solid. Amidosulfone $10 \mathrm{c}$ was dissolved in $\mathrm{CH}_{2} \mathrm{Cl}_{2}(40 \mathrm{~mL})$ and shaken with saturated aqueous $\mathrm{NaHCO}_{3}(20 \mathrm{~mL})$ in a separatory funnel to procure imine $12 \mathrm{c}(195 \mathrm{mg}, 56 \%)$ used without further purification. Imine 12c (111 mg, $0.309 \mathrm{mmol}), \mathrm{Cu}(\mathrm{OTf})_{2}(112 \mathrm{mg}, 0.309$ mmol), and allylTMS (35 mg, $0.309 \mathrm{mmol}$ ) were combined in $\mathrm{CH}_{2} \mathrm{Cl}_{2}(3 \mathrm{~mL}$ ) to afford $14 \mathrm{c}$ (56 mg, 4\%) as a 89:11 mixture of diastereomers. Diastereomers were purified as a mixture using flash column chromatography (5:95 to 20:80 EtOAc:hexanes) and isolated as a colorless oil.

Using general procedure B, aldehyde 8c (78 mg, $0.379 \mathrm{mmol}), \mathrm{Cu}(\mathrm{OTf})_{2}(137 \mathrm{mg}, 0.379$ $\mathrm{mmol}$ ), $\mathrm{H}_{2} \mathrm{NTs}$ ( $65 \mathrm{mg}, 0.379 \mathrm{mmol}$ ) and allylTMS (43 $\mathrm{mg}, 0.379 \mathrm{mmol}$ ) were combined in $\mathrm{CH}_{2} \mathrm{Cl}_{2}(1.5 \mathrm{~mL})$ to afford $14 \mathrm{c}(56 \mathrm{mg}, 37 \%)$ as a $81: 19$ mixture of diastereomers. Diastereomers were purified as a mixture using flash column chromatography $15: 95$ to 20:80 EtOAc:hexanes) and isolated as a colorless oil. 
Major: ${ }^{1} \mathrm{H} \mathrm{NMR}\left(400 \mathrm{MHz}, \mathrm{CDCl}_{3}\right) \delta 7.68(\mathrm{~d}, \mathrm{~J}=8.2 \mathrm{~Hz}, 2 \mathrm{H}), 7.41-7.13(\mathrm{~m}, 7 \mathrm{H}), 5.66-5.49$ $(\mathrm{m}, 1 \mathrm{H}), 5.21(\mathrm{~d}, J=8.0 \mathrm{~Hz}, 1 \mathrm{H}), 5.05-4.87(\mathrm{~m}, 2 \mathrm{H}), 4.56-4.47(\mathrm{~m}, 1 \mathrm{H}), 4.41-4.29(\mathrm{~m}$, $1 \mathrm{H}), 3.57-3.46(\mathrm{~m}, 1 \mathrm{H}), 3.46-3.35(\mathrm{~m}, 1 \mathrm{H}), 2.40(\mathrm{~s}, 3 \mathrm{H}), 2.25-2.10(\mathrm{~m}, 1 \mathrm{H}), 2.09-1.83$ $(\mathrm{m}, 2 \mathrm{H}), 1.50-1.35(\mathrm{~m}, 2 \mathrm{H}), 0.86-0.73(\mathrm{~m}, 6 \mathrm{H}) ;{ }^{13} \mathrm{C}\left\{{ }^{1} \mathrm{H}\right\} \mathrm{NMR}\left(151 \mathrm{MHz}, \mathrm{CDCl}_{3}\right) \delta 143.1$, $138.6,138.2,133.5,129.5,128.4,127.9,127.2,118.6,80.7,71.3,51.0,39.5,33.1,29.2$, 21.5, 18.6, 16.2;

Minor: ${ }^{1} \mathrm{H}$ NMR $\left(400 \mathrm{MHz}, \mathrm{CDCl}_{3}\right) \delta 3.19(\mathrm{q}, J=5.4 \mathrm{~Hz}, 1 \mathrm{H}), 1.53(\mathrm{t}, J=6.5 \mathrm{~Hz}, 2 \mathrm{H}) ;{ }^{13} \mathrm{C}\left\{{ }^{1} \mathrm{H}\right\}$ NMR $\left(151 \mathrm{MHz}, \mathrm{CDCl}_{3}\right) \delta 143.2,138.5,137.9,133.3,128.4,127.7,127.6,127.6,127.2$, $118.8,81.4,70.5,52.0,39.1,33.7,29.7,18.1,16.5$.

AMM (ESI-TOF) $\mathrm{m} / z$ calculated for $\mathrm{C}_{23} \mathrm{H}_{32} \mathrm{NO}_{3} \mathrm{~S}^{+}[\mathrm{M}+\mathrm{H}]^{+} 402.2097$, found 402.2106

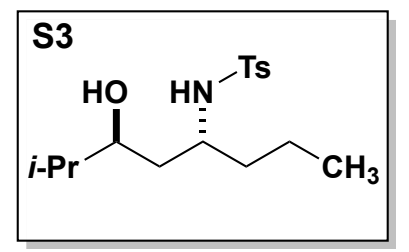

$N$-((4R,6S)-6-hydroxy-7-methyloctan-4-yl)-4-methylbenzenesulfonamide (S3)

Using general procedure $C$, allylation product $14 \mathrm{c}(56 \mathrm{mg}, 0.139 \mathrm{mmol}$ ) and $10 \% \mathrm{Pd} / \mathrm{C}$ (111 $\mathrm{mg}$ ) were combined in $\mathrm{MeOH}(0.56 \mathrm{~mL})$ to afford $\mathbf{S 3}$ as a 80:20 mixture of diastereomers. Anti-S3 was purified using flash column chromatography (10:90 to 30:70 EtOAc:hexanes) and isolated as a pale yellow oil $(9 \mathrm{mg}, 5 \%)$. Relative stereochemistry of major diastereomer was assigned based on NMR correlation to $\mathbf{S 1} .{ }^{1} \mathrm{H}$ NMR $\left(600 \mathrm{MHz}, \mathrm{cdc}_{3}\right) \delta$ $7.77(\mathrm{~d}, J=8.4 \mathrm{~Hz}, 2 \mathrm{H}), 7.30(\mathrm{~d}, J=8.0 \mathrm{~Hz}, 2 \mathrm{H}), 4.87(\mathrm{~d}, J=9.7 \mathrm{~Hz}, 1 \mathrm{H}), 3.69-3.57(\mathrm{~m}, 1 \mathrm{H})$, $3.50-3.40(\mathrm{~m}, 1 \mathrm{H}), 2.43(\mathrm{~s}, 3 \mathrm{H}), 1.61-1.51(\mathrm{~m}, 1 \mathrm{H}), 1.43(\mathrm{ddd}, J=14.1,10.8,3.2 \mathrm{~Hz}, 1 \mathrm{H})$, $1.36-1.22(\mathrm{~m}, 4 \mathrm{H}), 1.20-1.00(\mathrm{~m}, 2 \mathrm{H}), 0.86(\mathrm{dd}, J=10.5,6.8 \mathrm{~Hz}, 6 \mathrm{H}), 0.73(\mathrm{t}, J=7.3 \mathrm{~Hz}$, $3 \mathrm{H}) ;{ }^{13} \mathrm{C}\left\{{ }^{1} \mathrm{H}\right\}$ NMR $\left(151 \mathrm{MHz}, \mathrm{CDCl}_{3}\right) \delta 143.3,138.1,129.6,127.1,72.3,51.3,38.3,37.9$, $33.7,21.5,18.8,18.6,17.7,13.8$; AMM (ESI-TOF) $\mathrm{m} / z$ calculated for $\mathrm{C}_{16} \mathrm{H}_{28} \mathrm{NO}_{3} \mathrm{~S}^{+}[\mathrm{M}+\mathrm{H}]^{+}$ 314.1784 , found 314.1788

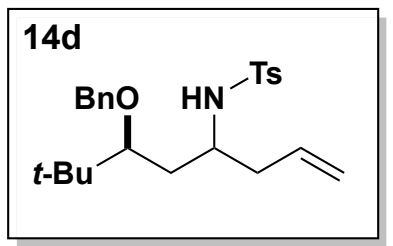

$N$-((6S)-6-(benzyloxy)-7,7-dimethyloct-1-en-4-yl)-4-methylbenzenesulfonamide (14d) Using general procedure $\mathbf{B}$, aldehyde $8 \mathrm{~d}(80 \mathrm{mg}, 0.362 \mathrm{mmol}), \mathrm{Cu}(\mathrm{OTf})_{2}(132 \mathrm{mg}, 0.362$ $\mathrm{mmol}), \mathrm{H}_{2} \mathrm{NTs}(62 \mathrm{mg}, 0.362 \mathrm{mmol}$ ) and allylTMS ( $41 \mathrm{mg}, 0.362 \mathrm{mmol}$ ) were combined in $\mathrm{CH}_{2} \mathrm{Cl}_{2}(1.45 \mathrm{~mL})$ to afford $14 \mathrm{~d}(66 \mathrm{mg}, 44 \%)$ as a $89: 11$ mixture of diastereomers. Diastereomers were purified as a mixture using flash column chromatography (5:95 to 20:80 EtOAc:hexanes) and isolated as a colorless oil. 
Major: ${ }^{1} \mathrm{H}$ NMR $\left(400 \mathrm{MHz}, \mathrm{CDCl}_{3}\right) \delta 7.75-7.64(\mathrm{~m}, 2 \mathrm{H}), 7.39-7.24(\mathrm{~m}, 7 \mathrm{H}), 5.63-5.46$ $(\mathrm{m}, 1 \mathrm{H}), 5.09-4.92(\mathrm{~m}, 1 \mathrm{H}), 4.85(\mathrm{~d}, J=17.1 \mathrm{~Hz}, 1 \mathrm{H}), 4.72-4.47(\mathrm{~m}, 3 \mathrm{H}), 3.61-3.50(\mathrm{~m}$, $1 \mathrm{H}), 3.25(\mathrm{dd}, J=8.0,3.4 \mathrm{~Hz}, 1 \mathrm{H}), 2.41(\mathrm{~s}, 3 \mathrm{H}), 2.21-2.04(\mathrm{~m}, 1 \mathrm{H}), 2.02-1.90(\mathrm{~m}, 1 \mathrm{H})$, $1.56-1.44(\mathrm{~m}, 2 \mathrm{H}), 0.90(\mathrm{~s}, 9 \mathrm{H}) ;{ }^{13} \mathrm{C}\left\{{ }^{1} \mathrm{H}\right\} \operatorname{NMR}\left(151 \mathrm{MHz}, \mathrm{CDCl}_{3}\right) \delta$ 143.4, 139.4, 138.4, $132.9,129.7,128.3,127.2,127.2,119.2,84.7,74.8,51.4,39.8,36.7,36.1,26.4,26.2,21.5$. Minor: ${ }^{1} \mathrm{H}$ NMR $\left(400 \mathrm{MHz}, \mathrm{CDCl}_{3}\right) \delta 7.20(\mathrm{~d}, J=8.0 \mathrm{~Hz}, 1 \mathrm{H}), 3.45-3.34(\mathrm{~m}, 1 \mathrm{H}), 3.00(\mathrm{dd}, J$ = 8.0, 3.3 Hz, $1 \mathrm{H}), 1.65$ (ddd, $J=14.5,7.5,3.4 \mathrm{~Hz}, 1 \mathrm{H}) ;{ }^{13} \mathrm{C}\left\{{ }^{1} \mathrm{H}\right\} \mathrm{NMR}\left(151 \mathrm{MHz}, \mathrm{CDCl}_{3}\right) \delta$ $143.2,138.9,137.9,129.6,127.4,127.3,119.3,73.8,51.9,38.6,36.3,29.7$.

AMM (ESI-TOF) $m / z$ calculated for $\mathrm{C}_{24} \mathrm{H}_{34} \mathrm{NO}_{3} \mathrm{~S}^{+}[\mathrm{M}+\mathrm{H}]^{+} 416.2254$, found 416.2244

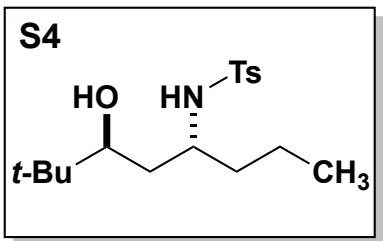

N-((4R,6S)-6-hydroxy-7,7-dimethyloctan-4-yl)-4-methylbenzenesulfonamide (S4) Using general procedure $\mathbf{C}$, allylation product 14d (35 mg, $0.084 \mathrm{mmol}$ ) and $10 \% \mathrm{Pd} / \mathrm{C}$ (67 $\mathrm{mg}$ ) were combined in $\mathrm{MeOH}(0.3 \mathrm{~mL})$ to afford $\mathbf{S 4}$ as a 90:10 mixture of diastereomers. Major diastereomer anti-S4 was purified using flash column chromatography (10:90 to 30:70 EtOAc:hexanes) and isolated as an amorphous solid (7 mg, 25\%). Relative stereochemistry of major diastereomer was assigned based on NMR correlation to $\mathbf{S 1}$.

${ }^{1} \mathrm{H}$ NMR $\left(600 \mathrm{MHz}, \mathrm{CDCl}_{3}\right) \delta 7.76(\mathrm{~d}, J=7.9 \mathrm{~Hz}, 2 \mathrm{H}), 7.30(\mathrm{~d}, J=7.9 \mathrm{~Hz}, 2 \mathrm{H}), 4.82(\mathrm{~d}, J=9.3$ $\mathrm{Hz}, 1 \mathrm{H}), 3.49-3.43(\mathrm{~m}, 2 \mathrm{H}), 2.43(\mathrm{~s}, 3 \mathrm{H}), 2.33(\mathrm{~d}, J=4.9 \mathrm{~Hz}, 1 \mathrm{H}), 1.39-1.25(\mathrm{~m}, 5 \mathrm{H}), 1.20$ $-1.04(\mathrm{~m}, 2 \mathrm{H}), 0.83(\mathrm{~s}, 10 \mathrm{H}), 0.75(\mathrm{t}, J=7.3 \mathrm{~Hz}, 3 \mathrm{H}) ;{ }^{13} \mathrm{C}\left\{{ }^{1} \mathrm{H}\right\} \mathrm{NMR}\left(151 \mathrm{MHz}, \mathrm{CDCl}_{3}\right) \delta 143.3$, 138.2 , 129.6, 127.1, 75.2, 51.5, 37.9, 35.4, 34.5, 25.6, 21.5, 18.9, 13.8; AMM (ESI-TOF) $\mathrm{m} / \mathrm{z}$ calculated for $\mathrm{C}_{17} \mathrm{H}_{30} \mathrm{NO}_{3} \mathrm{~S}^{+}[\mathrm{M}+\mathrm{H}]^{+} 328.1941$, found 328.1945

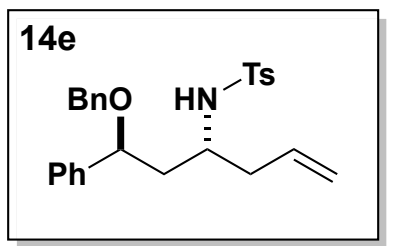

N-((1S,3R)-1-(benzyloxy)-1-phenylhex-5-en-3-yl)-4-methylbenzenesulfonamide (14e) Using modified general procedure $\mathbf{A}$, aldehyde $8 \mathrm{e}(206 \mathrm{mg}, 0.859 \mathrm{mmol})$ is combined with p-toluenesulfonamide (147 mg, $0.859 \mathrm{mmol}$ ) and $\mathrm{NaSO}_{2} \mathrm{Tol}(168 \mathrm{mg}, 0.945 \mathrm{mmol})$ in $\mathrm{H}_{2} \mathrm{O}$ $(1.30 \mathrm{~mL})$ and formic acid $(1.30 \mathrm{~mL})$ to afford amidosulfone $10 \mathrm{e}(390 \mathrm{mg}, 83 \%)$ as a white, chalky solid. Amidosulfone 10e was dissolved in $\mathrm{CH}_{2} \mathrm{Cl}_{2}(75 \mathrm{~mL})$ and shaken with saturated aqueous $\mathrm{NaHCO}_{3}(37 \mathrm{~mL})$ in a separatory funnel to procure imine $12 \mathbf{e}(182 \mathrm{mg}, 65 \%)$ used without further purification. Imine 12e (173 $\mathrm{mg}, 0.439 \mathrm{mmol}), \mathrm{Cu}(\mathrm{OTf})_{2}$ (159 $\mathrm{mg}, 0.439 \mathrm{mmol}$ ), and allylTMS (50 mg, $0.439 \mathrm{mmol}$ ) were combined in $\mathrm{CH}_{2} \mathrm{Cl}_{2}(4.4 \mathrm{~mL}$ ) to afford $14 \mathrm{e}$ as a >95:5 mixture of diastereomers. The crude material was purified using 
flash column chromatography (5:95 to 20:80 EtOAc:hexanes) and isolated as a colorless oil (108 mg, 57\%).

Using general procedure $\mathbf{B}$, a solution of aldehyde $8 \mathbf{e}(168 \mathrm{mg}, 0.7 \mathrm{mmol})$ and allylTMS (88 $\mathrm{mg}, 0.77 \mathrm{mmol}$ ) in dry $\mathrm{CH}_{2} \mathrm{Cl}_{2}(7 \mathrm{~mL}$ ) at room temperature was added to a flame-dried vial with pre-weighted solid $\mathrm{Cu}(\mathrm{OTf})_{2}$ (243 mg, $0.77 \mathrm{mmol}$ ) and $\mathrm{H}_{2} \mathrm{NTs}(120 \mathrm{mg}, 0.7 \mathrm{mmol}$ ). The mixture was stirred at room temperature overnight (16h) to afford $14 \mathrm{e}$ as a mixture of diastereomers (92:8 dr). The crude material was purified by flash chromatography (30:70 diethyl ether: hexanes) and isolated as single diastereomer anti-14e (205 mg, 76\%) as a white solid.

${ }^{1} \mathrm{H}$ NMR (400 MHz, Chloroform-d) $\delta 7.76-7.69(\mathrm{~m}, 2 \mathrm{H}), 7.42-7.25(\mathrm{~m}, 9 \mathrm{H}), 7.24-7.16$ ( $\mathrm{m}, 2 \mathrm{H}), 5.55$ (dddd, $J=16.5,10.2,8.3,6.1 \mathrm{~Hz}, 1 \mathrm{H}), 5.19(\mathrm{~d}, J=8.6 \mathrm{~Hz}, 1 \mathrm{H}), 5.05-4.97(\mathrm{~m}$, $1 \mathrm{H}), 4.91(\mathrm{dq}, J=17.0,1.6 \mathrm{~Hz}, 1 \mathrm{H}), 4.55(\mathrm{dd}, J=10.3,2.8 \mathrm{~Hz}, 1 \mathrm{H}), 4.34(\mathrm{~d}, J=11.1 \mathrm{~Hz}, 1 \mathrm{H})$, $4.19(\mathrm{~d}, J=11.0 \mathrm{~Hz}, 1 \mathrm{H}), 3.62(\mathrm{qd}, J=8.2,4.2 \mathrm{~Hz}, 1 \mathrm{H}), 2.41(\mathrm{~s}, 3 \mathrm{H}), 2.25-2.14(\mathrm{~m}, 1 \mathrm{H})$, $2.04(\mathrm{dt}, J=14.5,7.7 \mathrm{~Hz}, 1 \mathrm{H}), 1.77$ (ddd, $J=14.9,10.3,3.2 \mathrm{~Hz}, 1 \mathrm{H}), 1.62-1.51(\mathrm{~m}, 1 \mathrm{H})$.

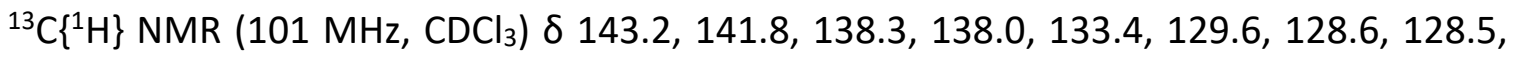
128.2, 127.8, 127.8, 127.2, 126.4, 118.8, 78.1, 77.3, 77.2, 77.0, 76.7, 70.6, 50.8, 42.4, 39.2, 21.5. AMM $m / z$ calcd $\mathrm{C}_{26} \mathrm{H}_{29} \mathrm{NO}_{3} \mathrm{~S}^{+}(\mathrm{M}+\mathrm{Na})^{+} 458.1760$, found 458.1757<smiles>[3H]NC(CC=C)C(C)C(C)OBr</smiles>

$N$-((5S,6R)-6-(benzyloxy)-5-methylhept-1-en-4-yl)-4-methylbenzenesulfonamide (14f) Using procedure $\mathbf{B}$, a solution of aldehyde $8 \mathrm{f}(168 \mathrm{mg}, 0.7 \mathrm{mmol})$ and allylTMS (109 $\mathrm{mg}$, $0.95 \mathrm{mmol})$ in dry $\mathrm{CH}_{2} \mathrm{Cl}_{2}(8.6 \mathrm{~mL} 0.1 \mathrm{M})$ at room temperature was added to a flame-dried vial with pre-weighted solid $\mathrm{Cu}(\mathrm{OTf})_{2}(313 \mathrm{mg}, 0.86 \mathrm{mmol})$ and $\mathrm{H}_{2} \mathrm{NTs}(148 \mathrm{mg}, 0.86$ $\mathrm{mmol})$. The mixture was stirred at room temperature overnight $(16 \mathrm{~h})$. The mixture was diluted with $\mathrm{CH}_{2} \mathrm{Cl}_{2}$ and washed with $\mathrm{H}_{2} \mathrm{O}$. The layers were separated, and the aqueous layer was extracted three times with $\mathrm{CH}_{2} \mathrm{Cl}_{2}$. Combined organic layers were dried over $\mathrm{Na}$ ${ }_{2} \mathrm{SO}_{4}$, filtered and concentrated in vacuo to afford $14 \mathrm{f}$ as a 51:49 mixture of diastereomers. The crude mixture was purified by flash chromatography (30:70 diethyl ether:hexanes) to afford a colorless oil (200 mg, 60\%). ${ }^{1} \mathrm{H}$ NMR (400 MHz, Chloroform-d) $\delta 7.69$ (dd, J = 8.1, $4.4 \mathrm{~Hz}, 3 \mathrm{H}), 7.43-7.23(\mathrm{~m}, 13 \mathrm{H}), 5.56(\mathrm{ddt}, \mathrm{J}=17.1,10.7,7.1 \mathrm{~Hz}, 1 \mathrm{H}), 5.35(\mathrm{~d}, \mathrm{~J}=7.9 \mathrm{~Hz}$, $1 \mathrm{H}), 5.31-5.22(\mathrm{~m}, 1 \mathrm{H}), 5.00-4.87(\mathrm{~m}, 3 \mathrm{H}), 4.61(\mathrm{dd}, \mathrm{J}=11.5,7.7 \mathrm{~Hz}, 2 \mathrm{H}), 4.46(\mathrm{t}, \mathrm{J}=3.8$ $\mathrm{Hz}, 1 \mathrm{H}), 4.40-4.29(\mathrm{~m}, 2 \mathrm{H}), 3.57-3.37(\mathrm{~m}, 3 \mathrm{H}), 2.44(\mathrm{~s}, 2 \mathrm{H}), 2.43(\mathrm{~s}, 3 \mathrm{H}), 2.25-2.03(\mathrm{~m}$, $3 \mathrm{H}), 2.02-1.89(\mathrm{~m}, 1 \mathrm{H}), 1.63(\mathrm{td}, \mathrm{J}=7.5,2.4 \mathrm{~Hz}, 1 \mathrm{H}), 1.16(\mathrm{~d}, \mathrm{~J}=6.1 \mathrm{~Hz}, 2 \mathrm{H}), 1.06(\mathrm{~d}, \mathrm{~J}=$ $6.1 \mathrm{~Hz}, 3 \mathrm{H}), 0.86$ (dd, J = 7.1, 4.6 Hz, 5H). ${ }^{13} \mathrm{C}\left\{{ }^{1} \mathrm{H}\right\} \mathrm{NMR}\left(101 \mathrm{MHz}, \mathrm{CDCl}_{3}\right) \delta$ 143.2, 143.0, $138.8,138.3,138.3,137.5,134.8,133.9,129.5,129.4,128.5,128.4,127.8,127.7,127.6$, 127.5, 127.4, 127.3, 118.8, 117.3, 77.2, 76.1, 70.6, 70.0, 55.4, 54.3, 41.7, 41.6, 36.4, 35.1, $21.5,21.5,16.8,16.2,12.7,11.5$. AMM m/z calcd $\mathrm{C}_{22} \mathrm{H}_{29} \mathrm{NO}_{3} \mathrm{~S}(\mathrm{M}+\mathrm{H})+388.1941$, found 388.1938. 
<smiles>C=CC[C@H](NC)C(C)C(C)OBr</smiles>

$N$-((5R,6R)-6-(benzyloxy)-5-methylhept-1-en-4-yl)-4-methylbenzenesulfonamide (14g) Using procedure $\mathbf{B}$, a solution of aldehyde $8 \mathrm{~g}(50 \mathrm{mg}, 0.260 \mathrm{mmol})$ and allylTMS $(0.041$ $\mathrm{mL}, 0.260 \mathrm{mmol})$ in dry $\mathrm{CH}_{2} \mathrm{Cl}_{2}(2.6 \mathrm{~mL}, 0.1 \mathrm{M})$ at room temperature was added to a flamedried vial with pre-weighted solid $\mathrm{Cu}(\mathrm{OTf})_{2}\left(94 \mathrm{mg}, 0.260 \mathrm{mmol}\right.$ ) and $\mathrm{H}_{2} \mathrm{NTs}$ (44 mg, 0.260 $\mathrm{mmol})$. The mixture was stirred at room temperature overnight $(16 \mathrm{~h})$. The mixture was diluted with $\mathrm{CH}_{2} \mathrm{Cl}_{2}$ and washed with $\mathrm{H}_{2} \mathrm{O}$. The layers were separated, and the aqueous layer was extracted three times with $\mathrm{CH}_{2} \mathrm{Cl}_{2}$. Combined organic layers were dried over $\mathrm{Na}_{2} \mathrm{SO}_{4}$, filtered and concentrated in vacuo to afford $14 \mathrm{~g}$ as a 67:33 mixture of diastereomers. The crude mixture was purified by flash chromatography and the major diastereomer, anti-14g was isolated as a colorless oil (7 mg, 7\%): ${ }^{1} \mathrm{H} \mathrm{NMR}\left(400 \mathrm{MHz}, \mathrm{CDCl}_{3}\right)$ $\delta 7.69(\mathrm{~d}, \mathrm{~J}=8.1 \mathrm{~Hz}, 2 \mathrm{H}), 7.46-7.22(\mathrm{~m}, 8 \mathrm{H}), 5.88(\mathrm{~d}, \mathrm{~J}=6.7 \mathrm{~Hz}, 1 \mathrm{H}), 5.63(\mathrm{ddt}, \mathrm{J}=17.1$, 10.1, 7.3 Hz, 1H), $5.05-4.90(\mathrm{~m}, 2 \mathrm{H}), 4.61(\mathrm{~d}, \mathrm{~J}=11.4 \mathrm{~Hz}, 1 \mathrm{H}), 4.39(\mathrm{~d}, \mathrm{~J}=11.4 \mathrm{~Hz}, 1 \mathrm{H})$, $3.86(\mathrm{qd}, \mathrm{J}=6.3,2.4 \mathrm{~Hz}, 1 \mathrm{H}$ ), 3.33 (ddd, J = 12.6, 7.2, $5.5 \mathrm{~Hz}, 1 \mathrm{H}), 2.43(\mathrm{~s}, 3 \mathrm{H}), 2.36-2.26$ (m, 1H), 2.18 (dt, J = 14.7, 7.8 Hz, 1H), 1.69 (ddt, J = 10.7, 7.2, 3.6 Hz, 1H), 1.11 (d, J = 6.3 $\mathrm{Hz}, 3 \mathrm{H}), 0.79(\mathrm{~d}, \mathrm{~J}=7.0 \mathrm{~Hz}, 3 \mathrm{H}) .{ }^{13} \mathrm{C}\left\{{ }^{1} \mathrm{H}\right\} \mathrm{NMR}\left(101 \mathrm{MHz}, \mathrm{CDCl}_{3}\right) \delta 143.0,138.7,138.3,134.2$, 129.6, 128.8, 128.2, 128.0, 127.2, 118.1, 74.3, 70.5, 57.1, 39.3, 37.9, 21.7, 16.5, 11.5. AMM $m / z$ calcd $\mathrm{C}_{26} \mathrm{H}_{29} \mathrm{NO}_{3} \mathrm{~S}^{+}(\mathrm{M}+\mathrm{Na})^{+} 388.1946$, found 388.1941.

The major product's anti configuration was determined through derivatization which is described below:

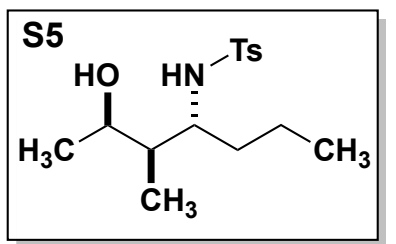

$\mathbf{N}$-((2R,3R)-2-hydroxy-3-methylheptan-4-yl)-4-methylbenzenesulfonamide (S5)

Using general procedure $\mathbf{C}$, the major product of $14 \mathrm{~g}(82 \mathrm{mg}, 0.210 \mathrm{mmol})$ and $10 \% \mathrm{Pd} / \mathrm{C}$ (100 mg) were combined in $\mathrm{MeOH}(1 \mathrm{~mL})$ to afford S5 as a single diastereomer. S5 was purified using flash column chromatography (30:70 to 60:40 $\mathrm{Et}_{2} \mathrm{O}:$ hexanes) and isolated as an amorphous solid (50 mg, 83\%): ${ }^{1} \mathrm{H}$ NMR (600 MHz, $\left.\mathrm{CDCl}_{3}\right) \delta 7.78-7.71(\mathrm{~m}, 2 \mathrm{H}), 7.29$ $(\mathrm{d}, \mathrm{J}=7.9 \mathrm{~Hz}, 2 \mathrm{H}), 5.30(\mathrm{~d}, \mathrm{~J}=8.4 \mathrm{~Hz}, 1 \mathrm{H}), 4.21(\mathrm{qd}, \mathrm{J}=6.5,2.4 \mathrm{~Hz}, 1 \mathrm{H}), 3.23(\mathrm{dq}, \mathrm{J}=8.4$, $6.3 \mathrm{~Hz}, 1 \mathrm{H}), 2.42(\mathrm{~s}, 3 \mathrm{H}), 1.50-1.33(\mathrm{~m}, 3 \mathrm{H}), 1.20-1.11(\mathrm{~m}, 4 \mathrm{H}), 1.09-0.97(\mathrm{~m}, 1 \mathrm{H}), 0.76$ $(\mathrm{t}, \mathrm{J}=7.1 \mathrm{~Hz}, 6 \mathrm{H}) .{ }^{13} \mathrm{C}\left\{{ }^{1} \mathrm{H}\right\} \mathrm{NMR}\left(151 \mathrm{MHz}, \mathrm{CDCl}_{3}\right) \delta 143.3,138.7,129.7,127.2,66.7,57.5$, 41.2, 35.6, 21.7, 21.6, 18.6, 14.2, 10.1.; AMM (ESI-TOF) $\mathrm{m} / \mathrm{z}$ calculated for $\mathrm{C}_{16} \mathrm{H}_{25} \mathrm{NO}_{3} \mathrm{~S}^{+}[\mathrm{M}$ $+\mathrm{H}]^{+} 300.1633$, found 300.1636 . 
<smiles>CCC[C@H](NC)C(C)C(C)=O</smiles>

4-methyl-N-((3R,4S)-3-methyl-2-oxoheptan-4-yl)benzenesulfonamide (S6)

S5 $(21 \mathrm{mg}, 0.0728 \mathrm{mmol}, 1.0$ equiv), derived from the major product of $\mathbf{1 4 g}$, was combined with DMP (37 mg, $0.0874 \mathrm{mmol}, 1.2$ equiv) in $\mathrm{CH}_{2} \mathrm{Cl}_{2}(0.7 \mathrm{~mL}, 0.1 \mathrm{M})$ in a flame dried $20 \mathrm{~mL}$ vial and stirred overnight $(16 \mathrm{~h})$ at room temperature to afford $\mathbf{S 6}$. The crude material was purified using flash chromatography (10:90 to 20:80 EtOAc:Hexanes) to afford $\mathbf{S 6}$ as a colorless oil (14 mg, 69\%): ${ }^{1} \mathrm{H} \mathrm{NMR}\left(600 \mathrm{MHz}, \mathrm{CDCl}_{3}\right) \delta \delta 7.75$ (d, J = $7.8 \mathrm{~Hz}$, $2 \mathrm{H}), 7.28(\mathrm{~d}, \mathrm{~J}=7.9 \mathrm{~Hz}, 2 \mathrm{H}), 5.25(\mathrm{~d}, \mathrm{~J}=9.0 \mathrm{~Hz}, 1 \mathrm{H}), 3.41(\mathrm{td}, \mathrm{J}=6.8,3.2 \mathrm{~Hz}, 1 \mathrm{H}), 2.78$ (dq, $\mathrm{J}=10.0,6.4 \mathrm{~Hz}, 1 \mathrm{H}), 2.42(\mathrm{~s}, 3 \mathrm{H}), 2.11(\mathrm{~s}, 3 \mathrm{H}), 1.35-1.22(\mathrm{~m}, 4 \mathrm{H}), 1.16(\mathrm{dq}, \mathrm{J}=14.5,7.1$ $\mathrm{Hz}, 1 \mathrm{H}), 1.06$ (d, J = 7.4 Hz, 4H), 0.74 (t, J = 7.4 Hz, 3H). ${ }^{13} \mathrm{C}\left\{{ }^{1} \mathrm{H}\right\}$ NMR (151 MHz, CDCl $)$ $\delta$ 212.2, 143.3, 138.9, 129.7, 127.1, 56.1, 49.2, 35.7, 29.9, 21.7, 19.5, 13.9, 13.7.; AMM (ESI-TOF) $\mathrm{m} / \mathrm{z}$ calculated for $\mathrm{C} 16 \mathrm{H} 25 \mathrm{NO} 3 \mathrm{NaS}+[\mathrm{M}+\mathrm{Na}]+321.1374$, found 321.1337.

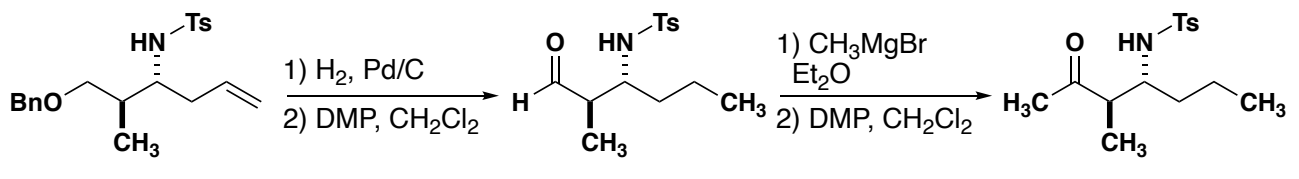

anti-17

S6

S6's relative configuration, and thus the identity of the major diastereomer of $\mathbf{1 4 g}$ was determined through transformations of anti-17 (with known relative configuration), to S6 through a procedure described below. The ${ }^{1} \mathrm{H}$ spectrum of the ketone derived from anti17 matched S6: ${ }^{1} \mathrm{H}$ NMR $\left(600 \mathrm{MHz}, \mathrm{CDCl}_{3}\right) \delta \delta 7.75(\mathrm{~d}, \mathrm{~J}=7.8 \mathrm{~Hz}, 2 \mathrm{H}), 7.28(\mathrm{~d}, \mathrm{~J}=7.9 \mathrm{~Hz}$, $2 \mathrm{H}), 5.25(\mathrm{~d}, \mathrm{~J}=9.0 \mathrm{~Hz}, 1 \mathrm{H}), 3.41(\mathrm{td}, \mathrm{J}=6.8,3.2 \mathrm{~Hz}, 1 \mathrm{H}), 2.78(\mathrm{dq}, \mathrm{J}=10.0,6.4 \mathrm{~Hz}, 1 \mathrm{H})$, $2.42(\mathrm{~s}, 3 \mathrm{H}), 2.11(\mathrm{~s}, 3 \mathrm{H}), 1.35-1.22(\mathrm{~m}, 4 \mathrm{H}), 1.16(\mathrm{dq}, \mathrm{J}=14.5,7.1 \mathrm{~Hz}, 1 \mathrm{H}), 1.06(\mathrm{~d}, \mathrm{~J}=7.4$ $\mathrm{Hz}, 4 \mathrm{H}), 0.74(\mathrm{t}, \mathrm{J}=7.4 \mathrm{~Hz}, 3 \mathrm{H})$.

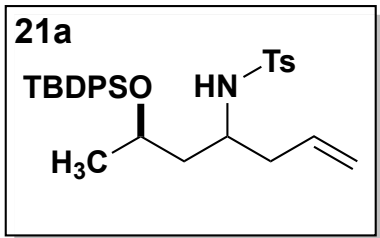

N-((6R)-6-((tert-butyldiphenylsilyl)oxy)hept-1-en-4-yl)-4-methylbenzenesulfonamide (14h)

Using general procedure B, known aldehyde $8 \mathrm{~h}$ (71 $\mathrm{mg}, 0.218 \mathrm{mmol}), \mathrm{Cu}(\mathrm{OTf})_{2}(86 \mathrm{mg}$, $0.239 \mathrm{mmol}), \mathrm{H}_{2} \mathrm{NTs}(40 \mathrm{mg}, 0.239 \mathrm{mmol})$ and allylTMS $(38 \mu \mathrm{L}, 0.239 \mathrm{mmol}$ ) were combined in $\mathrm{CH}_{2} \mathrm{Cl}_{2}(2.1 \mathrm{~mL})$ to afford $14 \mathrm{~h}$ as a $54: 46$ mixture of diastereomers. Diastereomers were purified and isolated as a mixture $(79 \mathrm{mg}, 70 \%)$ using flash column chromatography (20:80 EtOAc:hexanes) as a colorless oil. 
Mixture: ${ }^{1} \mathrm{H}$ NMR $(400 \mathrm{MHz}, \mathrm{CDCl} 3) \delta 7.85-7.59(\mathrm{~m}, 6 \mathrm{H}), 7.53-7.36(\mathrm{~m}, 6 \mathrm{H}), 7.32(\mathrm{~d}, \mathrm{~J}=$ $8.0 \mathrm{~Hz}, 1 \mathrm{H}), 7.21(\mathrm{~d}, \mathrm{~J}=8.0 \mathrm{~Hz}, 1 \mathrm{H}), 5.59(\mathrm{dd}, \mathrm{J}=6.1,2.9 \mathrm{~Hz}, 1 \mathrm{H}), 5.57-5.39(\mathrm{~m}, 1 \mathrm{H}), 5.08$ - $4.86(\mathrm{~m}, 2 \mathrm{H}), 2.32-1.95(\mathrm{~m}, 2 \mathrm{H}), 1.66-1.36(\mathrm{~m}, 2 \mathrm{H}) .{ }^{13} \mathrm{C}\left\{{ }^{1} \mathrm{H}\right\} \mathrm{NMR}\left(151 \mathrm{MHz}, \mathrm{CDCl}_{3}\right) \delta$ 143.1, 138.2, 138.1, 134.2, 129.5, 128.4, 127.8, 127.7, 127.0, 118.0, 73.9, 70.8, 57.6, 36.7, 21.5, 15.9; AMM (ESI-TOF) $m / z$ calculated for $\mathrm{C}_{30} \mathrm{H}_{3} \mathrm{NO}_{3} \mathrm{SSi}^{+}[\mathrm{M}+\mathrm{H}]^{+} 522.2498$, found 522.2497

Major: ${ }^{1} \mathrm{H}$ NMR $\left(400 \mathrm{MHz}, \mathrm{CDCl}_{3}\right) \delta 3.66(\mathrm{~h}, \mathrm{~J}=6.1 \mathrm{~Hz}, 1 \mathrm{H}), 3.29(\mathrm{dtt}, \mathrm{J}=11.5,8.1,4.0 \mathrm{~Hz}$, $1 \mathrm{H}), 2.42(\mathrm{~s}, 3 \mathrm{H}), 1.04(\mathrm{~s}, 9 \mathrm{H}), 0.91(\mathrm{~d}, \mathrm{~J}=6.0 \mathrm{~Hz}, 3 \mathrm{H})$.

Minor: ${ }^{1} \mathrm{H} \delta 3.98(\mathrm{td}, J=6.2,4.5 \mathrm{~Hz}, 1 \mathrm{H}), 3.44(\mathrm{td}, J=7.7,4.6 \mathrm{~Hz}, 1 \mathrm{H}), 2.46(\mathrm{~s}, 3 \mathrm{H}), 1.09(\mathrm{~s}$, $J=1.6 \mathrm{~Hz}, 9 \mathrm{H}), 0.83(\mathrm{~d}, J=6.3 \mathrm{~Hz}, 3 \mathrm{H})$.

Upon hydrogenation using General Procedure C, $\mathbf{1 4 h}$ was converted to fully characterized S1 in 19\% yield.

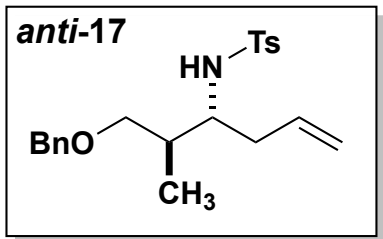

$N-((2 R, 3 R)-1-($ benzyloxy)-2-methylhex-5-en-3-yl)-4-methylbenzenesulfonamide (anti17)

Using general procedure A, aldehyde $9(890 \mathrm{mg}, 4.99 \mathrm{mmol})$ is combined with $p$ toluenesulfonamide (854 mg, $4.99 \mathrm{mmol}$ ) and $\mathrm{NaSO}_{2} \mathrm{Ph}\left(942 \mathrm{mg}, 5.74 \mathrm{mmol}\right.$ ) in $\mathrm{H}_{2} \mathrm{O}(7.5$ $\mathrm{mL}$ ) and formic acid (7.5 mL) to afford amidosulfone 11 (967 mg, 41\%) as a white, chalky solid. Amidosulfone 11 (200 mg) was dissolved in $\mathrm{CH}_{2} \mathrm{Cl}_{2}(40 \mathrm{~mL})$ and shaken with saturated aqueous $\mathrm{NaHCO}_{3}(20 \mathrm{~mL})$ in a separatory funnel to procure imine 13 , used without further purification. Imine $13\left(137 \mathrm{mg}, 0.416 \mathrm{mmol}^{2}, \mathrm{BF}_{3} \bullet \mathrm{OEt}_{2}(0.077 \mathrm{~mL}, 0.624\right.$ mmol), and allylBF $3 \mathrm{~K}(92 \mathrm{mg}, 0.624 \mathrm{mmol}), 18-$ crown-6 (165 mg, $0.624 \mathrm{mmol}$ ) were combined in $\mathrm{CH}_{2} \mathrm{Cl}_{2}(4.1 \mathrm{~mL})$ at $-20{ }^{\circ} \mathrm{C}$ for $24 \mathrm{~h}$ to afford 17 as a $52: 48$ mixture of diastereomers. Diastereomers were purified as a mixture using flash column chromatography (30:70 to 40:60 $\mathrm{Et}_{2} \mathrm{O}$ :hexanes) in 53\% yield (143 mg). Anti-17 was separated and characterized as a white solid.

Anti-17: ${ }^{1} \mathrm{H}$ NMR (400 MHz, $\left.\mathrm{CDCl}_{3}\right) \delta 7.72(\mathrm{~d}, \mathrm{~J}=8.1 \mathrm{~Hz}, 2 \mathrm{H}), 7.38-7.26(\mathrm{~m}, 7 \mathrm{H}), 5.56-$ $5.41(\mathrm{~m}, 1 \mathrm{H}), 4.96-4.84(\mathrm{~m}, 2 \mathrm{H}), 4.71(\mathrm{~d}, \mathrm{~J}=8.3 \mathrm{~Hz}, 1 \mathrm{H}), 4.56(\mathrm{~d}, \mathrm{~J}=11.6 \mathrm{~Hz}, 1 \mathrm{H}), 4.33(\mathrm{~d}$, $\mathrm{J}=11.6 \mathrm{~Hz}, 1 \mathrm{H}), 3.62(\mathrm{qd}, \mathrm{J}=6.2,2.5 \mathrm{~Hz}, 1 \mathrm{H}), 3.28-3.17(\mathrm{~m}, 1 \mathrm{H}), 2.41(\mathrm{~s}, 3 \mathrm{H}), 2.40-2.30$ $(\mathrm{m}, 1 \mathrm{H}), 2.16-2.06(\mathrm{~m}, 1 \mathrm{H}), 1.07(\mathrm{~d}, \mathrm{~J}=6.2 \mathrm{~Hz}, 3 \mathrm{H}) ;{ }^{13} \mathrm{C}\left\{{ }^{1} \mathrm{H}\right\} \mathrm{NMR}\left(151 \mathrm{MHz}, \mathrm{CDCl}_{3}\right) \delta 142.4$, 138.7, 137.1, 133.9, 129.4, 128.4, 127.7, 127.6, 127.1, 118.2, 73.2, 72.1, 56.5, 38.4, 36.1, 19.9, 14.9. Minor: AMM (ESI-TOF) $m / z$ calculated for $\mathrm{NaC}_{21} \mathrm{H}_{27} \mathrm{NO}_{3} \mathrm{~S}^{+}[\mathrm{M}+\mathrm{Na}]^{+} 374.1790$, found 374.1784 . 


\subsection{Aldehyde Synthesis}

General Procedure D: Reduction of Beta-Keto Ester and Benzylidene Acetal Formation Beta-keto ester (1.0 equiv) was added dropwise to a flame-dried round bottom flask containing a suspension of $\mathrm{LiAlH}_{4}$ (3.0 equiv) in dry $\mathrm{Et}_{2} \mathrm{O}(0.3 \mathrm{M})$. The mixture was then allowed to stir at room temperature overnight (12-20 h). Then the mixture was cooled to $0{ }^{\circ} \mathrm{C}$ and quenched using the Fieser workup: $\mathrm{H}_{2} \mathrm{O}\left(1 \mathrm{~mL} / \mathrm{g}\right.$ of $\left.\mathrm{LiAlH}_{4}\right)$ was slowly added; the reaction was allowed to stir for $15 \mathrm{~min}$ at $0{ }^{\circ} \mathrm{C} .10 \% \mathrm{NaOH}\left(1 \mathrm{~mL} / \mathrm{g}\right.$ of $\left.\mathrm{LiAlH}_{4}\right)$ was slowly added; the reaction was allowed to stir for another $15 \mathrm{~min}$ at $0{ }^{\circ} \mathrm{C}$. Finally, $\mathrm{H}_{2} \mathrm{O}(3 \mathrm{~mL} / \mathrm{g}$ of $\mathrm{LiAlH}_{4}$ ) was added; the reaction was allowed to stir for $30 \mathrm{~min}$ at room temperature. After $\mathrm{Na}_{2} \mathrm{SO}_{4}$ was added, the mixture was filtered over celite, washed with $\mathrm{Et}_{2} \mathrm{O}$, and concentrated in vacuo to afford a colorless oil. The resulting crude oil was used without further purification.

The crude diol (1.0 equiv) was dissolved in $\mathrm{CH}_{2} \mathrm{Cl}_{2}(1.0 \mathrm{M})$ in a round bottom flask. To the solution was added benzaldehyde (1.2 equiv), $\mathrm{p}-\mathrm{TsOH}$ ( 0.1 equiv), and $\mathrm{MgSO}_{4}$ (2.0 equiv). The mixture was then allowed to stir at room temperature overnight (16-22 h). The mixture was then filtered over celite and washed with $\mathrm{CH}_{2} \mathrm{Cl}_{2}$. The resulting filtrate was washed with $\mathrm{NaHCO}_{3}, \mathrm{H}_{2} \mathrm{O}$, and brine. The organic layer was then dried with $\mathrm{Na}_{2} \mathrm{SO}_{4}$, filtered, and concentrated in vacuo, affording a pale yellow oil.

\section{General Procedure E: Cu(OTf) 2 Acetal Opening}

Acetal (1.0 equiv) and $\mathrm{BH}_{3} \bullet \mathrm{THF}(1.0 \mathrm{M}, 5.0$ equiv) were added to a flame-dried round bottom flask. The resulting mixture was stirred at room temperature for 10 minutes before adding $\mathrm{Cu}(\mathrm{OTf})_{2}$ ( 0.1 equiv). The mixture was allowed to stir at room temperature until complete consumption of starting material occurred (1-3 h), as indicated by TLC. The mixture was then cooled to $0{ }^{\circ} \mathrm{C}$. $\mathrm{NEt}_{3}$ (1.0 equiv) was slowly added, followed by $\mathrm{MeOH}$ (44.5 equiv). The resulting mixture was concentrated in vacuo, affording a colorless oil.

\section{General Procedure F: Dess-Martin Oxidation of Benzylated Alcohol}

Dess Martin periodinane (1.2 equiv) was added to a solution of benzylated alcohol (1.0 equiv) in $\mathrm{CH}_{2} \mathrm{Cl}_{2}(0.1 \mathrm{M})$. The resulting mixture was allowed to stir at room temperature for $1 \mathrm{~h}$. The reaction was quenched with sat. $\mathrm{Na}_{2} \mathrm{SO}_{3}$ and $\mathrm{Et}_{2} \mathrm{O}$, then allowed to stir for an additional 10 minutes or until all of the solid dissolved. The organic layer was washed with sat. $\mathrm{Na}_{2} \mathrm{SO}_{3}$, then sat. $\mathrm{NaHCO}_{3}$, then $\mathrm{H}_{2} \mathrm{O}$, and brine. The organic layer was then dried with $\mathrm{Na}_{2} \mathrm{SO}_{4}$, filtered, and concentrated in vacuo, affording a colorless oil.

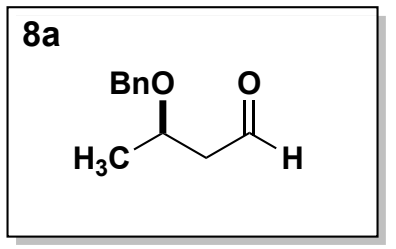

(R)-3-(benzyloxy)butanal (8a) 
Known aldehyde 8 a was prepared according to previously reported procedure. ${ }^{1}$ Spectral data matches previously reported data. ${ }^{1}{ }^{1} \mathrm{H}$ NMR $(599 \mathrm{MHz}$, Chloroform- $d) \delta 9.78(\mathrm{t}, J=$ $2.1 \mathrm{~Hz}, 1 \mathrm{H}), 7.38-7.27(\mathrm{~m}, 5 \mathrm{H}), 4.59(\mathrm{~d}, J=11.6 \mathrm{~Hz}, 1 \mathrm{H}), 4.47(\mathrm{~d}, J=11.6 \mathrm{~Hz}, 1 \mathrm{H}), 4.07$ $(p, J=6.3 \mathrm{~Hz}, 1 \mathrm{H}), 2.69(\mathrm{ddt}, J=16.4,7.5,2.0 \mathrm{~Hz}, 1 \mathrm{H}), 2.51(\mathrm{dt}, J=16.4,3.2 \mathrm{~Hz}, 1 \mathrm{H}), 1.29$ (dd, $J=6.2,1.5 \mathrm{~Hz}, 3 \mathrm{H}$ ).

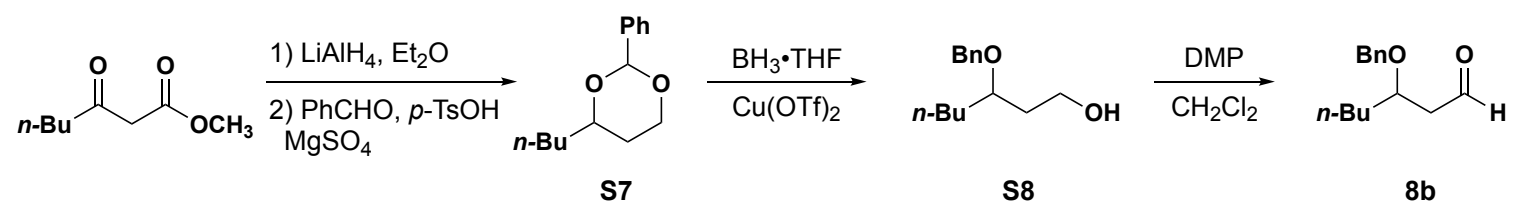

(4R)-4-butyl-2-phenyl-1,3-dioxane (S7)

Using general procedure $\mathbf{D}$, commercially available ethyl 3-oxoheptanoate $(1.5 \mathrm{~mL}, 9.54$ $\mathrm{mmol})$ and $\mathrm{LAH}(1.086 \mathrm{~g}, 28.62 \mathrm{mmol})$ were combined in $\mathrm{Et}_{2} \mathrm{O}(31.8 \mathrm{~mL})$. The resulting crude oil (1.121 g, $8.48 \mathrm{mmol})$, benzaldehyde (1.04 mL, $10.18 \mathrm{mmol}), \mathrm{p}-\mathrm{TsOH} \bullet \mathrm{H}_{2} \mathrm{O}$ (161 $\mathrm{mg}, 0.848 \mathrm{mmol})$, and $\mathrm{MgSO}_{4}\left(2.042 \mathrm{~g}, 16.96 \mathrm{mmol}\right.$ ) were combined in $\mathrm{CH}_{2} \mathrm{Cl}_{2}(8.5 \mathrm{~mL})$ to afford S7. S7 was purified using flash column chromatography (1:99 to 20:80 EtOAc:hexanes) and isolated as a pale yellow oil ( $607 \mathrm{mg}, 29 \%$ over 2 steps). ${ }^{1} \mathrm{H}$ NMR (400 $\left.\mathrm{MHz} \mathrm{CDCl}_{3}\right) \delta 7.58-7.46(\mathrm{~m}, 2 \mathrm{H}), 7.40-7.27(\mathrm{~m}, 3 \mathrm{H}), 5.50(\mathrm{~s}, 1 \mathrm{H}), 4.26(\mathrm{dd}, J=11.3,5.0$ $\mathrm{Hz}, 1 \mathrm{H}), 3.95(\mathrm{td}, J=11.9,2.6 \mathrm{~Hz}, 1 \mathrm{H}), 3.87-3.76(\mathrm{~m}, 1 \mathrm{H}), 1.88-1.62(\mathrm{~m}, 2 \mathrm{H}), 1.60-1.28$ $(\mathrm{m}, 6 \mathrm{H}), 0.91(\mathrm{t}, J=7.0 \mathrm{~Hz}, 3 \mathrm{H}) ;{ }^{13} \mathrm{C}$ NMR (151 MHz, Acetone-d) $\delta$ 201.3, 139.1, 128.1, $127.4,127.2,81.7,73.1,45.6,35.4,25.5$; AMM (ESI-TOF) $\mathrm{m} / \mathrm{z}$ calculated for $\mathrm{C}_{14} \mathrm{H}_{21} \mathrm{O}_{2}{ }^{+}$[M $+\mathrm{H}]^{+} 221.1536$, found 221.1539

\section{3-(benzyloxy)heptan-1-ol (S8)}

Using general procedure E, benzylidene acetal $\mathbf{S 7}\left(599 \mathrm{mg}, 2.71 \mathrm{mmol}\right.$ ), $\mathrm{BH}_{3} \bullet \mathrm{THF}(15.1$ $\mathrm{mL}, 13.59 \mathrm{mmol})$, and $\mathrm{Cu}(\mathrm{OTf})_{2}(98 \mathrm{mg}, 0.271 \mathrm{mmol})$, were combined to afford $\mathbf{S 8}$. S8 was purified using flash column chromatography (5:95 to 50:50 EtOAc:hexanes) and isolated as a colorless oil (396 mg, 66\%). ${ }^{1} \mathrm{H}$ NMR $\left(400 \mathrm{MHz}, \mathrm{CDCl}_{3}\right) \delta 7.37-7.24(\mathrm{~m}, 5 \mathrm{H}), 4.59(\mathrm{~d}$, $J=11.4 \mathrm{~Hz}, 1 \mathrm{H}), 4.48(\mathrm{~d}, J=11.4 \mathrm{~Hz}, 1 \mathrm{H}), 3.84-3.58(\mathrm{~m}, 3 \mathrm{H}), 2.52(\mathrm{~s}, 1 \mathrm{H}), 1.88-1.43(\mathrm{~m}$, $3 \mathrm{H}), 1.42-1.23(\mathrm{~m}, 5 \mathrm{H}), 0.93-0.89(\mathrm{~m}, 3 \mathrm{H}) ;{ }^{13} \mathrm{C} \mathrm{NMR}\left(151 \mathrm{MHz}, \mathrm{CDCl}_{3}\right) \delta$ 138.5, 128.5, $127.9,127.7,78.6,70.9,60.8,35.9,33.1,27.3,22.9,14.1$; AMM (ESI-TOF) $\mathrm{m} / \mathrm{z}$ calculated for $\mathrm{C}_{14} \mathrm{H}_{23} \mathrm{O}_{2}{ }^{+}[\mathrm{M}+\mathrm{H}]^{+} 223.1693$, found 223.1697

\section{3-(benzyloxy)heptanal (8b)}

Using general procedure $\mathbf{F}$, alcohol $\mathbf{S 8}(364 \mathrm{mg}, 1.64 \mathrm{mmol}$ ) was combined with DMP (834 $\mathrm{mg}, 1.97 \mathrm{mmol})$ in $\mathrm{CH}_{2} \mathrm{Cl}_{2}(16.4 \mathrm{~mL})$ to produce aldehyde $8 \mathrm{~b}(274 \mathrm{mg}, 76 \%)$. Compound $8 \mathrm{~b}$ was used without further purification in subsequent reactions. ${ }^{1} \mathrm{H}$ NMR of known compound $8 \mathbf{b}$ matched with previously reported data. ${ }^{2}{ }^{1} \mathrm{H} \mathrm{NMR}\left(400 \mathrm{MHz}, \mathrm{CDCl}_{3}\right) \delta 9.80$ $(\mathrm{t}, J=2.3 \mathrm{~Hz}, 1 \mathrm{H}), 7.31(\mathrm{qd}, J=8.6,7.6,4.1 \mathrm{~Hz}, 5 \mathrm{H}), 4.57(\mathrm{~d}, J=11.4 \mathrm{~Hz}, 1 \mathrm{H}), 4.52(\mathrm{~d}, J=$ $11.5 \mathrm{~Hz}, 1 \mathrm{H}), 4.01-3.89(\mathrm{~m}, 1 \mathrm{H}), 2.68$ (ddd, $J=16.3,7.2,2.6 \mathrm{~Hz}, 1 \mathrm{H}), 2.56$ (ddd, $J=16.3$, $4.8,1.9 \mathrm{~Hz}, 1 \mathrm{H}), 1.77-1.63(\mathrm{~m}, 1 \mathrm{H}), 1.63-1.49(\mathrm{~m}, 1 \mathrm{H}), 1.35(\mathrm{dtd}, J=14.7,10.2,8.1,5.6$ $\mathrm{Hz}, 4 \mathrm{H}), 0.94-0.86(\mathrm{~m}, 3 \mathrm{H})$. 


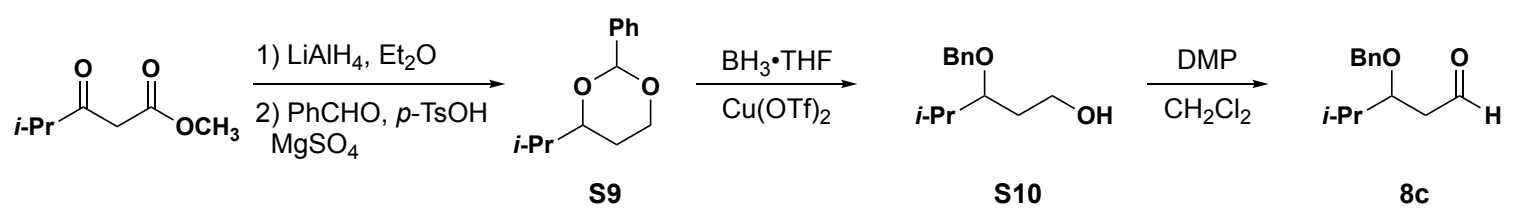

\section{4-isopropyl-2-phenyl-1,3-dioxane (S9)}

Using general procedure $\mathbf{D}$, commercially available methyl isobutyrylacetate $(1.0 \mathrm{~mL}$, $7.03 \mathrm{mmol})$ and $\mathrm{LAH}(800 \mathrm{mg}, 21.08 \mathrm{mmol})$ were combined in $\mathrm{Et}_{2} \mathrm{O}(23.4 \mathrm{~mL})$. The resulting crude oil $(629 \mathrm{~g}, 5.32 \mathrm{mmol}), \mathrm{PhCHO}(0.65 \mathrm{~mL}, 6.38 \mathrm{mmol}), \mathrm{p}-\mathrm{TsOH} \bullet \mathrm{H}_{2} \mathrm{O}(101$ $\mathrm{mg}, 0.572 \mathrm{mmol})$, and $\mathrm{MgSO}_{4}(1.28 \mathrm{~g}, 10.6 \mathrm{mmol})$ were combined in $\mathrm{CH}_{2} \mathrm{Cl}_{2}(5.32 \mathrm{~mL})$ to afford $\mathbf{5 9}$ as an 80:20 mixture of inseparable diastereomers. $\mathbf{5 9}$ was purified using flash column chromatography (0:100 to 20:80 EtOAc:hexanes) and isolated as a colorless oil (673 mg, 46\% over 2 steps). ${ }^{1} \mathrm{H}$ NMR of known compound $\mathbf{S 9}$ matched with previously reported data. ${ }^{3}{ }^{1} \mathrm{H}$ NMR $\left(400 \mathrm{MHz}, \mathrm{CDCl}_{3}\right) \delta 7.92-7.85(\mathrm{~m}, 1 \mathrm{H}), 7.58-7.46(\mathrm{~m}, 3 \mathrm{H}), 7.39$ $-7.28(\mathrm{~m}, 3 \mathrm{H}), 5.50(\mathrm{~s}, 1 \mathrm{H}), 4.29$ (ddd, $J=11.4,5.0,1.5 \mathrm{~Hz}, 1 \mathrm{H}$ ), 3.94 (ddd, $J=12.4,11.3$, $2.6 \mathrm{~Hz}, 1 \mathrm{H}$ ), 3.52 (ddd, $J=11.3,6.8,2.4 \mathrm{~Hz}, 1 \mathrm{H}), 1.93-1.68(\mathrm{~m}, 2 \mathrm{H}), 1.53(\mathrm{dtd}, J=13.2$, 2.5, $1.5 \mathrm{~Hz}, 1 \mathrm{H}), 1.02(\mathrm{~d}, J=6.7 \mathrm{~Hz}, 3 \mathrm{H}), 0.95(\mathrm{~d}, J=6.8 \mathrm{~Hz}, 3 \mathrm{H})$.

\section{3-(benzyloxy)-4-methylpentan-1-ol (S10)}

Using general procedure $\mathbf{E}$, benzylidene acetal $\mathbf{S 9}(673 \mathrm{mg}, 3.26 \mathrm{mmol}), \mathrm{BH}_{3} \bullet$ THF (18.1 $\mathrm{mL}, 18.1 \mathrm{mmol})$, and $\mathrm{Cu}(\mathrm{OTf})_{2}(118 \mathrm{mg}, 0.326 \mathrm{mmol})$, were combined to afford S10. S10 was purified using flash column chromatography (5:95 to 30:70 EtOAc:hexanes) and isolated as a colorless oil (480 mg, 71\%). ${ }^{1} \mathrm{H}$ NMR of known compound $\mathbf{S 1 0}$ matched with previously reported data. ${ }^{4} \mathrm{H}$ NMR $\left(400 \mathrm{MHz}, \mathrm{CDCl}_{3}\right) \delta 7.39-7.32(\mathrm{~m}, 6 \mathrm{H}), 4.62(\mathrm{~d}, J=$ $11.2 \mathrm{~Hz}, 1 \mathrm{H}), 4.48(\mathrm{~d}, J=11.3 \mathrm{~Hz}, 1 \mathrm{H}), 3.84-3.69(\mathrm{~m}, 2 \mathrm{H}), 3.45(\mathrm{dt}, J=11.6,5.4 \mathrm{~Hz}, 1 \mathrm{H})$, $2.33(\mathrm{~s}, 1 \mathrm{H}), 2.06(\mathrm{pd}, J=6.9,5.1 \mathrm{~Hz}, 1 \mathrm{H}), 1.73(\mathrm{q}, J=6.1 \mathrm{~Hz}, 2 \mathrm{H}), 0.94(\mathrm{~d}, J=2.7 \mathrm{~Hz}, 3 \mathrm{H})$, $0.93(\mathrm{~d}, J=2.7 \mathrm{~Hz}, 3 \mathrm{H})$.

\section{3-(benzyloxy)-4-methylpentanal (8c)}

Using general procedure $\mathbf{F}$, benzylated alcohol $\mathbf{S 1 0}(108 \mathrm{mg}, 0.521 \mathrm{mmol}$ ) and DMP (265 $\mathrm{mg}, 0.626 \mathrm{mmol}$ ) were combined in $\mathrm{CH}_{2} \mathrm{Cl}_{2}(5.2 \mathrm{~mL})$ to afford $8 \mathrm{c}(78 \mathrm{mg}, 73 \%) .8 \mathrm{c}$ was used without further purification. ${ }^{1} \mathrm{H} N M R$ of known compound $8 \mathrm{c}$ matched with previously reported data. ${ }^{1} \mathrm{H}$ NMR $\left(400 \mathrm{MHz}, \mathrm{CDCl}_{3}\right) \delta 9.84$ (dd, $\left.J=2.7,1.8 \mathrm{~Hz}, 1 \mathrm{H}\right), 7.41-7.29(\mathrm{~m}$, $5 \mathrm{H}), 4.60(\mathrm{~d}, J=11.4 \mathrm{~Hz}, 1 \mathrm{H}), 4.54(\mathrm{~d}, J=11.5 \mathrm{~Hz}, 1 \mathrm{H}), 3.82(\mathrm{ddd}, J=8.5,5.1,3.8 \mathrm{~Hz}, 1 \mathrm{H})$, 2.67 (ddd, $J=16.4,8.2,2.7 \mathrm{~Hz}, 1 \mathrm{H}$ ), 2.52 (ddd, $J=16.4,3.9,1.8 \mathrm{~Hz}, 1 \mathrm{H}$ ), 2.06 (dtd, $J=13.8$, 6.9, $5.1 \mathrm{~Hz}, 1 \mathrm{H}), 0.98(\mathrm{~d}, J=2.1 \mathrm{~Hz}, 3 \mathrm{H}), 0.96(\mathrm{~d}, J=2.1 \mathrm{~Hz}, 3 \mathrm{H})$.

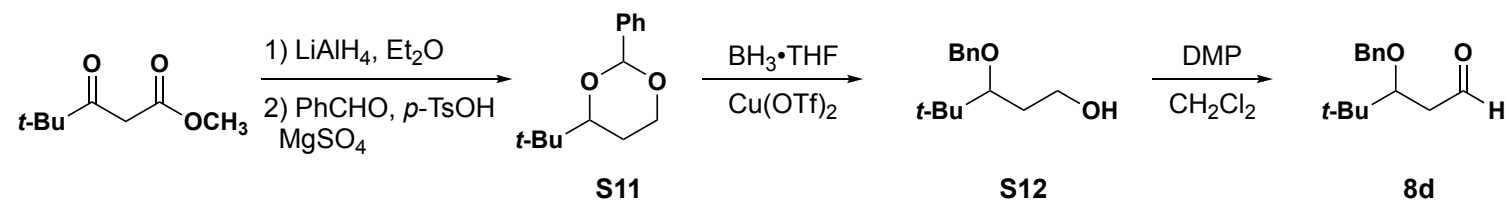

\section{4-(tert-butyl)-2-phenyl-1,3-dioxane (S11)}


Using general procedure $\mathbf{D}$, methyl 3-hydroxy-4,4-dimethylpentanoate $(1.0 \mathrm{~mL}, 6.26$ $\mathrm{mmol})$ and $\mathrm{LAH}(712 \mathrm{mg}, 18.77 \mathrm{mmol})$ were combined in $\mathrm{Et}_{2} \mathrm{O}(20.9 \mathrm{~mL})$. The resulting crude oil (629 mg, $4.76 \mathrm{mmol}), \mathrm{PhCHO}(0.58 \mathrm{~mL}, 5.71 \mathrm{mmol}), \mathrm{p}-\mathrm{TsOH} \bullet \mathrm{H}_{2} \mathrm{O}(91 \mathrm{mg}, 0.476$ mmol), and $\mathrm{MgSO}_{4}(1.146 \mathrm{~g}, 9.52 \mathrm{mmol})$ were combined in $\mathrm{CH}_{2} \mathrm{Cl}_{2}(4.8 \mathrm{~mL})$ to afford S11. S11 was purified using flash column chromatography (1:99 to 10:90 EtOAc:hexanes) and isolated as a colorless oil (446 mg, 32\% over 2 steps). ${ }^{1} \mathrm{H} \mathrm{NMR}\left(400 \mathrm{MHz}, \mathrm{CDCl}_{3}\right) \delta 7.53-$ $7.46(\mathrm{~m}, 2 \mathrm{H}), 7.41-7.27(\mathrm{~m}, 3 \mathrm{H}), 5.50(\mathrm{~s}, 1 \mathrm{H}), 4.30(\mathrm{dd}, J=11.3,5.1 \mathrm{~Hz}, 1 \mathrm{H}), 3.94(\mathrm{td}, J=$ 11.8, $2.6 \mathrm{~Hz}, 1 \mathrm{H}), 3.46(\mathrm{dd}, J=11.5,2.2 \mathrm{~Hz}, 1 \mathrm{H}), 1.93-1.78(\mathrm{~m}, 1 \mathrm{H}), 1.51-1.41(\mathrm{~m}, 1 \mathrm{H})$, 0.97 (s, 9H); ${ }^{13} \mathrm{C}$ NMR (151 MHz, $\left.\mathrm{CDCl}_{3}\right) \delta 139.3,128.5,128.1,126.0,101.0,84.8,67.3$, 34.1, 25.7, 25.6; AMM (ESI-TOF) $\mathrm{m} / \mathrm{z}$ calculated for $\mathrm{C}_{14} \mathrm{H}_{21} \mathrm{O}_{2}{ }^{+}[\mathrm{M}+\mathrm{H}]^{+} 221.1536$, found 221.1540

\section{3-(benzyloxy)-4,4-dimethylpentan-1-ol (S12)}

Using general procedure $\mathbf{E}$, benzylidene acetal $\mathbf{S 1 1}$ (297 mg, $1.35 \mathrm{mmol}$ ), $\mathrm{BH}_{3} \bullet$ THF (7.49 $\mathrm{mL}, 6.74 \mathrm{mmol}$ ), and $\mathrm{Cu}(\mathrm{OTf})_{2}(49 \mathrm{mg}, 0.135 \mathrm{mmol})$, were combined to afford S12. S12 was purified using flash column chromatography (5:95 to 50:50 EtOAc:hexanes) and isolated as a colorless oil (217 mg, 72\%). ${ }^{1} \mathrm{H}$ NMR (400 MHz, $\left.\mathrm{CDCl}_{3}\right) \delta 7.40-7.22(\mathrm{~m}, 5 \mathrm{H})$, $4.70-4.58(\mathrm{~m}, 2 \mathrm{H}), 3.83-3.67(\mathrm{~m}, 2 \mathrm{H}), 3.26(\mathrm{dd}, J=9.8,3.0 \mathrm{~Hz}, 1 \mathrm{H}), 1.93(\mathrm{~s}, 1 \mathrm{H}), 1.87-$ $1.63(\mathrm{~m}, 2 \mathrm{H}), 0.96(\mathrm{~s}, 9 \mathrm{H}) ;{ }^{13} \mathrm{C}$ NMR $\left(151 \mathrm{MHz}, \mathrm{CDCl}_{3}\right) \delta$ 138.8, 128.4, 127.6, 127.5, 85.9, 75.1, 61.0, 36.1, 33.6, 26.4; AMM (ESI-TOF) $\mathrm{m} / \mathrm{z}$ calculated for $\mathrm{C}_{14} \mathrm{H}_{23} \mathrm{O}_{2}{ }^{+}[\mathrm{M}+\mathrm{H}]^{+}$ 223.1693 , found 223.1694

\section{3-(benzyloxy)-4,4-dimethylpentanal (8d)}

Using general procedure $\mathbf{F}$, benzylated alcohol $\mathbf{S 1 2}(172 \mathrm{mg}, 0.773 \mathrm{mmol}$ ) and DMP (393 $\mathrm{mg}, 0.927 \mathrm{mmol}$ ) were combined in $\mathrm{CH}_{2} \mathrm{Cl}_{2}(7.7 \mathrm{~mL})$ to afford $8 \mathrm{~d}$. $8 \mathbf{d}$ was purified using flash column chromatography (5:95 to 20:80 EtOAc:hexanes) and isolated as a colorless oil (153 mg, 90\%). ${ }^{1} \mathrm{H} \mathrm{NMR}\left(400 \mathrm{MHz}, \mathrm{CDCl}_{3}\right) \delta 9.86(\mathrm{t}, J=2.0 \mathrm{~Hz}, 1 \mathrm{H}), 7.38-7.22(\mathrm{~m}, 5 \mathrm{H})$, $4.61(\mathrm{~d}, J=11.3 \mathrm{~Hz}, 1 \mathrm{H}), 4.54(\mathrm{~d}, J=11.3 \mathrm{~Hz}, 1 \mathrm{H}), 3.66(\mathrm{dd}, J=6.8,4.5 \mathrm{~Hz}, 1 \mathrm{H}), 2.74-2.58$ (m, 2H), $0.96(\mathrm{~s}, 9 \mathrm{H}) ;{ }^{13} \mathrm{C}$ NMR (151 MHz, Acetone) $\delta 139.8,128.2,127.8,126.2,100.8$, $76.8,66.6,35.7,31.4,27.1,22.4,13.4 ;$ AMM (ESI-TOF) $m / z$ calculated for $\mathrm{C}_{14} \mathrm{H}_{21} \mathrm{O}_{2}{ }^{+}[\mathrm{M}+$ $\mathrm{H}]^{+} 221.1536$, found 221.1540

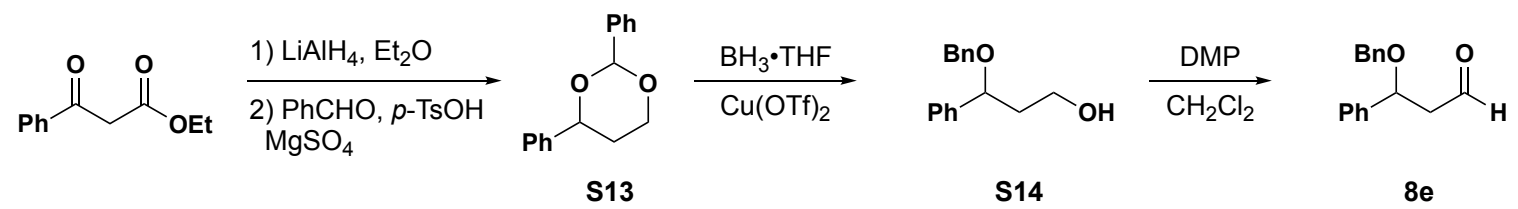

\section{2,4-diphenyl-1,3-dioxane (S13)}

Using modified general procedure $\mathbf{D}$, ethyl benzoylacetate $(2.0 \mathrm{~g}, 10.4 \mathrm{mmol})$ and LAH $(1.18 \mathrm{~g}, 31.0 \mathrm{mmol})$ were combined in $\mathrm{Et}_{2} \mathrm{O}(34 \mathrm{~mL})$. The resulting crude oil $(1.58 \mathrm{~g}, 10.4$ $\mathrm{mmol})$, benzaldehyde dimethyl acetal $(1.74 \mathrm{~g}, 11.4 \mathrm{mmol}), p$-toluenesulfonic acid (177 $\mathrm{mg}, 1.04 \mathrm{mmol}$ ) were combined in $\mathrm{CH}_{2} \mathrm{Cl}_{2}(34 \mathrm{~mL})$ to afford S13. S13 was purified using flash column chromatography (5:95 $\mathrm{Et}_{2} \mathrm{O}$ :hexanes) and isolated as a colorless oil (1.67 $\mathrm{g}$, $67 \%$ over 2 steps). ${ }^{1} \mathrm{H} \mathrm{NMR}^{5}$ is consistent with literature: ${ }^{1} \mathrm{H} \mathrm{NMR}\left(400 \mathrm{MHz}, \mathrm{CDCl}_{3}\right) \delta 7.62$ $-7.57(\mathrm{~m}, 2 \mathrm{H}), 7.48-7.44(\mathrm{~m}, 2 \mathrm{H}), 7.43-7.35(\mathrm{~m}, 6 \mathrm{H}), 5.75(\mathrm{~s}, 1 \mathrm{H}), 4.95(\mathrm{dd}, \mathrm{J}=11.4,2.6$ 
$\mathrm{Hz}, 1 \mathrm{H}), 4.40$ (ddd, $J=11.5,4.9,1.4 \mathrm{~Hz}, 1 \mathrm{H}), 4.21-4.16(\mathrm{~m}, 1 \mathrm{H}), 2.21-2.09(\mathrm{~m}, 1 \mathrm{H}), 1.83$ (dtd, $J=13.4,2.5,1.4 \mathrm{~Hz}, 1 \mathrm{H}$ ).

\section{3-(benzyloxy)-3-phenylpropan-1-ol (S14)}

Using general procedure $\mathbf{E}$, benzylidene acetal $\mathbf{S 1 3}$ (500 mg, $2.08 \mathrm{mmol}$ ), $\mathrm{BH}_{3} \bullet$ THF $(10.0$ $\mathrm{mL}, 10.0 \mathrm{mmol}$ ), and $\mathrm{Cu}(\mathrm{OTf})_{2}(38 \mathrm{mg}, 0.100 \mathrm{mmol})$, were combined to afford S14. S14 was purified using flash column chromatography (30:70 EtOAc:hexanes) and isolated as a colorless oil (325 mg, 80\%). ${ }^{1} \mathrm{H} \mathrm{NMR}^{6}$ is consistent with literature: ${ }^{1} \mathrm{H}$ NMR $(400 \mathrm{MHz}$, $\left.\mathrm{CDCl}_{3}\right) \delta 7.38-7.25(\mathrm{~m}, 10 \mathrm{H}, \mathrm{Ar}), 4.58(\mathrm{dd}, J=4.0,9.1 \mathrm{~Hz}, 1 \mathrm{H}), 4.48(\mathrm{~d}, J=11.6 \mathrm{~Hz}, 1 \mathrm{H})$, $4.27(\mathrm{~d}, J=11.6 \mathrm{~Hz}, 1 \mathrm{H}), 3.78-3.74(\mathrm{~m}, 2 \mathrm{H}), 2.09-2.06(\mathrm{~m}, 1 \mathrm{H}), 1.91-1.87(\mathrm{~m}, 1 \mathrm{H})$

\section{3-(benzyloxy)-3-phenylpropanal (8e)}

Using general procedure $\mathbf{F}$, alcohol $\mathbf{S 1 4}(170 \mathrm{mg}, 0.700 \mathrm{mmol}$ ) and DMP (328 $\mathrm{mg}, 0.770$ mmol) were combined in $\mathrm{CH}_{2} \mathrm{Cl}_{2}(7.0 \mathrm{~mL})$ to afford 8 e. $8 \mathrm{e}$ was used in subsequent reactions with no further purification. ${ }^{1} \mathrm{H} \mathrm{NMR}^{7}$ is consistent with literature: ${ }^{1} \mathrm{H}$ NMR (400 $\left.\mathrm{MHz} \mathrm{CDCl}_{3}\right) \delta 9.81(\mathrm{dd}, J=2.6,1.6 \mathrm{~Hz}, 1 \mathrm{H}), 7.44-7.30(\mathrm{~m}, 12 \mathrm{H}), 4.50(\mathrm{~d}, J=11.6 \mathrm{~Hz}, 1 \mathrm{H})$, $4.33(\mathrm{~d}, J=11.6 \mathrm{~Hz}, 1 \mathrm{H}$ ), 3.00 (ddd, $J=16.4,9.2,2.6 \mathrm{~Hz}, 1 \mathrm{H}$ ), 2.68 (ddd, $J=16.5,4.2,1.7$ $\mathrm{Hz}, 1 \mathrm{H})$.

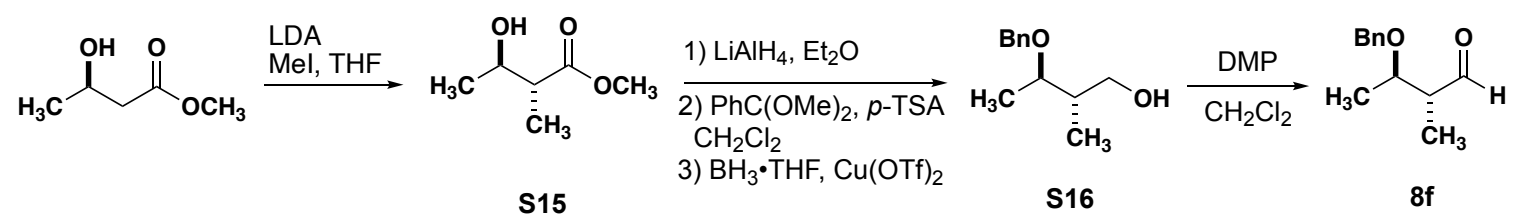

\section{Methyl (2R,3R)-3-hydroxy-2-methylbutanoate (S15)>}

Using previously reported procedure: ${ }^{8}$ To a stirred solution of $N, N$-diisopropylamine (3.5 $\mathrm{mL}, 25.4 \mathrm{mmol}, 3.0$ equiv.) in dry THF $(10 \mathrm{~mL})$ was added $n$-BuLi $(2.5 \mathrm{M}$ in hexanes, 9.8 $\mathrm{mL}, 24.5 \mathrm{mmol}, 2.9$ equiv.) at $-78^{\circ} \mathrm{C}$. After $30 \mathrm{~min}$ at $-78^{\circ} \mathrm{C}$, (R)-methyl-3-hydroxybutyrate ( $1 \mathrm{~mL}, 8.6 \mathrm{mmol} 1.0$ equiv.) was added dropwise. The resulting mixture was stirred for 30 min at $-78{ }^{\circ} \mathrm{C}$, after which time methyl iodide $(3.13 \mathrm{~mL}, 50.82 \mathrm{mmol}, 6.0$ equiv.) was added. The reaction was stirred at $-78^{\circ} \mathrm{C}$ for further $1.5 \mathrm{~h}$ after which time it was allowed to reach room temperature and aqueous $1 \mathrm{M} \mathrm{HCl}(10 \mathrm{~mL})$ was added. The product was extracted with $\mathrm{CH}_{2} \mathrm{Cl}_{2}(3 \times 10 \mathrm{~mL})$ and the organic layer was washed with brine $(15 \mathrm{~mL})$, dried over $\mathrm{MgSO}_{4}$, filtered and concentrated under reduced pressure. The crude oil was purified by column chromatography on silica gel, eluting with $4: 1$ to $1: 1$ (cyclohexane/EtOAc) to furnish the desired compound as a pale yellow oil ( $888 \mathrm{mg}, 77 \%$ ). ${ }^{1} \mathrm{H} \mathrm{NMR}^{8}$ is consistent with literature. ${ }^{1} \mathrm{H} \mathrm{NMR}\left(400 \mathrm{MHz}, \mathrm{CDCl}_{3}\right) \delta 3.96-3.86(\mathrm{~m}, 1 \mathrm{H})$, $3.74(\mathrm{~s}, 3 \mathrm{H}), 2.64(\mathrm{~d}, J=5.3 \mathrm{~Hz}, 1 \mathrm{H}), 2.49(\mathrm{p}, J=7.2 \mathrm{~Hz}, 1 \mathrm{H}), 1.24(\mathrm{~d}, J=6.3 \mathrm{~Hz}, 3 \mathrm{H}), 1.21$ (d, $J=7.2 \mathrm{~Hz}, 3 \mathrm{H}$ ).

\section{(2S,3R)-3-(benzyloxy)-2-methylbutan-1-ol (S16)}

Using modified general procedure D, ester $\mathbf{S 1 5}(888 \mathrm{mg}, 67.0 \mathrm{mmol}$ ) and LAH $(225 \mathrm{mg}$, $67.0 \mathrm{mmol}$ ) were combined in $\mathrm{Et}_{2} \mathrm{O}(16 \mathrm{~mL})$. Upon workup, the resulting crude oil (700 
$\mathrm{mg}, 67.0 \mathrm{mmol})$, benzaldehyde dimethyl acetal $(1.12 \mathrm{~g}, 7.00 \mathrm{mmol}), p$-toluenesulfonic acid (116 mg, $6.70 \mathrm{mmol})$. The crude reaction mixture was purified using flash column chromatography (5:95 $\mathrm{Et}_{2} \mathrm{O}$ :hexanes) and the resulting benzylidene acetal was isolated as an inseparable mixture of diastereomers and colorless oil ( $1.00 \mathrm{~g}, 77 \%$ over 2 steps).

Using general procedure $\mathbf{E}$, benzylidene acetal $(820 \mathrm{mg}, 4.27 \mathrm{mmol}), \mathrm{BH}_{3} \bullet \mathrm{THF}(21.0 \mathrm{~mL}$, $21.0 \mathrm{mmol})$, and $\mathrm{Cu}(\mathrm{OTf})_{2}(77 \mathrm{mg}, 0.200 \mathrm{mmol})$, were combined to afford S16. S16 was purified using flash column chromatography (30:70 $\mathrm{Et}_{2} \mathrm{O}$ :hexanes) and isolated as a colorless oil (779 mg, 83\%). ${ }^{1} \mathrm{H} \mathrm{NMR}^{9}$ is consistent with literature: ${ }^{1} \mathrm{H}$ NMR $(400 \mathrm{MHz}$, $\left.\mathrm{CDCl}_{3}\right) \delta 7.42-7.30(\mathrm{~m}, 5 \mathrm{H}), 4.68(\mathrm{~d}, J=11.5 \mathrm{~Hz}, 1 \mathrm{H}), 4.44(\mathrm{~d}, J=11.6 \mathrm{~Hz}, 1 \mathrm{H}), 3.70-3.56$ $(\mathrm{m}, 2 \mathrm{H}), 3.51(\mathrm{dq}, J=7.4,6.2 \mathrm{~Hz}, 1 \mathrm{H}), 2.90(\mathrm{~s}, 1 \mathrm{H}), 1.81(\mathrm{hd}, J=7.0,3.7 \mathrm{~Hz}, 1 \mathrm{H}), 1.28(\mathrm{~d}, J$ $=6.1 \mathrm{~Hz}, 3 \mathrm{H}), 0.93(\mathrm{~d}, J=7.0 \mathrm{~Hz}, 3 \mathrm{H})$.

\section{(2R,3R)-3-(benzyloxy)-2-methylbutanal (8f)}

Using general procedure F, alcohol S16 (168 mg, $0.860 \mathrm{mmol}$ ) and DMP (404 mg, 0.950 mmol) were combined in $\mathrm{CH}_{2} \mathrm{Cl}_{2}(8.6 \mathrm{~mL})$ to afford $8 \mathrm{f}$. $8 \mathrm{f}$ was used in subsequent reactions with no further purification. ${ }^{1} \mathrm{H} \mathrm{NMR}^{10}$ is consistent with literature: ${ }^{1} \mathrm{H} \mathrm{NMR}(400 \mathrm{MHz}$, $\left.\mathrm{CDCl}_{3}\right) 9.74(\mathrm{~d}, \mathrm{~J}=2.3 \mathrm{~Hz}, 1 \mathrm{H}), 7.37-7.27(\mathrm{~m}, 5 \mathrm{H}), 4.63(\mathrm{~d}, \mathrm{~J}=11.7 \mathrm{~Hz}, 1 \mathrm{H}), 4.45(\mathrm{~d}, \mathrm{~J}=11.7$ $\mathrm{Hz}, 1 \mathrm{H}), 3.81(\mathrm{p}, \mathrm{J}=6.3 \mathrm{~Hz}, 1 \mathrm{H}), 2.57(\mathrm{pd}, \mathrm{J}=7.1,2.5 \mathrm{~Hz}, 1 \mathrm{H}), 1.25(\mathrm{~d}, \mathrm{~J}=6.2 \mathrm{~Hz}, 3 \mathrm{H}), 1.09$ $(\mathrm{d}, \mathrm{J}=7.2 \mathrm{~Hz}, 3 \mathrm{H})$.

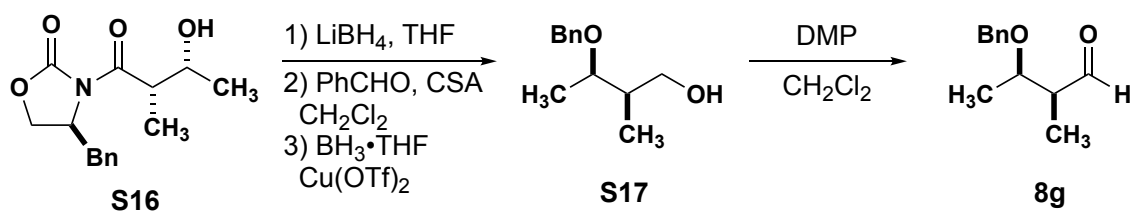

(S)-4-benzyl-3-((2S,3R)-3-hydroxy-2-methylbutanoyl)oxazolidin-2-one (S16)

Known compound $\mathbf{S 1 6}$ was prepared according to previously reported procedure. ${ }^{11}{ }^{1} \mathrm{H}$ $\mathrm{NMR}^{11}$ is consistent with literature. ${ }^{1} \mathrm{H} \mathrm{NMR}\left(400 \mathrm{MHz}, \mathrm{CDCl}_{3}\right) \delta 7.41-7.30(\mathrm{~m}, 3 \mathrm{H}), 7.26$ - $7.18(\mathrm{~m}, 2 \mathrm{H}), 4.74$ (ddt, $J=9.4,7.4,3.3 \mathrm{~Hz}, 1 \mathrm{H}), 3.77$ (qd, J=7.0, $2.9 \mathrm{~Hz}, 1 \mathrm{H}$ ), 3.28 (dd, J $=13.4,3.4 \mathrm{~Hz}, 1 \mathrm{H}), 2.95-2.76(\mathrm{~m}, 2 \mathrm{H}), 1.29(\mathrm{~d}, J=5.6 \mathrm{~Hz}, 3 \mathrm{H}), 1.24(\mathrm{~d}, J=6.4 \mathrm{~Hz}, 3 \mathrm{H})$.

\section{(2R,3R)-3-(benzyloxy)-2-methylbutan-1-ol (S17)}

S16 (1.17 g, $4.20 \mathrm{mmol})$ was dissolved in THF (42 mL) and $\mathrm{MeOH}(0.5 \mathrm{~mL})$. Lithium borohydride (2.0M in THF, $6.30 \mathrm{~mL}$ ) was added dropwise. The reaction was stirred overnight at room temperature and quenched with sat. sodium tartrate $(30 \mathrm{~mL})$. The mixture was stirred for 2 hours at room temperature and then extracted with diethyl ether $(3 \times 30 \mathrm{~mL})$. Combined organics were dried over sodium sulfate, filtered and concentrated in vacuo to produce a crude colorless oil. The crude material was used without further purification and combined with benzaldehyde $(0.857 \mathrm{~mL}, 8.10 \mathrm{mmol})$, camphorsulfonic acid (293 mg, $1.26 \mathrm{mmol}$ ) and magnesium sulfate $(1.52 \mathrm{~g}, 12.6 \mathrm{mmol})$ in DCM $(42 \mathrm{~mL})$. The reaction was stirred overnight at room temperature and then the solids filtered off. The filtrate was washed with sat. sodium bicarbonate and sat. sodium sulfite. 
The organic layer was dried over sodium sulfate, filtered and concentrated in vacuo to produce a colorless oil. The crude material was used without further purification and combined with $\mathrm{BH}_{3} \bullet \mathrm{THF}(1.0 \mathrm{M}$ in THF, $27.35 \mathrm{~mL})$ in DCM $(10 \mathrm{~mL})$ and stirred at room temperature for 10 minutes. Then, copper triflate $(198 \mathrm{mg}, 0.547 \mathrm{mmol})$ was added and the reaction stirred for 2 hours. After 2 hours the reaction was cooled to $0{ }^{\circ} \mathrm{C}$ and 1 equiv. of triethylamine and $\mathrm{MeOH}(5 \mathrm{~mL})$ was added dropwise. The mixture was stirred for 30 minutes and concentrated in vacuo to produce a colorless oil. The crude oil was purified using flash chromatography (10 to $20 \%$ EtOAc in Hexanes) to produce known compound S17 (120 mg, 63\% over three steps). ${ }^{1} \mathrm{H} \mathrm{NMR}^{10}$ is consistent with literature: ${ }^{1} \mathrm{H}$ NMR (599 $\left.\mathrm{MHz}, \mathrm{CDCl}_{3}\right) \delta 7.39-7.26(\mathrm{~m}, 5 \mathrm{H}), 4.61(\mathrm{~d}, J=11.8 \mathrm{~Hz}, 1 \mathrm{H}), 4.45(\mathrm{~d}, J=11.8 \mathrm{~Hz}, 1 \mathrm{H}), 3.70$ (ddt, $J=10.9,7.3,4.0 \mathrm{~Hz}, 2 \mathrm{H}), 3.55(\mathrm{dt}, J=10.9,5.3 \mathrm{~Hz}, 1 \mathrm{H}), 2.65-2.57(\mathrm{~m}, 1 \mathrm{H}), 2.00(\mathrm{tt}$, $J=7.5,4.1 \mathrm{~Hz}, 1 \mathrm{H}), 1.20(\mathrm{dd}, J=6.4,1.8 \mathrm{~Hz}, 3 \mathrm{H}), 0.87(\mathrm{dd}, J=7.2,1.8 \mathrm{~Hz}, 3 \mathrm{H})$.

\section{(2S,3R)-3-(benzyloxy)-2-methylbutanal (8g)}

Using general procedure $\mathbf{F}$, alcohol $\mathbf{S 1 7}(120 \mathrm{mg}, 0.618 \mathrm{mmol}$ ) and DMP (314 mg, 0.742 $\mathrm{mmol}$ ) were combined in $\mathrm{CH} 2 \mathrm{Cl} 2(6 \mathrm{~mL})$ to afford $8 \mathrm{~g}$. $8 \mathrm{~g}$ was used in subsequent reactions with no further purification. $1 \mathrm{H} \mathrm{NMR}^{10}$ is consistent with literature: ${ }^{1} \mathrm{H} \mathrm{NMR}(400 \mathrm{MHz}$, $\left.\mathrm{CDCl}_{3}\right) \delta 9.76(\mathrm{~d}, J=1.2 \mathrm{~Hz}, 1 \mathrm{H}), 7.40-7.27(\mathrm{~m}, 5 \mathrm{H}), 4.61(\mathrm{~d}, J=11.8 \mathrm{~Hz}, 1 \mathrm{H}), 4.46(\mathrm{~d}, J=$ $11.8 \mathrm{~Hz}, 1 \mathrm{H}$ ), 3.96 (qd, $J=6.3,4.3 \mathrm{~Hz}, 1 \mathrm{H}$ ), 2.52 (qdd, $J=7.0,4.4,1.2 \mathrm{~Hz}, 1 \mathrm{H}$ ), 1.26 (d, $J=$ $6.4 \mathrm{~Hz}, 3 \mathrm{H}), 1.14(\mathrm{~d}, J=7.0 \mathrm{~Hz}, 3 \mathrm{H})$.

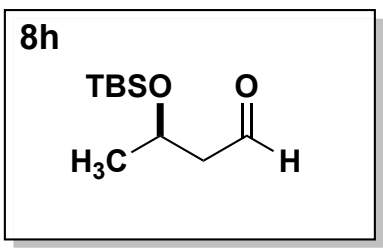

\section{(R)-3-((tert-butyldimethylsilyl)oxy)butanal (8h)}

Known aldehyde $8 \mathrm{~h}$ was prepared according to previously reported procedure. ${ }^{1} \mathrm{H}$ NMR is consistent with literature. ${ }^{1}{ }^{1} \mathrm{H} \mathrm{NMR}\left(400 \mathrm{MHz}, \mathrm{CDCl}_{3}\right) \delta 9.83(\mathrm{t}, J=2.3 \mathrm{~Hz}, 1 \mathrm{H}), 7.34$ ( $q d, J=8.6,7.6,4.1 \mathrm{~Hz}, 5 \mathrm{H}), 4.63-4.49(\mathrm{~m}, 2 \mathrm{H}), 4.07-3.90(\mathrm{~m}, 1 \mathrm{H}), 2.70$ (ddd, $J=16.3$, $7.2,2.6 \mathrm{~Hz}, 1 \mathrm{H}), 2.59$ (ddd, $J=16.3,4.8,1.9 \mathrm{~Hz}, 1 \mathrm{H}), 1.83-1.66(\mathrm{~m}, 1 \mathrm{H}), 1.66-1.52(\mathrm{~m}$, $1 \mathrm{H}), 1.37(\mathrm{qd}, J=10.5,9.6,6.4 \mathrm{~Hz}, 4 \mathrm{H}), 0.97-0.89(\mathrm{~m}, 3 \mathrm{H})$.

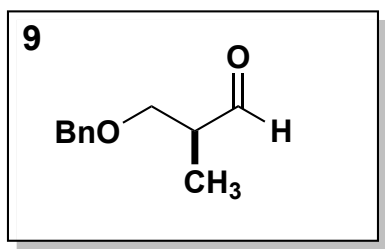

\section{(S)-3-(benzyloxy)-2-methylpropanal (9)}


Known aldehyde 9 was prepared according to previously reported procedure. ${ }^{12}{ }^{1} \mathrm{H}$ NMR is consistent with literature. ${ }^{12}{ }^{1} \mathrm{H}$ NMR $(599 \mathrm{MHz}$, Chloroform- $d) \delta 9.72(\mathrm{~d}, J=1.7 \mathrm{~Hz}, 1 \mathrm{H})$, $7.32(\mathrm{dq}, J=15.8,7.7 \mathrm{~Hz}, 5 \mathrm{H}), 4.52(\mathrm{~s}, 3 \mathrm{H}), 3.71-3.60(\mathrm{~m}, 3 \mathrm{H}), 2.66$ (tdd, $J=7.1,5.3,1.7$ $\mathrm{Hz}, 1 \mathrm{H}), 1.13(\mathrm{~d}, J=7.2 \mathrm{~Hz}, 3 \mathrm{H})$.

\section{References}

1. Ren, H.; Wulff, W. D., Total Synthesis of Sedum Alkaloids Via Catalyst Controlled Aza-Cope Rearrangement and Hydroformylation with Formaldehyde. Org. Lett. 2013, 15 (2), 242-245.

2. Kano, T.; Tanaka, Y.; Maruoka, K., Asymmetric Organocatalytic Oxy-Michael Addition of Alcohols to A,B-Unsaturated Aldehydes. Tetrahedron 2007, 63 (35), 8658-8664.

3. Harada, T.; Egusa, T.; Igarashi, Y.; Kinugasa, M.; Oku, A., Inter- and Intramolecular Differentiation of Enantiotopic Dioxane Acetals through OxazaborolidinoneMediated Enantioselective Ring-Cleavage Reaction: Kinetic Resolution of Racemic 1,3-Alkanediols and Asymmetric Desymmetrization of Meso-1,3Polyols. J. Org. Chem. 2002, 67 (20), 7080-7090.

4. Hiroyuki, I.; Hiroki, S.; Hideo, K.; Kiyoyuki, Y., Enantioselective Total Synthesis of Doliculide, a Potent Cytotoxic Cyclodepsipeptide of Marine Origin and StructureCytotoxicity Relationships of Synthetic Doliculide Congeners. Tetrahedron 1994, 50 (45), 12853-12882.

5. Merkley, N.; Warkentin, J., Thermolysis of a Spiro-Fused Oxadiazoline: The Carbonyl Ylide Cyclic Carbene Diradical Sequence. Can. J. Chem. 2002, 80 (9), 11871195.

6. Tatina, M.; Yousuf, S. K.; Aravinda, S.; Singh, B.; Mukherjee, D., Cyanuric Chloride/Sodium Borohydride: A New Reagent Combination for Reductive Opening of 4,6-Benzylidene Acetals of Carbohydrates to Primary Alcohols. Carbohydr. Res. 2013, 381, 142-5.

7. Hoffmann, R.; Brückner, R., Asymmetric Induction in Reductively Initiated [2,3]-Wittig and Retro [1,4]-Brook Rearrangements of Secondary Carbanions. Chem. Ber. 1992, 125 (6), 1471-1484.

8. Makrerougras, M.; Coffinier, R.; Oger, S.; Chevalier, A.; Sabot, C.; Franck, X., Total Synthesis and Structural Revision of Chaetoviridins A. Org. Lett. 2017, 19 (15), 4146-4149.

9. Bosse, K.; Marineau, J.; Nason, D. M.; Fliri, A. J.; Segelstein, B. E.; Desai, K.; Volkmann, R. A., Expanding the Medicinal Chemistry Toolbox: Stereospecific Generation of Methyl Group-Containing Propylene Linkers. Tetrahedron Lett. 2006, 47 (41), 7285-7287.

10. Skotnitzki, J.; Spessert, L.; Knochel, P., Regio- and Stereoselective Allylic Substitutions of Chiral Secondary Alkylcopper Reagents: Total Synthesis of $(+)-$ Lasiol, (+)-13-Norfaranal, and (+)-Faranal. Angew. Chem., Int. Ed. 2019, 58 (5), 1509-1514.

11. Schläger, N.; Kirschning, A., Substrate-Controlled Stereoselectivity in the Yamamoto Aldol Reaction. Org. Bio. Chem. 2012, 10 (38), 7721-7729. 
12. Zanato, C.; Pignataro, L.; Hao, Z.; Gennari, C., A Practical Synthesis of the C1-C9 Fragment of Dictyostatin. Synthesis 2008, 2008 (14), 2158-2162. 


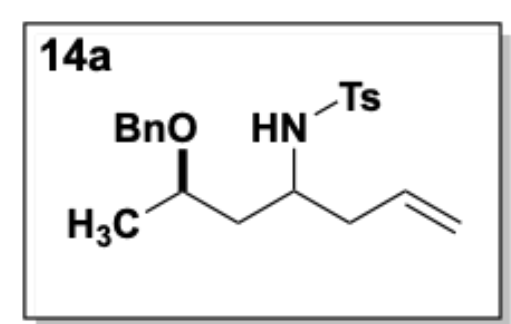

${ }^{1} \mathrm{H}$ NMR (599 MHz, Chloroform-d)
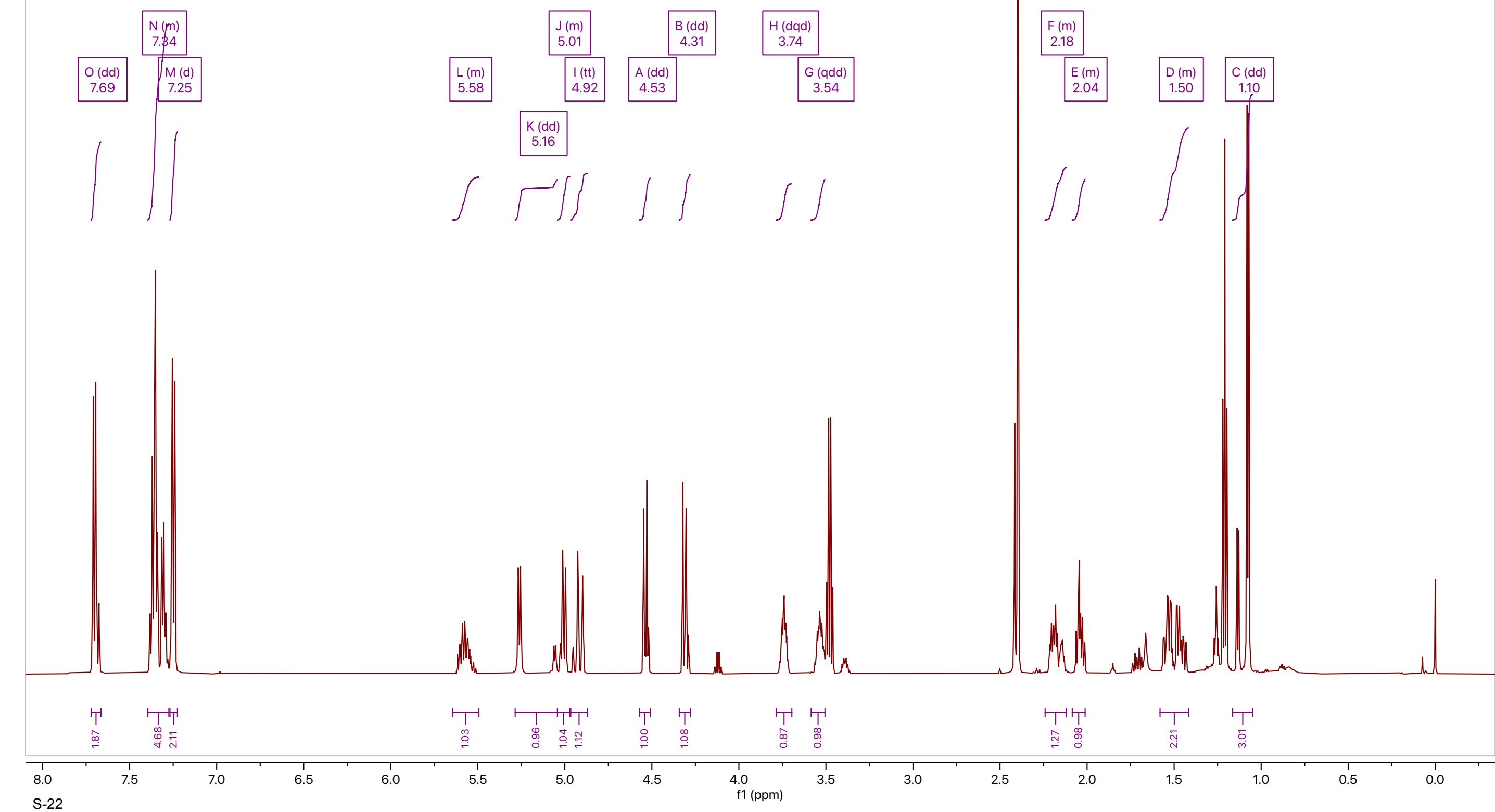


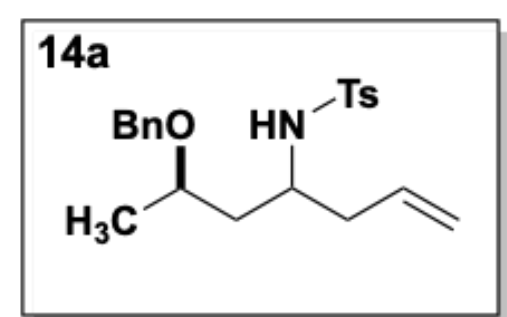

等

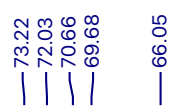

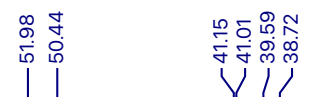

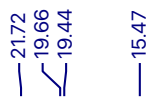

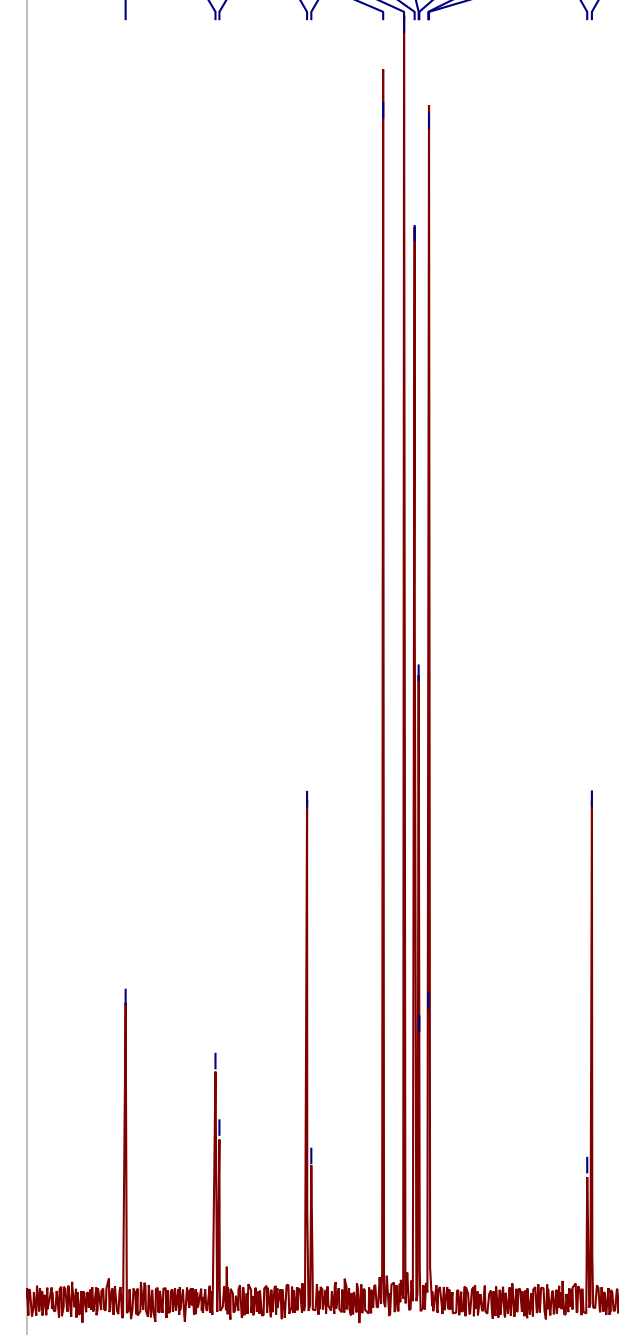



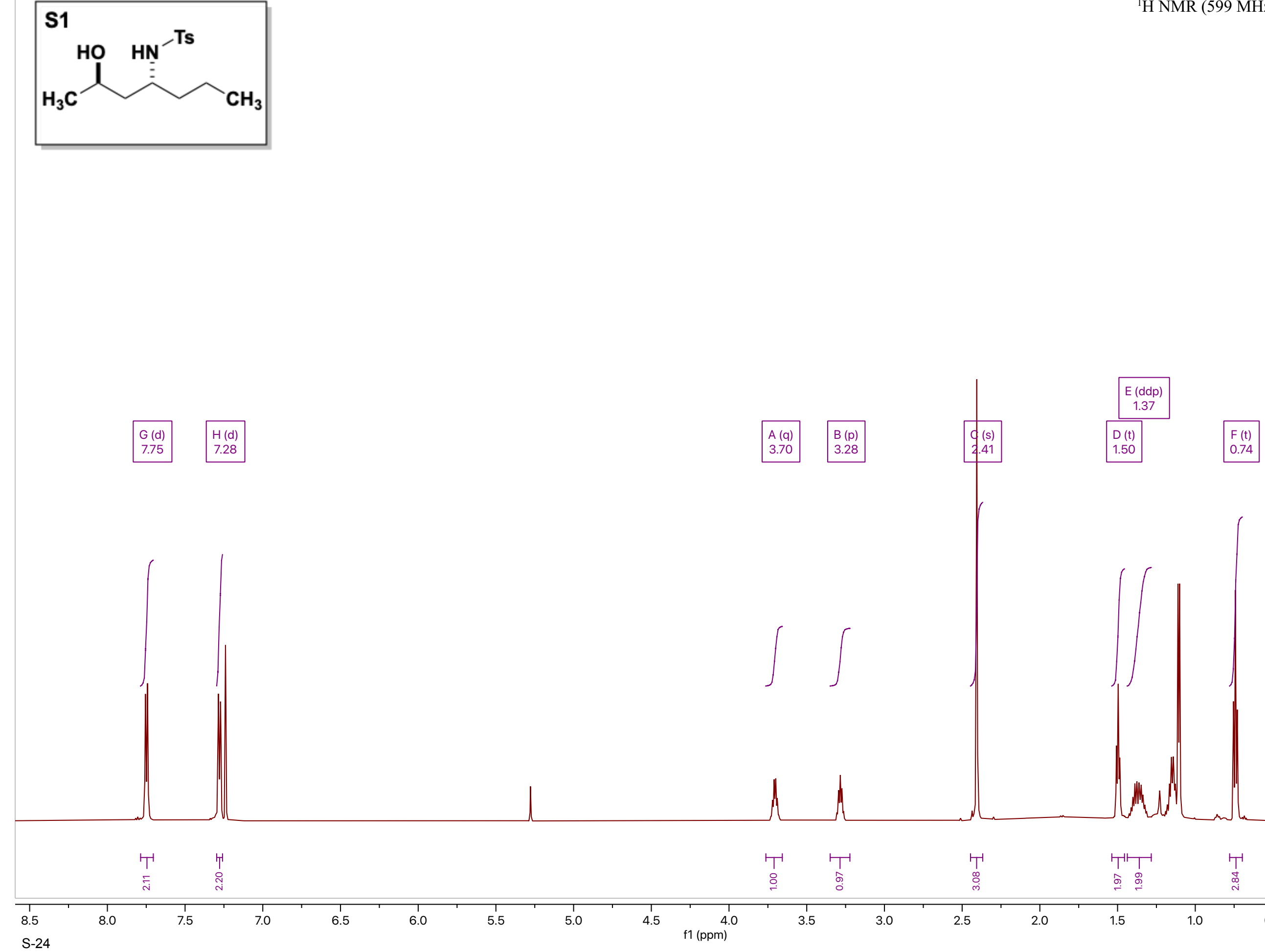
S1

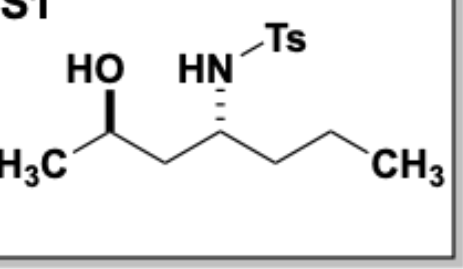

${ }^{13} \mathrm{C}\{1 \mathrm{H}\}$ NMR $\left(151 \mathrm{MHz}, \mathrm{cdcl}_{3}\right)$

|

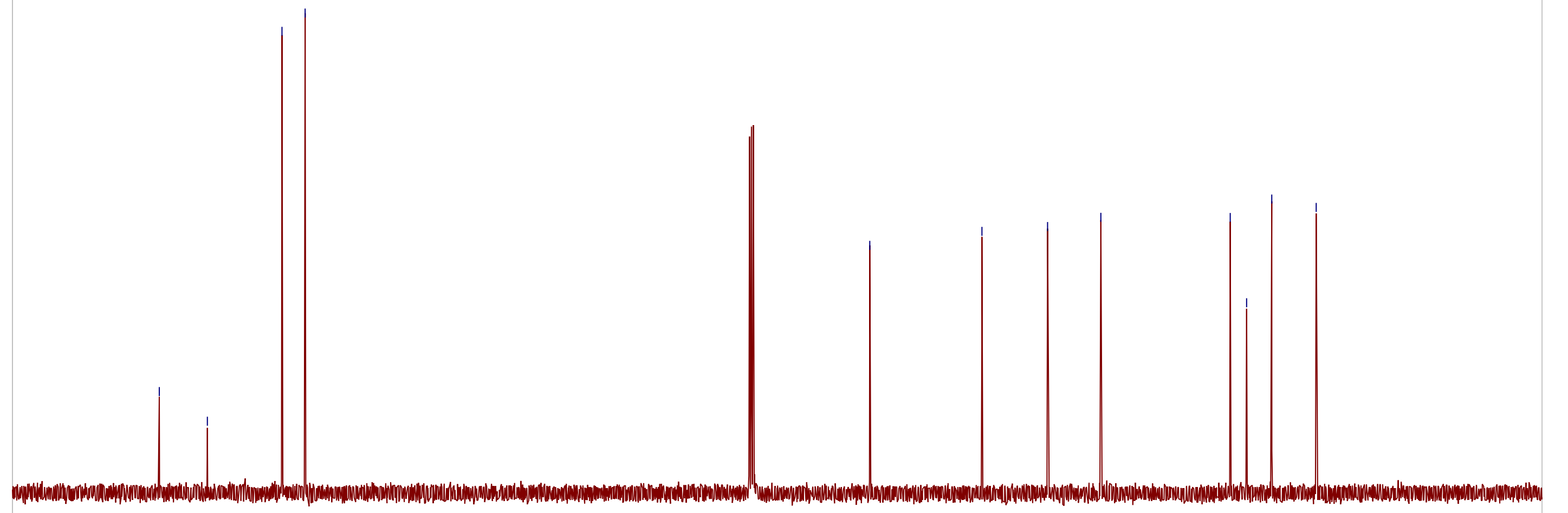




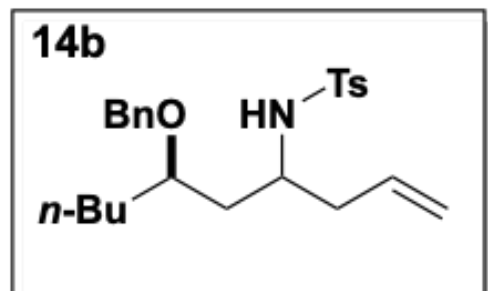

${ }^{1} \mathrm{H}$ NMR (400 MHz, Chloroform- $d$ )

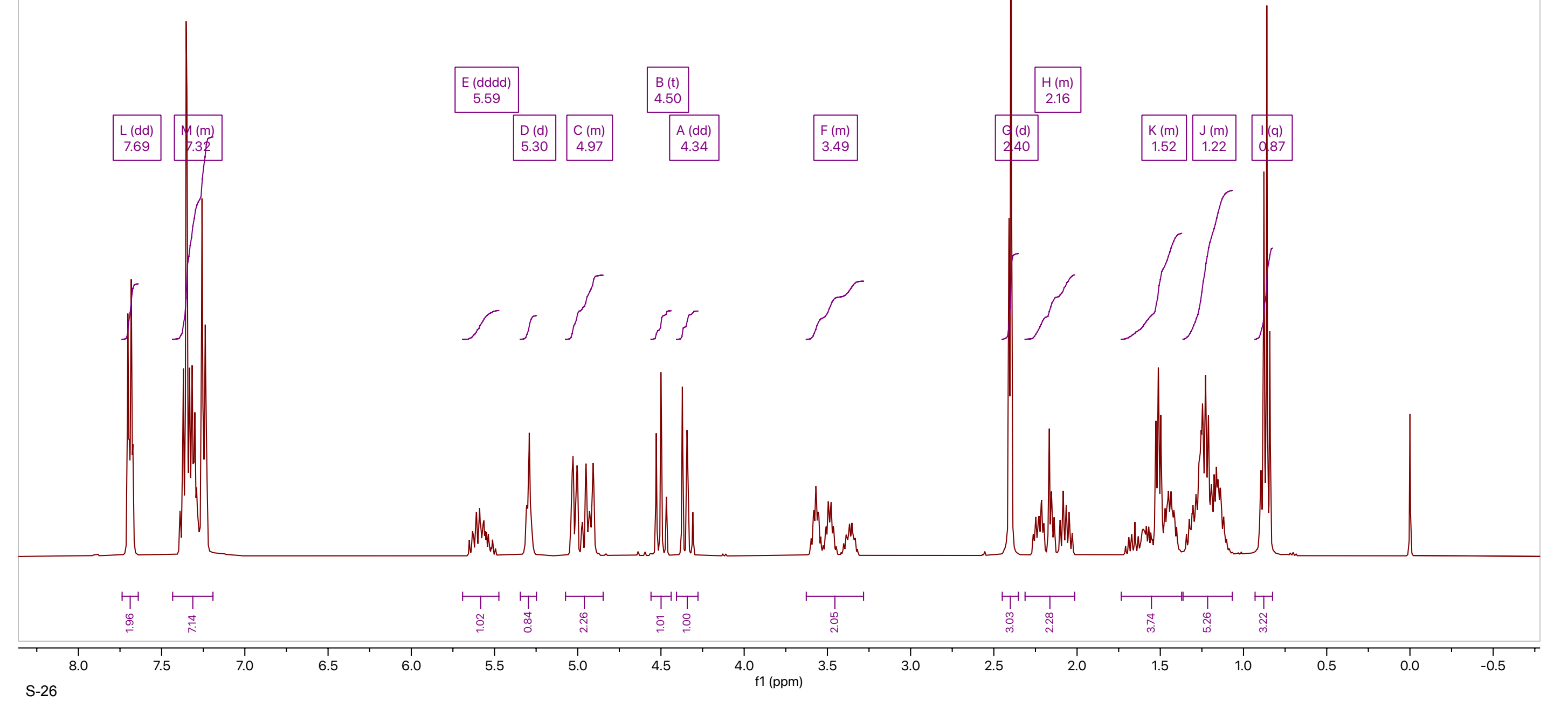


$14 b$

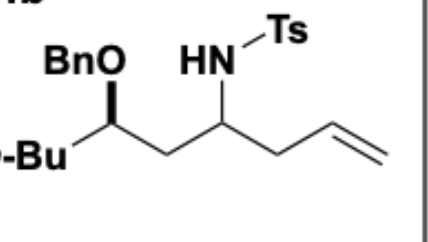

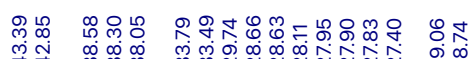

$\bigcup^{1} \underbrace{\infty}$
${ }^{13} \mathrm{C}\{1 \mathrm{H}\}$ NMR $\left(101 \mathrm{MHz}, \mathrm{CDCl}_{3}\right)$

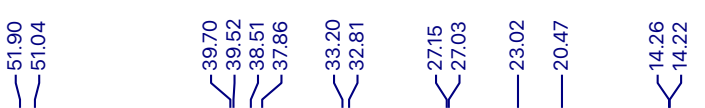

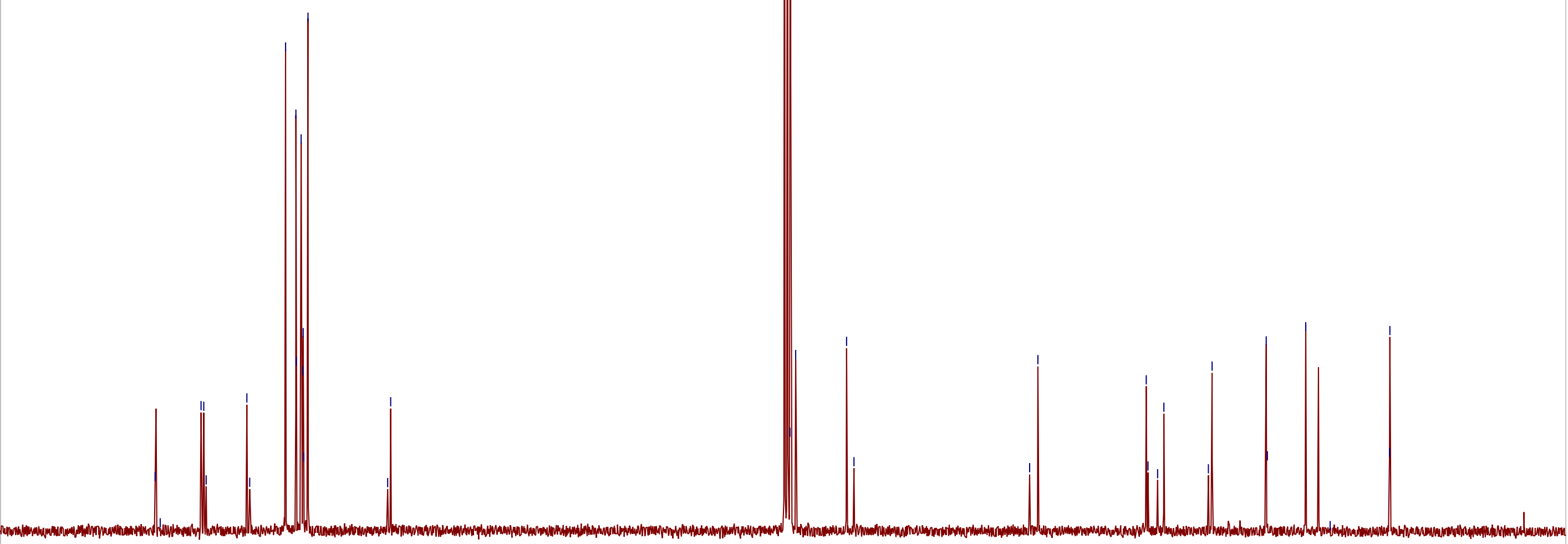




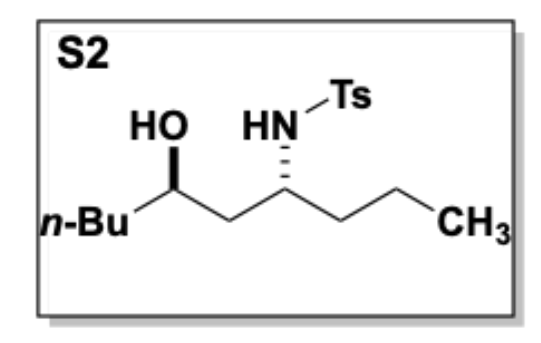

${ }^{1} \mathrm{H}$ NMR (400 MHz, $\mathrm{CDCl}_{3}$ )

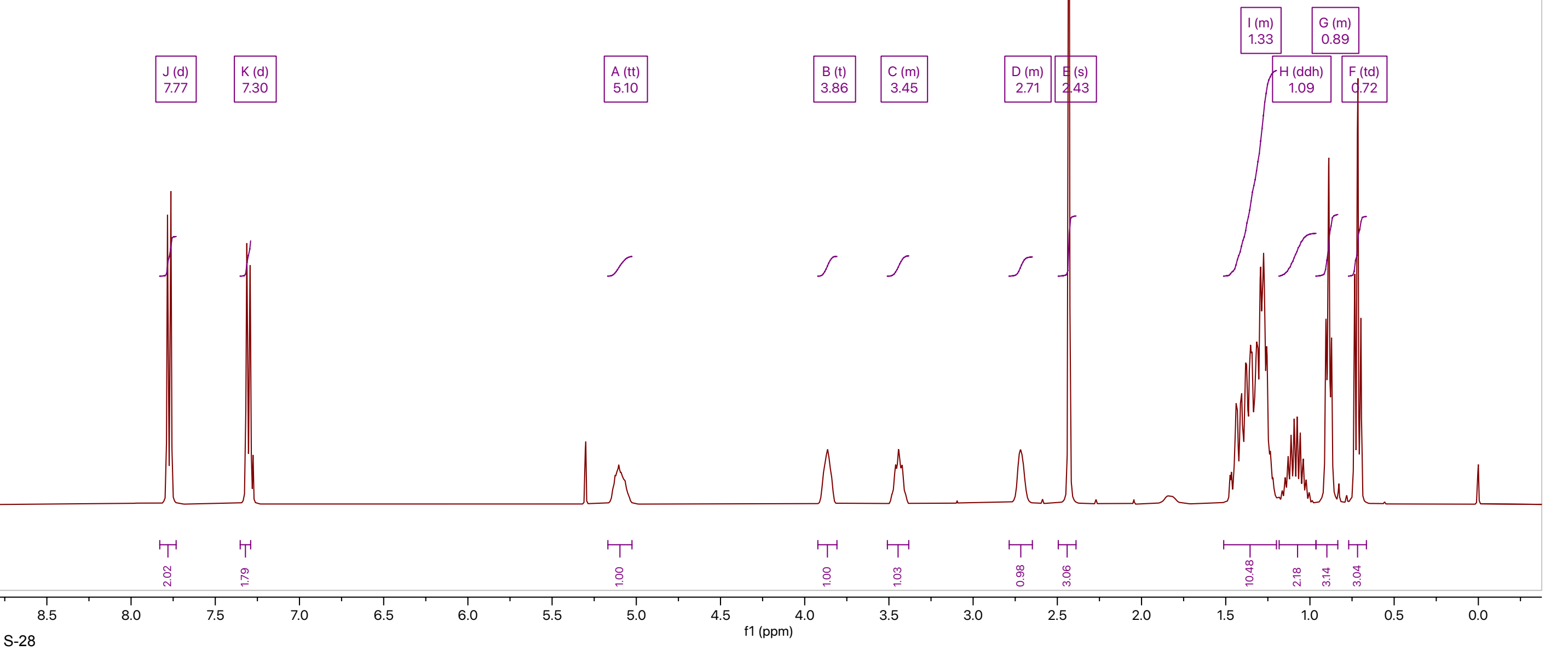




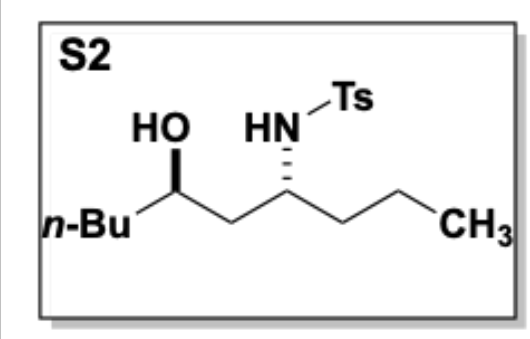

${ }^{13} \mathrm{C}\{1 \mathrm{H}\}$ NMR (101 MHz, $\left.\mathrm{CDCl}_{3}\right)$

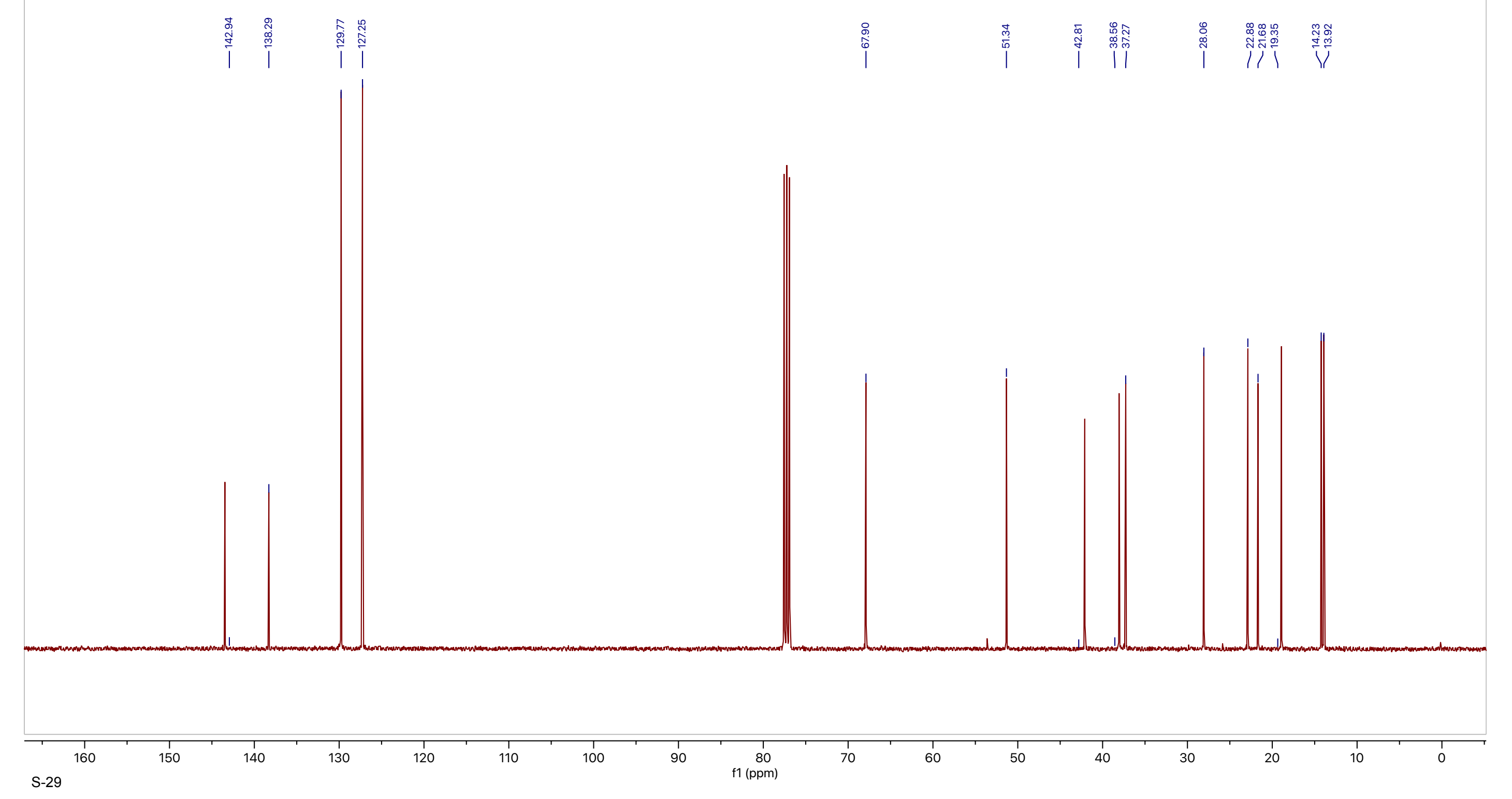




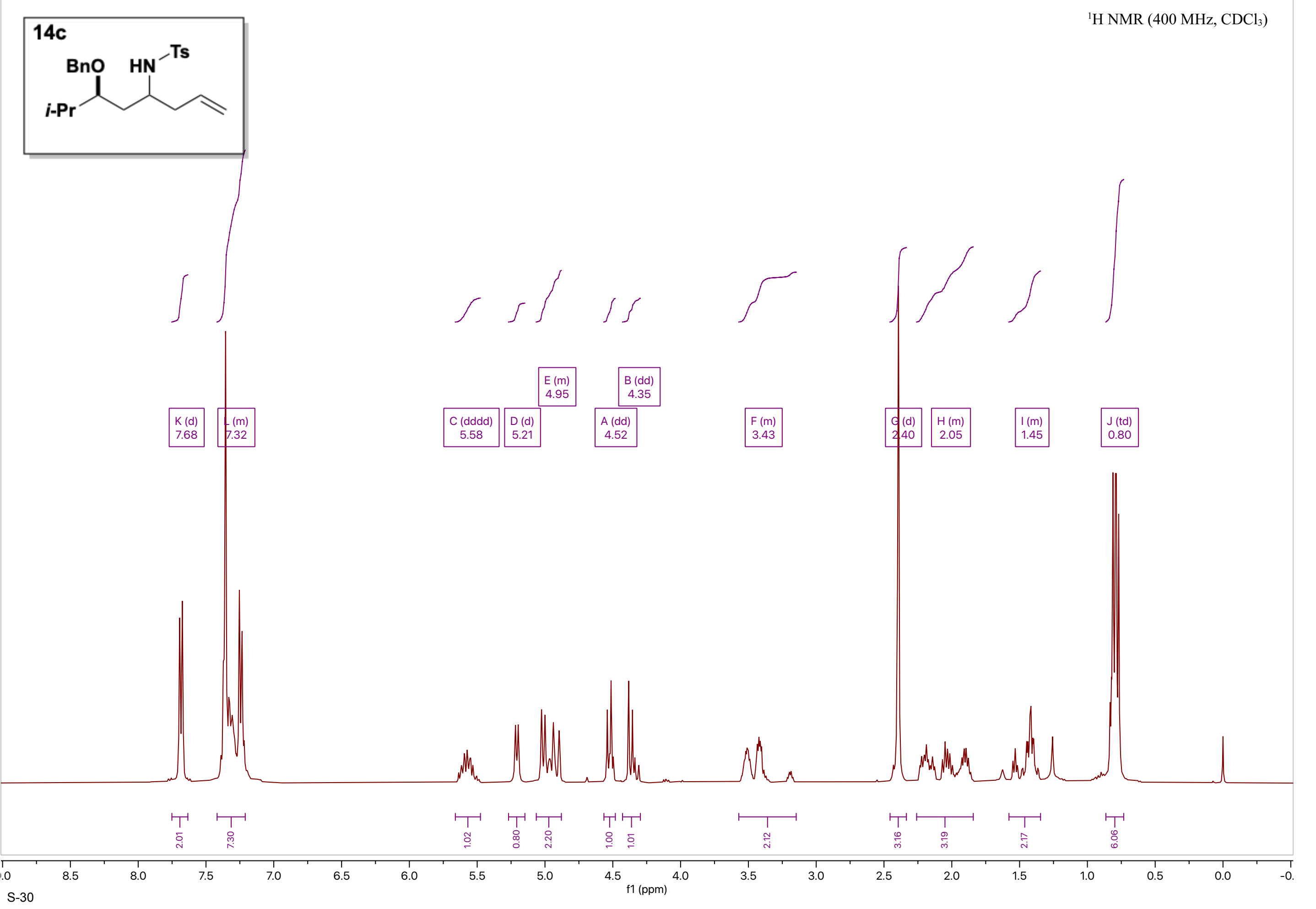




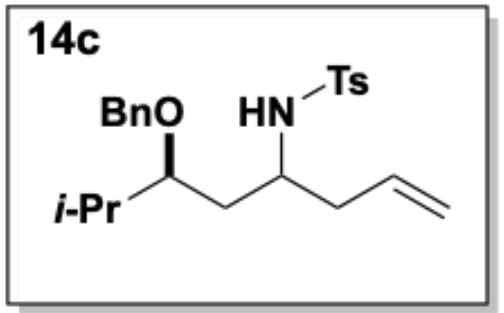

${ }^{13} \mathrm{C}\{1 \mathrm{H}\}$ NMR $\left(101 \mathrm{MHz}, \mathrm{CDCl}_{3}\right)$

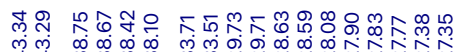

Y vi v vifu

11 ||

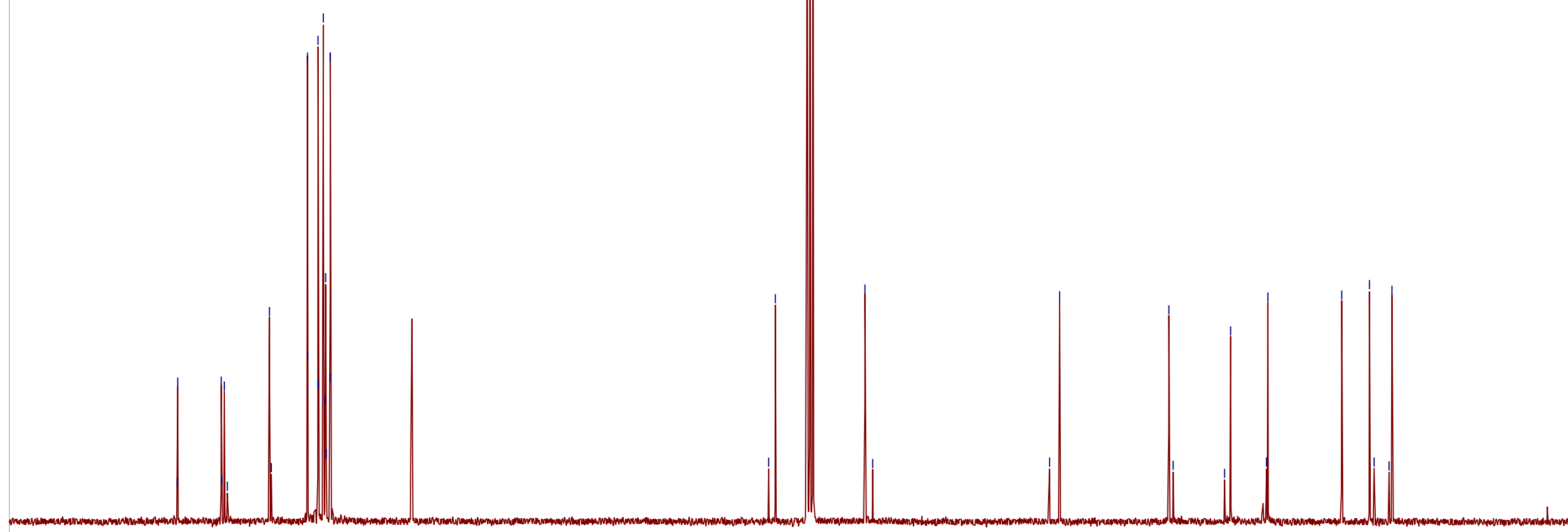

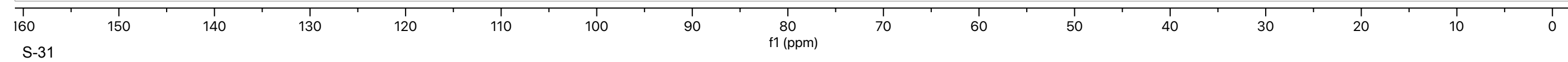




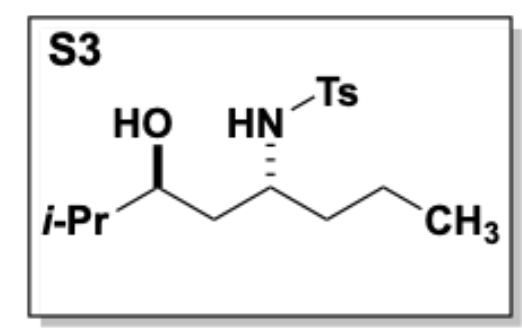

${ }^{1} \mathrm{H}$ NMR (599 MHz, $\mathrm{cdcl}_{3}$ )

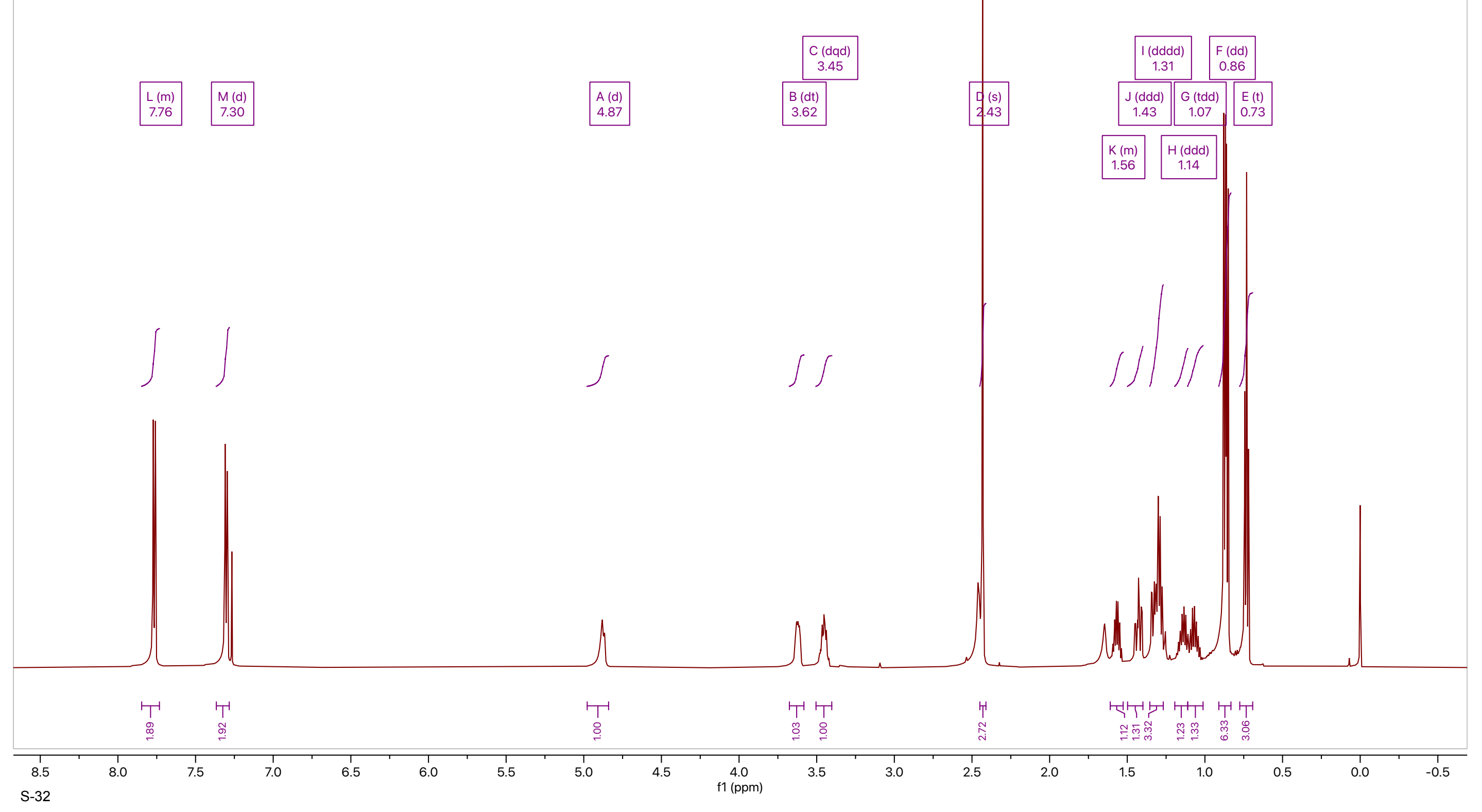



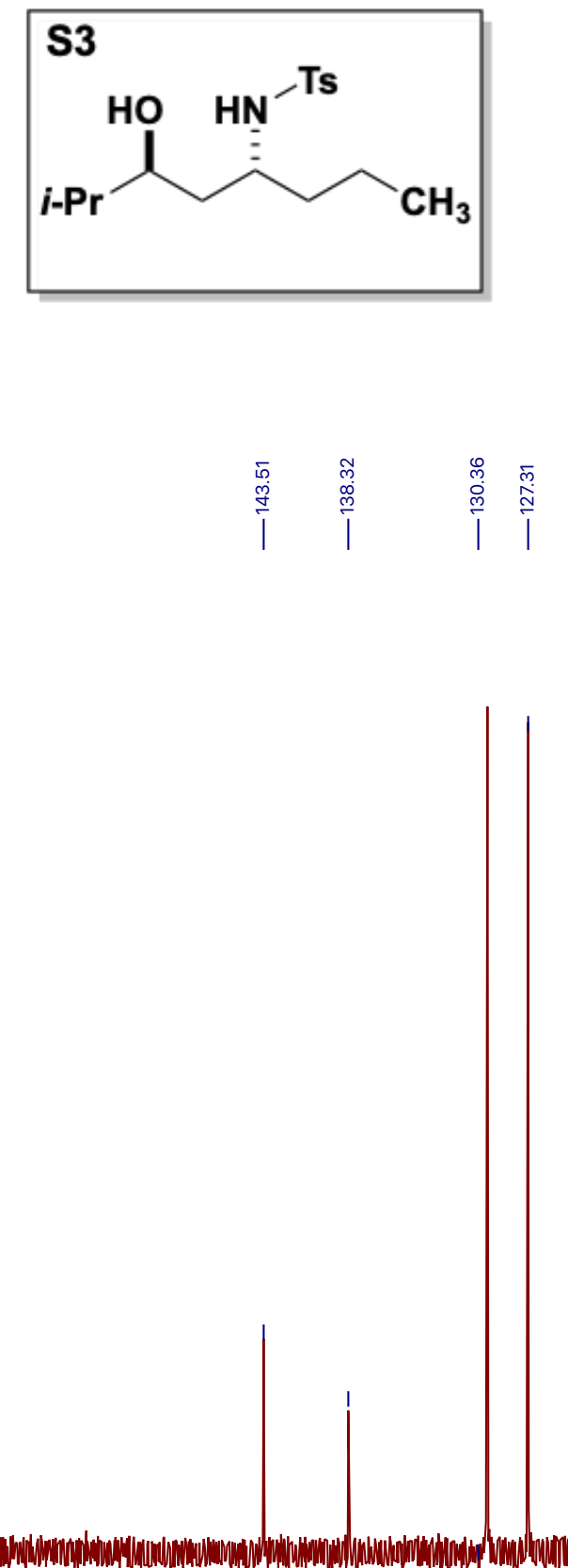

$$
50
$$


14d

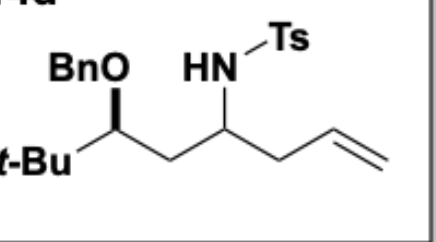

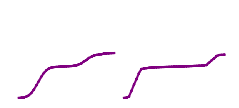
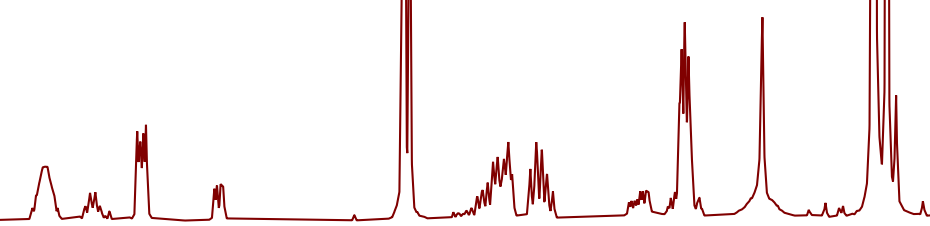

Nhe

$2.40 \quad 2.05$

1.55
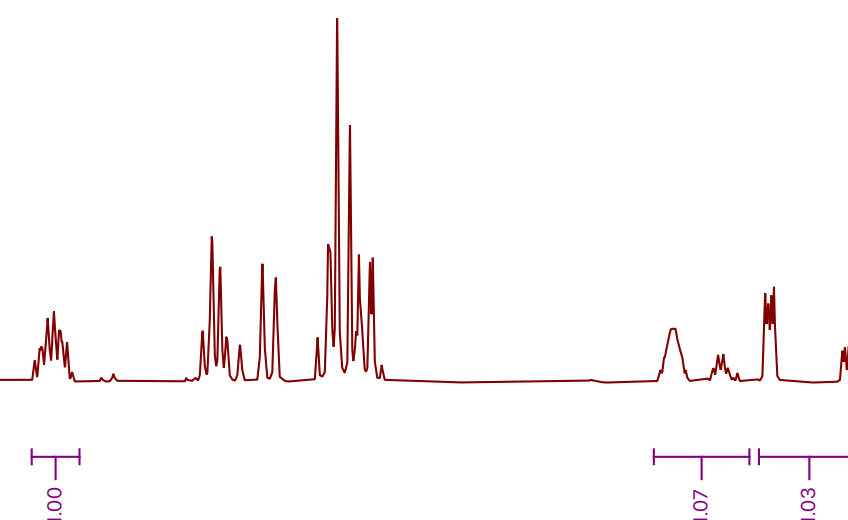

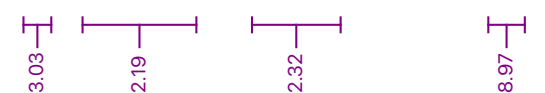


14d

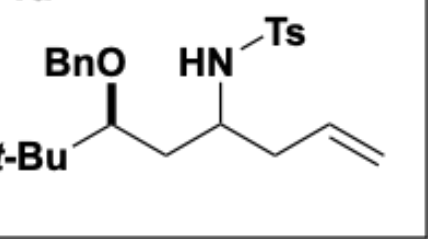

$\bigcup^{1 / 1}$

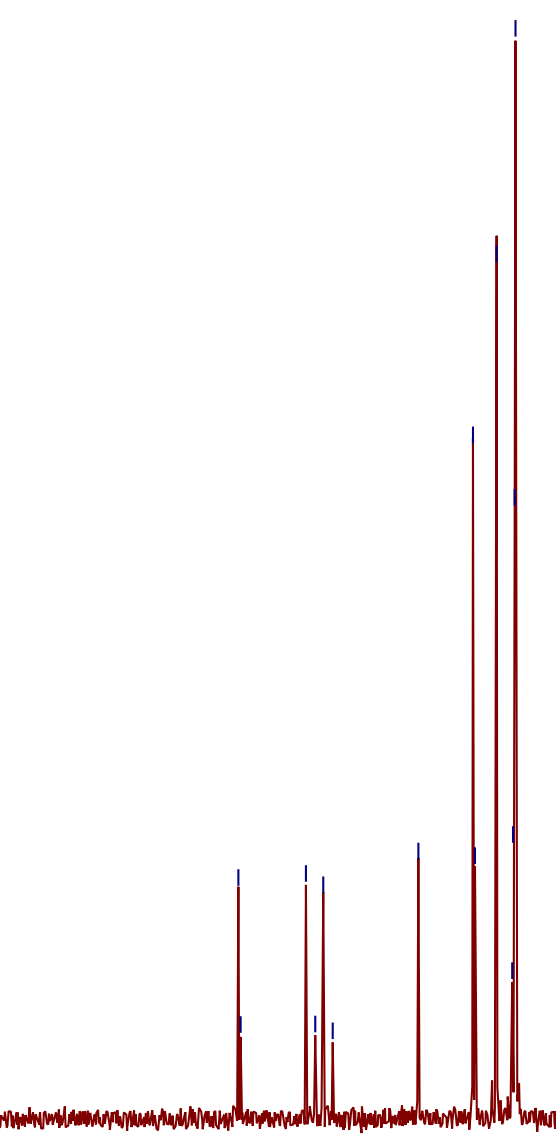

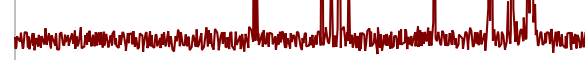

${ }^{13} \mathrm{C}\{1 \mathrm{H}\} \mathrm{NMR}\left(101 \mathrm{MHz}, \mathrm{CDCl}_{3}\right)$

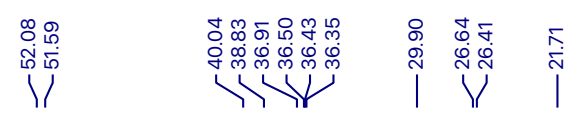

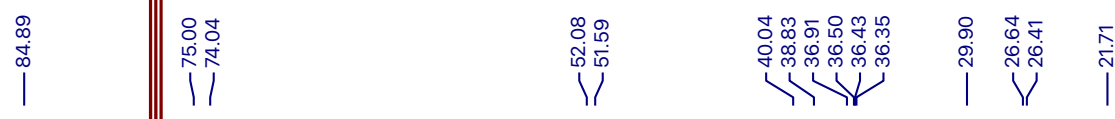

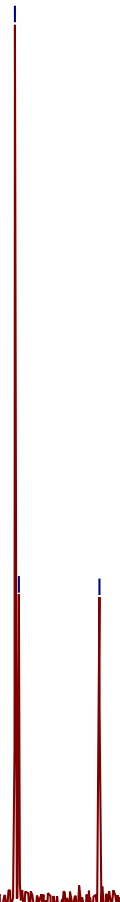



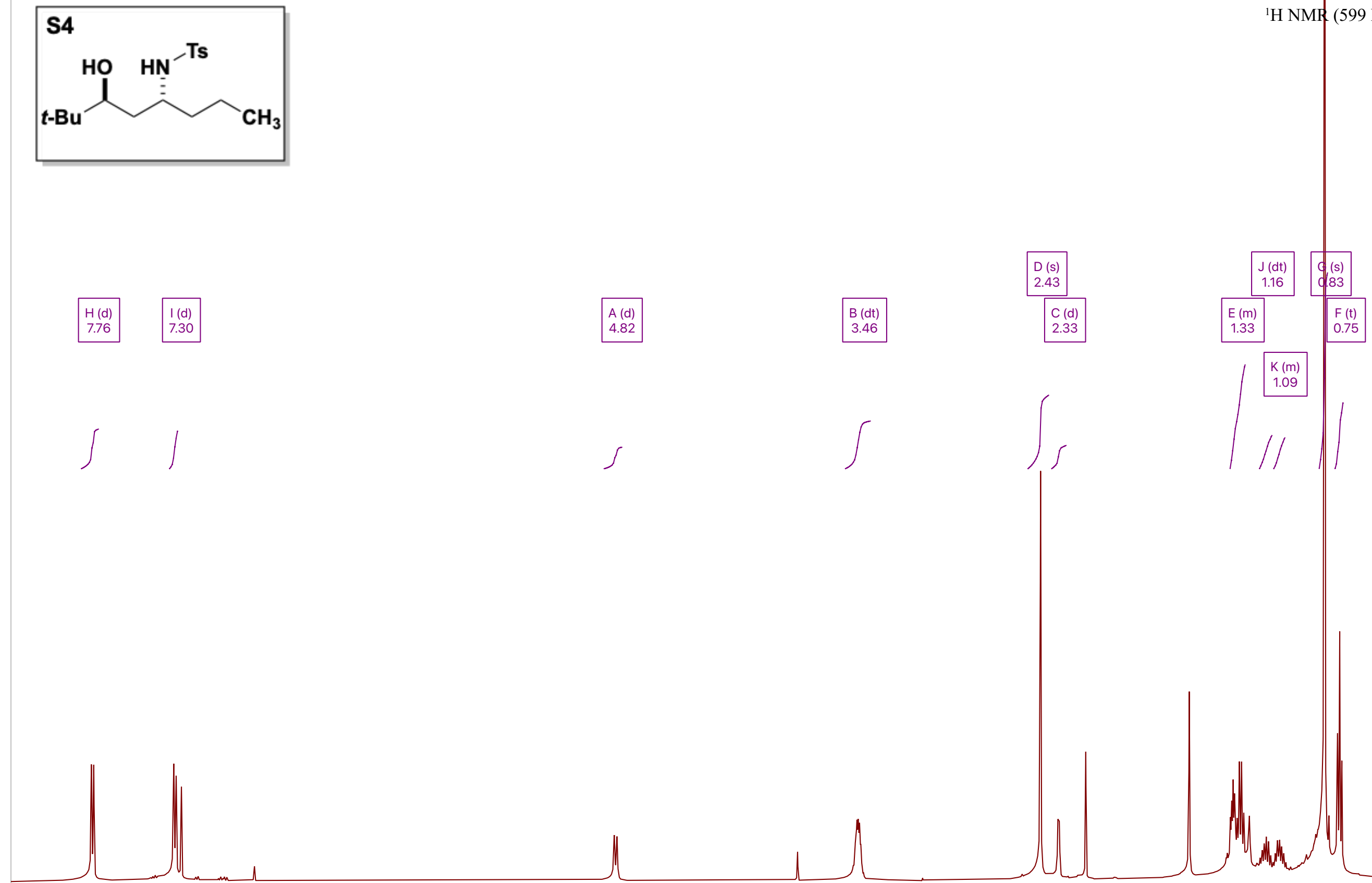


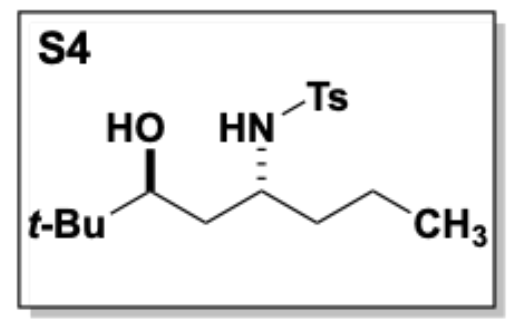

${ }^{13} \mathrm{C}\{1 \mathrm{H}\}$ NMR $\left(151 \mathrm{MHz}, \mathrm{cdcl}_{3}\right)$

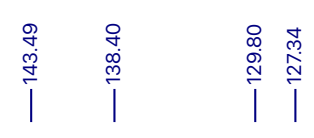

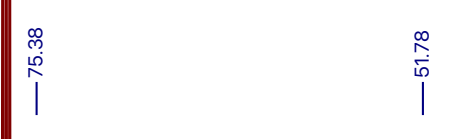

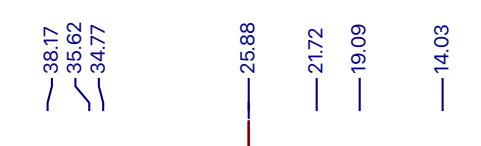

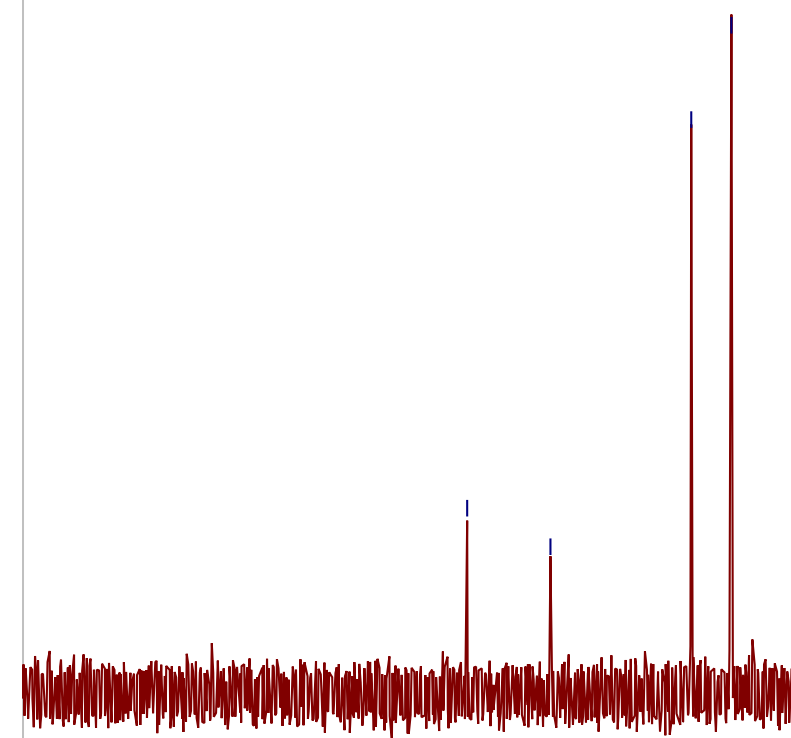

70 


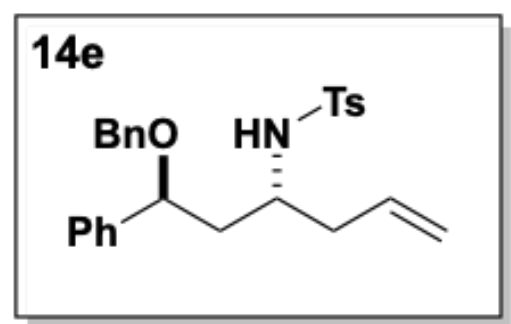

${ }^{1} \mathrm{H}$ NMR (400 MHz, $\mathrm{CDCl}_{3}$ )

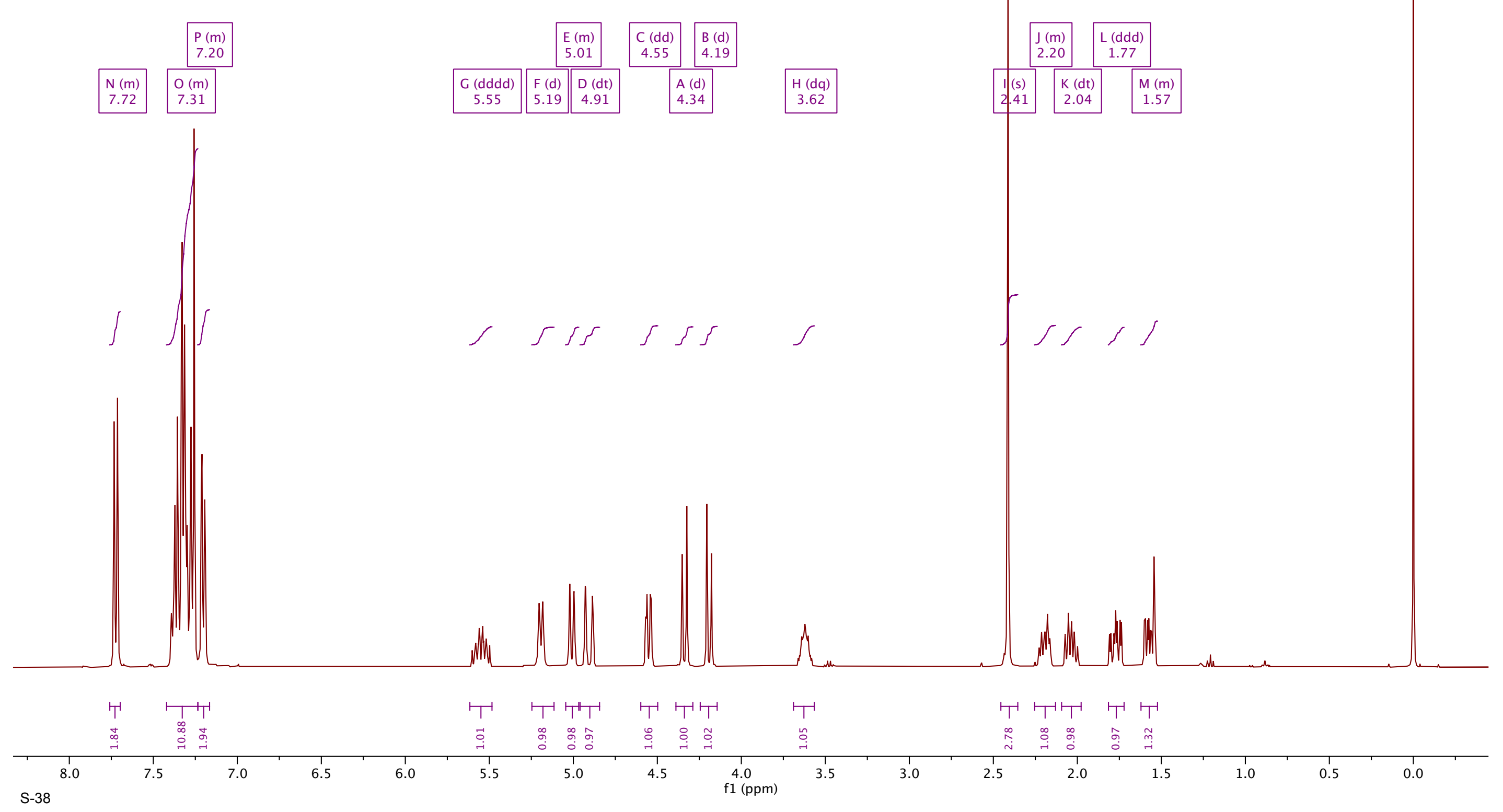



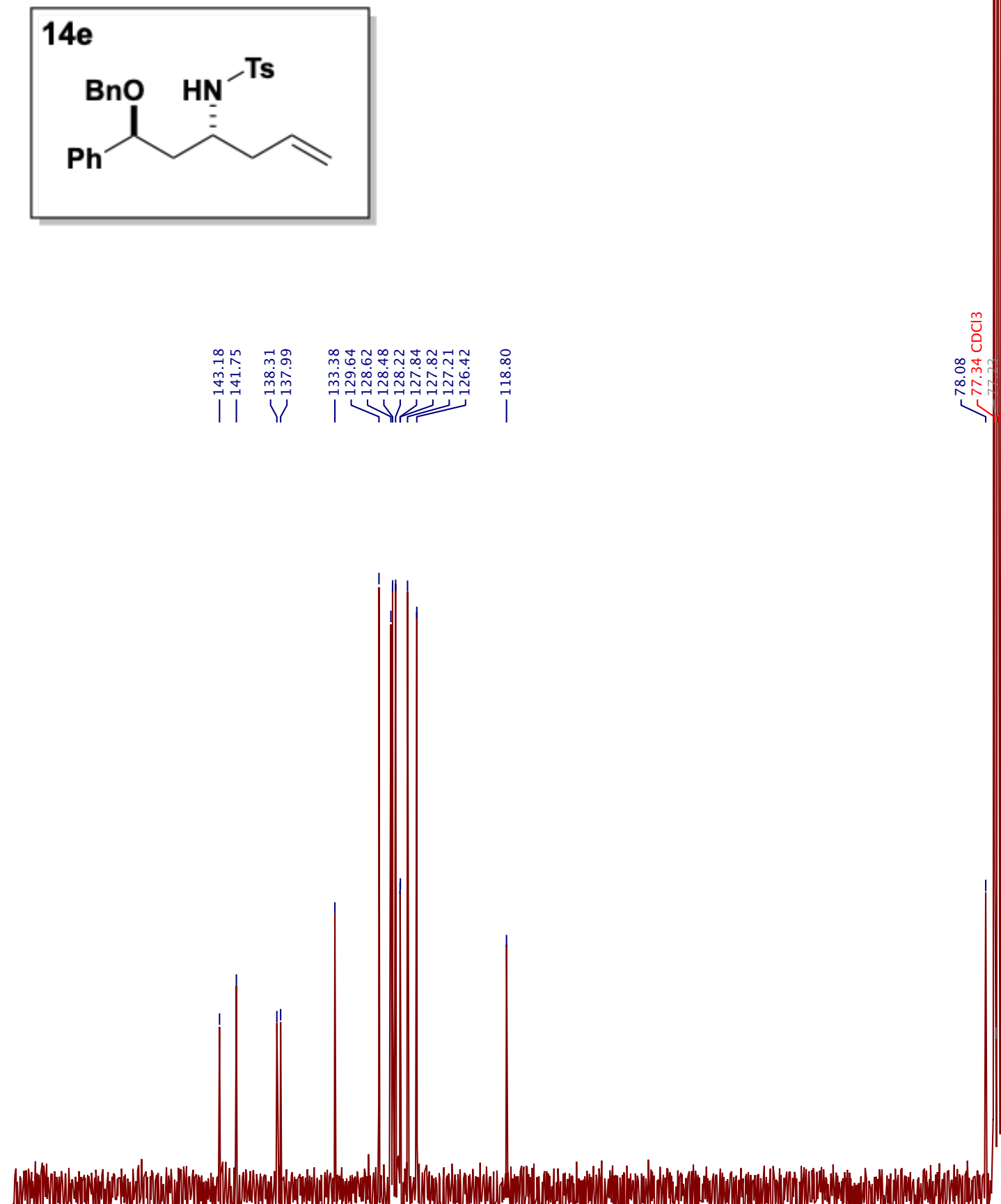


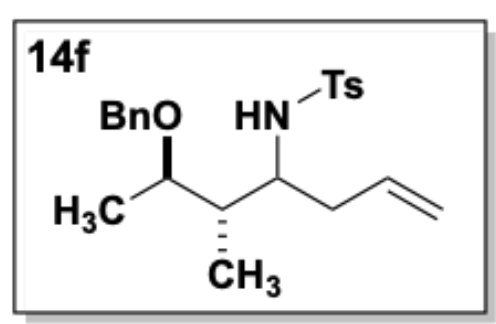

${ }^{1} \mathrm{H}$ NMR (400 MHz, $\mathrm{CDCl}_{3}$ )

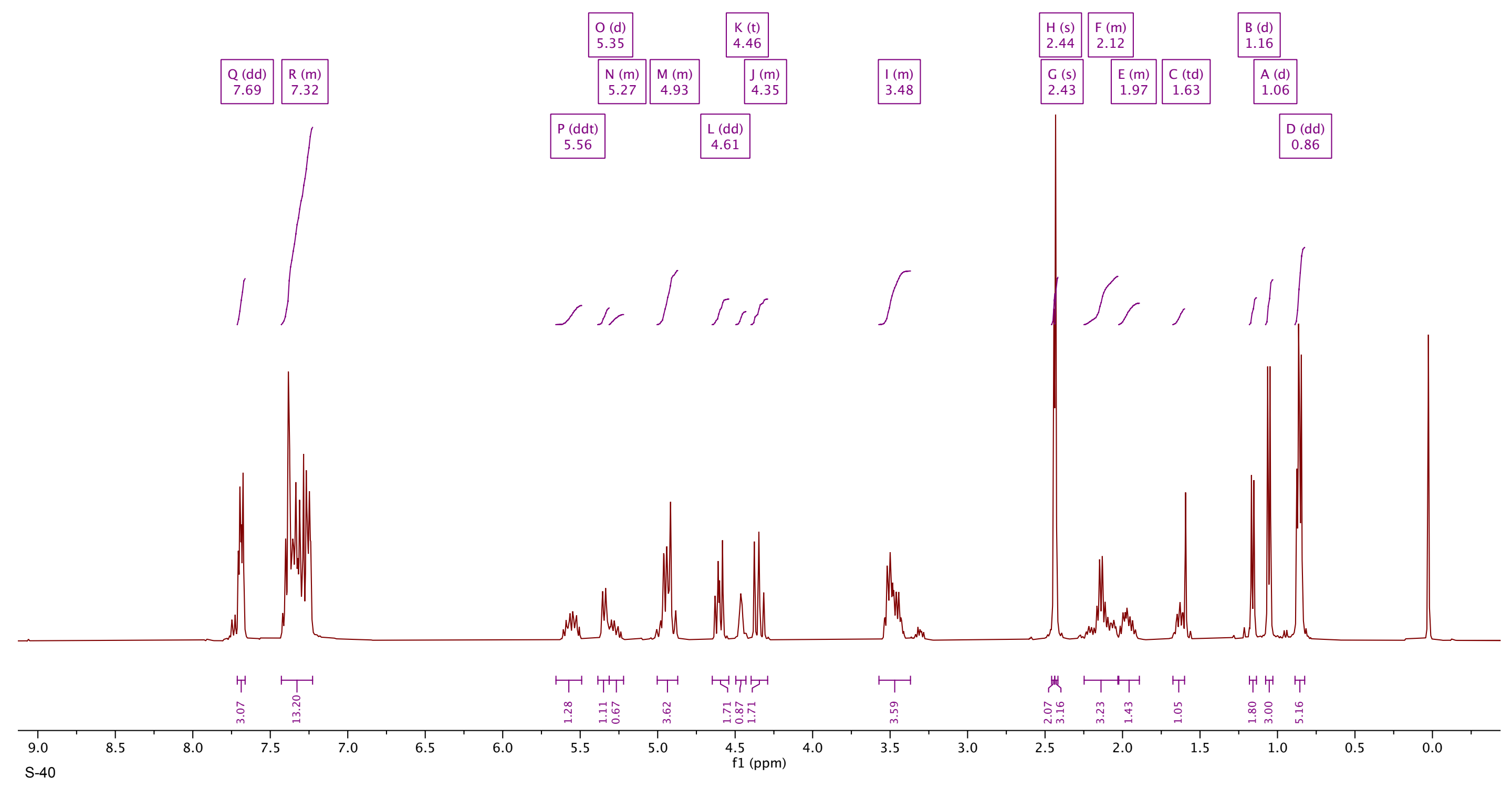




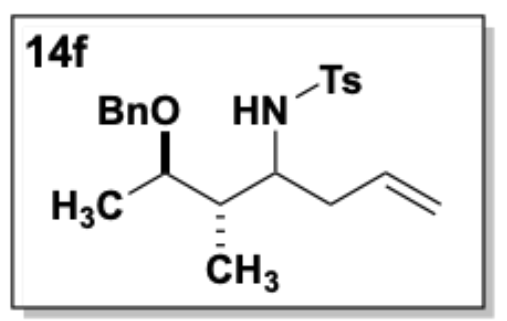

${ }^{13} \mathrm{C}\{1 \mathrm{H}\}$ NMR $\left(101 \mathrm{MHz}, \mathrm{CDCl}_{3}\right)$

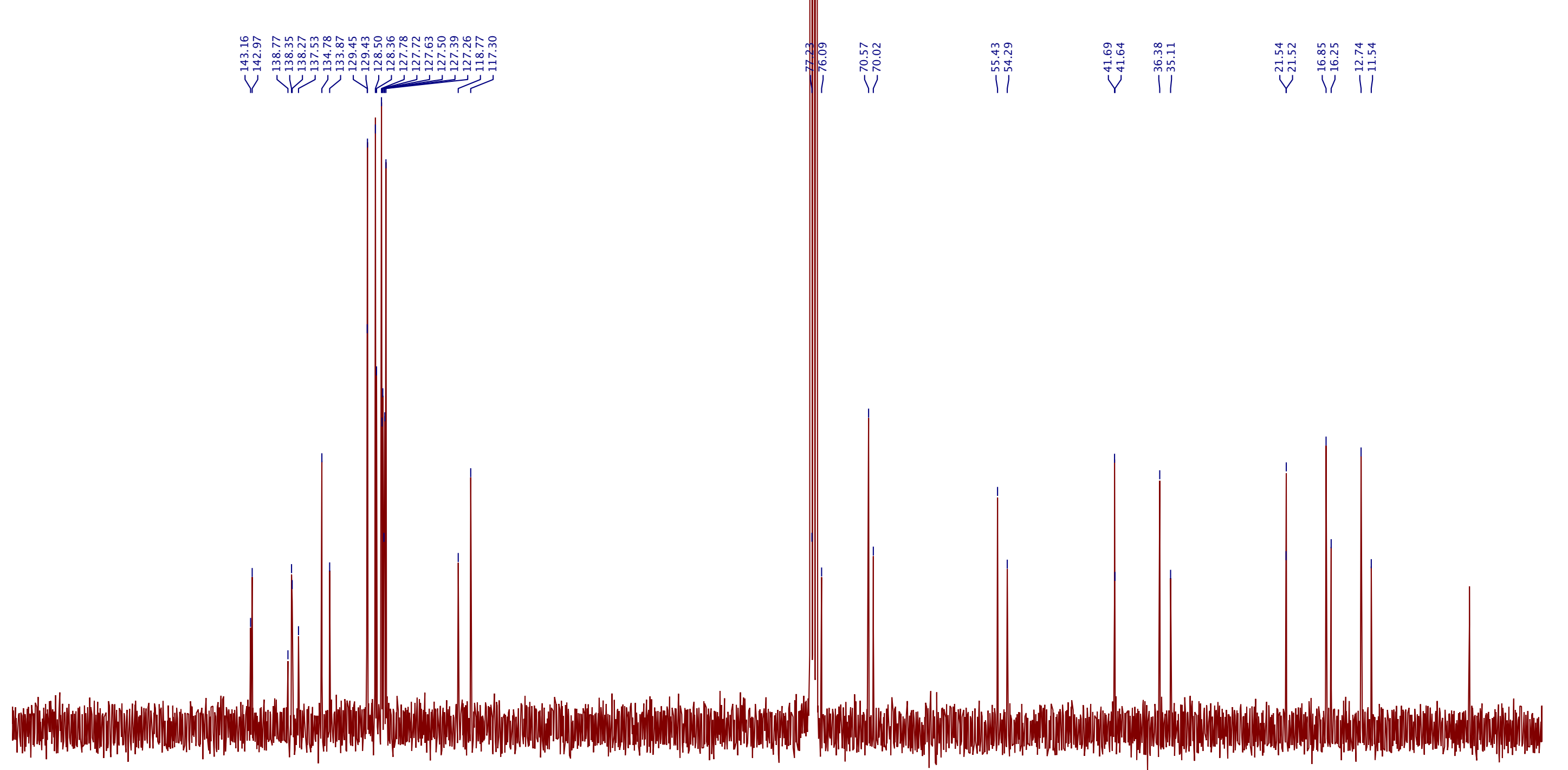

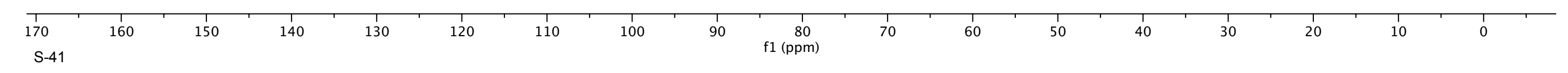




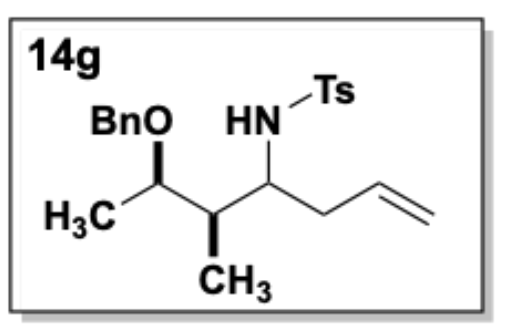

${ }^{1} \mathrm{H}$ NMR (400 MHz, $\mathrm{CDCl}_{3}$ )

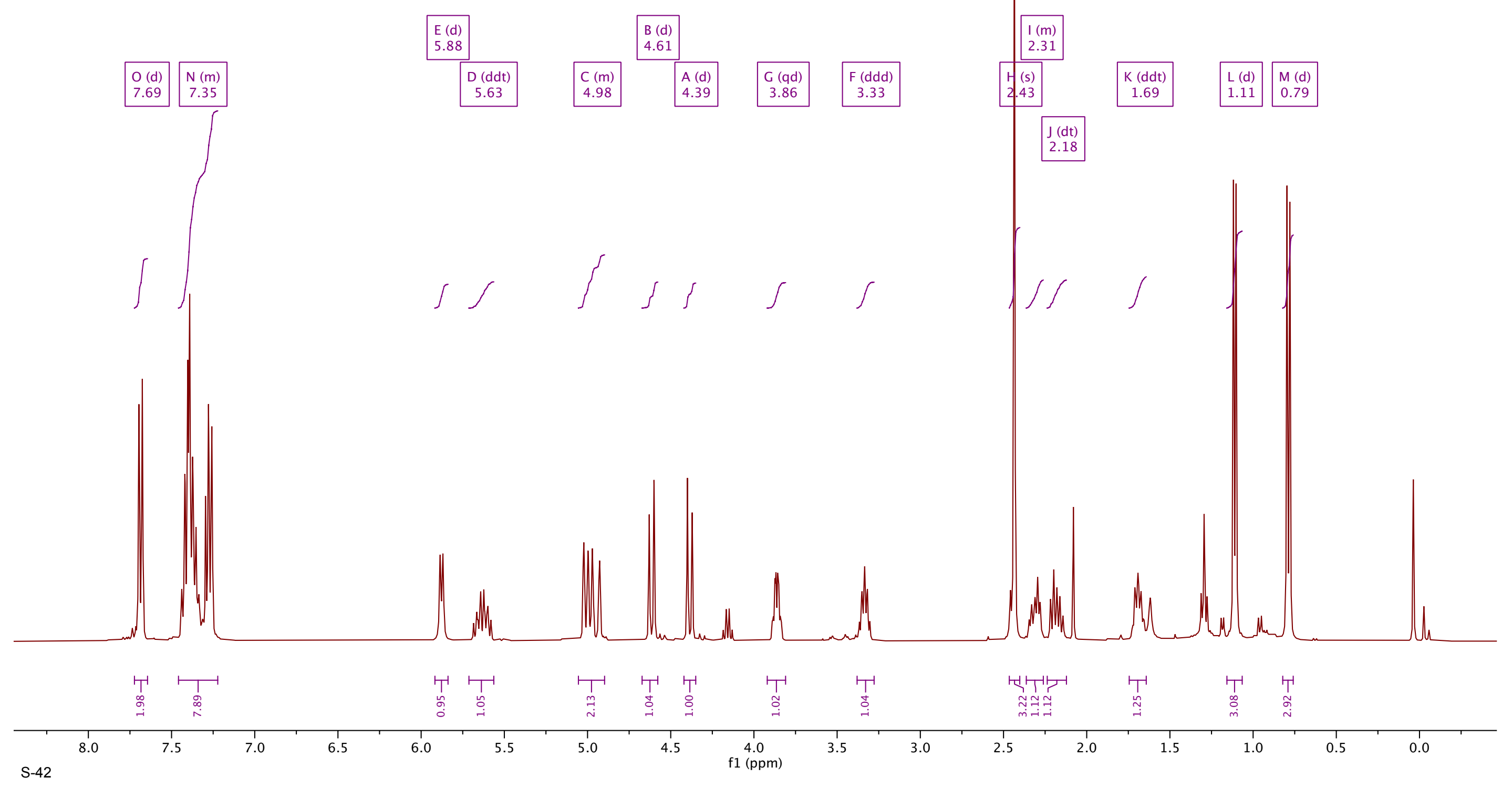




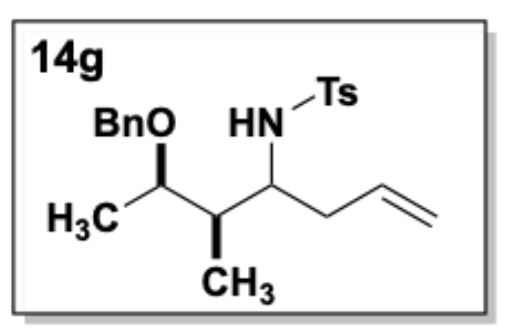

\begin{tabular}{rrrrrrrrrrrrrr}
1 \\
\hline $\mathrm{S}-43$
\end{tabular}




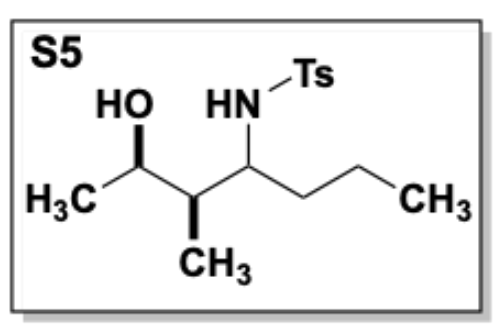

${ }^{13} \mathrm{C}\{1 \mathrm{H}\}$ NMR $\left(151 \mathrm{MHz}, \mathrm{cdcl}_{3}\right)$

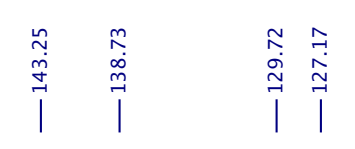

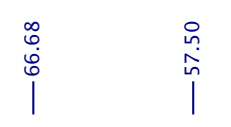

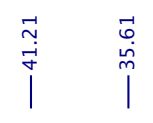

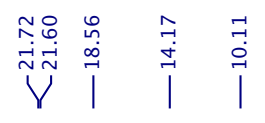

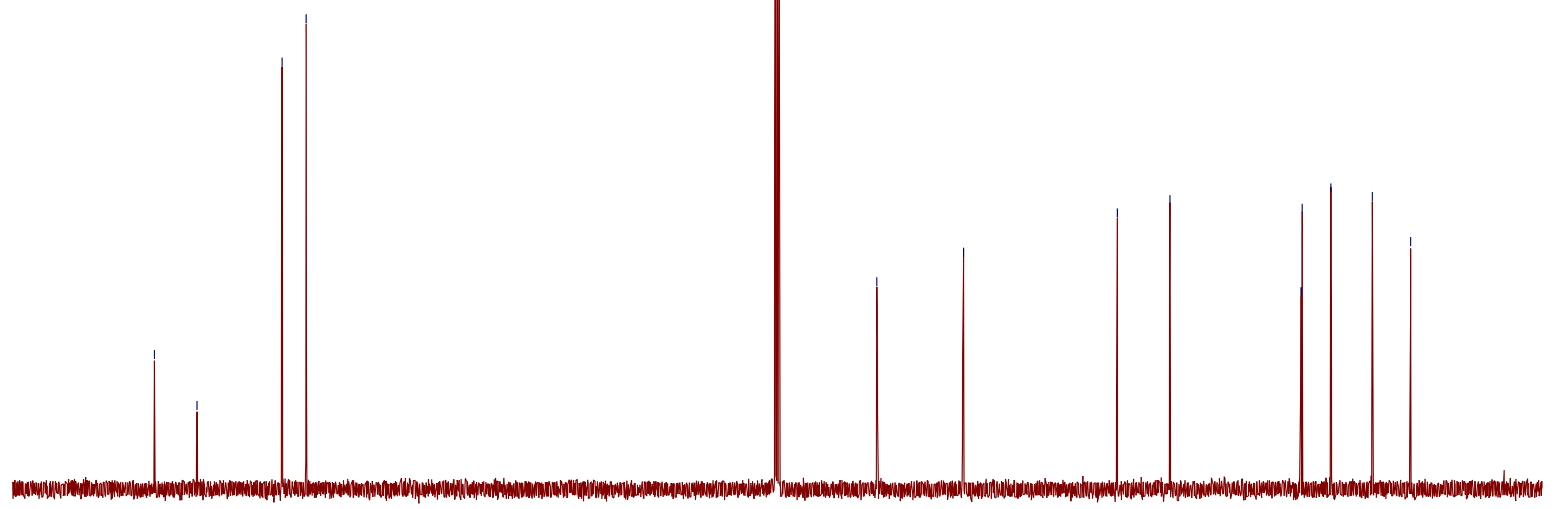

\begin{tabular}{|c|c|c|c|c|c|c|c|c|c|c|c|c|c|c|c|}
\hline 150 & $\begin{array}{l}1 \\
140\end{array}$ & $\begin{array}{l}1 \\
130\end{array}$ & 120 & 110 & 100 & 90 & $\begin{array}{l}80 \\
\mathrm{f} 1(\mathrm{ppm})\end{array}$ & 70 & 60 & 50 & 40 & 30 & 20 & 10 & $\begin{array}{l}1 \\
0\end{array}$ \\
\hline
\end{tabular}



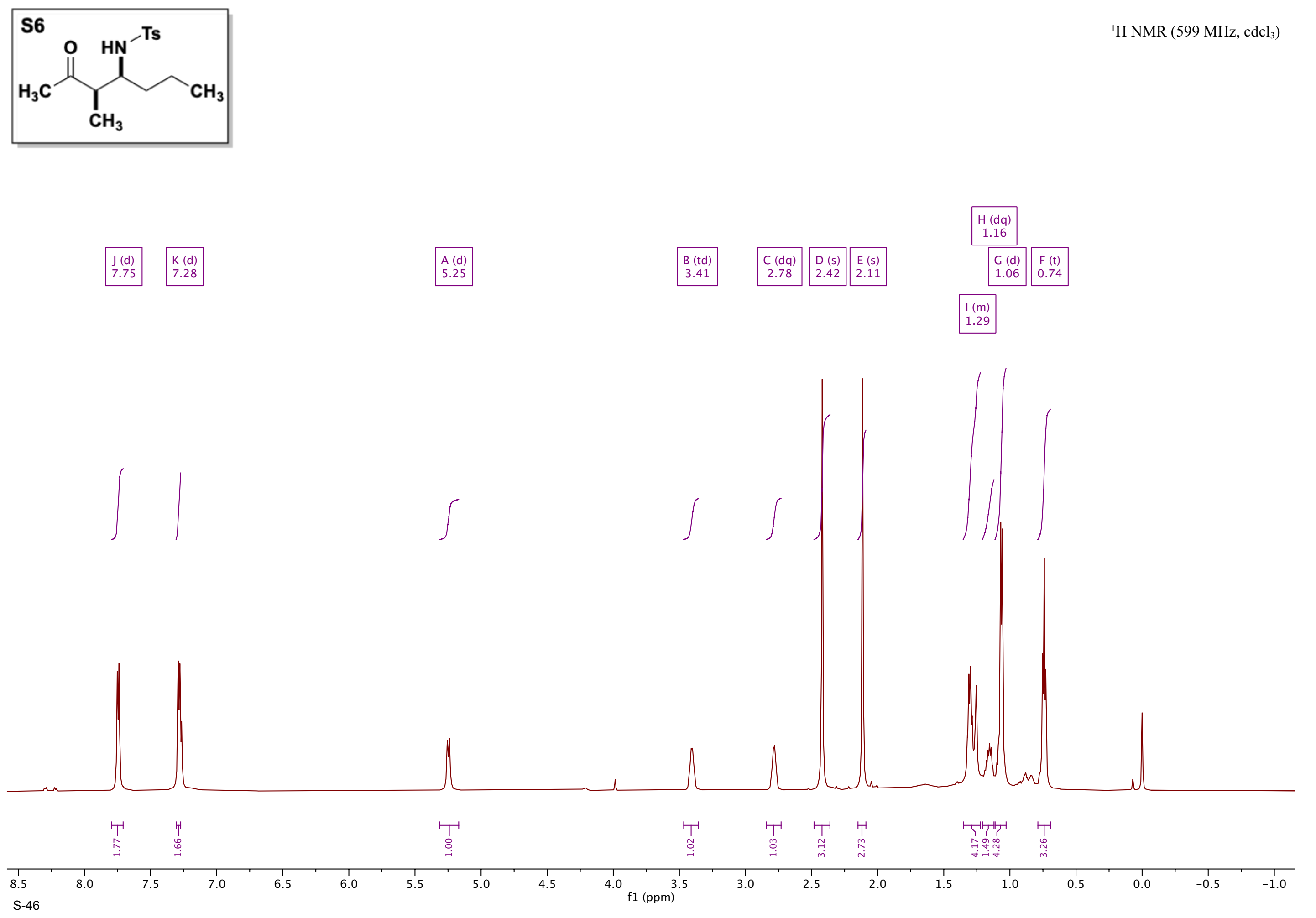


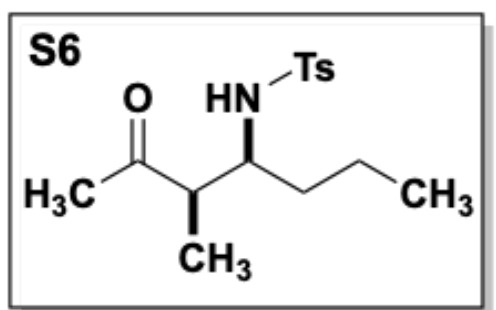

$\stackrel{\sim}{\sim}$

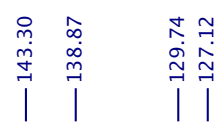

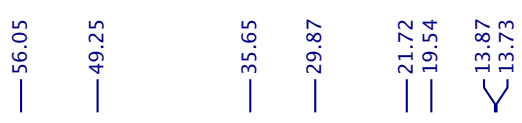

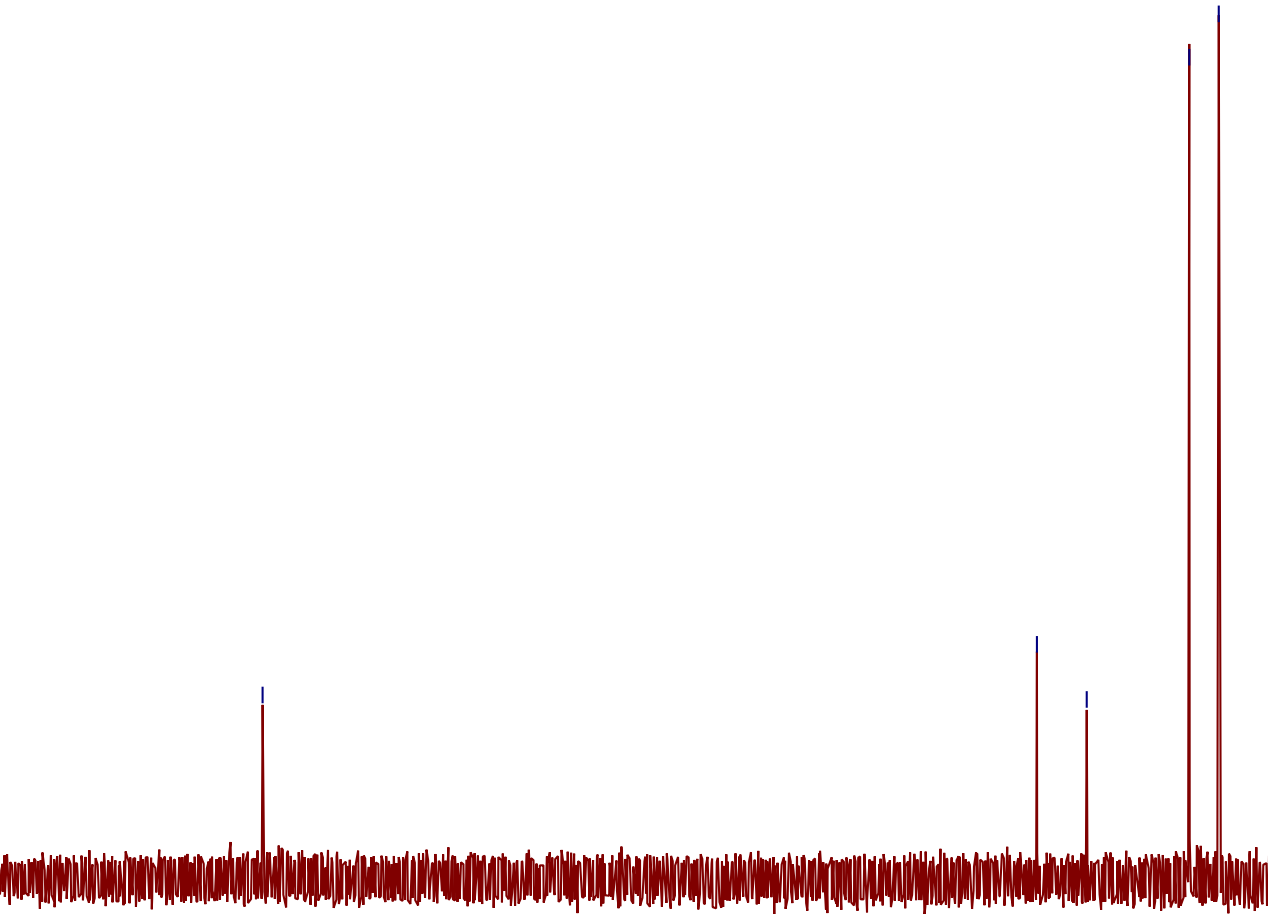

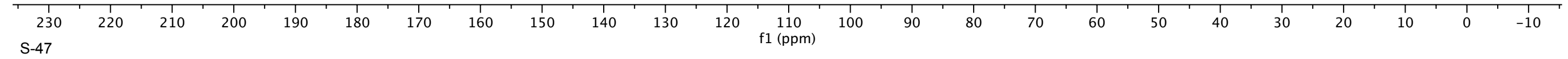




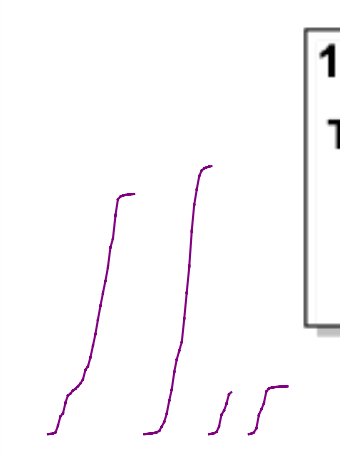

14h

TBDPSO $\mathrm{HN}^{-\mathrm{Ts}}$

$\mathrm{H}_{3} \mathrm{C}$
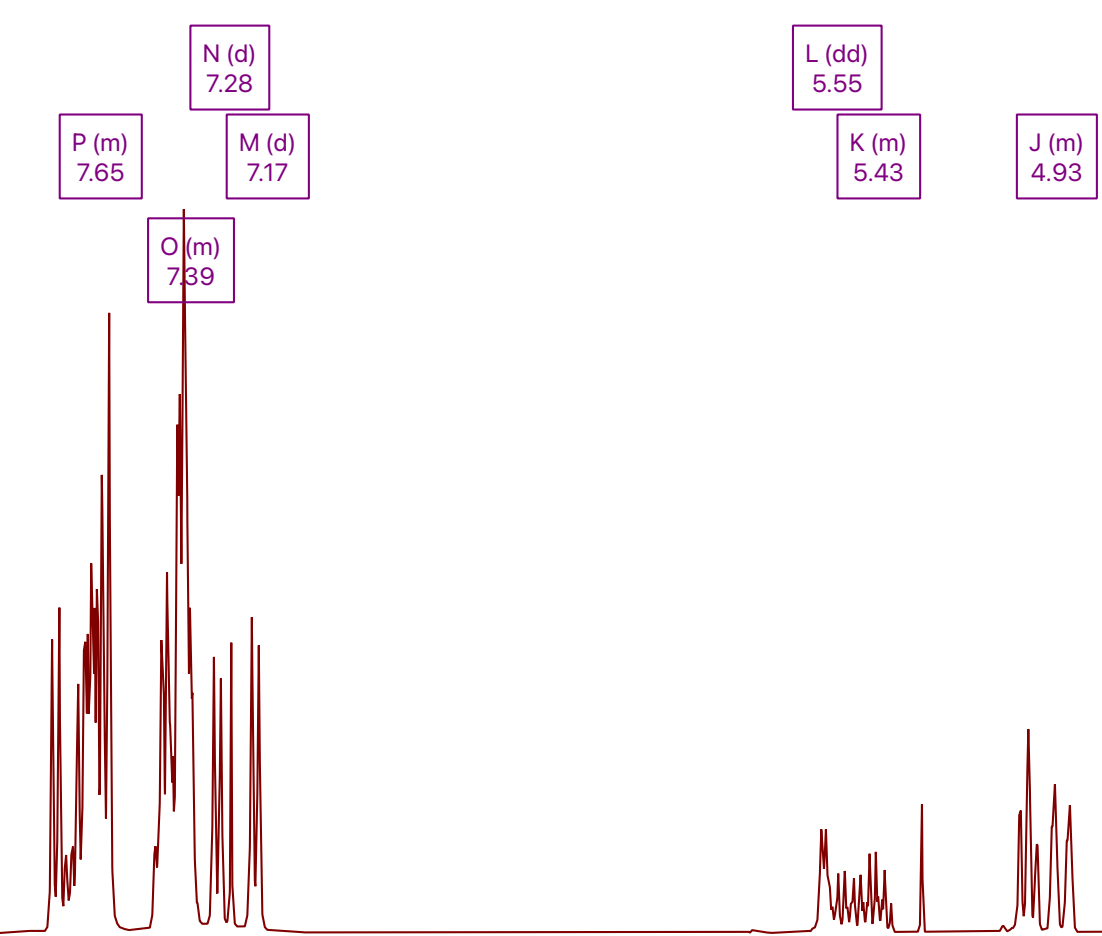

MU M
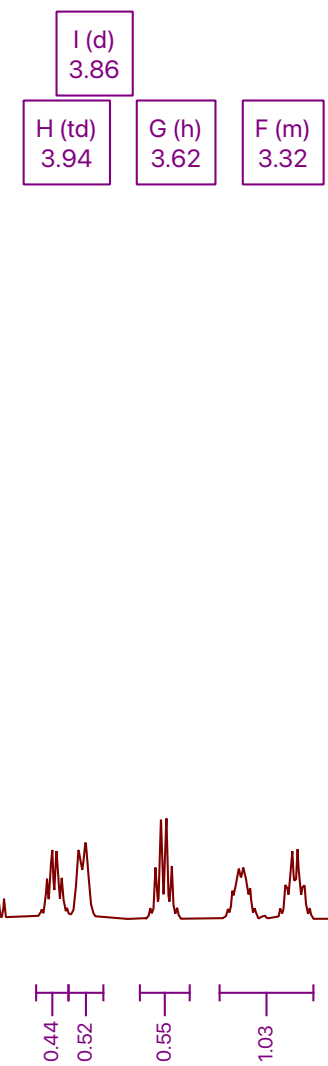

f1 (ppm)
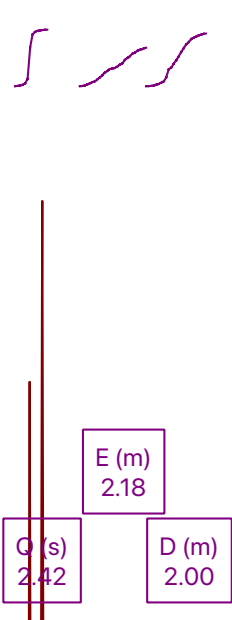

C (m) 1.49

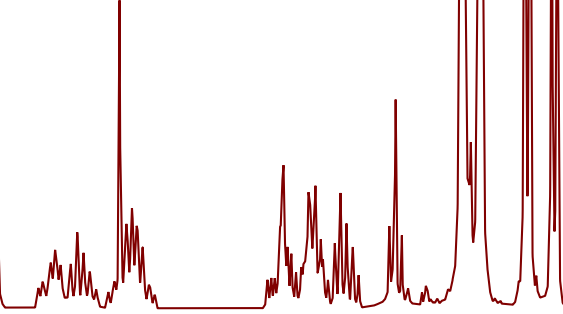




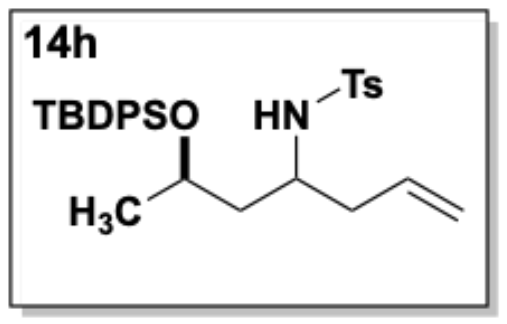

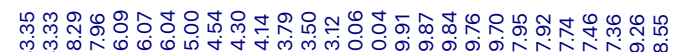

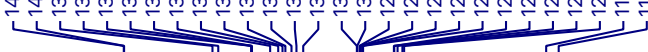

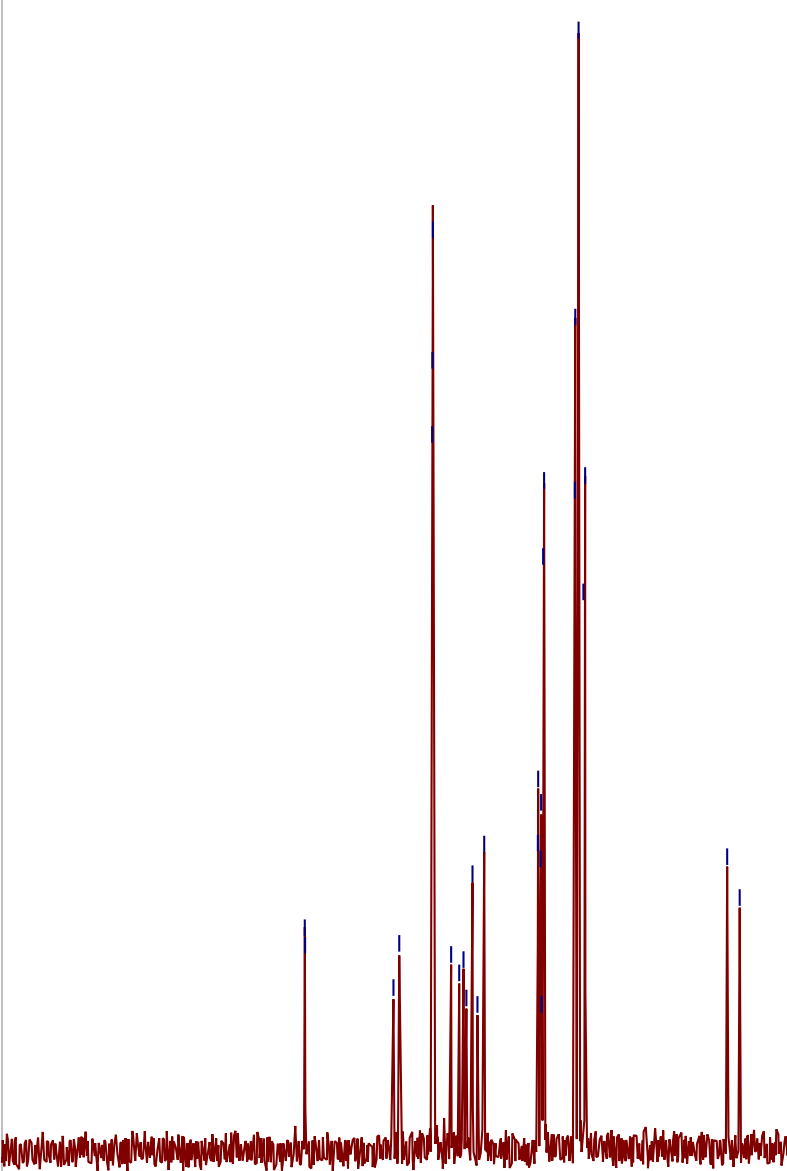

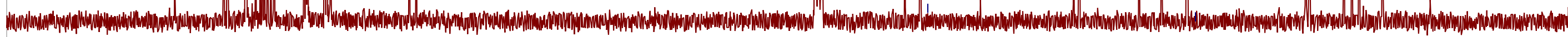

${ }^{13} \mathrm{C}\{1 \mathrm{H}\} \mathrm{NMR}\left(101 \mathrm{MHz}, \mathrm{CDCl}_{3}\right)$

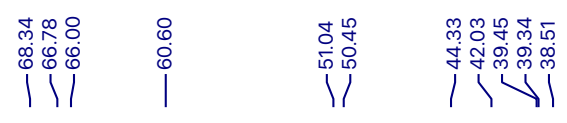

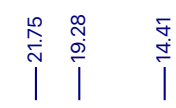$$
60
$$

150

140

130

120

110

100

90

f1 (ppm)
70

60

50

40

30

20

10 


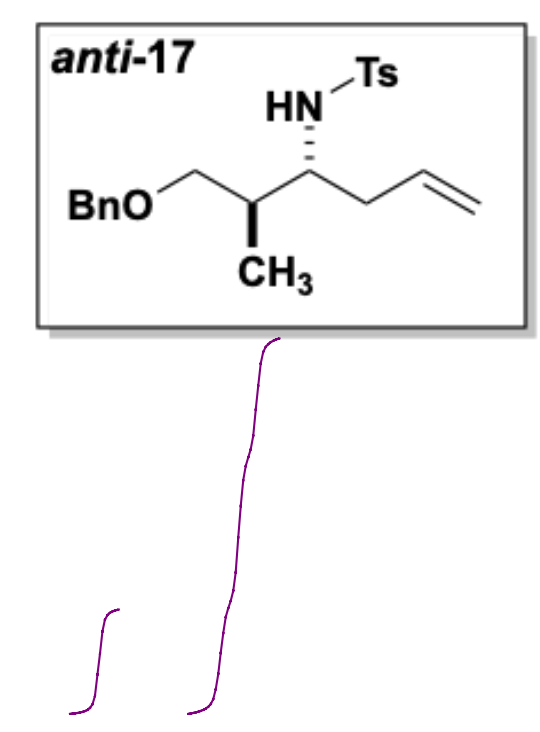

${ }^{1} \mathrm{H}$ NMR (599 MHz, Chloroform- $d$ )
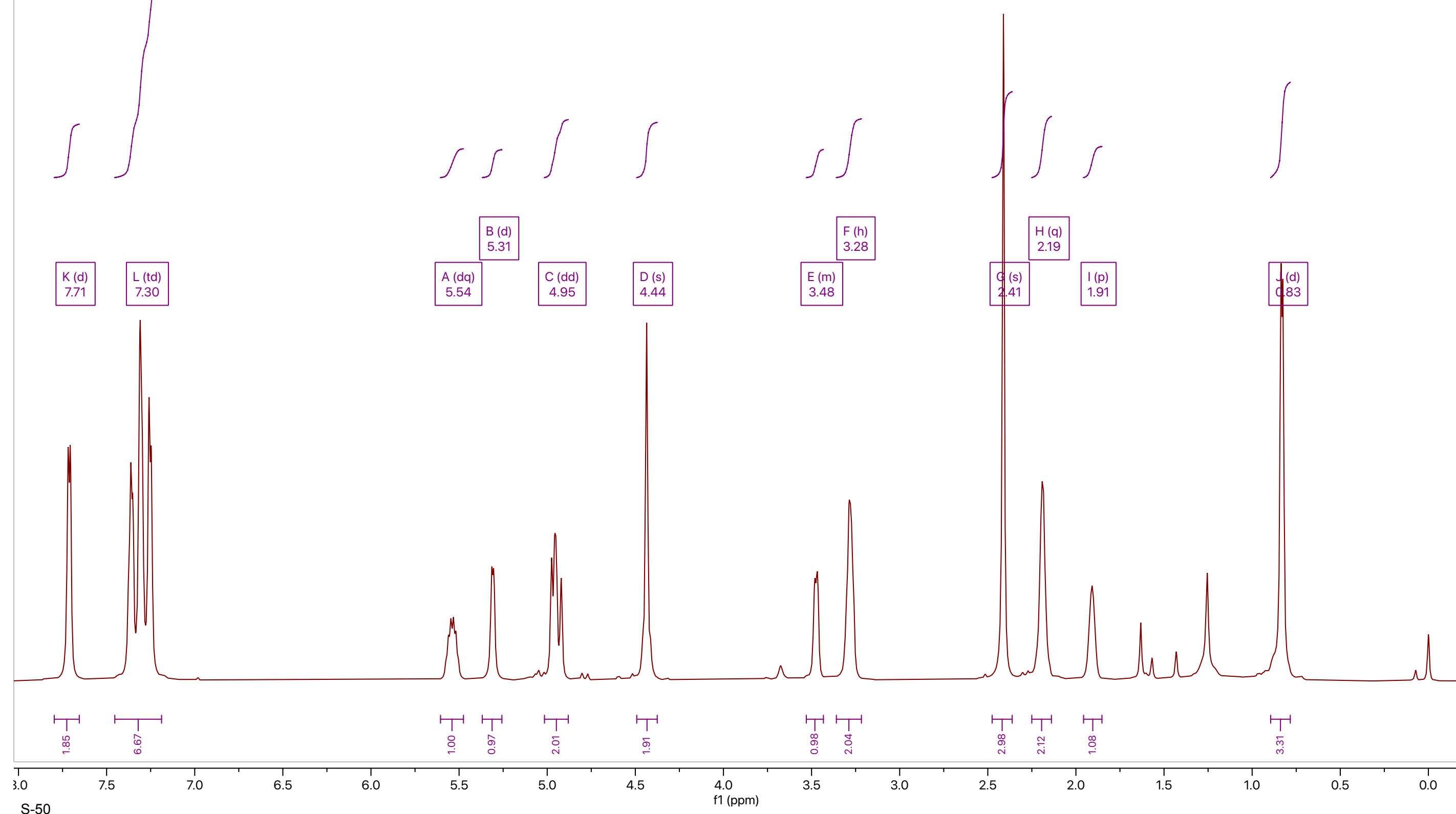

S-50

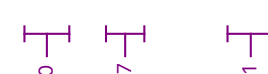

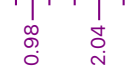

$\prod_{\infty}^{\infty} T^{2}$

† 


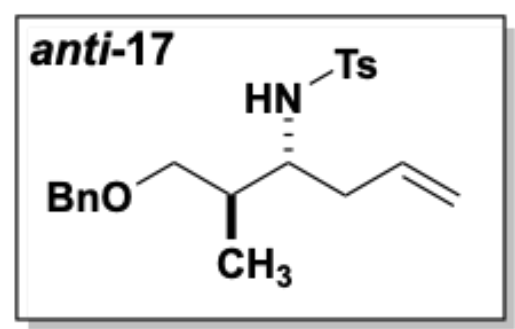

${ }^{13} \mathrm{C}\{1 \mathrm{H}\}$ NMR $\left(151 \mathrm{MHz}, \mathrm{cdcl}_{3}\right)$

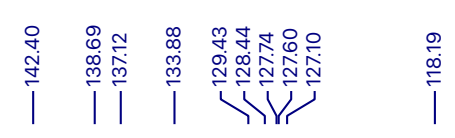

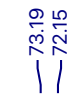

剚高

密
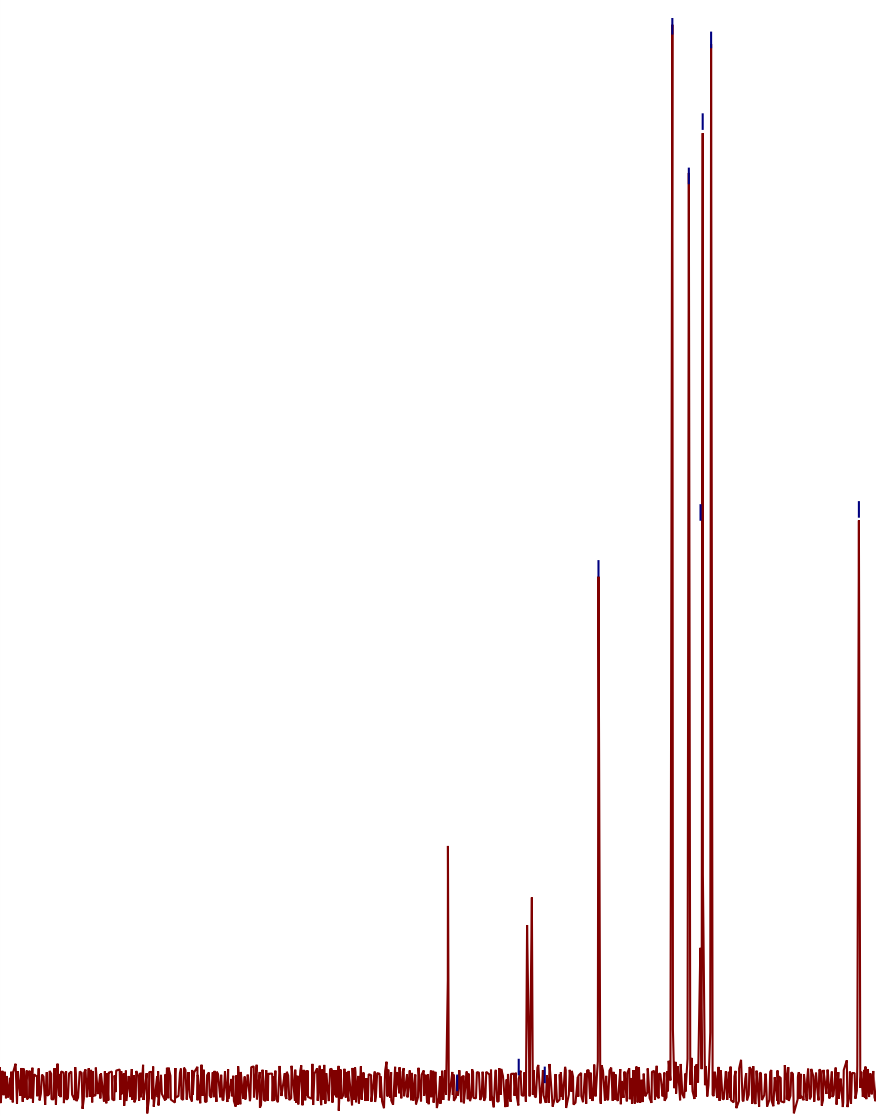

|

11

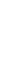



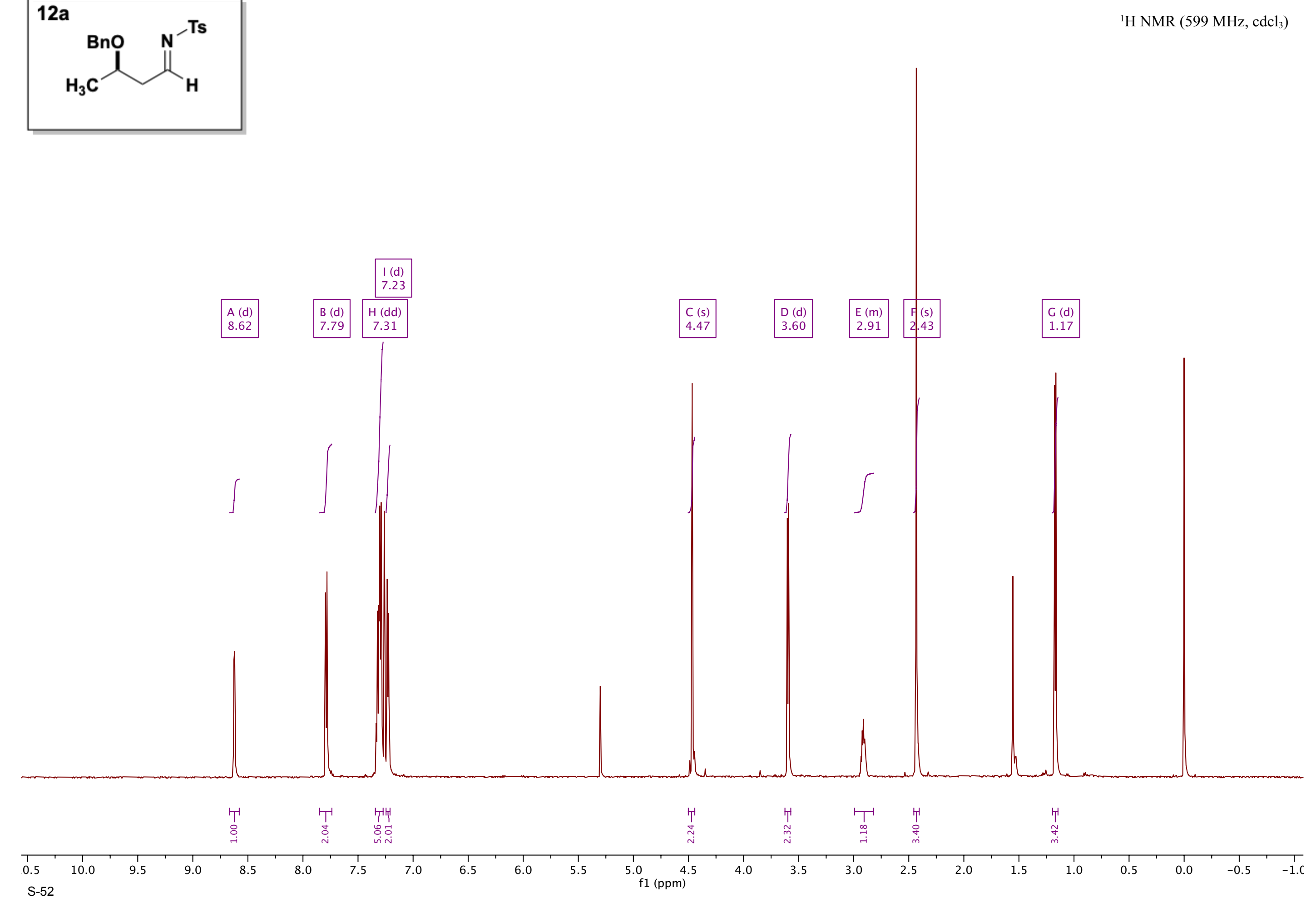

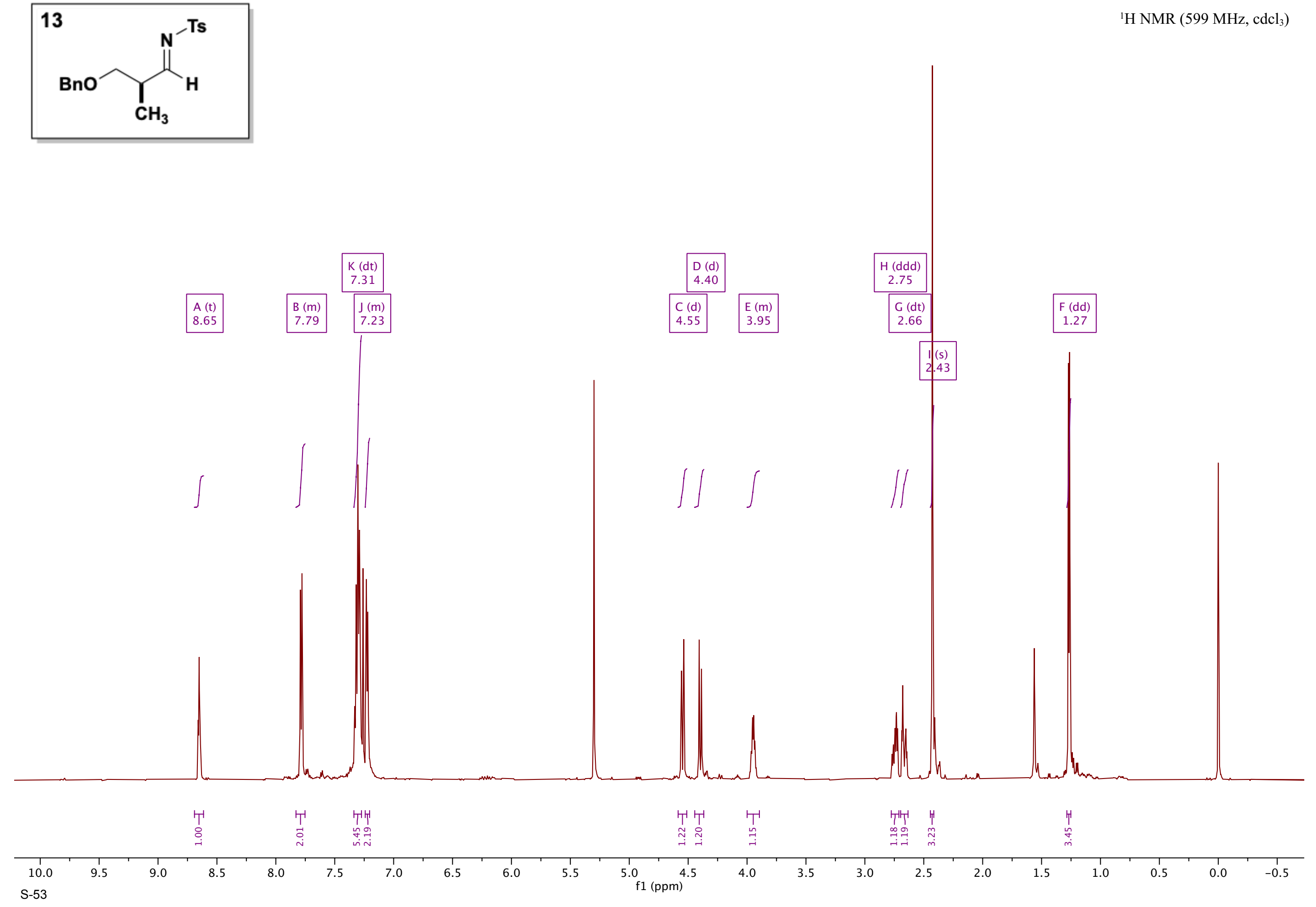

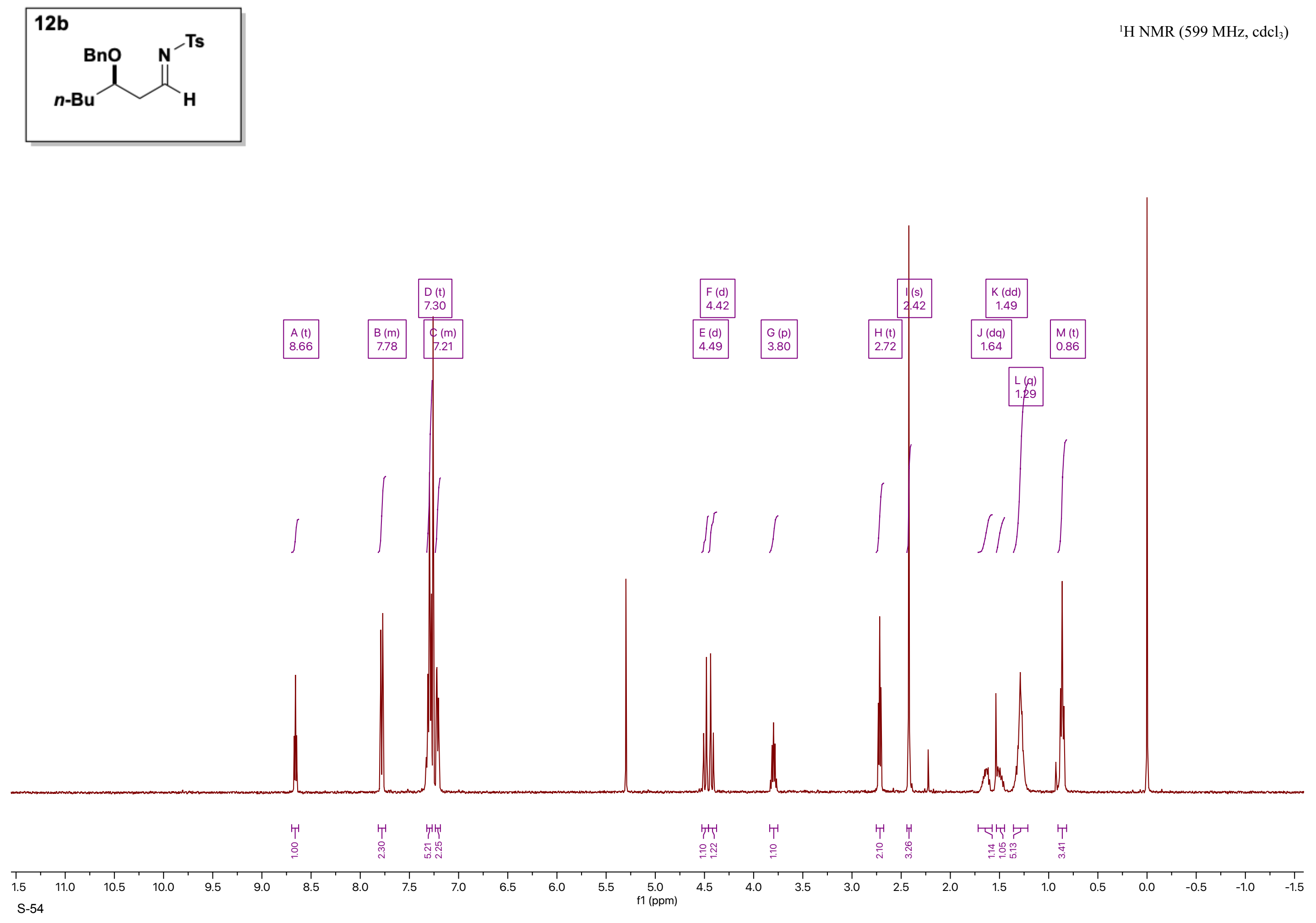

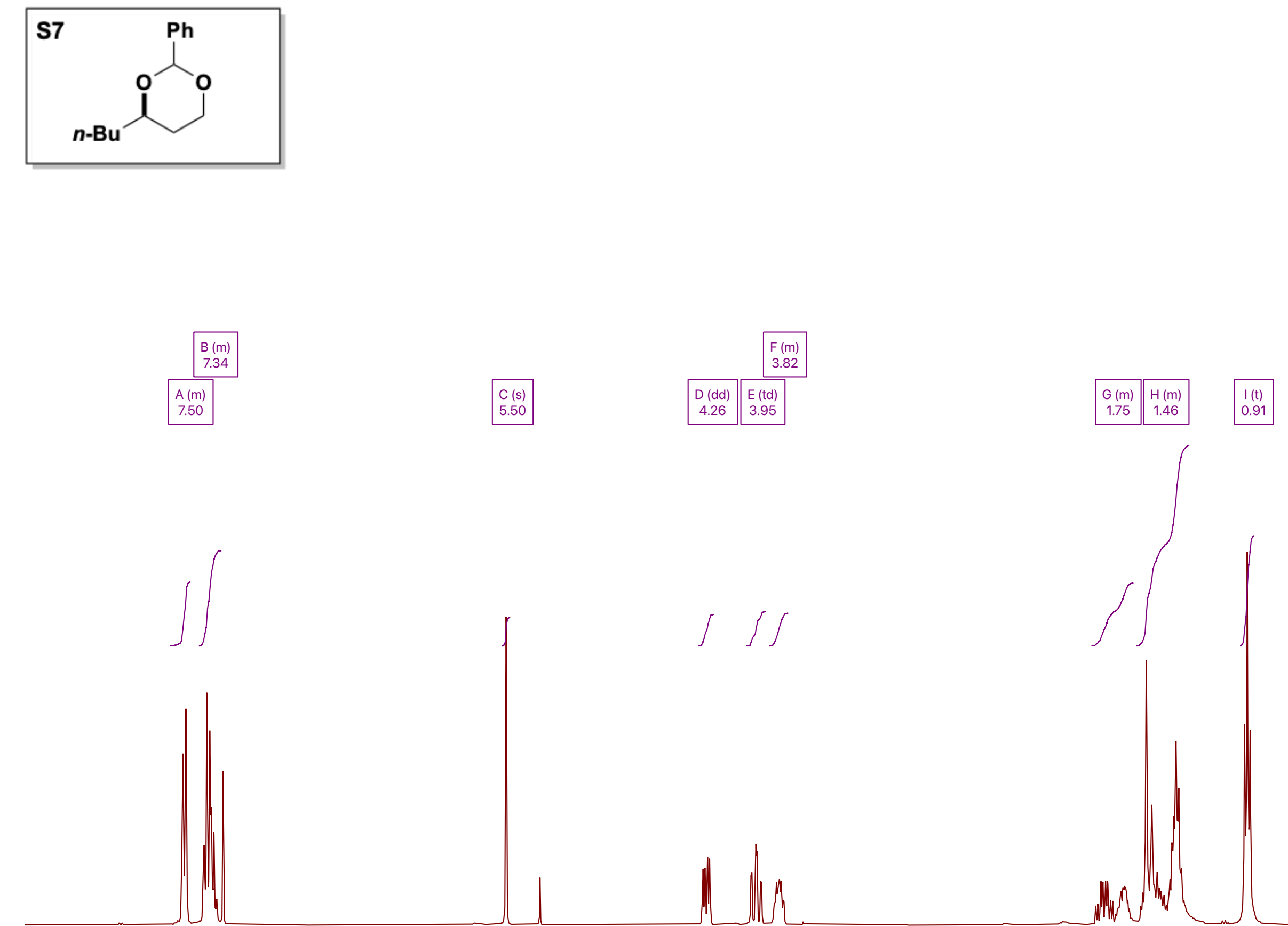

\begin{tabular}{lll}
4.26 & 3.95 \\
\hline
\end{tabular}

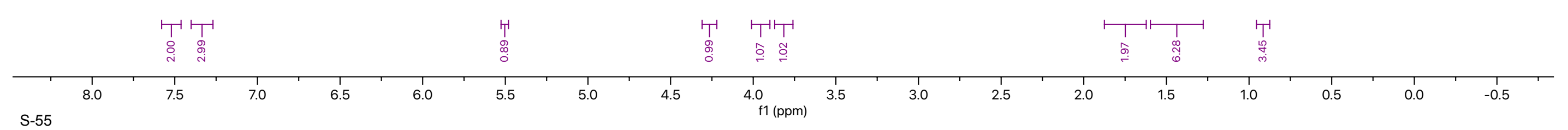




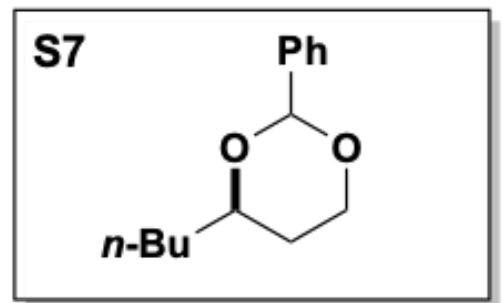

${ }^{13} \mathrm{C}\{\mathrm{H}\}$ NMR (101 MHz, Acetone)

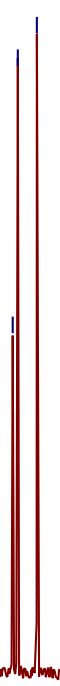

$$
\text { S-5 }
$$

S-56 


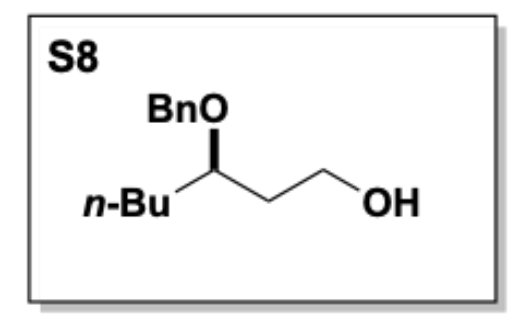

${ }^{1} \mathrm{H}$ NMR (400 MHz, $\mathrm{CDCl}_{3}$ )

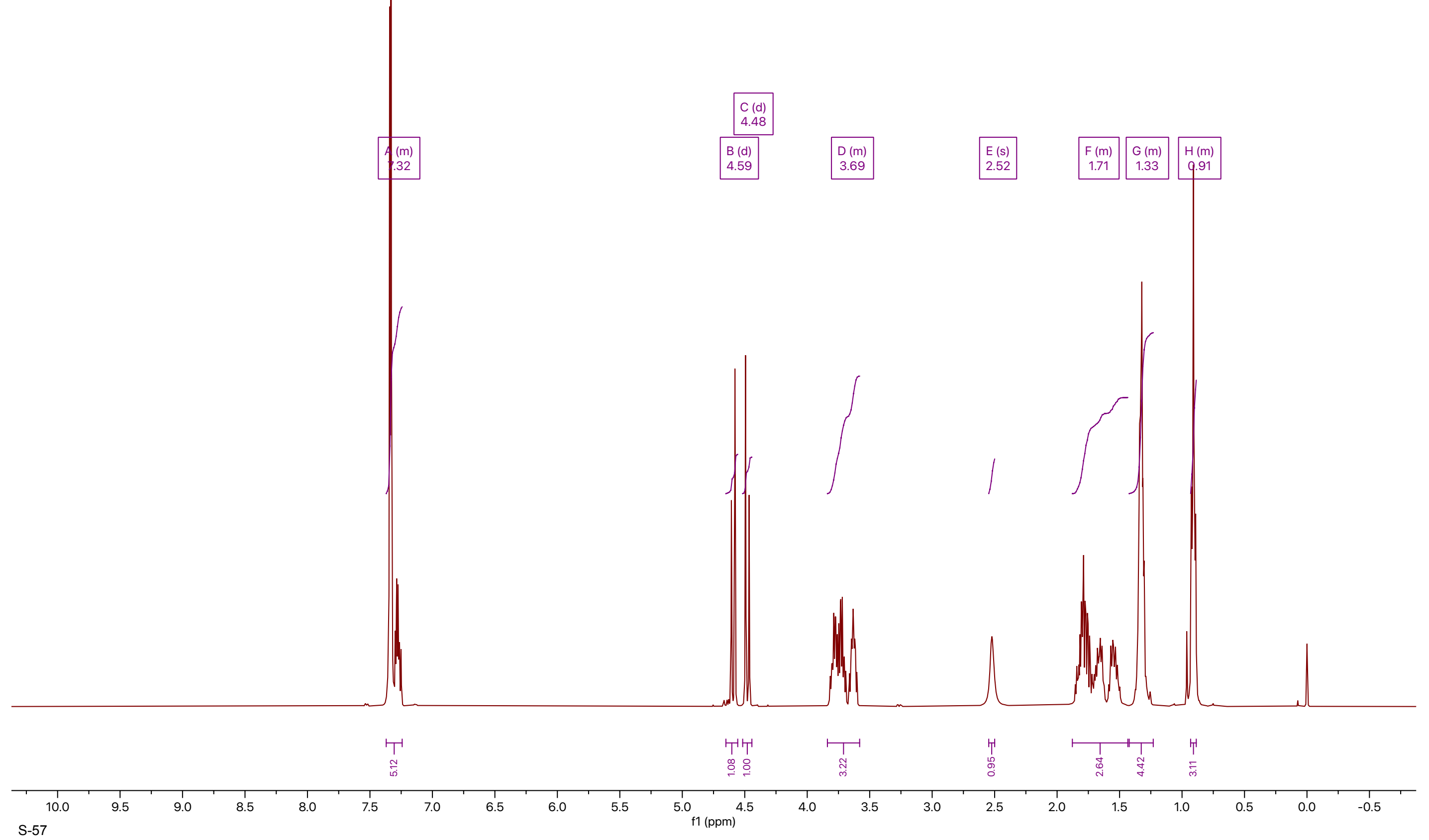




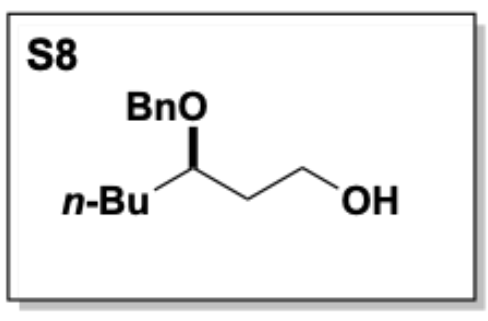

${ }^{13} \mathrm{C}\{\mathrm{H}\}$ NMR (101 MHz, $\left.\mathrm{CDCl}_{3}\right)$

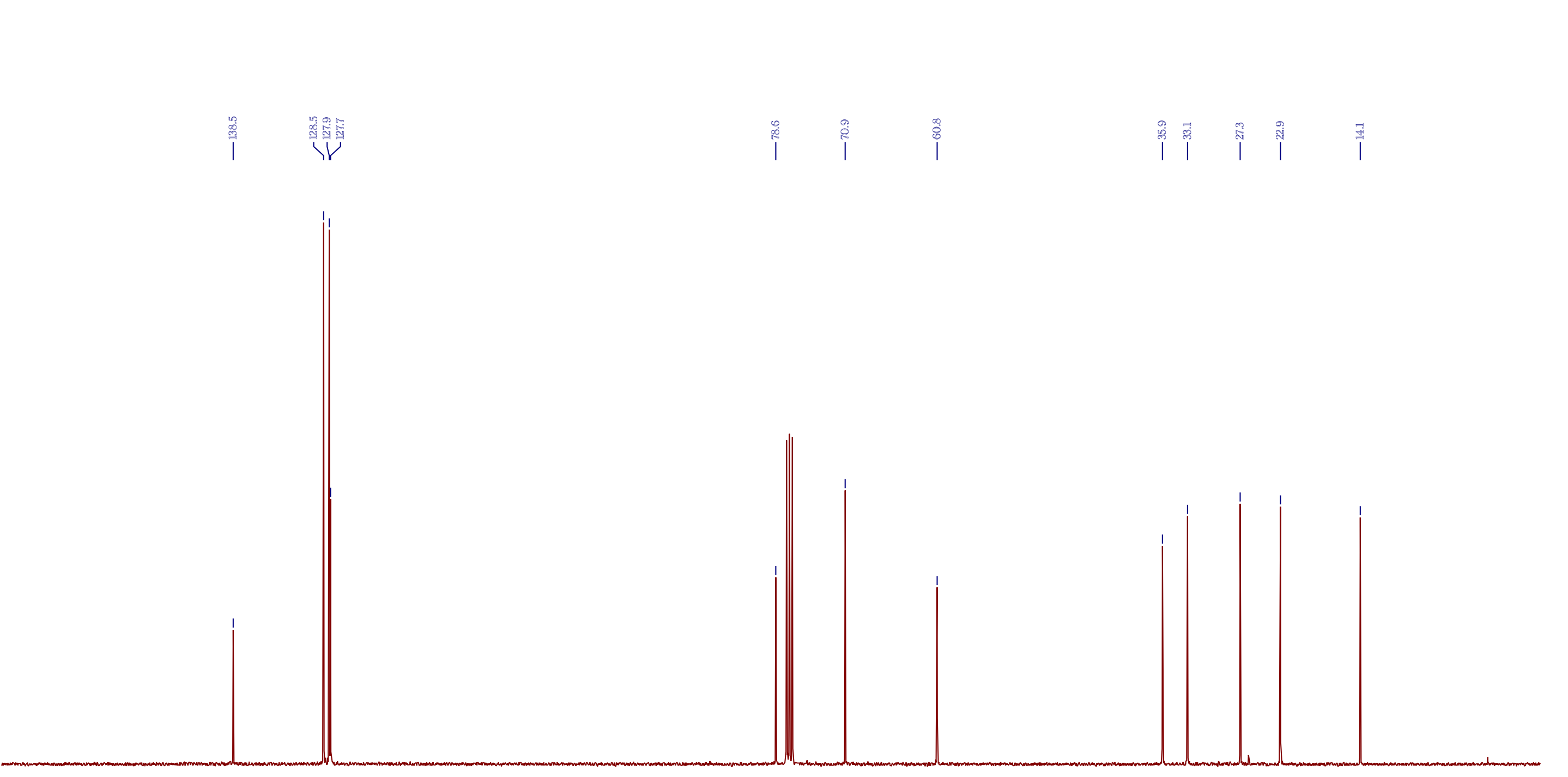

\begin{tabular}{|c|c|c|c|c|c|c|c|c|c|c|c|c|c|c|c|c|}
\hline $\begin{array}{r}1 \\
160 \\
S-58\end{array}$ & 150 & 140 & 130 & 120 & 110 & 100 & $\begin{array}{l}1 \\
90\end{array}$ & $\begin{array}{c}1 \\
80 \\
\mathrm{f} 1(\mathrm{ppm})\end{array}$ & 70 & 60 & $\begin{array}{l}1 \\
50\end{array}$ & 40 & 30 & 20 & 10 & $\begin{array}{l}1 \\
0\end{array}$ \\
\hline
\end{tabular}




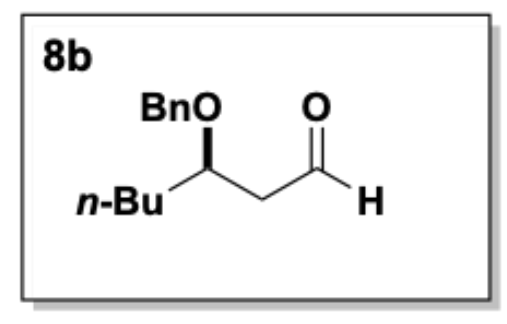

${ }^{1} \mathrm{H}$ NMR (400 MHz, $\mathrm{CDCl}_{3}$ )

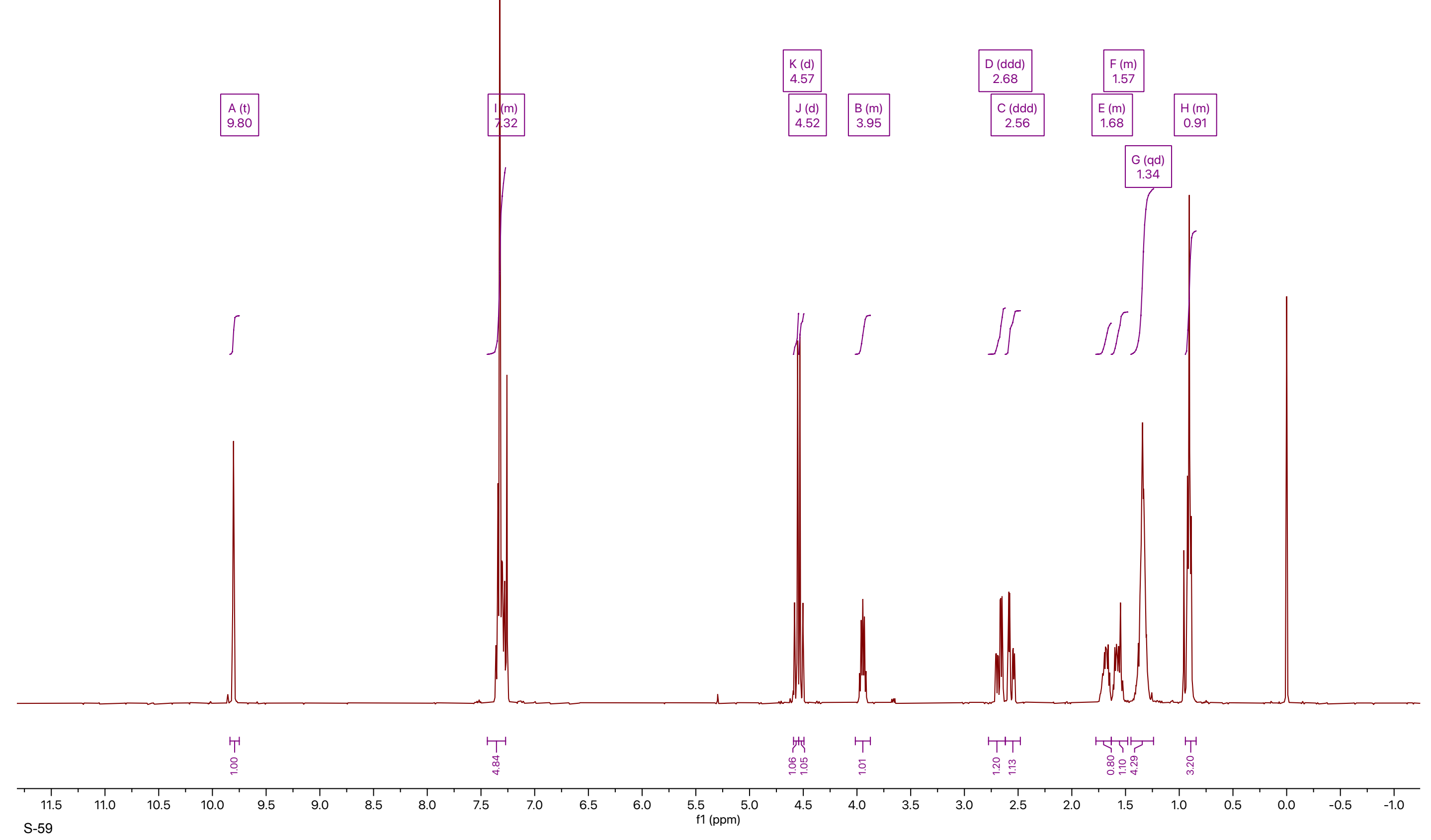



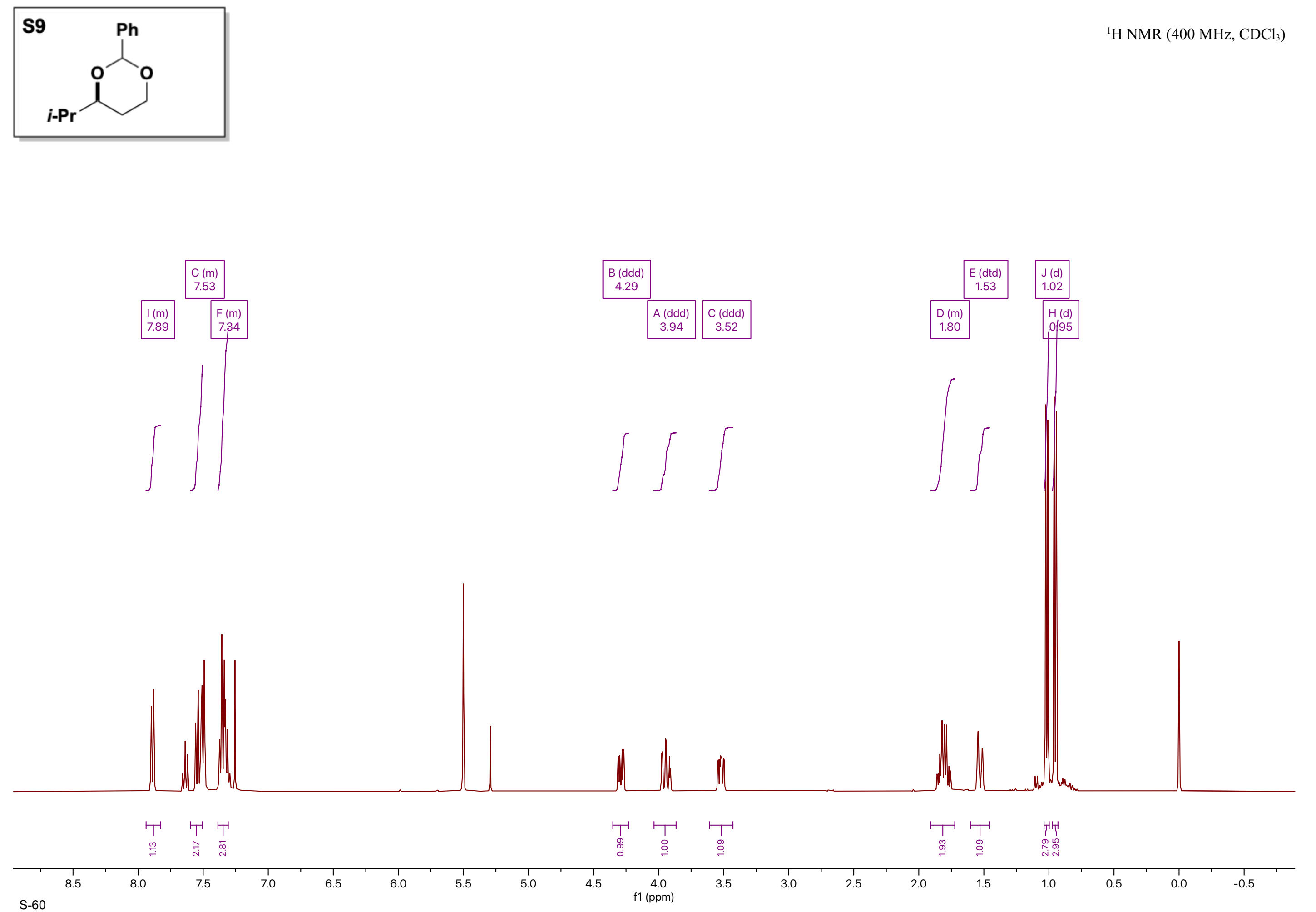

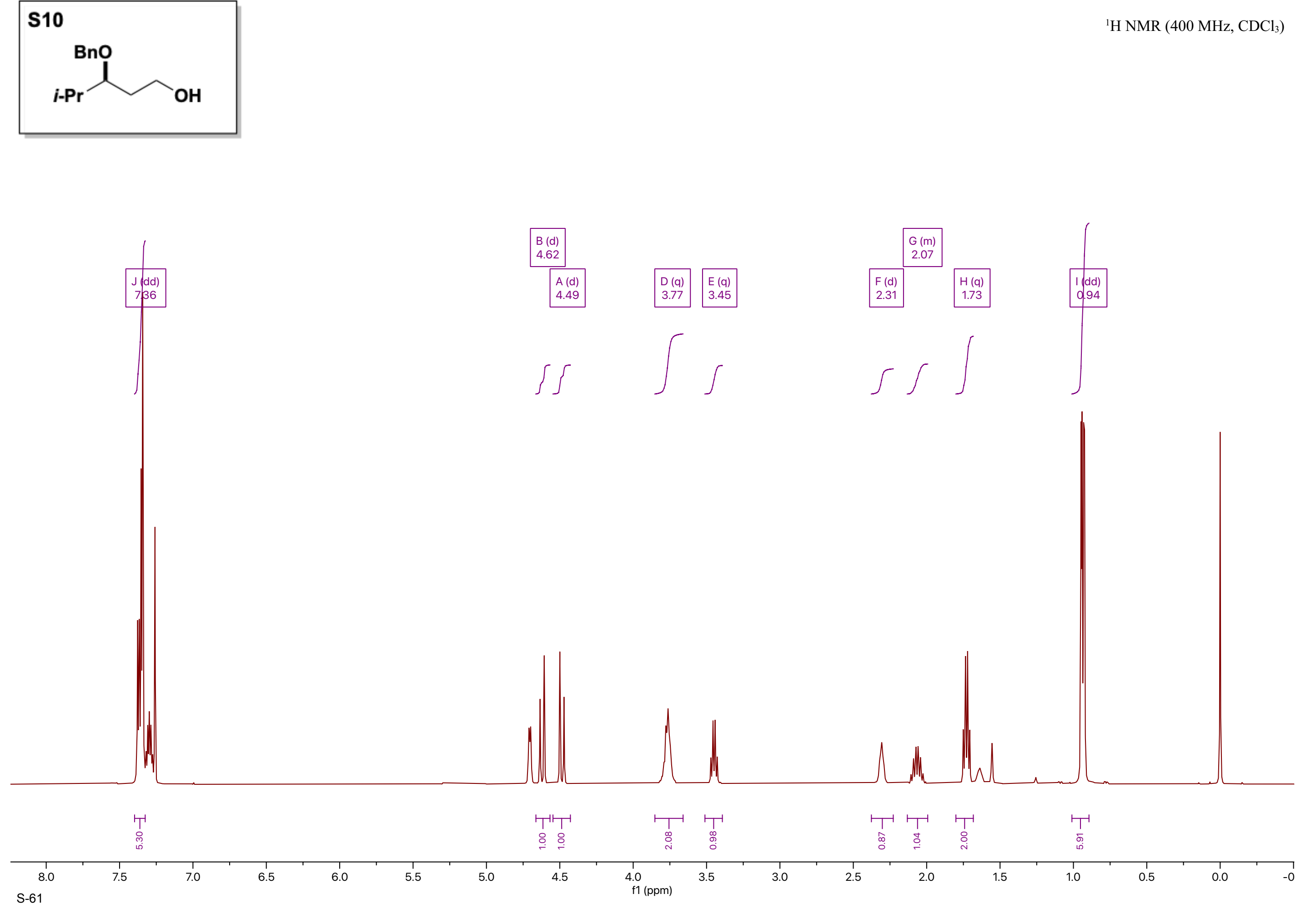


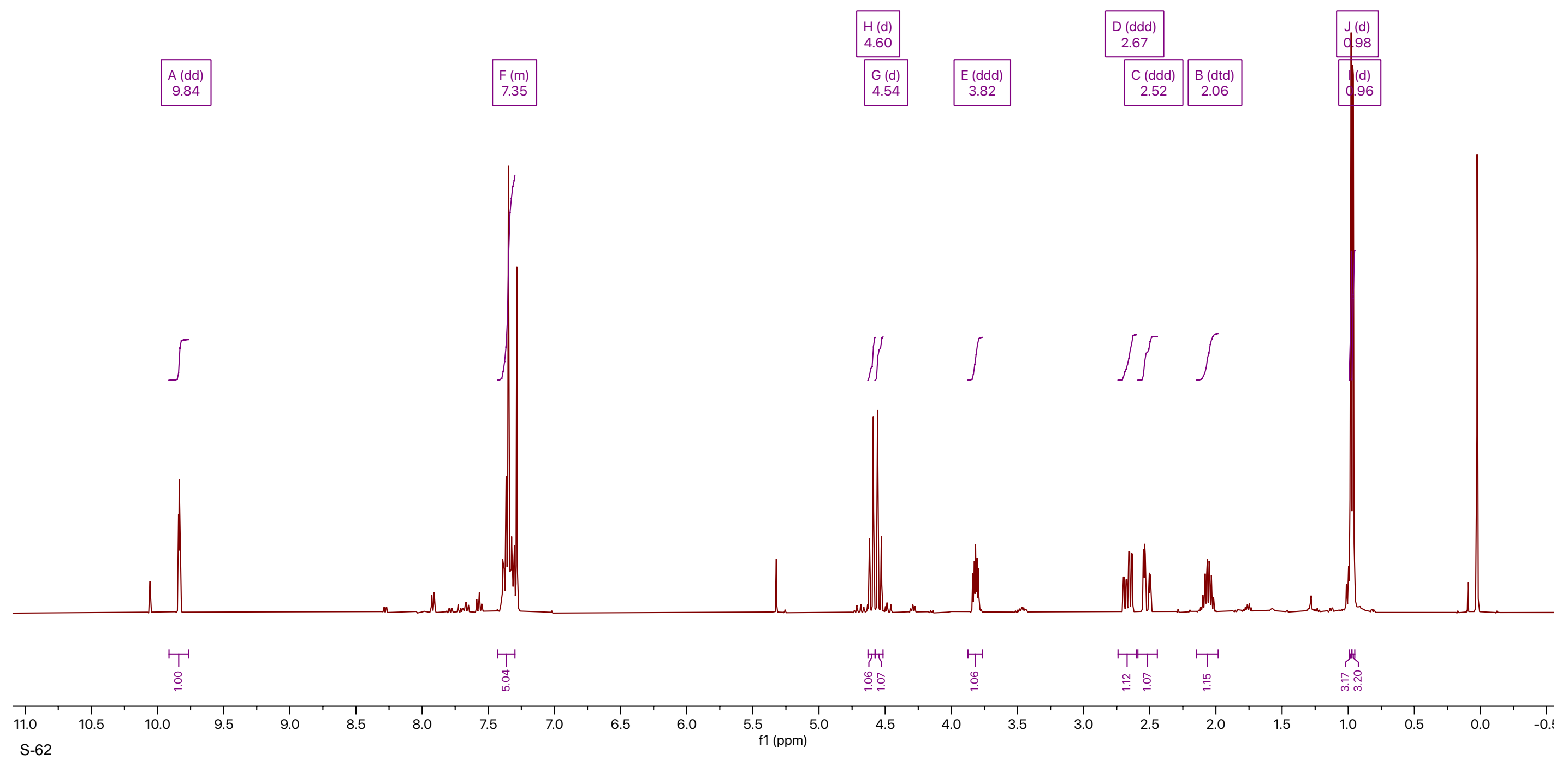




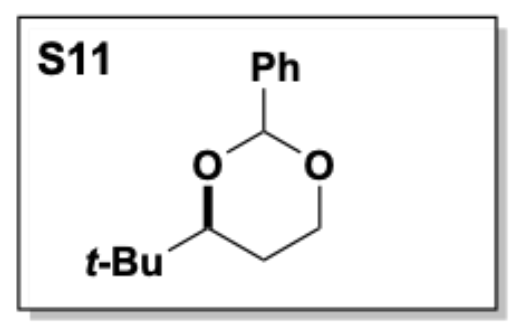

${ }^{13} \mathrm{C}\{\mathrm{H}\}$ NMR (101 MHz, $\left.\mathrm{CDCl}_{3}\right)$
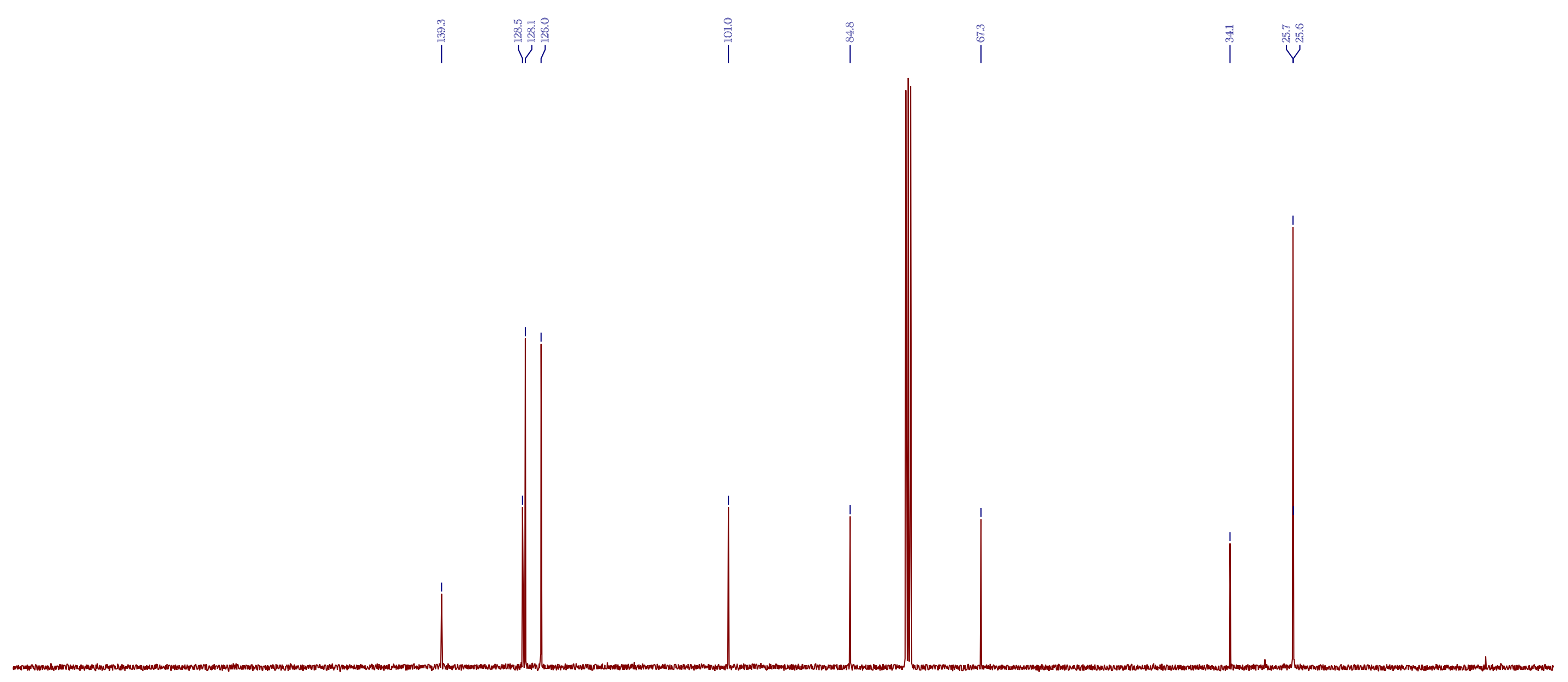

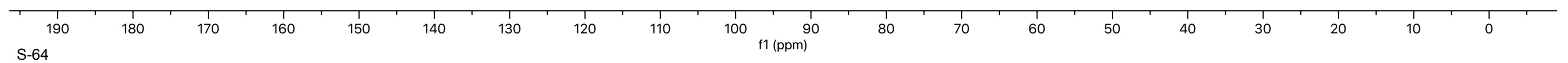


S12

$\overbrace{\mathrm{OH}}^{\mathrm{BnO}}$
${ }^{1} \mathrm{H}$ NIMR $\left(400 \mathrm{MHz}, \mathrm{CDCl}_{3}\right)$

\begin{tabular}{|c|c|}
\hline$B(m)$ & $C(m)$ \\
4.64 & $D(d d)$ \\
3.75 & 3.26 \\
\hline
\end{tabular}

$\int$
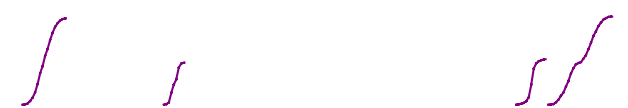

M

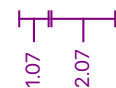
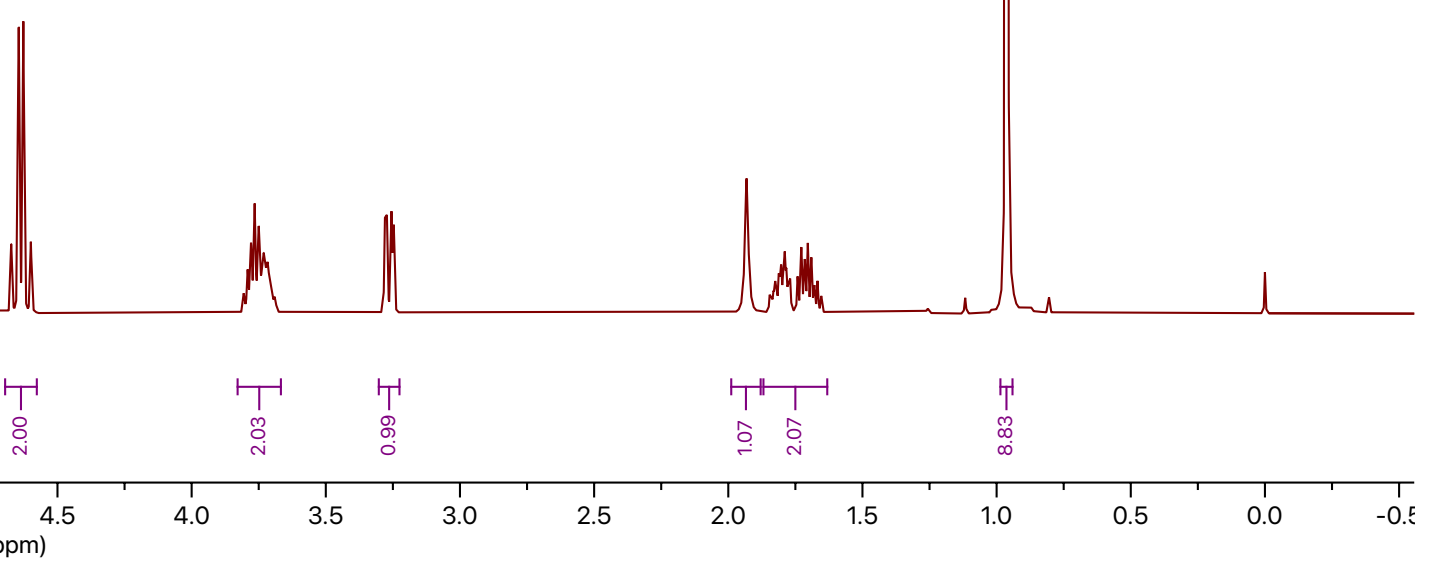


\section{S12 \\ Bno ${ }_{t-\mathrm{Bu}} \mathrm{OH}$}
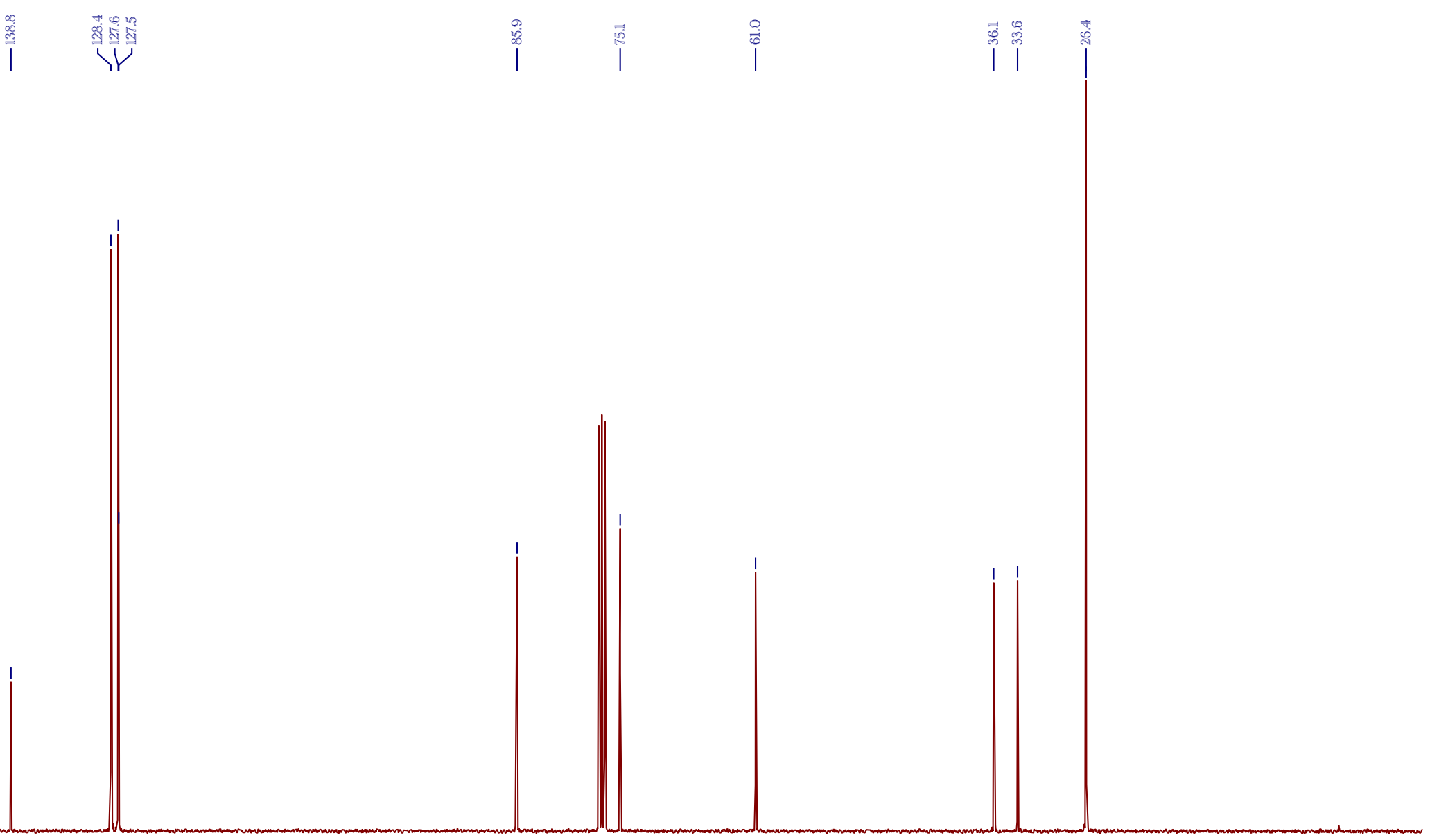

蒂

${ }^{3} \mathrm{C}\{\mathrm{H}\} \mathrm{NMR}\left(101 \mathrm{MHz}, \mathrm{CDCl}_{3}\right)$ 


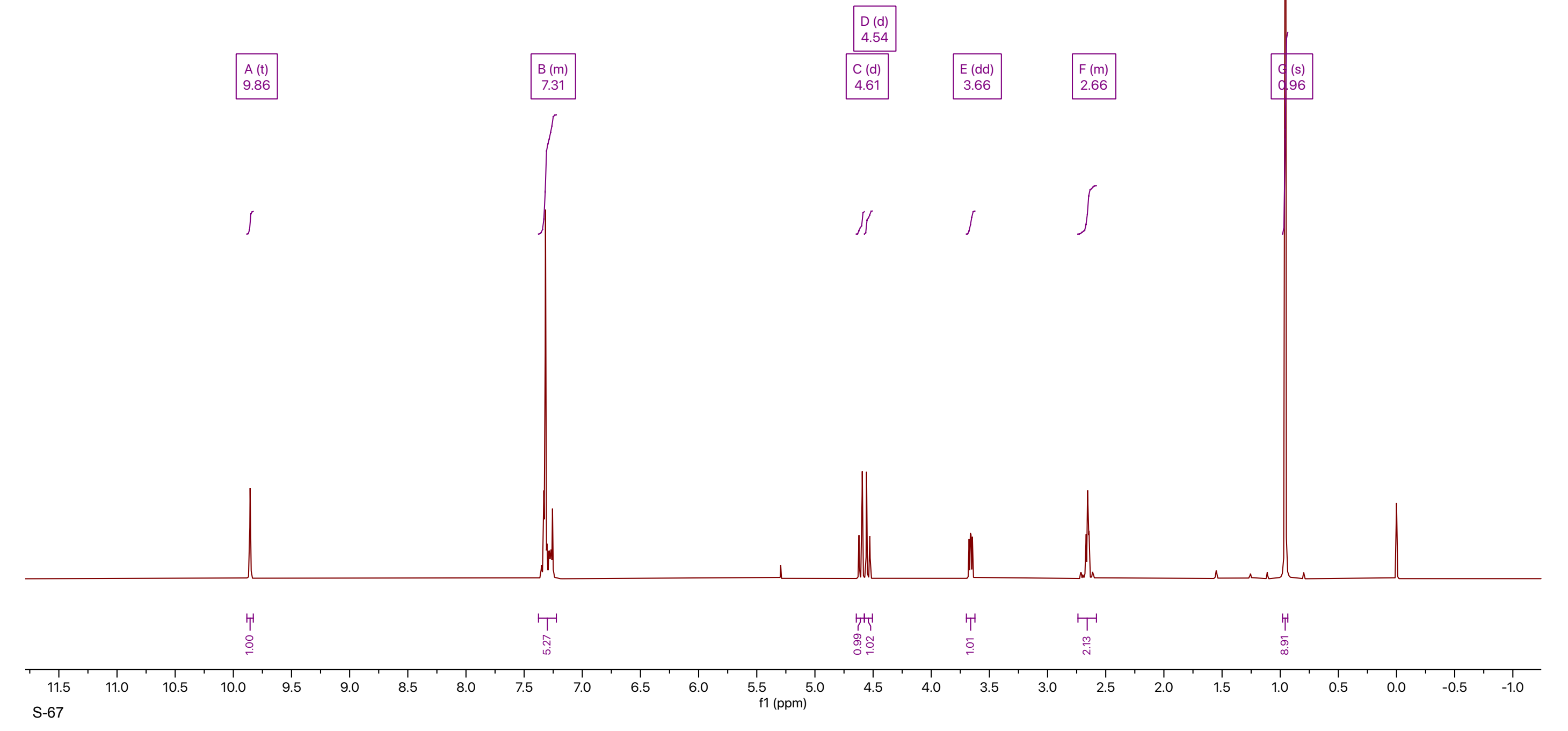



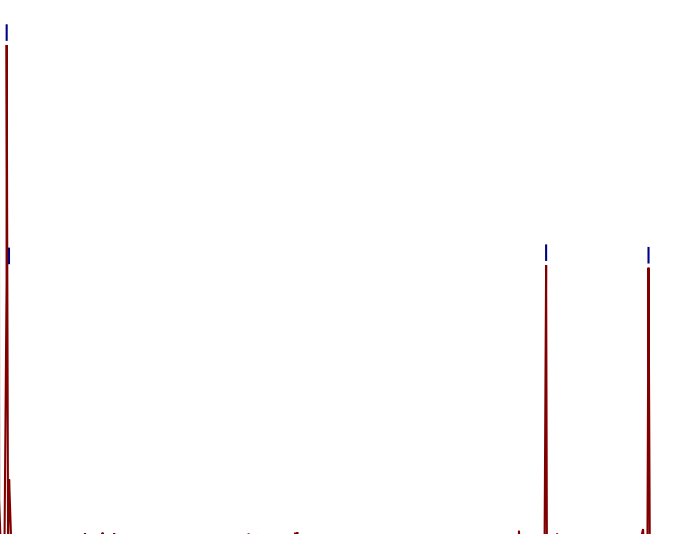


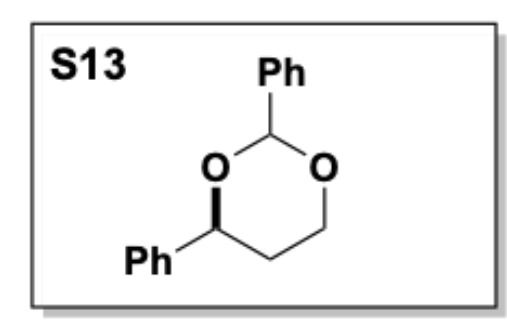

${ }^{1} \mathrm{H}$ NMR (400 MHz, $\mathrm{CDCl}_{3}$ )

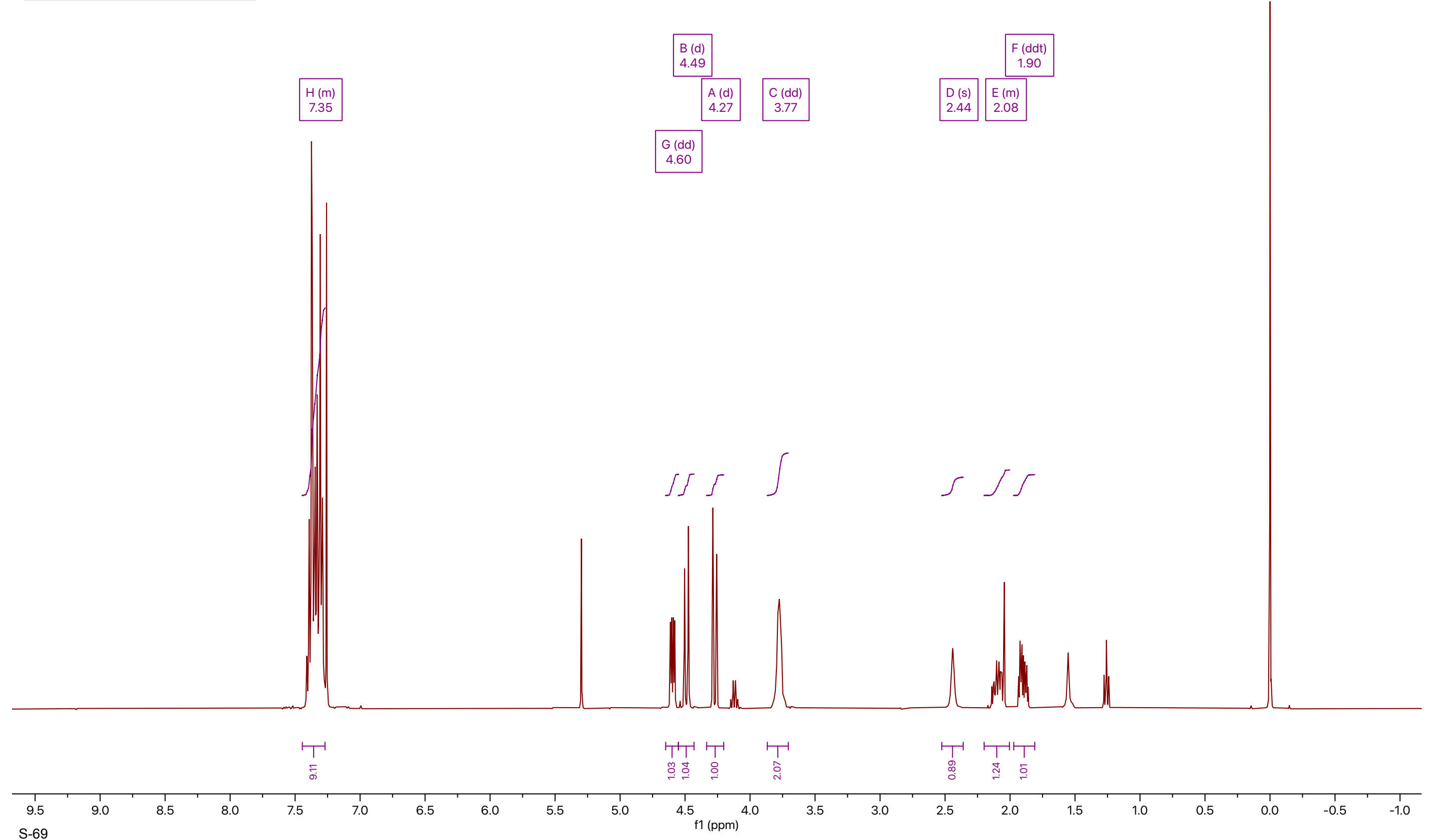




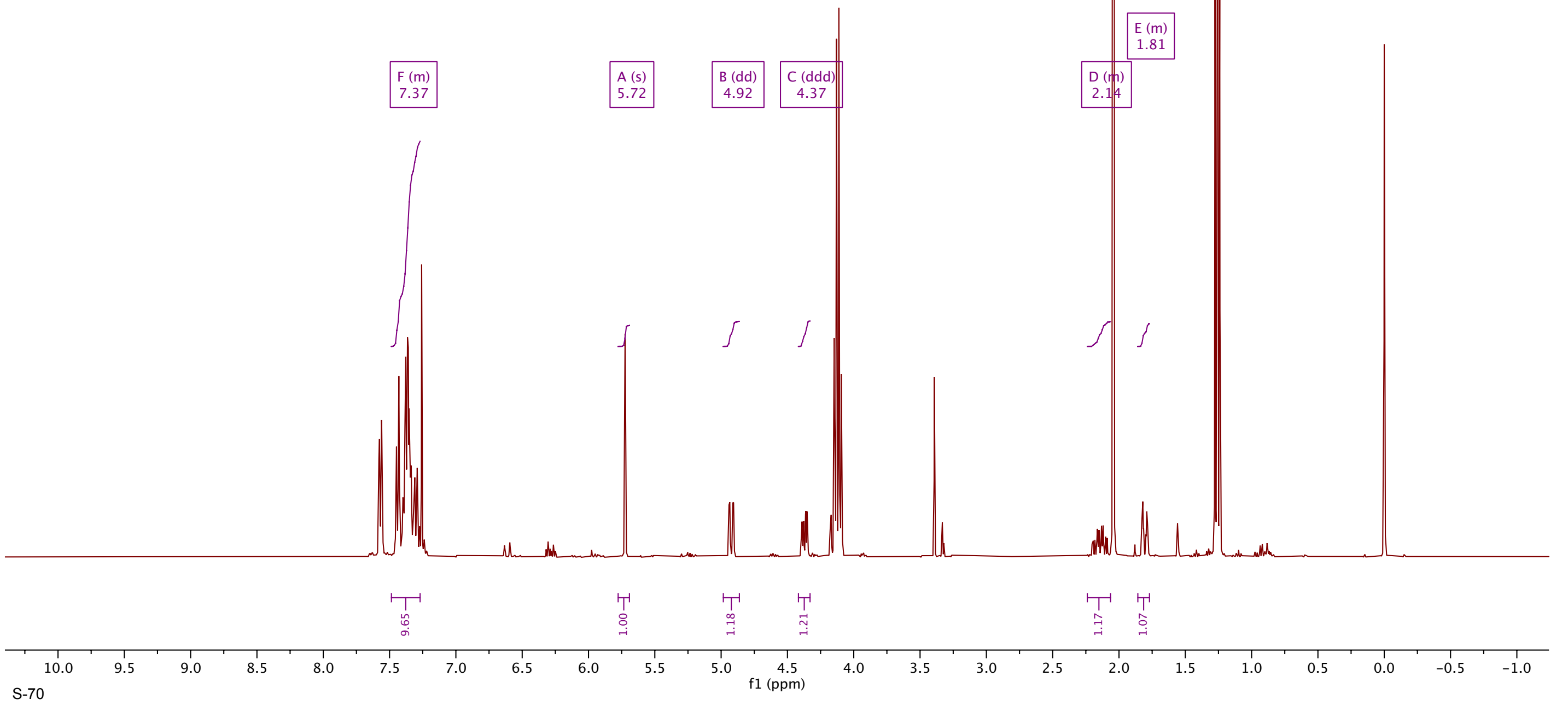




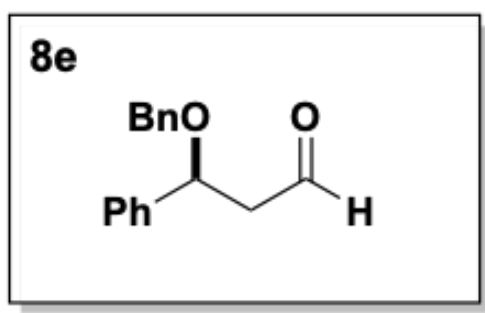

${ }^{1} \mathrm{H}$ NMR (400 MHz, $\mathrm{CDCl}_{3}$ )

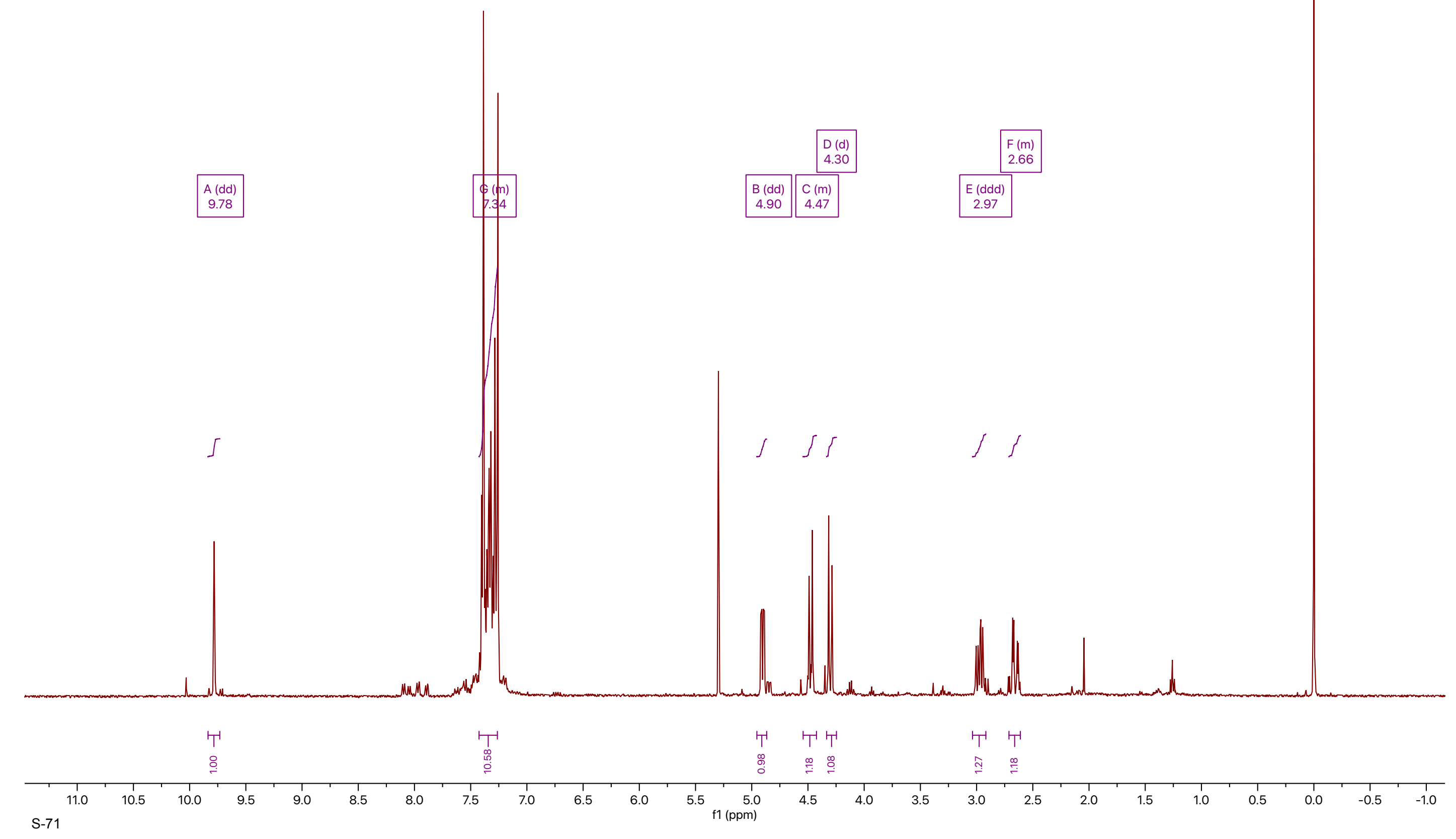




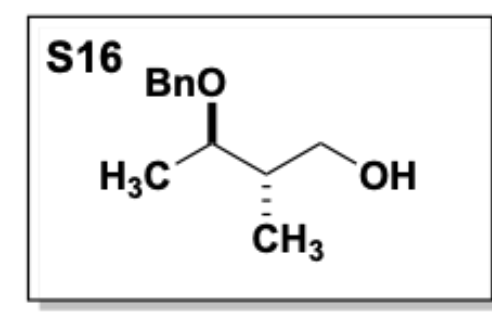

${ }^{1} \mathrm{H}$ NMR (400 MHz, $\mathrm{CDCl}_{3}$ )

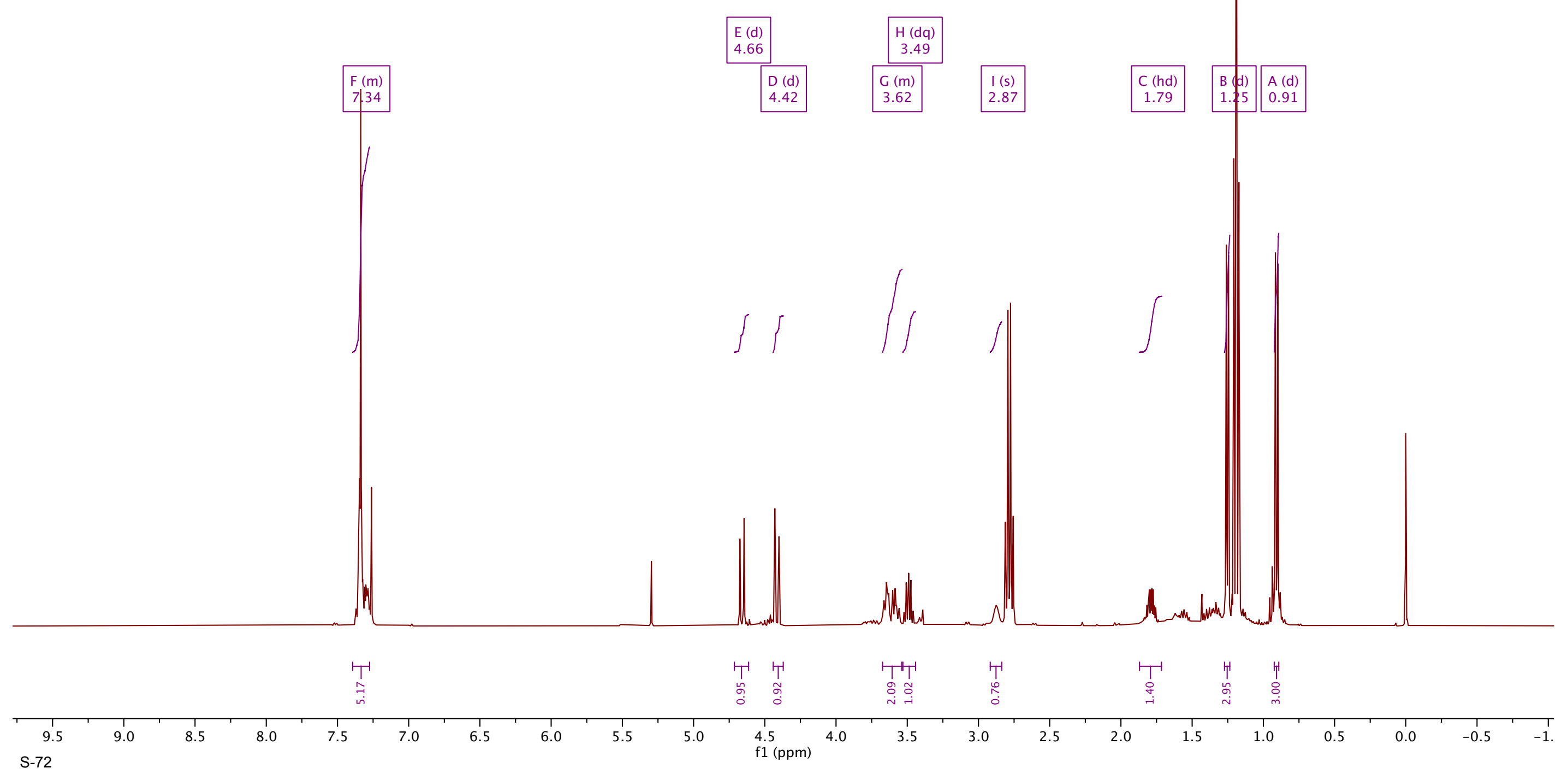




\section{Bno 0 $\overbrace{\mathrm{CH}_{3}}^{H}$}

${ }^{1} \mathrm{H}$ NMR (400 MHz, $\left.\mathrm{CDCl}_{3}\right)$

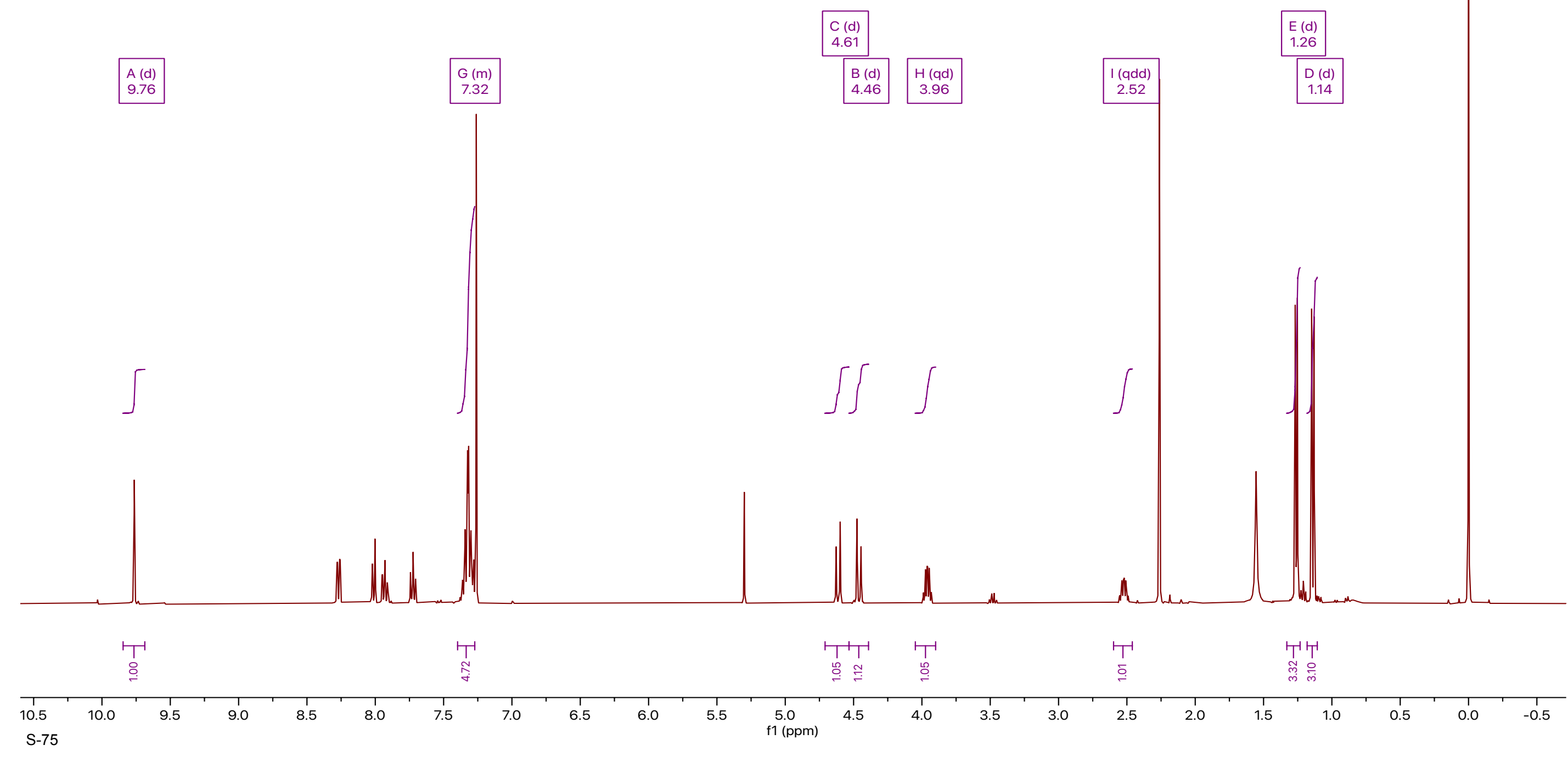




\section{Computational Methods}

All calculations were performed with Gaussian $16 .{ }^{1}$ Gas-phase ground state geometries were optimized in vacuo using Truhlar's meta-hybrid density functional B3LYP2, 3 and the 6-31G(d,p) basis set. Vibrational frequencies were computed to determine if the optimized structures are minima on the potential energy surface corresponding to ground state geometry. We have atomically truncated the $p$-tolSO $\mathrm{S}_{2}$ of the $N$-tosyl group to $\mathrm{H}_{3} \mathrm{CSO}_{2}$ for computational fidelity. We have performed a systematic conformational analysis of the initial imine reactants and the chelated intermediates and herein we report the lowest energy conformers for each species.

\section{Cartesian Coordinates and Energies}

All of the cartesian coordinates and sum of electronic and thermal free energies (Hartrees) correspond to B3LYP/6-21G(d,p)-optimized geometries.

$\mathbf{H}^{+}$

Sum of electronic and thermal Free Energies $=-0.0100000$ Hartrees
H
0.00000
$\odot .00000$
0.00000

\section{Imine (12)}

Sum of electronic and thermal Free Energies $=-1145.838400$ Hartrees

\begin{tabular}{|c|c|c|c|c|c|}
\hline \multirow{2}{*}{$\begin{array}{l}\text { Center } \\
\text { Number }\end{array}$} & \multirow{2}{*}{$\begin{array}{l}\text { Atomic } \\
\text { Number }\end{array}$} & \multirow{2}{*}{$\begin{array}{c}\text { Atomic } \\
\text { Type }\end{array}$} & \multicolumn{3}{|c|}{ Coordinates (Angstroms) } \\
\hline & & & $\mathrm{X}$ & $\mathrm{Y}$ & Z \\
\hline & & & -------- & -------- & --------- \\
\hline 1 & 6 & $\odot$ & -1.479624 & 2.059365 & 0.862294 \\
\hline 2 & 1 & $\odot$ & -1.770219 & 2.561600 & -0.065555 \\
\hline 3 & 1 & $\odot$ & -1.083127 & 2.808989 & 1.555233 \\
\hline 4 & 1 & $\odot$ & -2.376732 & 1.624350 & 1.307927 \\
\hline 5 & 6 & $\odot$ & -0.425408 & $\odot .987434$ & $\odot .581570$ \\
\hline 6 & 1 & $\odot$ & -0.158198 & 0.490729 & 1.529535 \\
\hline 7 & 6 & $\odot$ & $\odot .844222$ & 1.599208 & -0.035617 \\
\hline 8 & 1 & $\odot$ & 1. 214631 & 2.428373 & $\odot .572747$ \\
\hline 9 & 1 & $\odot$ & $\odot .570376$ & 1.998126 & -1.022713 \\
\hline 10 & 6 & $\odot$ & 1.935275 & $\odot .592819$ & -0.233907 \\
\hline 11 & 1 & $\odot$ & 1.661495 & -0.352940 & -0.713659 \\
\hline 12 & 7 & $\odot$ & 3.130270 & ๑.840972 & ๑.150979 \\
\hline
\end{tabular}




\begin{tabular}{|c|c|c|c|c|c|}
\hline 13 & 16 & $\odot$ & 4.283456 & $-\odot .397174$ & -0.226744 \\
\hline 14 & 8 & $\odot$ & 3.621048 & -1.669985 & -0.551918 \\
\hline 15 & 8 & $\odot$ & 5.245049 & ๑. 191177 & -1.162670 \\
\hline 16 & 6 & $\odot$ & 5.067312 & -0.552701 & 1. 384502 \\
\hline 17 & 1 & $\odot$ & 5.892560 & -1.255996 & 1.261311 \\
\hline 18 & 1 & $\odot$ & 5.436485 & 0.427462 & 1.685337 \\
\hline 19 & 1 & $\odot$ & 4.339413 & -0.938323 & 2.098946 \\
\hline 20 & 8 & $\odot$ & -0.862092 & -0.005691 & -0.348468 \\
\hline 21 & 6 & $\odot$ & -1.742550 & $-1.0 \odot 6947$ & $\odot .155076$ \\
\hline 22 & 1 & $\odot$ & -1.513405 & -1.907173 & -0.428534 \\
\hline 23 & 1 & $\odot$ & -1.506771 & -1.234851 & 1. 205082 \\
\hline 24 & 6 & $\odot$ & -3.216377 & -0.675845 & 0.011284 \\
\hline 25 & 6 & $\odot$ & -3.690902 & $-\odot .047456$ & -1.147216 \\
\hline 26 & 6 & $\odot$ & -4.129450 & -1.042170 & 1.005674 \\
\hline 27 & 6 & $\odot$ & -5.051650 & 0.210292 & -1.304775 \\
\hline 28 & 1 & $\odot$ & -2.982667 & ๑. 245945 & -1.915959 \\
\hline 29 & 6 & $\odot$ & -5.494003 & -0.790737 & $\odot .846782$ \\
\hline 30 & 1 & $\odot$ & -3.772364 & -1.527306 & 1.911382 \\
\hline 31 & 6 & $\odot$ & -5.958021 & -0.162653 & -0.308943 \\
\hline 32 & 1 & $\odot$ & -5.407034 & $\odot .700975$ & -2.206454 \\
\hline 33 & 1 & $\odot$ & -6.191030 & -1.079224 & 1.628314 \\
\hline 34 & 1 & $\odot$ & -7.018163 & 0.037677 & -0.433101 \\
\hline
\end{tabular}

\section{Conformer (21a)}

Sum of electronic and thermal Free Energies $=-1146.195110$ Hartrees

\begin{tabular}{|c|c|c|c|c|c|}
\hline \multirow{2}{*}{$\begin{array}{l}\text { Center } \\
\text { Number }\end{array}$} & \multirow{2}{*}{$\begin{array}{l}\text { Atomic } \\
\text { Number }\end{array}$} & \multirow{2}{*}{$\begin{array}{l}\text { Atomic } \\
\text { Type }\end{array}$} & \multicolumn{3}{|c|}{ Coordinates (Angstroms) } \\
\hline & & & $\mathrm{X}$ & $Y$ & Z \\
\hline & & & --------- & -- - - - - - - & 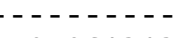 \\
\hline 1 & 6 & $\odot$ & $-\odot .086112$ & 4.123447 & $\odot .061313$ \\
\hline 2 & 1 & $\odot$ & -0.469383 & 4.398854 & 1.048272 \\
\hline 3 & 1 & $\odot$ & -0.529162 & 4.788329 & -0.686209 \\
\hline 4 & 1 & $\odot$ & $\odot .993092$ & 4.291702 & $\odot .047660$ \\
\hline 5 & 6 & $\odot$ & -0.414548 & 2.668884 & -0.255606 \\
\hline 6 & 1 & $\odot$ & $\odot .019349$ & 2.393438 & -1.229982 \\
\hline 7 & 6 & $\odot$ & -1.939885 & 2.439473 & -0.312451 \\
\hline 8 & 1 & $\odot$ & -2.389881 & 3.036312 & -1.112091 \\
\hline 9 & 1 & $\odot$ & -2.403634 & 2.794750 & 0.622667 \\
\hline 10 & 6 & $\odot$ & -2.381531 & 1.022432 & -0.476795 \\
\hline 11 & 1 & $\odot$ & -3.344361 & 0.785318 & -0.935887 \\
\hline 12 & 7 & $\odot$ & -1.667944 & ๑.031129 & -0.075036 \\
\hline 13 & 16 & $\odot$ & -2.127937 & -1.708841 & -0.442475 \\
\hline 14 & 8 & $\odot$ & -3.477833 & -1.586891 & -0.966253 \\
\hline 15 & 8 & $\odot$ & $-1 . \odot \odot 1214$ & -2.202907 & -1.210419 \\
\hline 16 & 6 & $\odot$ & -2.114913 & -2.388325 & 1.215934 \\
\hline 17 & 1 & $\odot$ & -2.368960 & -3.445404 & 1. 101071 \\
\hline 18 & 1 & $\odot$ & -1.107090 & -2.280915 & 1.622684 \\
\hline 19 & 1 & $\odot$ & -2.869168 & -1.876421 & 1.814314 \\
\hline 20 & 8 & $\odot$ & 0.077232 & 1.756144 & 0.742238 \\
\hline 21 & 6 & $\odot$ & 1. 522671 & 1.591592 & 0.823783 \\
\hline 22 & 1 & $\odot$ & 1.946309 & 2.440156 & 1.368795 \\
\hline 23 & 1 & $\odot$ & 1.937284 & 1.581521 & -0.192479 \\
\hline 24 & 1 & $\odot$ & -0.746905 & ๑. 295336 & ๑. 358504 \\
\hline 25 & 6 & $\odot$ & 1.778637 & 0.290285 & 1.534403 \\
\hline 26 & 6 & $\odot$ & 1.933014 & $\odot .255604$ & 2.925878 \\
\hline 27 & 6 & $\odot$ & 1.798635 & $-\odot .912810$ & 0.812281 \\
\hline 28 & 6 & 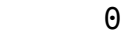 & 2.104044 & -0.960778 & 3.587220 \\
\hline 29 & 1 & $\odot$ & 1.925341 & 1.183346 & 3.491454 \\
\hline 30 & 6 & $\odot$ & 1.965115 & -2.131741 & 1.473869 \\
\hline
\end{tabular}




\begin{tabular}{|c|c|c|c|c|c|}
\hline 31 & 1 & $\odot$ & 1.710166 & -0.898853 & -0.271821 \\
\hline 32 & 6 & $\odot$ & 2.116819 & -2.155333 & 2.863203 \\
\hline 33 & 1 & $\odot$ & 2.234405 & $-\odot .976451$ & 4.664621 \\
\hline 34 & 1 & $\odot$ & 2.002814 & -3.054797 & 0.903421 \\
\hline 35 & 1 & $\odot$ & 2.262853 & -3.100165 & 3.377551 \\
\hline
\end{tabular}

\section{Conformer (22a)}

Sum of electronic and thermal Free Energies $=-1146.192942$ Hartrees

\begin{tabular}{|c|c|c|c|c|c|}
\hline \multirow{2}{*}{$\begin{array}{l}\text { Center } \\
\text { Number }\end{array}$} & \multirow{2}{*}{$\begin{array}{l}\text { Atomic } \\
\text { Number }\end{array}$} & \multirow{2}{*}{$\begin{array}{l}\text { Atomic } \\
\text { Type }\end{array}$} & \multicolumn{3}{|c|}{ Coordinates (Angstroms) } \\
\hline & & & $\mathrm{X}$ & $\mathrm{Y}$ & Z \\
\hline & - - & & --------- & - - - - - - - - & $--\cdots---$ \\
\hline 1 & 6 & $\odot$ & -2.747240 & $-\odot .717118$ & 1.479819 \\
\hline 2 & 1 & $\odot$ & -1.786725 & -0.590128 & 1.989873 \\
\hline 3 & 1 & $\theta$ & -3.145601 & -1.703597 & 1.734875 \\
\hline 4 & 1 & $\odot$ & -3.441396 & 0.026919 & 1.876709 \\
\hline 5 & 6 & $\odot$ & -2.602002 & -0.573309 & -0.035951 \\
\hline 6 & 1 & $\odot$ & -3.577454 & -0.737180 & -0.512906 \\
\hline 7 & 6 & $\odot$ & -1.619113 & -1.602346 & -0.636952 \\
\hline 8 & 1 & $\odot$ & -1.740109 & -1.647028 & -1.732630 \\
\hline 9 & 1 & $\odot$ & -1.837669 & -2.612696 & -0.276622 \\
\hline 10 & 6 & 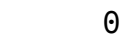 & -0.164799 & -1.333349 & -0.443491 \\
\hline 11 & 1 & $\odot$ & 0.558049 & -2.150546 & -0.508004 \\
\hline 12 & 7 & $\odot$ & 0.316971 & -0.153680 & -0.261187 \\
\hline 13 & 16 & $\odot$ & 2.132880 & $\odot .153178$ & -0.312116 \\
\hline 14 & 8 & $\odot$ & 2.685789 & -1.189352 & -0.369439 \\
\hline 15 & 8 & $\odot$ & 2.281007 & 1.143859 & -1.358534 \\
\hline 16 & 6 & $\odot$ & 2.376680 & 0.890021 & 1.304623 \\
\hline 17 & 1 & $\odot$ & 3.439284 & 1. 142058 & 1. 354388 \\
\hline 18 & 1 & $\Theta$ & 1.771401 & 1.795406 & 1.370282 \\
\hline 19 & 1 & $\odot$ & 2.120728 & 0.157396 & 2.070462 \\
\hline 20 & 8 & $\odot$ & -2.103075 & 0.715950 & -0.420780 \\
\hline 21 & 6 & $\odot$ & -3.051781 & 1.819507 & -0.420349 \\
\hline 22 & 1 & $\odot$ & -3.523057 & 1.896847 & ๑.565130 \\
\hline 23 & 1 & $\odot$ & -3.826760 & 1.594652 & -1.163346 \\
\hline 24 & 1 & $\odot$ & -0.382989 & 0.626969 & -0.266653 \\
\hline 25 & 6 & $\odot$ & -2.300179 & 3.075785 & -0.757009 \\
\hline 26 & 6 & $\odot$ & -1.889369 & 3.949733 & 0.258013 \\
\hline 27 & 6 & $\odot$ & -1.972031 & 3.368655 & -2.087967 \\
\hline 28 & 6 & $\odot$ & -1.165465 & 5.101628 & -0.052093 \\
\hline 29 & 1 & $\odot$ & -2.154452 & 3.738560 & 1. 291301 \\
\hline 30 & 6 & $\odot$ & -1.245287 & 4.516916 & -2.398218 \\
\hline 31 & 1 & $\odot$ & -2.296083 & 2.701362 & -2.882395 \\
\hline 32 & 6 & $\odot$ & -0.841561 & 5.384282 & -1.380223 \\
\hline 33 & 1 & $\Theta$ & -0.865195 & 5.782143 & 0.738650 \\
\hline 34 & 1 & $\odot$ & -1.002306 & 4.740844 & -3.432062 \\
\hline 35 & 1 & $\odot$ & -0.284634 & 6.283701 & -1.623557 \\
\hline
\end{tabular}

\section{Conformer (21b)}

Sum of electronic and thermal Free Energies $=-1264.065741$ Hartrees 


\begin{tabular}{|c|c|c|c|c|c|}
\hline \multirow{2}{*}{$\begin{array}{l}\text { Center } \\
\text { Number }\end{array}$} & \multirow{2}{*}{$\begin{array}{l}\text { Atomic } \\
\text { Number }\end{array}$} & \multirow{2}{*}{$\begin{array}{l}\text { Atomic } \\
\text { Type }\end{array}$} & \multicolumn{3}{|c|}{ Coordinates (Angstroms) } \\
\hline & & & $\mathrm{X}$ & $\mathrm{Y}$ & Z \\
\hline & & & & & ------- \\
\hline 1 & 6 & $\odot$ & -1.502565 & -0.871673 & -0.205988 \\
\hline 2 & 1 & $\odot$ & -1.364742 & -1.078591 & -1.279385 \\
\hline 3 & 6 & $\odot$ & -0.984817 & -2.089010 & $\odot .591193$ \\
\hline 4 & 1 & $\odot$ & -1.541903 & -2.991317 & $\odot .320298$ \\
\hline 5 & 1 & $\odot$ & -1.166135 & -1.936605 & 1.667180 \\
\hline 6 & 6 & $\odot$ & 0.472835 & -2.375158 & 0.460681 \\
\hline 7 & 1 & $\odot$ & 0.859708 & -3.389639 & $\odot .583098$ \\
\hline 8 & 7 & $\odot$ & 1.333332 & -1.449416 & 0.219575 \\
\hline 9 & 16 & $\odot$ & 3.095245 & -1.827709 & -0.119983 \\
\hline 10 & 8 & $\odot$ & 3.204784 & -3.232469 & 0.235734 \\
\hline 11 & 8 & $\odot$ & 3.303944 & -1.312643 & -1.459846 \\
\hline 12 & 6 & $\odot$ & 3.878613 & -0.769818 & 1.096362 \\
\hline 13 & 1 & $\odot$ & 4.953647 & -0.917536 & 0.964026 \\
\hline 14 & 1 & $\odot$ & 3.606603 & 0.264853 & $\odot .877072$ \\
\hline 15 & 1 & $\odot$ & 3. 568781 & -1.086008 & 2.092860 \\
\hline 16 & 8 & $\odot$ & -0.640253 & ๑. 223389 & ๑. 160057 \\
\hline 17 & 6 & $\odot$ & -0.742315 & 1.430526 & -0.648226 \\
\hline 18 & 1 & $\odot$ & -1.624208 & 1.995595 & -0.332318 \\
\hline 19 & 1 & $\odot$ & -0.864830 & 1.144786 & -1.700838 \\
\hline 20 & 1 & $\odot$ & ๑. 924891 & -0.488231 & 0.095235 \\
\hline 21 & 6 & $\odot$ & 0.525233 & 2.213191 & -0.435813 \\
\hline 22 & 6 & $\odot$ & ๑.609790 & 3.171954 & ๑. 581545 \\
\hline 23 & 6 & $\odot$ & 1.659081 & 1.940643 & -1.216301 \\
\hline 24 & 6 & $\odot$ & 1.807136 & 3.847727 & $\odot .817570$ \\
\hline 25 & 1 & $\odot$ & -0.265649 & 3.393899 & 1.185730 \\
\hline 26 & 6 & $\odot$ & 2.859876 & 2.613454 & -0.978057 \\
\hline 27 & 1 & $\odot$ & 1.600464 & 1.219872 & -2.028823 \\
\hline 28 & 6 & $\odot$ & 2.933890 & 3.567212 & 0.040957 \\
\hline 29 & 1 & $\odot$ & 1.859506 & 4.597077 & 1.601021 \\
\hline 30 & 1 & $\odot$ & 3.724617 & 2.411813 & -1.602913 \\
\hline 31 & 1 & $\odot$ & 3.861573 & 4.102888 & ๑. 217184 \\
\hline 32 & 6 & $\odot$ & -2.987274 & -0.588915 & ๑. 055545 \\
\hline 33 & 1 & $\odot$ & -3.299520 & 0.209954 & -0.627717 \\
\hline 34 & 1 & 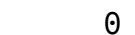 & -3.556546 & -1.474813 & -0.255458 \\
\hline 35 & 6 & $\odot$ & -3.346777 & -0.201839 & 1.496317 \\
\hline 36 & 1 & $\odot$ & -2.714620 & 0.634936 & 1.819127 \\
\hline 37 & 1 & $\odot$ & -3.127657 & -1.033284 & 2.181271 \\
\hline 38 & 6 & $\odot$ & -4.826159 & ๑. 179879 & 1.650894 \\
\hline 39 & 1 & $\odot$ & -5.053029 & 1.017374 & $\odot .978113$ \\
\hline 40 & 1 & $\odot$ & -5.452545 & -0.657197 & 1.316144 \\
\hline 41 & 6 & $\odot$ & -5.198059 & $\odot .558511$ & 3. 086818 \\
\hline 42 & 1 & $\odot$ & -4.611096 & 1.414205 & 3.438162 \\
\hline 43 & 1 & $\odot$ & -6.254924 & 0.829173 & 3.160338 \\
\hline 44 & 1 & 0 & -5.020600 & -0.273346 & 3.777249 \\
\hline
\end{tabular}

\section{Conformer (22b)}

Sum of electronic and thermal Free Energies $=-1264.064138$ Hartrees

\begin{tabular}{|c|c|c|c|c|c|}
\hline Center & Atomic & Atomic & \multicolumn{3}{|c|}{$X^{\text {Coordinates (Angstroms) }}$} \\
\hline Number & Number & Type & X & Y & Z \\
\hline 1 & 6 & $\theta$ & -0.713367 & 3.250709 & \\
\hline
\end{tabular}




\begin{tabular}{|c|c|c|c|c|c|}
\hline 2 & 1 & $\odot$ & -1.266645 & 2.477200 & 1.746944 \\
\hline 3 & 1 & $\odot$ & ๑. 255215 & 3.345804 & 1.699957 \\
\hline 4 & 6 & $\odot$ & $-\odot .447125$ & 2.767193 & $-\odot .237559$ \\
\hline 5 & 1 & $\odot$ & $\odot .014076$ & 3.580725 & -0.813962 \\
\hline 6 & 6 & $\odot$ & -1.726975 & 2.340050 & $-\odot .989704$ \\
\hline 7 & 1 & $\odot$ & -1.521217 & 2.254796 & -2.070227 \\
\hline 8 & 1 & $\odot$ & -2.511223 & 3.097570 & -0.901777 \\
\hline 9 & 6 & $\odot$ & -2.305698 & 1.015031 & -0.624896 \\
\hline 10 & 1 & $\odot$ & -3.359054 & $\odot .801937$ & -0.823501 \\
\hline 11 & 7 & $\odot$ & -1.609169 & $\odot .051774$ & -0.130325 \\
\hline 12 & 16 & $\odot$ & -2.309171 & -1.639939 & $\odot .044818$ \\
\hline 13 & 8 & $\odot$ & -3.711486 & -1.447374 & -0.284421 \\
\hline 14 & 8 & $\odot$ & -1.397479 & -2.468135 & -0.717975 \\
\hline 15 & 6 & $\odot$ & -2.083432 & -1.896498 & 1.805337 \\
\hline 16 & 1 & $\odot$ & -2.446735 & -2.908373 & 2.003136 \\
\hline 17 & 1 & $\odot$ & -1.019048 & -1.826766 & 2.034632 \\
\hline 18 & 1 & $\odot$ & -2.678324 & -1.162598 & 2.349597 \\
\hline 19 & 8 & $\odot$ & 0.420686 & 1.621401 & -0.268048 \\
\hline 20 & 6 & $\odot$ & 1.850578 & 1.869759 & -0.175810 \\
\hline 21 & 1 & $\odot$ & 2.061955 & 2.462046 & 0.720852 \\
\hline 22 & 1 & $\odot$ & 2.147988 & 2.450872 & -1.057417 \\
\hline 23 & 1 & $\odot$ & -0.586634 & $\odot .250704$ & -0.006576 \\
\hline 24 & 6 & $\odot$ & 2.549652 & $\odot .541138$ & -0.120762 \\
\hline 25 & 6 & $\odot$ & 2.952056 & ๑. .०1199 & 1. 107713 \\
\hline 26 & 6 & $\odot$ & 2.774668 & $-\odot .188442$ & -1.296303 \\
\hline 27 & 6 & $\odot$ & 3.574357 & -1.246499 & 1. 160812 \\
\hline 28 & 1 & $\odot$ & 2.794682 & $\odot .567288$ & 2.022677 \\
\hline 29 & 6 & $\odot$ & 3.392568 & -1.436899 & -1.243934 \\
\hline 30 & 1 & $\odot$ & 2.475301 & 0.228035 & -2.254656 \\
\hline 31 & 6 & $\odot$ & 3.793041 & -1.966674 & -0.014963 \\
\hline 32 & 1 & $\odot$ & 3.897401 & -1.650531 & 2.115246 \\
\hline 33 & 1 & $\odot$ & 3.570617 & -1.991626 & -2.159697 \\
\hline 34 & 1 & $\odot$ & 4.283557 & -2.934256 & $\odot .024323$ \\
\hline 35 & 6 & $\odot$ & -1.450145 & 4.595877 & 1. 288772 \\
\hline 36 & 1 & $\odot$ & -2.447871 & 4.521300 & $\odot .833974$ \\
\hline 37 & 1 & $\odot$ & $-\odot .904385$ & 5.349402 & $\odot .704711$ \\
\hline 38 & 6 & $\odot$ & -1.605014 & 5.090758 & 2.734019 \\
\hline 39 & 1 & $\odot$ & -0.610631 & 5.182141 & 3.189933 \\
\hline 40 & 1 & $\odot$ & -2.141397 & 4.332641 & 3.320409 \\
\hline 41 & 6 & $\odot$ & -2.340084 & 6.430276 & 2.830320 \\
\hline 42 & 1 & $\odot$ & -3.351550 & 6.361404 & 2.415186 \\
\hline 43 & 1 & $\odot$ & -2.430606 & 6.754344 & 3.870700 \\
\hline 44 & 1 & $\odot$ & -1.808197 & 7. 215857 & 2.283194 \\
\hline
\end{tabular}

\section{Conformer (21c)}

Sum of electronic and thermal Free Energies $=-1224.770847$ Hartrees

\begin{tabular}{|c|c|c|c|c|c|}
\hline \multirow{2}{*}{$\begin{array}{l}\text { Center } \\
\text { Number }\end{array}$} & \multirow{2}{*}{$\begin{array}{l}\text { Atomic } \\
\text { Number }\end{array}$} & \multirow{2}{*}{$\begin{array}{c}\text { Atomic } \\
\text { Type }\end{array}$} & \multicolumn{3}{|c|}{ Coordinates (Angstroms) } \\
\hline & & & $x$ & $Y$ & Z \\
\hline 1 & 6 & $\theta$ & -2.204013 & -0.405502 & $-\odot .34607$ \\
\hline 2 & 1 & 0 & -2.125455 & -0.525617 & -1.43800 \\
\hline 3 & 6 & 0 & -1.842728 & -1.751667 & 0.332921 \\
\hline 4 & 1 & 0 & -2.483521 & -2.556524 & -0.033584 \\
\hline 5 & 1 & 0 & -2.052525 & -1.676246 & 1.41357 \\
\hline 6 & 6 & $\theta$ & -0.430832 & -2.216910 & 0.239290 \\
\hline 7 & 1 & 0 & -0.199531 & -3.284676 & 0.264449 \\
\hline 8 & 7 & $\theta$ & 0.572863 & -1.416904 & 0.160682 \\
\hline
\end{tabular}




\begin{tabular}{|c|c|c|c|c|c|}
\hline 9 & 16 & $\odot$ & 2. 280977 & $-2.052 \odot 99$ & $-\odot .105711$ \\
\hline 10 & 8 & $\odot$ & 2.139949 & -3.472633 & ๑. 167368 \\
\hline 11 & 8 & $\odot$ & 2.661653 & -1.514458 & -1.397414 \\
\hline 12 & 6 & $\odot$ & 3.132992 & -1.201207 & 1. 221187 \\
\hline 13 & 1 & $\odot$ & 4.176034 & -1.520664 & 1.149533 \\
\hline 14 & 1 & $\odot$ & 3.052360 & -0.125370 & 1.050806 \\
\hline 15 & 1 & $\odot$ & 2.702430 & -1.513705 & 2.173023 \\
\hline 16 & 8 & $\odot$ & -1.201554 & 0.535630 & ๑.087223 \\
\hline 17 & 6 & $\odot$ & -0.819120 & 1.601504 & -0.833292 \\
\hline 18 & 1 & $\odot$ & -1.535374 & 2.421215 & -0.749915 \\
\hline 19 & 1 & $\odot$ & -0.846257 & 1.222314 & -1.862274 \\
\hline 20 & 1 & $\odot$ & 0.337018 & -0.400950 & 0.125549 \\
\hline 21 & 6 & $\odot$ & $\odot .570464$ & 2.045514 & -0.455023 \\
\hline 22 & 6 & $\Theta$ & $\odot .783945$ & 2.724163 & 0.755729 \\
\hline 23 & 6 & $\odot$ & 1.665738 & 1.760558 & -1.281479 \\
\hline 24 & 6 & $\odot$ & 2.069398 & 3.109439 & 1. 131094 \\
\hline 25 & 1 & $\odot$ & -0.061035 & 2.954962 & 1.398404 \\
\hline 26 & 6 & $\odot$ & 2. 955019 & 2. 152530 & -0.908452 \\
\hline 27 & 1 & $\odot$ & 1. 514331 & 1.244346 & -2.225640 \\
\hline 28 & 6 & $\odot$ & 3.157457 & 2.825513 & $\odot .298002$ \\
\hline 29 & 1 & $\odot$ & 2.223357 & 3.644013 & 2.063248 \\
\hline 30 & 1 & $\odot$ & 3.792852 & 1.941630 & -1.565479 \\
\hline 31 & 1 & $\odot$ & 4.155911 & 3.143479 & ๑. 582399 \\
\hline 32 & 6 & $\odot$ & -3.653149 & $-\odot .0 \odot 2846$ & 0.021005 \\
\hline 33 & 6 & $\odot$ & -4.666057 & $-1.0 \odot 3652$ & -0.567239 \\
\hline 34 & 1 & $\odot$ & -5.683082 & -0.685468 & -0.324874 \\
\hline 35 & 1 & $\odot$ & -4.589587 & -1.048370 & -1.659826 \\
\hline 36 & 1 & $\odot$ & -4.552394 & -2.019073 & -0.176440 \\
\hline 37 & 6 & $\odot$ & $-4.0 \odot 9381$ & 1.423392 & -0.423298 \\
\hline 38 & 1 & $\odot$ & -3.420164 & 2.174849 & 0.106042 \\
\hline 39 & 1 & $\odot$ & -3.872488 & 1.560887 & -1.501942 \\
\hline $4 \odot$ & 1 & $\odot$ & -5.061148 & 1.623305 & -0.201457 \\
\hline 41 & 1 & $\odot$ & -3.723015 & -0.036161 & 1. 117552 \\
\hline
\end{tabular}

\section{Conformer (22c)}

Sum of electronic and thermal Free Energies $=-1224.772123$ Hartrees

\begin{tabular}{|c|c|c|c|c|c|}
\hline \multirow{2}{*}{$\begin{array}{l}\text { Center } \\
\text { Number }\end{array}$} & \multirow{2}{*}{$\begin{array}{l}\text { Atomic } \\
\text { Number }\end{array}$} & \multirow{2}{*}{$\begin{array}{c}\text { Atomic } \\
\text { Type }\end{array}$} & \multicolumn{3}{|c|}{ Coordinates (Angstroms) } \\
\hline & & & $\mathrm{X}$ & $\mathrm{Y}$ & Z \\
\hline 1 & 6 & $\odot$ & 1.690540 & -0.487441 & -1.301660 \\
\hline 2 & 1 & $\Theta$ & 2.067760 & -1.223802 & -2.023204 \\
\hline 3 & 6 & $\odot$ & 1.339356 & 0.801684 & -2.092859 \\
\hline 4 & 1 & $\odot$ & 0.740842 & 0.543414 & -2.983158 \\
\hline 5 & 1 & $\odot$ & 2.230229 & 1.296940 & -2.486329 \\
\hline 6 & 6 & $\odot$ & ๑.506941 & 1.802711 & -1.367531 \\
\hline 7 & 1 & $\odot$ & $\odot .535501$ & 2.859970 & -1.642778 \\
\hline 8 & 7 & $\odot$ & -0.320914 & 1.469467 & -0.438474 \\
\hline 9 & 16 & $\odot$ & -1.529617 & 2.673832 & ๑. 241948 \\
\hline 10 & 8 & $\odot$ & -1.113426 & 3.929452 & -0.359961 \\
\hline 11 & 8 & $\odot$ & -2.810659 & 2.040068 & $-0.00 \odot 230$ \\
\hline 12 & 6 & $\odot$ & -1.103638 & 2.611127 & 1.982454 \\
\hline 13 & 1 & $\odot$ & -1.806496 & 3.283892 & 2.480892 \\
\hline 14 & 1 & $\theta$ & -1.241613 & 1.589469 & 2.339538 \\
\hline 15 & 1 & $\odot$ & -0.079872 & 2.964328 & 2.108831 \\
\hline 16 & 8 & $\odot$ & 0.490663 & -0.994683 & -0.688067 \\
\hline 17 & 6 & $\odot$ & $-\odot .271485$ & -1.968869 & -1.462815 \\
\hline 18 & 1 & $\odot$ & $\odot .359104$ & -2.853705 & -1.600907 \\
\hline
\end{tabular}




\begin{tabular}{|c|c|c|c|c|c|}
\hline 19 & 1 & $\odot$ & $-\odot .5 \odot 4943$ & -1.549783 & -2.450686 \\
\hline 20 & 1 & $\odot$ & -0.359515 & 0.440609 & -0.223234 \\
\hline 21 & 6 & $\odot$ & -1.525287 & -2.288375 & -0.701399 \\
\hline 22 & 6 & $\odot$ & -1.528370 & -3.306733 & 0.260932 \\
\hline 23 & 6 & $\odot$ & -2.692271 & -1.540971 & $-\odot .911972$ \\
\hline 24 & 6 & $\odot$ & -2.680226 & -3.574185 & 0.999239 \\
\hline 25 & 1 & $\odot$ & -0.630110 & -3.896136 & 0.424570 \\
\hline 26 & 6 & $\odot$ & -3.844312 & -1.804969 & -0.170623 \\
\hline 27 & 1 & $\odot$ & -2.709635 & -0.761258 & -1.670119 \\
\hline 28 & 6 & $\odot$ & -3.838129 & -2.822562 & 0.785015 \\
\hline 29 & 1 & $\odot$ & -2.677637 & -4.371855 & 1.735319 \\
\hline 30 & 1 & $\odot$ & -4.745349 & -1.226323 & -0.347089 \\
\hline 31 & 1 & $\odot$ & -4.736683 & -3.036475 & 1. 355282 \\
\hline 32 & 6 & $\Theta$ & 2.756564 & $-\odot .291088$ & -0.202679 \\
\hline 33 & 1 & $\odot$ & 2.356158 & 0.430247 & 0.525963 \\
\hline 34 & 6 & $\odot$ & 3.007748 & -1.617906 & 0.529960 \\
\hline 35 & 1 & $\odot$ & 3.722457 & -1.470200 & 1.343956 \\
\hline 36 & 1 & $\odot$ & 2.086624 & -2.024134 & $\odot .951911$ \\
\hline 37 & 1 & $\odot$ & 3.434275 & -2.364058 & -0.150483 \\
\hline 38 & 6 & $\odot$ & 4.070966 & $\odot .270336$ & -0.770509 \\
\hline 39 & 1 & $\Theta$ & 4.466548 & -0.372560 & -1.565016 \\
\hline 40 & 1 & $\odot$ & 3.972783 & 1.284232 & -1.170856 \\
\hline 41 & 1 & $\odot$ & 4.826434 & ๑. 313223 & ๑.018155 \\
\hline
\end{tabular}

\section{Conformer (21d)}

Sum of electronic and thermal Free Energies $=-1264.059615$ Hartrees

\begin{tabular}{|c|c|c|c|c|c|}
\hline \multirow{2}{*}{$\begin{array}{l}\text { Center } \\
\text { Number }\end{array}$} & \multirow{2}{*}{$\begin{array}{l}\text { Atomic } \\
\text { Number }\end{array}$} & \multirow{2}{*}{$\begin{array}{c}\text { Atomic } \\
\text { Type }\end{array}$} & \multicolumn{3}{|c|}{ Coordinates (Angstroms) } \\
\hline & & & $\mathrm{x}$ & Y & Z \\
\hline 1 & 6 & $\odot$ & -1.660198 & -0.718083 & -0.32089 \\
\hline 2 & 1 & $\odot$ & -1.455318 & -0.910610 & -1.386093 \\
\hline 3 & 6 & $\Theta$ & -1.125858 & -1.920049 & 0.496760 \\
\hline 4 & 1 & $\theta$ & -1.596234 & -2.851550 & 0.174685 \\
\hline 5 & 1 & $\odot$ & -1.402023 & -1.802560 & 1.557566 \\
\hline 6 & 6 & $\odot$ & 0.345532 & -2.145322 & 0.498190 \\
\hline 7 & 1 & $\odot$ & 0.743717 & -3.149679 & 0.662380 \\
\hline 8 & 7 & $\odot$ & 1.212443 & -1.208974 & 0.338147 \\
\hline 9 & 16 & 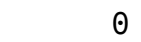 & 3.007325 & -1.582467 & $\odot .189982$ \\
\hline 10 & 8 & $\odot$ & 3.080474 & -2.975807 & 0.597682 \\
\hline 11 & 8 & $\odot$ & 3.357843 & -1.110983 & -1.135499 \\
\hline 12 & 6 & $\odot$ & 3.653336 & -0.488540 & 1.453650 \\
\hline 13 & 1 & 0 & 4.737157 & -0.630017 & 1.435200 \\
\hline 14 & 1 & $\odot$ & 3.399342 & 0.539930 & 1.187566 \\
\hline 15 & 1 & $\odot$ & 3.245293 & -0.788545 & 2.419301 \\
\hline 16 & 8 & $\odot$ & -0.857744 & $\odot .401083$ & 0.107668 \\
\hline 17 & 6 & $\odot$ & -0.708501 & 1.548381 & -0.777934 \\
\hline 18 & 1 & $\odot$ & -1.496571 & 2.271009 & -0.553160 \\
\hline 19 & 1 & $\odot$ & -0.816633 & 1.226116 & -1.819631 \\
\hline 20 & 1 & $\odot$ & 0.823294 & -0.254569 & 0.179007 \\
\hline 21 & 6 & $\odot$ & 0.653908 & 2.144150 & -0.529280 \\
\hline 22 & 6 & $\Theta$ & 0.899419 & 2.861652 & 0.652502 \\
\hline 23 & 6 & $\odot$ & 1.697734 & 1.961423 & -1.446914 \\
\hline 24 & 6 & $\theta$ & 2.164688 & 3.388335 & 0.908769 \\
\hline 25 & 1 & $\odot$ & 0.092993 & 3.017164 & 1.364007 \\
\hline 26 & 6 & $\Theta$ & 2.965213 & 2.492433 & -1.193297 \\
\hline 27 & 1 & $\odot$ & 1.518976 & 1.417168 & -2.370500 \\
\hline
\end{tabular}




$\begin{array}{rrrrrr}28 & 6 & 0 & 3.199358 & 3.205907 & -0.015916 \\ 29 & 1 & 0 & 2.340565 & 3.956221 & 1.817171 \\ 30 & 1 & 0 & 3.761192 & 2.356390 & -1.918269 \\ 31 & 1 & 0 & 4.178827 & 3.634260 & 0.174155 \\ 32 & 6 & 0 & -3.202302 & -0.525547 & -0.147154 \\ 33 & 6 & 0 & -3.538254 & -0.052113 & 1.278892 \\ 34 & 1 & 0 & -3.012490 & 0.873748 & 1.526695 \\ 35 & 1 & 0 & -4.612302 & 0.135380 & 1.364986 \\ 36 & 1 & 0 & -3.286778 & -0.799551 & 2.040009 \\ 37 & 6 & 0 & -3.919108 & -1.863406 & -0.445034 \\ 38 & 1 & 0 & -4.999189 & -1.698294 & -0.478707 \\ 39 & 1 & 0 & -3.624948 & -2.277165 & -1.416854 \\ 40 & 1 & 0 & -3.741631 & -2.622077 & 0.323178 \\ 41 & 6 & 0 & -3.722974 & 0.511333 & -1.167609 \\ 42 & 1 & 0 & -3.383564 & 1.525414 & -0.949492 \\ 43 & 1 & 0 & -3.426491 & 0.258713 & -2.191666 \\ 44 & 1 & 0 & -4.816032 & 0.531062 & -1.140728 \\ --------------\ldots\end{array}$

\section{Conformer (22d)}

Sum of electronic and thermal Free Energies $=-1264.060475$ Hartrees

\begin{tabular}{|c|c|c|c|c|c|}
\hline \multirow{2}{*}{$\begin{array}{l}\text { Center } \\
\text { Number }\end{array}$} & \multirow{2}{*}{$\begin{array}{l}\text { Atomic } \\
\text { Number }\end{array}$} & \multirow{2}{*}{$\begin{array}{c}\text { Atomic } \\
\text { Type }\end{array}$} & \multicolumn{3}{|c|}{ Coordinates (Angstroms) } \\
\hline & & & $\mathrm{X}$ & $Y$ & Z \\
\hline & & & & & \\
\hline 1 & 6 & $\odot$ & 1.682440 & -0.451969 & -1.240089 \\
\hline 2 & 1 & $\odot$ & 1.989996 & -1.138521 & -2.037697 \\
\hline 3 & 6 & 0 & 1.376496 & 0.913544 & -1.920610 \\
\hline 4 & 1 & $\theta$ & 0.866011 & 0.734832 & -2.882758 \\
\hline 5 & 1 & $\odot$ & 2.291944 & 1.447869 & -2.184892 \\
\hline 6 & 6 & $\odot$ & 0.462442 & 1.851889 & -1.211987 \\
\hline 7 & 1 & $\odot$ & $\odot .517292$ & 2.925907 & -1.406361 \\
\hline 8 & 7 & $\odot$ & -0.473804 & 1.458026 & -0.419802 \\
\hline 9 & 16 & $\odot$ & -1.751951 & 2.614390 & ๑. 199364 \\
\hline 10 & 8 & $\odot$ & -1.270916 & 3.908938 & -0.254117 \\
\hline 11 & 8 & $\odot$ & -2.997437 & 2.013007 & -0.235653 \\
\hline 12 & 6 & $\odot$ & -1.533042 & 2.419542 & 1.968138 \\
\hline 13 & 1 & $\odot$ & -2.280669 & 3.069388 & 2.430353 \\
\hline 14 & 1 & $\theta$ & -1.724251 & 1.377852 & 2.230159 \\
\hline 15 & 1 & 0 & -0.526390 & 2.740466 & 2.237369 \\
\hline 16 & 8 & $\odot$ & 0.469553 & -0.949137 & -0.641760 \\
\hline 17 & 6 & $\odot$ & $-\odot .216786$ & -2.019055 & -1.359511 \\
\hline 18 & 1 & $\odot$ & 0.437220 & -2.897099 & -1.366564 \\
\hline 19 & 1 & $\odot$ & $-\odot .391508$ & -1.703517 & -2.396360 \\
\hline 20 & 1 & $\odot$ & -0.514219 & 0.421284 & -0.260871 \\
\hline 21 & 6 & $\odot$ & -1.510830 & -2.292895 & -0.648435 \\
\hline 22 & 6 & $\theta$ & -1.569003 & -3.243584 & 0.379019 \\
\hline 23 & 6 & $\odot$ & -2.663550 & -1.562856 & -0.971961 \\
\hline 24 & 6 & $\odot$ & -2.760269 & -3.462394 & 1.069847 \\
\hline 25 & 1 & $\Theta$ & -0.682662 & -3.819994 & 0.630371 \\
\hline 26 & 6 & $\theta$ & -3.854437 & -1.776283 & -0.276971 \\
\hline 27 & 1 & $\odot$ & -2.638896 & -0.838769 & -1.783267 \\
\hline 28 & 6 & $\odot$ & -3.902665 & -2.727482 & $\odot .743971$ \\
\hline 29 & 1 & $\odot$ & -2.799770 & -4.209392 & 1.856340 \\
\hline 30 & 1 & $\odot$ & -4.742939 & -1.211765 & -0.540966 \\
\hline 31 & 1 & $\odot$ & -4.831415 & -2.903689 & 1. 277612 \\
\hline 32 & 6 & $\odot$ & 2.830319 & -0.427314 & -0.189491 \\
\hline
\end{tabular}




\begin{tabular}{|c|c|c|c|c|c|}
\hline 33 & 6 & $\odot$ & 2.526086 & 0.536688 & $\odot .971534$ \\
\hline 34 & 1 & $\odot$ & 3.335343 & ๑. 502738 & 1.706303 \\
\hline 35 & 1 & $\odot$ & 2.451968 & 1.580271 & 0.638346 \\
\hline 36 & 1 & $\odot$ & 1.600754 & 0.263011 & 1.488259 \\
\hline 37 & 6 & $\odot$ & 2.988592 & -1.859362 & 0.363351 \\
\hline 38 & 1 & $\Theta$ & 3.229097 & -2.569755 & -0.435310 \\
\hline 39 & 1 & $\odot$ & 3.806687 & -1.892566 & 1.088267 \\
\hline 40 & 1 & $\odot$ & 2.077940 & -2.196795 & 0.863658 \\
\hline 41 & 6 & $\odot$ & 4.148096 & -0.013525 & -0.878063 \\
\hline 42 & 1 & $\odot$ & 4.980738 & $-\odot .129519$ & -0.178770 \\
\hline 43 & 1 & $\odot$ & 4.364203 & $-\odot .641085$ & -1.749541 \\
\hline 44 & 1 & $\odot$ & 4.151577 & 1.032902 & -1.201059 \\
\hline
\end{tabular}

\section{Conformer (21e)}

Sum of electronic and thermal Free Energies $=-1337.889244$ Hartrees

\begin{tabular}{|c|c|c|c|c|c|}
\hline \multirow{2}{*}{$\begin{array}{l}\text { Center } \\
\text { Number }\end{array}$} & \multirow{2}{*}{$\begin{array}{l}\text { Atomic } \\
\text { Number }\end{array}$} & \multirow{2}{*}{$\begin{array}{c}\text { Atomic } \\
\text { Type }\end{array}$} & \multicolumn{3}{|c|}{ Coordinates (Angstroms) } \\
\hline & & & $x$ & $Y$ & Z \\
\hline 1 & 6 & 0 & 1.788101 & -0.019213 & -0.941667 \\
\hline 2 & 1 & $\odot$ & 1.324752 & -0.293462 & -1.901832 \\
\hline 3 & 6 & $\odot$ & 1.880543 & 1.524957 & -0.866181 \\
\hline 4 & 1 & $\theta$ & 2.479602 & 1.909997 & -1.696958 \\
\hline 5 & 1 & $\theta$ & 2.421295 & 1.815761 & 0.048363 \\
\hline 6 & 6 & $\odot$ & 0.576868 & 2.252520 & -0.833806 \\
\hline 7 & 1 & 0 & 0.491983 & 3.274243 & -1.211647 \\
\hline 8 & 7 & $\odot$ & -0.489353 & 1.716983 & -0.352280 \\
\hline 9 & 16 & $\theta$ & -2.128680 & 2.525103 & -0.476473 \\
\hline 10 & 8 & $\theta$ & -1.804843 & 3.862878 & -0.942553 \\
\hline 11 & 8 & 0 & -2.921291 & 1.573021 & -1.231226 \\
\hline 12 & 6 & 0 & -2.606340 & 2.518834 & 1.251113 \\
\hline 13 & 1 & $\odot$ & -3.601894 & 2.969263 & 1.281957 \\
\hline 14 & 1 & $\odot$ & -2.646718 & 1.482084 & 1.592046 \\
\hline 15 & 1 & $\odot$ & -1.895684 & 3.121482 & 1.817465 \\
\hline 16 & 8 & $\odot$ & 0.901517 & -0.399220 & 0.128400 \\
\hline 17 & 6 & $\theta$ & 0.480342 & -1.795550 & 0.143306 \\
\hline 18 & 1 & 0 & 1.316353 & -2.408097 & 0.491078 \\
\hline 19 & 1 & $\theta$ & 0.225345 & -2.097375 & -0.880444 \\
\hline 20 & 1 & $\theta$ & -0.367867 & 0.728122 & -0.009278 \\
\hline 21 & 6 & $\odot$ & -0.711425 & -1.882571 & 1.056405 \\
\hline 22 & 6 & $\odot$ & -0.551594 & -2.184734 & 2.414208 \\
\hline 23 & 6 & $\odot$ & -1.994838 & -1.597831 & 0.564918 \\
\hline 24 & 6 & $\odot$ & -1.654223 & -2.201879 & 3.268916 \\
\hline 25 & 1 & 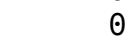 & 0.437659 & -2.413448 & 2.801097 \\
\hline 26 & 6 & $\odot$ & -3.098791 & -1.609841 & 1.420426 \\
\hline 27 & 1 & $\odot$ & -2.138443 & -1.391860 & -0.493670 \\
\hline 28 & 6 & $\odot$ & -2.927588 & -1.911112 & 2.774529 \\
\hline 29 & 1 & $\odot$ & -1.521346 & -2.448180 & 4.317620 \\
\hline 30 & 1 & $\theta$ & -4.090793 & -1.412412 & 1.025384 \\
\hline 31 & 1 & $\Theta$ & -3.786393 & -1.936203 & 3.438141 \\
\hline 32 & 6 & 0 & 3.155774 & -0.660109 & -0.849049 \\
\hline 33 & 6 & 0 & 3.749138 & -1.214225 & -1.988297 \\
\hline 34 & 6 & 0 & 3.847495 & -0.680550 & 0.370394 \\
\hline 35 & 6 & 0 & 5.025705 & -1.773926 & -1.914720 \\
\hline 36 & 1 & 0 & 3.215003 & -1.211455 & -2.935394 \\
\hline 37 & 6 & 0 & 5.118333 & -1.247382 & 0.444032 \\
\hline 38 & 1 & 0 & 3.382893 & -0.271120 & 1.263508 \\
\hline
\end{tabular}




\begin{tabular}{|c|c|c|c|c|c|}
\hline 39 & 6 & $\theta$ & 5.710372 & -1.790806 & -0.699465 \\
\hline 40 & 1 & $\odot$ & 5.479568 & -2.201278 & -2.803010 \\
\hline 41 & 1 & $\odot$ & 5.647084 & -1.266898 & 1.391743 \\
\hline 42 & 1 & 0 & 6.701089 & -2.230014 & -0.64045 \\
\hline
\end{tabular}

\section{Conformer (22e)}

Sum of electronic and thermal Free Energies $=-1337.885697$ Hartrees

\begin{tabular}{|c|c|c|c|c|c|}
\hline \multirow{2}{*}{$\begin{array}{l}\text { Center } \\
\text { Number }\end{array}$} & \multirow{2}{*}{$\begin{array}{l}\text { Atomic } \\
\text { Number }\end{array}$} & \multirow{2}{*}{$\begin{array}{l}\text { Atomic } \\
\text { Type }\end{array}$} & \multicolumn{3}{|c|}{ Coordinates (Angstroms) } \\
\hline & & & $\mathrm{x}$ & $\mathrm{Y}$ & Z \\
\hline 1 & 6 & $\odot$ & -0.388055 & 2.807642 & -0.149665 \\
\hline 2 & 1 & $\odot$ & 0.085222 & 3.644449 & -0.681314 \\
\hline 3 & 6 & 0 & -1.730960 & 2.528912 & -0.879983 \\
\hline 4 & 1 & $\theta$ & -1.568695 & 2.536046 & -1.970998 \\
\hline 5 & 1 & 0 & -2.449980 & 3.325830 & -0.672855 \\
\hline 6 & 6 & 0 & -2.379901 & 1.212995 & -0.612339 \\
\hline 7 & 1 & 0 & -3.463726 & 1.101705 & -0.695166 \\
\hline 8 & 7 & 0 & -1.711407 & 0.147014 & -0.341315 \\
\hline 9 & 16 & $\theta$ & -2.512122 & -1.504407 & -0.271849 \\
\hline 10 & 8 & 0 & -3.927125 & -1.185878 & -0.358268 \\
\hline 11 & 8 & $\theta$ & -1.788464 & -2.279095 & -1.259587 \\
\hline 12 & 6 & $\theta$ & -2.049779 & -1.999527 & 1.388760 \\
\hline 13 & 1 & 0 & -2.453812 & -3.006762 & 1.519498 \\
\hline 14 & 1 & 0 & -0.960376 & -2.017888 & 1.454849 \\
\hline 15 & 1 & 0 & -2.501381 & -1.308622 & 2.101251 \\
\hline 16 & 8 & 0 & 0.398152 & 1.626327 & -0.320880 \\
\hline 17 & 6 & $\odot$ & 1.823530 & 1.736831 & -0.036346 \\
\hline 18 & 1 & 0 & 1.957059 & 2. 284062 & 0.901605 \\
\hline 19 & 1 & 0 & 2.285101 & 2.306876 & -0.851064 \\
\hline 20 & 1 & $\theta$ & -0.672580 & 0.264338 & -0.294621 \\
\hline 21 & 6 & 0 & 2.371366 & 0.340671 & 0.057620 \\
\hline 22 & 6 & $\odot$ & 2.505341 & -0.284712 & 1.305416 \\
\hline 23 & 6 & 0 & 2.704896 & -0.370170 & -1.103223 \\
\hline 24 & 6 & $\theta$ & 2.967100 & -1.599006 & 1.390967 \\
\hline 25 & 1 & $\odot$ & 2.270897 & 0.268369 & 2.211773 \\
\hline 26 & 6 & 0 & 3.164097 & -1.684376 & -1.018712 \\
\hline 27 & 1 & $\odot$ & 2.614817 & 0.110644 & -2.073804 \\
\hline 28 & 6 & 0 & 3.294611 & -2.299980 & 0.228166 \\
\hline 29 & 1 & $\odot$ & 3.087322 & -2.069070 & 2.362299 \\
\hline 30 & 1 & $\theta$ & 3.428505 & -2.224181 & -1.922433 \\
\hline 31 & 1 & 0 & 3.662549 & -3.319151 & 0.293947 \\
\hline 32 & 6 & $\theta$ & -0.596708 & 3.207348 & 1.304622 \\
\hline 33 & 6 & 0 & -0.855296 & 4.550081 & 1.607960 \\
\hline 34 & 6 & 0 & -0.579408 & 2.265832 & 2.341212 \\
\hline 35 & 6 & 0 & -1.109755 & 4.943406 & 2.921565 \\
\hline 36 & 1 & 0 & -0.851231 & 5.295756 & 0.816080 \\
\hline 37 & 6 & $\odot$ & -0.824538 & 2.661184 & 3.657212 \\
\hline 38 & 1 & $\odot$ & -0.340641 & 1.228794 & 2.125492 \\
\hline 39 & 6 & 0 & -1.096568 & 3.998508 & 3.949027 \\
\hline 40 & 1 & 0 & -1.305755 & 5.987527 & 3.143317 \\
\hline 41 & 1 & $\odot$ & -0.794801 & 1.926842 & 4.456407 \\
\hline 42 & 1 & 0 & -1.285101 & 4.304898 & 4.972845 \\
\hline
\end{tabular}




\section{Conformer (21f)}

Sum of electronic and thermal Free Energies $=-1185.485546$ Hartrees

\begin{tabular}{|c|c|c|c|c|c|}
\hline \multirow{2}{*}{$\begin{array}{l}\text { Center } \\
\text { Number }\end{array}$} & \multirow{2}{*}{$\begin{array}{l}\text { Atomic } \\
\text { Number }\end{array}$} & \multirow{2}{*}{$\begin{array}{l}\text { Atomic } \\
\text { Type }\end{array}$} & \multicolumn{3}{|c|}{ Coordinates (Angstroms) } \\
\hline & & & x & $Y$ & z \\
\hline 1 & 6 & $\odot$ & $\odot .834859$ & -2.619966 & -0.233778 \\
\hline 2 & 6 & $\odot$ & 2.113871 & -1.902667 & -0.746867 \\
\hline 3 & 1 & 0 & 2.110613 & -1.969117 & -1.850318 \\
\hline 4 & 6 & 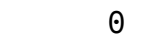 & 2.144771 & -0.420647 & -0.504581 \\
\hline 5 & 1 & 0 & 3.107392 & 0.097223 & -0.476833 \\
\hline 6 & 7 & $\odot$ & 1.097195 & 0.312469 & -0.371623 \\
\hline 7 & 16 & 0 & 1.203292 & 2.147304 & -0.336270 \\
\hline 8 & 8 & 0 & 2.634306 & 2.391013 & -0.255793 \\
\hline 9 & 8 & 0 & 0.370280 & 2.559610 & -1.448334 \\
\hline 10 & 6 & 0 & 0.392247 & 2.478356 & 1.227488 \\
\hline 11 & 1 & $\theta$ & 0.350359 & 3.567597 & 1.309560 \\
\hline 12 & 1 & 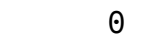 & -0.615992 & 2.058933 & 1.196039 \\
\hline 13 & 1 & 0 & 0.996526 & 2.057396 & 2.031571 \\
\hline 14 & 8 & 0 & -0.283967 & -1.828490 & -0.672800 \\
\hline 15 & 6 & 0 & -1.579605 & -2.144568 & -0.086668 \\
\hline 16 & 1 & 0 & -1.431983 & -2.612549 & 0.893572 \\
\hline 17 & 1 & 0 & -2.085855 & -2.860185 & -0.742146 \\
\hline 18 & 1 & 0 & 0.184705 & -0.199243 & -0.459186 \\
\hline 19 & 6 & $\theta$ & -2.353456 & -0.858733 & 0.045879 \\
\hline 20 & 6 & 0 & -2.587746 & -0.292808 & 1.306745 \\
\hline 21 & 6 & 0 & -2.811467 & -0.190320 & -1.099979 \\
\hline 22 & 6 & 0 & -3.275190 & 0.918675 & 1.422022 \\
\hline 23 & 1 & 0 & -2.255753 & -0.812576 & 2.202249 \\
\hline 24 & 6 & $\odot$ & -3.485712 & 1.024949 & -0.986162 \\
\hline 25 & 1 & 0 & -2.644816 & -0.626797 & -2.081025 \\
\hline 26 & 6 & $\theta$ & -3.719941 & 1.580287 & 0.274742 \\
\hline 27 & 1 & $\odot$ & -3.477786 & 1.334229 & 2.404609 \\
\hline 28 & 1 & 0 & -3.839137 & 1.532196 & -1.878051 \\
\hline 29 & 1 & 0 & -4.260562 & 2.517487 & 0.362850 \\
\hline 30 & 6 & 0 & 3.417872 & -2.543565 & -0.229809 \\
\hline 31 & 1 & 0 & 3.510159 & -3.568750 & -0.588620 \\
\hline 32 & 1 & $\theta$ & 4.292202 & -1.993146 & -0.587855 \\
\hline 33 & 1 & 0 & 3.446887 & -2.559410 & 0.863802 \\
\hline 34 & 1 & 0 & 0.858371 & -2.614297 & 0.868464 \\
\hline 35 & 6 & 0 & 0.731692 & -4.056425 & -0.737877 \\
\hline 36 & 1 & 0 & -0.195673 & -4.515399 & -0.387151 \\
\hline 37 & 1 & $\odot$ & 0.735212 & -4.083194 & -1.831681 \\
\hline 38 & 1 & 0 & 1.554008 & -4.670940 & -0.364674 \\
\hline
\end{tabular}

\section{Conformer (22f)}

Sum of electronic and thermal Free Energies $=-1185.485567$ Hartrees

$\begin{array}{ccccc}\text { Center } & \text { Atomic } & \text { Atomic } & \text { Coordinates (Angstroms) } \\ \text { Number } & \text { Number } & \text { Type } & X & Y\end{array}$




\begin{tabular}{|c|c|c|c|c|c|}
\hline 2 & 6 & $\odot$ & 2.606877 & -1.159443 & $\odot .159349$ \\
\hline 3 & 1 & $\odot$ & 3.624769 & -1.347696 & -0.202414 \\
\hline 4 & 6 & $\odot$ & 2.436502 & $\odot .328607$ & ๑. 189449 \\
\hline 5 & 1 & $\odot$ & 3.277854 & ๑. 972756 & $\odot .456770$ \\
\hline 6 & 7 & $\odot$ & 1.309402 & ๑. 911318 & -0.029328 \\
\hline 7 & 16 & $\odot$ & 1.142710 & 2.733033 & -0.093198 \\
\hline 8 & 8 & $\odot$ & 2.438760 & 3.203271 & ๑. 365923 \\
\hline 9 & 8 & $\odot$ & 0.610759 & 2.978189 & -1.419794 \\
\hline 10 & 6 & $\odot$ & -0.127269 & 2.968367 & 1.151110 \\
\hline 11 & 1 & $\odot$ & -0.342855 & $4.04 \odot \odot \odot 3$ & 1. 152792 \\
\hline 12 & 1 & $\odot$ & -1.013205 & 2.403243 & 0.854209 \\
\hline 13 & 1 & $\odot$ & 0.263242 & 2.654095 & 2.119385 \\
\hline 14 & 8 & $\odot$ & 0.307528 & -1.410278 & -0.385398 \\
\hline 15 & 6 & $\odot$ & $-\odot .868870$ & -2.126482 & -0.846030 \\
\hline 16 & 1 & $\odot$ & $-\odot .840519$ & -3.142460 & -0.435606 \\
\hline 17 & 1 & $\odot$ & -0.857623 & -2.187004 & -1.939424 \\
\hline 18 & 1 & $\odot$ & 0.518340 & 0.271099 & -0.290627 \\
\hline 19 & 6 & 0 & -2.062987 & -1.354954 & -0.354538 \\
\hline 20 & 6 & $\odot$ & -2.723405 & -1.726666 & 0.822025 \\
\hline 21 & 6 & $\odot$ & -2.486986 & -0.210355 & -1.046533 \\
\hline 22 & 6 & $\odot$ & -3.793697 & -0.969436 & 1.300430 \\
\hline 23 & 1 & $\odot$ & -2.406245 & -2.615960 & 1. 360188 \\
\hline 24 & 6 & $\odot$ & -3.551586 & ๑. 552726 & -0.564488 \\
\hline 25 & 1 & $\odot$ & -1.998802 & $\odot .071040$ & -1.977131 \\
\hline 26 & 6 & $\odot$ & -4.205705 & 0.172372 & $\odot .610879$ \\
\hline 27 & 1 & $\odot$ & -4.308458 & -1.273376 & 2.206400 \\
\hline 28 & 1 & $\odot$ & -3.886431 & 1.425303 & -1.117202 \\
\hline 29 & 1 & $\odot$ & -5.044056 & $\odot .755842$ & 0.978609 \\
\hline 30 & 6 & $\odot$ & 2.528740 & -1.649222 & 1.634718 \\
\hline 31 & 1 & $\odot$ & 2.824714 & -2.700435 & 1.666949 \\
\hline 32 & 1 & $\odot$ & 3.203428 & -1.092486 & 2.290613 \\
\hline 33 & 1 & $\odot$ & 1.506922 & -1.556455 & $2.0 \odot 7821$ \\
\hline 34 & 6 & 0 & 1.905358 & -1.692653 & -2.253944 \\
\hline 35 & 1 & $\odot$ & 1.853744 & -0.637472 & -2.540791 \\
\hline 36 & 1 & $\odot$ & 2.906064 & -2.065419 & -2.492047 \\
\hline 37 & 1 & $\odot$ & 1.199556 & -2.246390 & -2.876949 \\
\hline 38 & 1 & $\odot$ & 1.667954 & -2.967646 & -0.527795 \\
\hline
\end{tabular}

\section{Conformer (21g)}

Sum of electronic and thermal Free Energies $=-1185.485234$ Hartrees

\begin{tabular}{|c|c|c|c|c|c|}
\hline \multirow{2}{*}{$\begin{array}{l}\text { Center } \\
\text { Number }\end{array}$} & \multirow{2}{*}{$\begin{array}{l}\text { Atomic } \\
\text { Number }\end{array}$} & \multirow{2}{*}{$\begin{array}{c}\text { Atomic } \\
\text { Type }\end{array}$} & \multicolumn{3}{|c|}{ Coordinates (Angstroms) } \\
\hline & & & X & $Y$ & z \\
\hline 1 & 6 & $\odot$ & 1.903821 & -3.541364 & -0.573576 \\
\hline 2 & 1 & $\theta$ & 1.769962 & -3.931263 & 0.438238 \\
\hline 3 & 1 & 0 & 2.926256 & -3.753139 & -0.9014 \\
\hline 4 & 1 & $\odot$ & 1.231078 & -4.084227 & -1.241524 \\
\hline 5 & 6 & 0 & 1.622927 & -2.043351 & -0.632392 \\
\hline 6 & 1 & 0 & 1.644332 & -1.716833 & -1.684914 \\
\hline 7 & 6 & 0 & 2.668542 & -1.200562 & 0.14415 \\
\hline 8 & 1 & 0 & 3.642719 & -1.338599 & -0.340696 \\
\hline 9 & 6 & $\theta$ & 2.387221 & 0.270042 & 0.106361 \\
\hline 10 & 1 & $\odot$ & 3.202027 & 0.992665 & 0.195426 \\
\hline 11 & 7 & 0 & 1.195127 & 0.746501 & 0.017078 \\
\hline 12 & 16 & $\theta$ & 0.875078 & 2.541122 & -0.171611 \\
\hline 13 & 8 & 0 & 2.176796 & 3.136882 & 0.078886 \\
\hline
\end{tabular}




$\begin{array}{rrrrrr}14 & 8 & 0 & 0.172020 & 2.631823 & -1.437255 \\ 15 & 6 & 0 & -0.250445 & 2.803069 & 1.198659 \\ 16 & 1 & 0 & -0.515568 & 3.862951 & 1.161520 \\ 17 & 1 & 0 & -1.135536 & 2.183538 & 1.039185 \\ 18 & 1 & 0 & 0.265589 & 2.570382 & 2.130576 \\ 19 & 8 & 0 & 0.340777 & -1.694675 & -0.075823 \\ 20 & 6 & 0 & -0.834982 & -2.183332 & -0.783534 \\ 21 & 1 & 0 & -0.997238 & -3.232534 & -0.519850 \\ 22 & 1 & 0 & -0.654302 & -2.114671 & -1.864029 \\ 23 & 1 & 0 & 0.434565 & 0.025927 & -0.067608 \\ 24 & 6 & 0 & -1.993713 & -1.318792 & -0.366442 \\ 25 & 6 & 0 & -2.783014 & -1.667206 & 0.736687 \\ 26 & 6 & 0 & -2.253712 & -0.117218 & -1.042948 \\ 27 & 6 & 0 & -3.815141 & -0.829288 & 1.159410 \\ 28 & 1 & 0 & -2.592788 & -2.599565 & 1.261303 \\ 29 & 6 & 0 & -3.284293 & 0.724645 & -0.618045 \\ 30 & 1 & 0 & -1.668308 & 0.151943 & -1.919374 \\ 31 & 6 & 0 & -4.064816 & 0.368428 & 0.485296 \\ 32 & 1 & 0 & -4.428633 & -1.113459 & 2.008491 \\ 33 & 1 & 0 & -3.492649 & 1.640338 & -1.163034 \\ 34 & 1 & 0 & -4.876474 & 1.013466 & 0.807335 \\ 35 & 6 & 0 & 2.823933 & -1.590033 & 1.643084 \\ 36 & 1 & 0 & 3.240744 & -2.596113 & 1.711048 \\ 37 & 1 & 0 & 3.508344 & -0.915411 & 2.164944 \\ 38 & 1 & 0 & 1.854044 & -1.571313 & 2.143906 \\ -------------------------1\end{array}$

\section{Conformer (22g)}

Sum of electronic and thermal Free Energies $=-1185.484067$ Hartrees

\begin{tabular}{|c|c|c|c|c|c|}
\hline \multirow{2}{*}{$\begin{array}{l}\text { Center } \\
\text { Number }\end{array}$} & Atomic & Atomic & \multicolumn{3}{|c|}{ Coordinates (Angstroms) } \\
\hline & Number & Type & X & $\mathrm{Y}$ & Z \\
\hline & & & & & $----1---$ \\
\hline 1 & 6 & $\odot$ & -0.731122 & 3.138963 & 1.381392 \\
\hline 2 & 1 & $\odot$ & -1.252918 & 2.334277 & 1.909757 \\
\hline 3 & 1 & $\odot$ & -1.338421 & 4.045160 & 1.445627 \\
\hline 4 & 1 & $\odot$ & $\odot .202135$ & 3.339240 & 1.912561 \\
\hline 5 & 6 & 0 & -0.445357 & 2.767655 & -0.074385 \\
\hline 6 & 1 & $\odot$ & $\odot .024611$ & 3.620668 & -0.582250 \\
\hline 7 & 6 & $\odot$ & -1.712671 & 2.402744 & -0.896215 \\
\hline 8 & 1 & $\odot$ & -1.372423 & 2.256600 & -1.937655 \\
\hline 9 & 6 & $\odot$ & -2.299933 & 1.062168 & -0.569810 \\
\hline 10 & 1 & $\odot$ & -3.355568 & $\odot .867416$ & -0.778319 \\
\hline 11 & 7 & $\odot$ & -1.619116 & ๑.073316 & $-\odot .104087$ \\
\hline 12 & 16 & $\odot$ & -2.339279 & -1.615878 & $\odot .0 \odot \odot 287$ \\
\hline 13 & 8 & 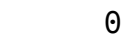 & -3.736531 & -1.395729 & -0.333451 \\
\hline 14 & 8 & $\odot$ & -1.429599 & -2.424755 & -0.785527 \\
\hline 15 & 6 & $\odot$ & -2.133736 & -1.944172 & 1.751481 \\
\hline 16 & 1 & $\odot$ & -2.509693 & -2.958990 & 1.905733 \\
\hline 17 & 1 & $\odot$ & -1.071023 & -1.895568 & 1.993532 \\
\hline 18 & 1 & $\odot$ & -2.726290 & -1.226057 & 2.318845 \\
\hline 19 & 8 & $\odot$ & ๑.424519 & 1.629001 & -0.175486 \\
\hline 20 & 6 & $\odot$ & 1.847960 & 1.864784 & ๑.011177 \\
\hline 21 & 1 & $\odot$ & 2.014674 & 2.362430 & $\odot .972413$ \\
\hline 22 & 1 & $\odot$ & 2.184377 & 2.532415 & -0.791595 \\
\hline 23 & 1 & $\odot$ & -0.595215 & $\odot .251691$ & 0.032029 \\
\hline 24 & 6 & $\odot$ & 2.549000 & 0.536928 & -0.034517 \\
\hline 25 & 6 & $\odot$ & 2.920679 & -0.110326 & 1.150938 \\
\hline 26 & 6 & $\odot$ & 2.808522 & -0.083617 & -1.264364 \\
\hline
\end{tabular}




\begin{tabular}{|c|c|c|c|c|c|}
\hline 27 & 6 & $\odot$ & 3.546623 & -1.356743 & 1.108769 \\
\hline 28 & 1 & $\odot$ & 2.737436 & ๑.371339 & 2.108501 \\
\hline 29 & 6 & $\odot$ & 3.429553 & -1.330693 & -1.307338 \\
\hline 30 & 1 & 0 & 2.532875 & $\odot .416552$ & -2.189182 \\
\hline 31 & 6 & $\odot$ & 3.799276 & -1.968085 & -0.120422 \\
\hline 32 & 1 & $\odot$ & 3.845752 & -1.844232 & 2.031476 \\
\hline 33 & 1 & $\odot$ & 3.634082 & -1.800892 & -2.263957 \\
\hline 34 & 1 & $\odot$ & 4.292145 & -2.934634 & -0.154925 \\
\hline 35 & 6 & $\odot$ & -2.795031 & 3.497235 & -0.901545 \\
\hline 36 & 1 & $\odot$ & -2.355733 & 4.450966 & -1.202958 \\
\hline 37 & 1 & $\odot$ & -3.587875 & 3.258123 & -1.615319 \\
\hline 38 & 1 & $\odot$ & -3.248829 & 3.629185 & 0.083521 \\
\hline
\end{tabular}

\section{REFERENCES}

1. Frisch, M. J.; Trucks, G. W.; Schlegel, H. B.; Scuseria, G. E.; Robb, M. A.; Cheeseman, J. R.; Scalmani, G.; Barone, V.; Petersson, G. A.; Nakatsuji, H.; Li, X.; Caricato, M.; Marenich, A. V.; Bloino, J.; Janesko, B. G.; Gomperts, R.; Mennucci, B.; Hratchian, H. P.; Ortiz, J. V.; Izmaylov, A. F.; Sonnenberg, J. L.; Williams; Ding, F.; Lipparini, F.; Egidi, F.; Goings, J.; Peng, B.; Petrone, A.; Henderson, T.; Ranasinghe, D.; Zakrzewski, V. G.; Gao, J.; Rega, N.; Zheng, G.; Liang, W.; Hada, M.; Ehara, M.; Toyota, K.; Fukuda, R.; Hasegawa, J.; Ishida, M.; Nakajima, T.; Honda, Y.; Kitao, O.; Nakai, H.; Vreven, T.; Throssell, K.; Montgomery Jr., J. A.; Peralta, J. E.; Ogliaro, F.; Bearpark, M. J.; Heyd, J. J.; Brothers, E. N.; Kudin, K. N.; Staroverov, V. N.; Keith, T. A.; Kobayashi, R.; Normand, J.; Raghavachari, K.; Rendell, A. P.; Burant, J. C.; Iyengar, S. S.; Tomasi, J.; Cossi, M.; Millam, J. M.; Klene, M.; Adamo, C.; Cammi, R.; Ochterski, J. W.; Martin, R. L.; Morokuma, K.; Farkas, O.; Foresman, J. B.; Fox, D. J. Gaussian 16 Rev. C.01, Wallingford, CT, 2016.

2. Lee, C.; Yang, W.; Parr, R. G., Physical Review B 1988, 37 (2), 785-789.

3. Becke, A. D., J. Chem. Phys. 1993, 98 (7), 5648-5652. 


\section{X-ray Crystallographic Data}

\section{X-ray Data for compounds anti-17, anti-14e and S7}

X-ray crystallographic data for compound anti-17

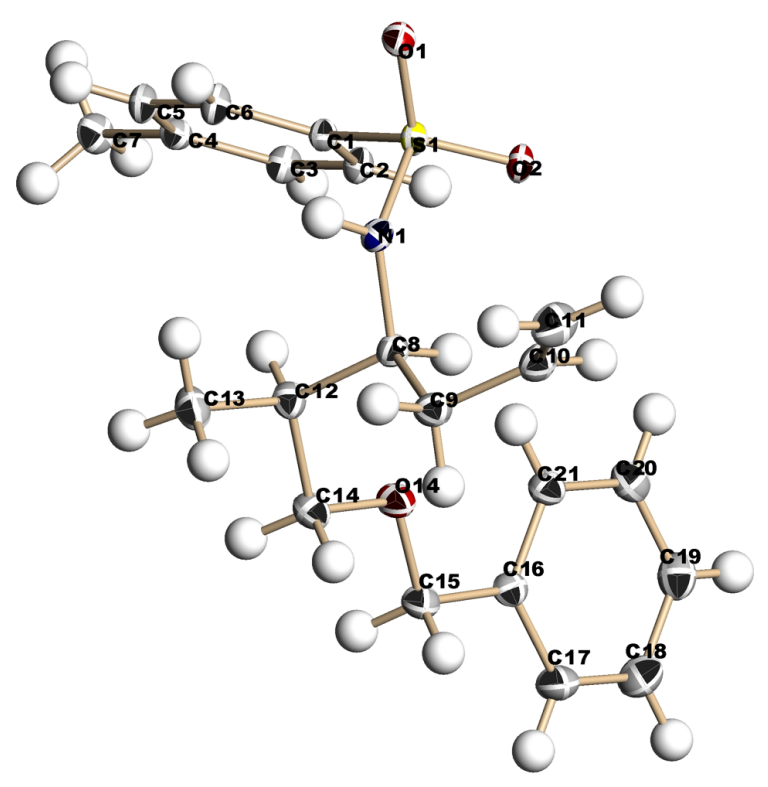

\section{JF2896 Mo}

A colorless plate with approximate orthogonal dimensions $0.096 \times 0.317 \times 0.361 \mathrm{~mm}^{3}$ was placed and optically centered on the Bruker ${ }^{1}$ APEXII CCD system at $-183^{\circ} \mathrm{C}(90 \mathrm{~K})$. Indexing of the unit cell used a random set of reflections collected from three series of $0.5^{\circ}$ wide $\omega$-scans, 10 seconds per frame, and 30 frames per series that were well distributed in reciprocal space. Eight $\omega$-scan data frame series were collected $\left[\mathrm{MoK}_{\alpha}\right]$ with $0.3^{\circ}$ wide scans, 20 seconds per frame and 606 frames collected per series at varying $\phi$ angles. The crystal to detector distance was $5.00 \mathrm{~cm}$, thus providing a complete sphere of data to $2 \theta_{\max }=62.11^{\circ}$.

\section{Structural determination and Refinement:}

All crystallographic calculations were performed on an Intel Xeon E5-1620v2 at 3.70GHz an eight core processor and 16GB of extended memory. Data collected were corrected for Lorentz 
and polarization effects with Saint ${ }^{1}$ and absorption using Blessing's method and merged as incorporated with the program Sadabs ${ }^{2,3}$. The SHELXTL ${ }^{4}$ program package was implemented to determine the probable space group and set up the initial files. System symmetry, lack of systematic absences and intensity statistics indicated the centrosymmetric triclinic space group P-1 (no. 2). The structure was determined by direct methods with the non-hydrogen atoms being located directly for the molecule using the program $\mathrm{XT}^{5}$. The structure was refined with $\mathrm{XL}^{6}$. The 12180 data collected were merged for least squares refinement to 6359 unique data $[\mathrm{R}$ (int) $=0.0157]$. All non-hydrogen atoms were refined anisotropically. Hydrogen atoms were idealized initially and then allowed to refine freely throughout the final refinement. The final structure was refined to convergence with $\mathrm{R}(\mathrm{F})=4.23 \%, \mathrm{wR}\left(\mathrm{F}^{2}\right)=10.08 \%, \mathrm{GOF}=1.020$ for all 6359 unique reflections $\left[R(F)=3.71\right.$, wR $\left(F^{2}\right)=9.66 \%$ for those 5618 data with $\left.F_{0}>4 \sigma(F o)\right]$. The final difference-Fourier map was featureless indicating that the structure is both correct and complete.

Table S1. Crystal data and structure refinement for $\left[\mathrm{C}_{21} \mathrm{H}_{27} \mathrm{NO}_{3} \mathrm{~S}\right]$.

Identification code

Empirical formula

Formula weight

Temperature

Wavelength

Crystal system

Space group

Unit cell dimensions

Volume

Z

Density (calculated)

Absorption coefficient

$\mathrm{F}(000)$

Crystal size

Crystal color and habit

Diffractometer

Theta range for data collection

Index ranges

Reflections collected

Independent reflections

Observed reflections (I > 2sigma(I))
JF2896FMI

$\mathrm{C} 21 \mathrm{H} 27 \mathrm{~N} \mathrm{O} 3 \mathrm{~S}$

373.49

90(2) K

$0.71073 \AA$

Triclinic

P-1

$\mathrm{a}=8.2997(2) \AA \quad \alpha=84.0676(10)^{\circ}$.

$\mathrm{b}=9.2979(2) \AA \quad \beta=81.8080(11)^{\circ}$.

$\mathrm{c}=13.3940(3) \AA \quad \gamma=78.0150(10)^{\circ}$.

997.86(4) $\AA^{3}$

2

$1.243 \mathrm{Mg} / \mathrm{m}^{3}$

$0.182 \mathrm{~mm}^{-1}$

400

$0.361 \times 0.317 \times 0.096 \mathrm{~mm}^{3}$

Colorless Plate

Bruker APEX-II CCD

1.540 to $31.084^{\circ}$.

$-12<=\mathrm{h}<=12,-13<=\mathrm{k}<=13,-19<=\mathrm{l}<=19$

12180

$6359[\mathrm{R}(\mathrm{int})=0.0157]$

5618 
Completeness to theta $=25.242^{\circ}$

Absorption correction

Max. and min. transmission

Solution method

Refinement method

Data / restraints / parameters

Goodness-of-fit on $\mathrm{F}^{2}$

Final R indices [I $>2 \operatorname{sigma}(\mathrm{I})]$

$\mathrm{R}$ indices (all data)

Largest diff. peak and hole
$100.0 \%$

Semi-empirical from equivalents

0.9668 and 0.8960

SHELXT (Sheldrick, 2014)

SHELXL-2018/3 (Sheldrick, 2018) Full-matrix least-squares on $F^{2}$ $6359 / 0 / 343$

1.020

$\mathrm{R} 1=0.0371, \mathrm{wR} 2=0.0966$
$\mathrm{R} 1=0.0423, \mathrm{wR} 2=0.1008$
0.818 and $-0.349 \mathrm{e} . \AA^{-3}$

Table S2. Atomic coordinates ( x 104) and equivalent isotropic displacement parameters $\left(\AA^{2} \mathrm{x}\right.$ $10^{3}$ ) for JF2896FMI. U(eq) is defined as one third of the trace of the orthogonalized $\mathrm{U}^{\mathrm{ij}}$ tensor.

\begin{tabular}{|c|c|c|c|c|}
\hline & $\mathrm{x}$ & $\mathrm{y}$ & $\mathrm{z}$ & $\mathrm{U}(\mathrm{eq})$ \\
\hline $\mathrm{S}(1)$ & $6504(1)$ & $7822(1)$ & $546(1)$ & $15(1)$ \\
\hline $\mathrm{O}(1)$ & $6345(1)$ & $8538(1)$ & $-458(1)$ & $19(1)$ \\
\hline $\mathrm{O}(2)$ & $8047(1)$ & $6869(1)$ & $737(1)$ & $19(1)$ \\
\hline $\mathrm{C}(1)$ & 4931(1) & $6781(1)$ & $854(1)$ & $15(1)$ \\
\hline $\mathrm{N}(1)$ & $6125(1)$ & $9142(1)$ & $1288(1)$ & $17(1)$ \\
\hline$C(2)$ & $5293(1)$ & $5356(1)$ & $1304(1)$ & $17(1)$ \\
\hline$C(3)$ & $4016(1)$ & $4569(1)$ & $1559(1)$ & $19(1)$ \\
\hline$C(4)$ & $2396(1)$ & $5187(1)$ & $1376(1)$ & $17(1)$ \\
\hline$C(5)$ & $2066(1)$ & $6623(1)$ & $920(1)$ & $19(1)$ \\
\hline$C(6)$ & $3320(1)$ & $7426(1)$ & $658(1)$ & $19(1)$ \\
\hline$C(7)$ & 1012(1) & $4342(1)$ & $1660(1)$ & $23(1)$ \\
\hline$C(8)$ & $6355(1)$ & $8875(1)$ & $2365(1)$ & $17(1)$ \\
\hline$C(9)$ & $7173(1)$ & $10106(1)$ & $2632(1)$ & $22(1)$ \\
\hline$C(10)$ & $8819(1)$ & $10123(1)$ & $2002(1)$ & $24(1)$ \\
\hline $\mathrm{C}(11)$ & $9229(2)$ & $11269(2)$ & $1435(1)$ & $32(1)$ \\
\hline$C(12)$ & $4714(1)$ & $8715(1)$ & $3037(1)$ & $20(1)$ \\
\hline$C(13)$ & $3393(2)$ & $10136(1)$ & $3054(1)$ & $27(1)$ \\
\hline$C(14)$ & $5005(1)$ & $8134(1)$ & $4112(1)$ & $22(1)$ \\
\hline $\mathrm{O}(14)$ & $6139(1)$ & $6761(1)$ & 4089(1) & $23(1)$ \\
\hline
\end{tabular}




\begin{tabular}{lllll}
$\mathrm{C}(15)$ & $6472(1)$ & $6175(1)$ & $5072(1)$ & $22(1)$ \\
$\mathrm{C}(16)$ & $7676(1)$ & $4724(1)$ & $5028(1)$ & $19(1)$ \\
$\mathrm{C}(17)$ & $8353(2)$ & $4115(1)$ & $5906(1)$ & $28(1)$ \\
$\mathrm{C}(18)$ & $9471(2)$ & $2782(2)$ & $5913(1)$ & $33(1)$ \\
$\mathrm{C}(19)$ & $9922(2)$ & $2030(1)$ & $5043(1)$ & $28(1)$ \\
$\mathrm{C}(20)$ & $9250(1)$ & $2619(1)$ & $4166(1)$ & $25(1)$ \\
$\mathrm{C}(21)$ & $8129(1)$ & $3967(1)$ & $4154(1)$ & $22(1)$ \\
\hline
\end{tabular}


Table S3. Bond lengths $[\AA]$ and angles $\left[^{\circ}\right]$ for JF2896FMI.

\begin{tabular}{|c|c|c|c|}
\hline $\mathrm{S}(1)-\mathrm{O}(2)$ & $1.4372(7)$ & $\mathrm{C}(13)-\mathrm{H}(13 \mathrm{~B})$ & $0.960(17)$ \\
\hline $\mathrm{S}(1)-\mathrm{O}(1)$ & $1.4485(7)$ & $\mathrm{C}(13)-\mathrm{H}(13 \mathrm{C})$ & $0.983(18)$ \\
\hline $\mathrm{S}(1)-\mathrm{N}(1)$ & $1.6107(9)$ & $\mathrm{C}(14)-\mathrm{O}(14)$ & $1.4208(13)$ \\
\hline$S(1)-C(1)$ & $1.7614(9)$ & $\mathrm{C}(14)-\mathrm{H}(14 \mathrm{~A})$ & $1.001(15)$ \\
\hline $\mathrm{C}(1)-\mathrm{C}(2)$ & $1.3897(13)$ & $\mathrm{C}(14)-\mathrm{H}(14 \mathrm{~B})$ & $1.006(15)$ \\
\hline$C(1)-C(6)$ & $1.3967(13)$ & $\mathrm{O}(14)-\mathrm{C}(15)$ & $1.4139(13)$ \\
\hline $\mathrm{N}(1)-\mathrm{C}(8)$ & $1.4711(13)$ & $C(15)-C(16)$ & $1.5035(15)$ \\
\hline $\mathrm{N}(1)-\mathrm{H}(1)$ & $0.871(18)$ & $\mathrm{C}(15)-\mathrm{H}(15 \mathrm{~A})$ & $0.993(16)$ \\
\hline $\mathrm{C}(2)-\mathrm{C}(3)$ & $1.3935(13)$ & $\mathrm{C}(15)-\mathrm{H}(15 \mathrm{~B})$ & $0.981(16)$ \\
\hline $\mathrm{C}(2)-\mathrm{H}(2)$ & $0.952(15)$ & $C(16)-C(17)$ & $1.3921(15)$ \\
\hline $\mathrm{C}(3)-\mathrm{C}(4)$ & $1.3937(14)$ & $C(16)-C(21)$ & $1.3941(15)$ \\
\hline $\mathrm{C}(3)-\mathrm{H}(3)$ & $0.989(16)$ & $\mathrm{C}(17)-\mathrm{C}(18)$ & $1.3856(18)$ \\
\hline$C(4)-C(5)$ & $1.3990(14)$ & $\mathrm{C}(17)-\mathrm{H}(17)$ & $0.965(18)$ \\
\hline$C(4)-C(7)$ & $1.5069(14)$ & $\mathrm{C}(18)-\mathrm{C}(19)$ & $1.3861(19)$ \\
\hline$C(5)-C(6)$ & $1.3882(13)$ & $\mathrm{C}(18)-\mathrm{H}(18)$ & $0.96(2)$ \\
\hline $\mathrm{C}(5)-\mathrm{H}(5)$ & $0.948(16)$ & $C(19)-C(20)$ & $1.3848(17)$ \\
\hline $\mathrm{C}(6)-\mathrm{H}(6)$ & $0.959(15)$ & $\mathrm{C}(19)-\mathrm{H}(19)$ & $1.004(18)$ \\
\hline $\mathrm{C}(7)-\mathrm{H}(7 \mathrm{~A})$ & $0.949(19)$ & $C(20)-C(21)$ & $1.3974(16)$ \\
\hline $\mathrm{C}(7)-\mathrm{H}(7 \mathrm{~B})$ & $0.97(2)$ & $\mathrm{C}(20)-\mathrm{H}(20)$ & $0.975(17)$ \\
\hline $\mathrm{C}(7)-\mathrm{H}(7 \mathrm{C})$ & $0.959(18)$ & $\mathrm{C}(21)-\mathrm{H}(21)$ & $0.963(17)$ \\
\hline$C(8)-C(9)$ & $1.5409(14)$ & & \\
\hline$C(8)-C(12)$ & $1.5476(14)$ & $\mathrm{O}(2)-\mathrm{S}(1)-\mathrm{O}(1)$ & $119.09(4)$ \\
\hline $\mathrm{C}(8)-\mathrm{H}(8)$ & $0.969(14)$ & $\mathrm{O}(2)-\mathrm{S}(1)-\mathrm{N}(1)$ & $108.63(5)$ \\
\hline $\mathrm{C}(9)-\mathrm{C}(10)$ & $1.5016(15)$ & $\mathrm{O}(1)-\mathrm{S}(1)-\mathrm{N}(1)$ & $105.34(4)$ \\
\hline C(9)-H(9A) & $0.982(16)$ & $\mathrm{O}(2)-\mathrm{S}(1)-\mathrm{C}(1)$ & $107.48(4)$ \\
\hline C(9)-H(9B) & $0.991(17)$ & $\mathrm{O}(1)-\mathrm{S}(1)-\mathrm{C}(1)$ & $107.69(4)$ \\
\hline$C(10)-C(11)$ & $1.3183(17)$ & $\mathrm{N}(1)-\mathrm{S}(1)-\mathrm{C}(1)$ & $108.20(4)$ \\
\hline $\mathrm{C}(10)-\mathrm{H}(10)$ & $0.995(17)$ & $C(2)-C(1)-C(6)$ & $121.06(9)$ \\
\hline $\mathrm{C}(11)-\mathrm{H}(11 \mathrm{~A})$ & $0.98(2)$ & $C(2)-C(1)-S(1)$ & $120.09(7)$ \\
\hline $\mathrm{C}(11)-\mathrm{H}(11 \mathrm{~B})$ & $0.94(2)$ & $C(6)-C(1)-S(1)$ & $118.83(7)$ \\
\hline$C(12)-C(14)$ & $1.5194(15)$ & $\mathrm{C}(8)-\mathrm{N}(1)-\mathrm{S}(1)$ & $121.97(7)$ \\
\hline$C(12)-C(13)$ & $1.5316(15)$ & $\mathrm{C}(8)-\mathrm{N}(1)-\mathrm{H}(1)$ & $118.1(11)$ \\
\hline $\mathrm{C}(12)-\mathrm{H}(12)$ & $1.031(15)$ & $\mathrm{S}(1)-\mathrm{N}(1)-\mathrm{H}(1)$ & $114.2(12)$ \\
\hline $\mathrm{C}(13)-\mathrm{H}(13 \mathrm{~A})$ & $0.985(18)$ & $\mathrm{C}(1)-\mathrm{C}(2)-\mathrm{C}(3)$ & 118.83(9) \\
\hline
\end{tabular}




\begin{tabular}{|c|c|c|c|}
\hline $\mathrm{C}(1)-\mathrm{C}(2)-\mathrm{H}(2)$ & 119.7(9) & $\mathrm{C}(10)-\mathrm{C}(11)-\mathrm{H}(11 \mathrm{~B})$ & $119.9(12)$ \\
\hline $\mathrm{C}(3)-\mathrm{C}(2)-\mathrm{H}(2)$ & $121.4(9)$ & $\mathrm{H}(11 \mathrm{~A})-\mathrm{C}(11)-\mathrm{H}(11 \mathrm{~B})$ & $121.4(16)$ \\
\hline$C(2)-C(3)-C(4)$ & 121.32(9) & $\mathrm{C}(14)-\mathrm{C}(12)-\mathrm{C}(13)$ & 109.67(9) \\
\hline $\mathrm{C}(2)-\mathrm{C}(3)-\mathrm{H}(3)$ & $119.4(9)$ & $\mathrm{C}(14)-\mathrm{C}(12)-\mathrm{C}(8)$ & $111.78(8)$ \\
\hline $\mathrm{C}(4)-\mathrm{C}(3)-\mathrm{H}(3)$ & $119.3(9)$ & $\mathrm{C}(13)-\mathrm{C}(12)-\mathrm{C}(8)$ & $113.76(9)$ \\
\hline$C(3)-C(4)-C(5)$ & $118.70(9)$ & $\mathrm{C}(14)-\mathrm{C}(12)-\mathrm{H}(12)$ & $106.1(8)$ \\
\hline$C(3)-C(4)-C(7)$ & $121.30(9)$ & $\mathrm{C}(13)-\mathrm{C}(12)-\mathrm{H}(12)$ & $107.3(8)$ \\
\hline$C(5)-C(4)-C(7)$ & $120.00(9)$ & $\mathrm{C}(8)-\mathrm{C}(12)-\mathrm{H}(12)$ & $107.8(8)$ \\
\hline$C(6)-C(5)-C(4)$ & $120.95(9)$ & $\mathrm{C}(12)-\mathrm{C}(13)-\mathrm{H}(13 \mathrm{~A})$ & $108.8(10)$ \\
\hline $\mathrm{C}(6)-\mathrm{C}(5)-\mathrm{H}(5)$ & $120.4(9)$ & $\mathrm{C}(12)-\mathrm{C}(13)-\mathrm{H}(13 \mathrm{~B})$ & $112.5(10)$ \\
\hline $\mathrm{C}(4)-\mathrm{C}(5)-\mathrm{H}(5)$ & $118.6(9)$ & $\mathrm{H}(13 \mathrm{~A})-\mathrm{C}(13)-\mathrm{H}(13 \mathrm{~B})$ & $107.8(14)$ \\
\hline$C(5)-C(6)-C(1)$ & $119.15(9)$ & $\mathrm{C}(12)-\mathrm{C}(13)-\mathrm{H}(13 \mathrm{C})$ & $111.9(10)$ \\
\hline $\mathrm{C}(5)-\mathrm{C}(6)-\mathrm{H}(6)$ & $118.8(9)$ & $\mathrm{H}(13 \mathrm{~A})-\mathrm{C}(13)-\mathrm{H}(13 \mathrm{C})$ & $108.5(14)$ \\
\hline $\mathrm{C}(1)-\mathrm{C}(6)-\mathrm{H}(6)$ & $122.1(9)$ & $\mathrm{H}(13 \mathrm{~B})-\mathrm{C}(13)-\mathrm{H}(13 \mathrm{C})$ & $107.2(14)$ \\
\hline $\mathrm{C}(4)-\mathrm{C}(7)-\mathrm{H}(7 \mathrm{~A})$ & $111.5(11)$ & $\mathrm{O}(14)-\mathrm{C}(14)-\mathrm{C}(12)$ & $109.09(8)$ \\
\hline $\mathrm{C}(4)-\mathrm{C}(7)-\mathrm{H}(7 \mathrm{~B})$ & $111.4(11)$ & $\mathrm{O}(14)-\mathrm{C}(14)-\mathrm{H}(14 \mathrm{~A})$ & $109.9(8)$ \\
\hline $\mathrm{H}(7 \mathrm{~A})-\mathrm{C}(7)-\mathrm{H}(7 \mathrm{~B})$ & $107.7(16)$ & $\mathrm{C}(12)-\mathrm{C}(14)-\mathrm{H}(14 \mathrm{~A})$ & $109.5(8)$ \\
\hline $\mathrm{C}(4)-\mathrm{C}(7)-\mathrm{H}(7 \mathrm{C})$ & $110.4(10)$ & $\mathrm{O}(14)-\mathrm{C}(14)-\mathrm{H}(14 \mathrm{~B})$ & $109.8(9)$ \\
\hline $\mathrm{H}(7 \mathrm{~A})-\mathrm{C}(7)-\mathrm{H}(7 \mathrm{C})$ & $110.4(15)$ & $\mathrm{C}(12)-\mathrm{C}(14)-\mathrm{H}(14 \mathrm{~B})$ & 111.1(9) \\
\hline $\mathrm{H}(7 \mathrm{~B})-\mathrm{C}(7)-\mathrm{H}(7 \mathrm{C})$ & $105.3(15)$ & $\mathrm{H}(14 \mathrm{~A})-\mathrm{C}(14)-\mathrm{H}(14 \mathrm{~B})$ & $107.4(12)$ \\
\hline $\mathrm{N}(1)-\mathrm{C}(8)-\mathrm{C}(9)$ & $107.55(8)$ & $\mathrm{C}(15)-\mathrm{O}(14)-\mathrm{C}(14)$ & $111.59(8)$ \\
\hline $\mathrm{N}(1)-\mathrm{C}(8)-\mathrm{C}(12)$ & $111.61(8)$ & $\mathrm{O}(14)-\mathrm{C}(15)-\mathrm{C}(16)$ & $110.61(8)$ \\
\hline $\mathrm{C}(9)-\mathrm{C}(8)-\mathrm{C}(12)$ & $114.79(9)$ & $\mathrm{O}(14)-\mathrm{C}(15)-\mathrm{H}(15 \mathrm{~A})$ & $109.3(9)$ \\
\hline $\mathrm{N}(1)-\mathrm{C}(8)-\mathrm{H}(8)$ & $103.3(8)$ & $\mathrm{C}(16)-\mathrm{C}(15)-\mathrm{H}(15 \mathrm{~A})$ & $110.3(9)$ \\
\hline $\mathrm{C}(9)-\mathrm{C}(8)-\mathrm{H}(8)$ & $110.2(8)$ & $\mathrm{O}(14)-\mathrm{C}(15)-\mathrm{H}(15 \mathrm{~B})$ & $108.5(9)$ \\
\hline $\mathrm{C}(12)-\mathrm{C}(8)-\mathrm{H}(8)$ & $108.8(8)$ & $\mathrm{C}(16)-\mathrm{C}(15)-\mathrm{H}(15 \mathrm{~B})$ & $110.2(9)$ \\
\hline$C(10)-C(9)-C(8)$ & $111.81(9)$ & $\mathrm{H}(15 \mathrm{~A})-\mathrm{C}(15)-\mathrm{H}(15 \mathrm{~B})$ & $107.8(13)$ \\
\hline $\mathrm{C}(10)-\mathrm{C}(9)-\mathrm{H}(9 \mathrm{~A})$ & $108.9(9)$ & $\mathrm{C}(17)-\mathrm{C}(16)-\mathrm{C}(21)$ & $119.01(10)$ \\
\hline $\mathrm{C}(8)-\mathrm{C}(9)-\mathrm{H}(9 \mathrm{~A})$ & $111.7(10)$ & $C(17)-C(16)-C(15)$ & $118.02(10)$ \\
\hline $\mathrm{C}(10)-\mathrm{C}(9)-\mathrm{H}(9 \mathrm{~B})$ & $110.7(10)$ & $\mathrm{C}(21)-\mathrm{C}(16)-\mathrm{C}(15)$ & $122.97(9)$ \\
\hline $\mathrm{C}(8)-\mathrm{C}(9)-\mathrm{H}(9 \mathrm{~B})$ & $108.4(10)$ & $\mathrm{C}(18)-\mathrm{C}(17)-\mathrm{C}(16)$ & $120.72(11)$ \\
\hline $\mathrm{H}(9 \mathrm{~A})-\mathrm{C}(9)-\mathrm{H}(9 \mathrm{~B})$ & $105.0(13)$ & $\mathrm{C}(18)-\mathrm{C}(17)-\mathrm{H}(17)$ & $122.0(10)$ \\
\hline C(11)-C(10)-C(9) & $125.28(12)$ & $\mathrm{C}(16)-\mathrm{C}(17)-\mathrm{H}(17)$ & $117.2(10)$ \\
\hline $\mathrm{C}(11)-\mathrm{C}(10)-\mathrm{H}(10)$ & $118.9(10)$ & $\mathrm{C}(17)-\mathrm{C}(18)-\mathrm{C}(19)$ & $120.24(11)$ \\
\hline $\mathrm{C}(9)-\mathrm{C}(10)-\mathrm{H}(10)$ & $115.7(10)$ & $\mathrm{C}(17)-\mathrm{C}(18)-\mathrm{H}(18)$ & $118.2(13)$ \\
\hline $\mathrm{C}(10)-\mathrm{C}(11)-\mathrm{H}(11 \mathrm{~A})$ & $118.7(12)$ & $\mathrm{C}(19)-\mathrm{C}(18)-\mathrm{H}(18)$ & $121.5(12)$ \\
\hline
\end{tabular}




$\begin{array}{llll}\mathrm{C}(20)-\mathrm{C}(19)-\mathrm{C}(18) & 119.65(11) & \mathrm{C}(21)-\mathrm{C}(20)-\mathrm{H}(20) & 119.1(10) \\ \mathrm{C}(20)-\mathrm{C}(19)-\mathrm{H}(19) & 120.1(10) & \mathrm{C}(16)-\mathrm{C}(21)-\mathrm{C}(20) & 120.05(10) \\ \mathrm{C}(18)-\mathrm{C}(19)-\mathrm{H}(19) & 120.2(10) & \mathrm{C}(16)-\mathrm{C}(21)-\mathrm{H}(21) & 117.8(10) \\ \mathrm{C}(19)-\mathrm{C}(20)-\mathrm{C}(21) & 120.33(11) & \mathrm{C}(20)-\mathrm{C}(21)-\mathrm{H}(21) & 122.2(10) \\ \mathrm{C}(19)-\mathrm{C}(20)-\mathrm{H}(20) & 120.6(10) & & \end{array}$

Symmetry transformations used to generate equivalent atoms:

Table S4. Anisotropic displacement parameters $\left(\AA^{2} \times 10^{3}\right)$ for JF2896FMI. The anisotropic displacement factor exponent takes the form: $-2 \pi^{2}\left[h^{2} a^{* 2} U^{11}+\ldots+2 h k a * b * U^{12}\right]$

\begin{tabular}{|c|c|c|c|c|c|c|}
\hline & $\mathrm{U}^{11}$ & $\mathrm{U}^{22}$ & $\mathrm{U}^{33}$ & $\mathrm{U}^{23}$ & $\mathrm{U}^{13}$ & $\mathrm{U}^{12}$ \\
\hline$S(1)$ & $11(1)$ & $15(1)$ & $17(1)$ & $0(1)$ & $-1(1)$ & $-3(1)$ \\
\hline $\mathrm{O}(1)$ & $17(1)$ & $21(1)$ & $17(1)$ & $2(1)$ & $-2(1)$ & $-4(1)$ \\
\hline $\mathrm{O}(2)$ & 11(1) & $21(1)$ & $24(1)$ & $1(1)$ & $-4(1)$ & $-2(1)$ \\
\hline$C(1)$ & $13(1)$ & $15(1)$ & $16(1)$ & $-1(1)$ & $-2(1)$ & $-4(1)$ \\
\hline $\mathrm{N}(1)$ & $17(1)$ & $16(1)$ & $18(1)$ & $0(1)$ & $-4(1)$ & $-3(1)$ \\
\hline$C(2)$ & $15(1)$ & $16(1)$ & 21(1) & $0(1)$ & $-4(1)$ & $-2(1)$ \\
\hline$C(3)$ & $20(1)$ & $16(1)$ & 21(1) & $2(1)$ & $-3(1)$ & $-5(1)$ \\
\hline$C(4)$ & $17(1)$ & $18(1)$ & $17(1)$ & $-3(1)$ & $0(1)$ & $-6(1)$ \\
\hline$C(5)$ & $13(1)$ & $20(1)$ & $24(1)$ & $1(1)$ & $-3(1)$ & $-4(1)$ \\
\hline$C(6)$ & $14(1)$ & $17(1)$ & $25(1)$ & $3(1)$ & $-4(1)$ & $-4(1)$ \\
\hline$C(7)$ & $21(1)$ & $24(1)$ & $26(1)$ & $-1(1)$ & $1(1)$ & $-11(1)$ \\
\hline$C(8)$ & $16(1)$ & $19(1)$ & $16(1)$ & $1(1)$ & $-3(1)$ & $-5(1)$ \\
\hline$C(9)$ & $22(1)$ & $24(1)$ & $20(1)$ & $-3(1)$ & $-3(1)$ & $-8(1)$ \\
\hline$C(10)$ & $22(1)$ & $29(1)$ & $24(1)$ & $-1(1)$ & $-6(1)$ & $-10(1)$ \\
\hline $\mathrm{C}(11)$ & $32(1)$ & $39(1)$ & $31(1)$ & $4(1)$ & $-6(1)$ & $-20(1)$ \\
\hline$C(12)$ & $18(1)$ & $20(1)$ & $20(1)$ & $1(1)$ & $0(1)$ & $-4(1)$ \\
\hline$C(13)$ & $21(1)$ & $26(1)$ & $28(1)$ & $2(1)$ & $2(1)$ & $1(1)$ \\
\hline$C(14)$ & $23(1)$ & $20(1)$ & $20(1)$ & $-1(1)$ & 1(1) & $-3(1)$ \\
\hline $\mathrm{O}(14)$ & $26(1)$ & $23(1)$ & $17(1)$ & $1(1)$ & $-2(1)$ & $-1(1)$ \\
\hline$C(15)$ & $23(1)$ & $24(1)$ & $17(1)$ & $0(1)$ & $-2(1)$ & $-4(1)$ \\
\hline$C(16)$ & $19(1)$ & $22(1)$ & $18(1)$ & $0(1)$ & $-1(1)$ & $-7(1)$ \\
\hline $\mathrm{C}(17)$ & $31(1)$ & $32(1)$ & $20(1)$ & $-2(1)$ & $-6(1)$ & $-3(1)$ \\
\hline $\mathrm{C}(18)$ & $34(1)$ & $36(1)$ & $28(1)$ & $4(1)$ & $-10(1)$ & $-1(1)$ \\
\hline
\end{tabular}




$\begin{array}{lllllll}\mathrm{C}(19) & 24(1) & 25(1) & 34(1) & 3(1) & -3(1) & -3(1) \\ \mathrm{C}(20) & 24(1) & 25(1) & 26(1) & -3(1) & 1(1) & -6(1) \\ \mathrm{C}(21) & 22(1) & 26(1) & 18(1) & 0(1) & -1(1) & -6(1)\end{array}$

Table S5. Hydrogen coordinates ( $\left.\times 10^{4}\right)$ and isotropic displacement parameters $\left(\AA^{2} \times 10^{3}\right)$ for JF2896FMI.

\begin{tabular}{|c|c|c|c|c|}
\hline & $\mathrm{x}$ & $\mathrm{y}$ & z & $\mathrm{U}(\mathrm{eq})$ \\
\hline $\mathrm{H}(1)$ & $5380(20)$ & $9895(19)$ & $1108(13)$ & $38(4)$ \\
\hline $\mathrm{H}(2)$ & $6407(19)$ & $4921(16)$ & $1405(11)$ & $26(4)$ \\
\hline $\mathrm{H}(3)$ & $4270(20)$ & $3548(17)$ & $1869(12)$ & $31(4)$ \\
\hline $\mathrm{H}(5)$ & $970(20)$ & $7037(17)$ & $787(12)$ & $29(4)$ \\
\hline $\mathrm{H}(6)$ & $3053(19)$ & $8403(17)$ & $340(12)$ & $28(4)$ \\
\hline $\mathrm{H}(7 \mathrm{~A})$ & $1410(20)$ & $3380(20)$ & 1948(14) & $46(5)$ \\
\hline $\mathrm{H}(7 \mathrm{~B})$ & $480(20)$ & $4240(20)$ & $1075(15)$ & $47(5)$ \\
\hline $\mathrm{H}(7 \mathrm{C})$ & $150(20)$ & $4867(19)$ & $2118(13)$ & $39(5)$ \\
\hline $\mathrm{H}(8)$ & $7122(17)$ & $7936(15)$ & 2394(10) & $18(3)$ \\
\hline $\mathrm{H}(9 \mathrm{~A})$ & $6460(20)$ & $11082(18)$ & $2549(12)$ & $32(4)$ \\
\hline $\mathrm{H}(9 \mathrm{~B})$ & $7300(20)$ & $9958(18)$ & $3362(13)$ & $33(4)$ \\
\hline $\mathrm{H}(10)$ & $9620(20)$ & 9168(19) & $2000(13)$ & $37(4)$ \\
\hline $\mathrm{H}(11 \mathrm{~A})$ & $8410(30)$ & $12190(20)$ & $1405(16)$ & $56(6)$ \\
\hline $\mathrm{H}(11 \mathrm{~B})$ & $10310(20)$ & $11200(20)$ & $1085(14)$ & $51(5)$ \\
\hline $\mathrm{H}(12)$ & $4231(18)$ & $7932(16)$ & $2749(11)$ & 21(3) \\
\hline $\mathrm{H}(13 \mathrm{~A})$ & $2340(20)$ & 9912(19) & $3418(13)$ & $38(4)$ \\
\hline $\mathrm{H}(13 \mathrm{~B})$ & $3180(20)$ & $10552(19)$ & $2388(13)$ & $39(4)$ \\
\hline $\mathrm{H}(13 \mathrm{C})$ & $3710(20)$ & 10898(19) & $3397(13)$ & $38(4)$ \\
\hline $\mathrm{H}(14 \mathrm{~A})$ & $5470(18)$ & $8859(16)$ & $4432(11)$ & 21(3) \\
\hline $\mathrm{H}(14 \mathrm{~B})$ & $3934(19)$ & $8008(17)$ & $4537(11)$ & $27(4)$ \\
\hline $\mathrm{H}(15 \mathrm{~A})$ & $5420(20)$ & $6048(17)$ & $5492(12)$ & $28(4)$ \\
\hline $\mathrm{H}(15 \mathrm{~B})$ & 6932(19) & $6886(17)$ & $5382(12)$ & $28(4)$ \\
\hline $\mathrm{H}(17)$ & $8050(20)$ & 4684(19) & $6492(14)$ & $40(5)$ \\
\hline $\mathrm{H}(18)$ & $9940(30)$ & $2410(20)$ & $6530(16)$ & $58(6)$ \\
\hline H(19) & $10740(20)$ & $1070(20)$ & $5047(13)$ & $41(5)$ \\
\hline
\end{tabular}


$\mathrm{H}(20)$

9560(20)

2107(18)

3547(13)

36(4)

$\mathrm{H}(21)$

$7640(20)$

4404(19)

3555(13)

37(4)

Table S6. Torsion angles $\left[^{\circ}\right]$ for JF2896FMI.

\begin{tabular}{lclc}
\hline $\mathrm{O}(2)-\mathrm{S}(1)-\mathrm{C}(1)-\mathrm{C}(2)$ & $6.39(9)$ & $\mathrm{N}(1)-\mathrm{C}(8)-\mathrm{C}(9)-\mathrm{C}(10)$ & $-59.40(11)$ \\
$\mathrm{O}(1)-\mathrm{S}(1)-\mathrm{C}(1)-\mathrm{C}(2)$ & $135.85(8)$ & $\mathrm{C}(12)-\mathrm{C}(8)-\mathrm{C}(9)-\mathrm{C}(10)$ & $175.74(9)$ \\
$\mathrm{N}(1)-\mathrm{S}(1)-\mathrm{C}(1)-\mathrm{C}(2)$ & $-110.75(8)$ & $\mathrm{C}(8)-\mathrm{C}(9)-\mathrm{C}(10)-\mathrm{C}(11)$ & $124.30(13)$ \\
$\mathrm{O}(2)-\mathrm{S}(1)-\mathrm{C}(1)-\mathrm{C}(6)$ & $-174.99(8)$ & $\mathrm{N}(1)-\mathrm{C}(8)-\mathrm{C}(12)-\mathrm{C}(14)$ & $168.37(8)$ \\
$\mathrm{O}(1)-\mathrm{S}(1)-\mathrm{C}(1)-\mathrm{C}(6)$ & $-45.54(9)$ & $\mathrm{C}(9)-\mathrm{C}(8)-\mathrm{C}(12)-\mathrm{C}(14)$ & $-68.93(11)$ \\
$\mathrm{N}(1)-\mathrm{S}(1)-\mathrm{C}(1)-\mathrm{C}(6)$ & $67.86(9)$ & $\mathrm{N}(1)-\mathrm{C}(8)-\mathrm{C}(12)-\mathrm{C}(13)$ & $-66.74(12)$ \\
$\mathrm{O}(2)-\mathrm{S}(1)-\mathrm{N}(1)-\mathrm{C}(8)$ & $-43.99(9)$ & $\mathrm{C}(9)-\mathrm{C}(8)-\mathrm{C}(12)-\mathrm{C}(13)$ & $55.96(12)$ \\
$\mathrm{O}(1)-\mathrm{S}(1)-\mathrm{N}(1)-\mathrm{C}(8)$ & $-172.64(7)$ & $\mathrm{C}(13)-\mathrm{C}(12)-\mathrm{C}(14)-\mathrm{O}(14)$ & $175.67(9)$ \\
$\mathrm{C}(1)-\mathrm{S}(1)-\mathrm{N}(1)-\mathrm{C}(8)$ & $72.41(8)$ & $\mathrm{C}(8)-\mathrm{C}(12)-\mathrm{C}(14)-\mathrm{O}(14)$ & $-57.20(11)$ \\
$\mathrm{C}(6)-\mathrm{C}(1)-\mathrm{C}(2)-\mathrm{C}(3)$ & $0.06(15)$ & $\mathrm{C}(12)-\mathrm{C}(14)-\mathrm{O}(14)-\mathrm{C}(15)$ & $179.30(8)$ \\
$\mathrm{S}(1)-\mathrm{C}(1)-\mathrm{C}(2)-\mathrm{C}(3)$ & $178.64(8)$ & $\mathrm{C}(14)-\mathrm{O}(14)-\mathrm{C}(15)-\mathrm{C}(16)$ & $-179.89(9)$ \\
$\mathrm{C}(1)-\mathrm{C}(2)-\mathrm{C}(3)-\mathrm{C}(4)$ & $-0.32(15)$ & $\mathrm{O}(14)-\mathrm{C}(15)-\mathrm{C}(16)-\mathrm{C}(17)$ & $169.49(10)$ \\
$\mathrm{C}(2)-\mathrm{C}(3)-\mathrm{C}(4)-\mathrm{C}(5)$ & $0.45(15)$ & $\mathrm{O}(14)-\mathrm{C}(15)-\mathrm{C}(16)-\mathrm{C}(21)$ & $-10.96(14)$ \\
$\mathrm{C}(2)-\mathrm{C}(3)-\mathrm{C}(4)-\mathrm{C}(7)$ & $-179.46(10)$ & $\mathrm{C}(21)-\mathrm{C}(16)-\mathrm{C}(17)-\mathrm{C}(18)$ & $0.53(18)$ \\
$\mathrm{C}(3)-\mathrm{C}(4)-\mathrm{C}(5)-\mathrm{C}(6)$ & $-0.31(15)$ & $\mathrm{C}(15)-\mathrm{C}(16)-\mathrm{C}(17)-\mathrm{C}(18)$ & $-179.91(11)$ \\
$\mathrm{C}(7)-\mathrm{C}(4)-\mathrm{C}(5)-\mathrm{C}(6)$ & $179.59(10)$ & $\mathrm{C}(16)-\mathrm{C}(17)-\mathrm{C}(18)-\mathrm{C}(19)$ & $-0.5(2)$ \\
$\mathrm{C}(4)-\mathrm{C}(5)-\mathrm{C}(6)-\mathrm{C}(1)$ & $0.06(16)$ & $\mathrm{C}(17)-\mathrm{C}(18)-\mathrm{C}(19)-\mathrm{C}(20)$ & $0.0(2)$ \\
$\mathrm{C}(2)-\mathrm{C}(1)-\mathrm{C}(6)-\mathrm{C}(5)$ & $0.08(15)$ & $\mathrm{C}(18)-\mathrm{C}(19)-\mathrm{C}(20)-\mathrm{C}(21)$ & $0.33(18)$ \\
$\mathrm{S}(1)-\mathrm{C}(1)-\mathrm{C}(6)-\mathrm{C}(5)$ & $-178.53(8)$ & $\mathrm{C}(17)-\mathrm{C}(16)-\mathrm{C}(21)-\mathrm{C}(20)$ & $-0.16(16)$ \\
$\mathrm{S}(1)-\mathrm{N}(1)-\mathrm{C}(8)-\mathrm{C}(9)$ & $137.92(8)$ & $\mathrm{C}(15)-\mathrm{C}(16)-\mathrm{C}(21)-\mathrm{C}(20)$ & $-179.70(10)$ \\
$\mathrm{S}(1)-\mathrm{N}(1)-\mathrm{C}(8)-\mathrm{C}(12)$ & $-95.33(9)$ & $\mathrm{C}(19)-\mathrm{C}(20)-\mathrm{C}(21)-\mathrm{C}(16)$ & $-0.27(17)$ \\
& & &
\end{tabular}

Symmetry transformations used to generate equivalent atoms:

Table S7. Hydrogen bonds for JF2896FMI [ $\AA$ and $\left.{ }^{\circ}\right]$.

\begin{tabular}{lcccc}
\hline $\mathrm{D}-\mathrm{H} \ldots \mathrm{A}$ & $\mathrm{d}(\mathrm{D}-\mathrm{H})$ & $\mathrm{d}(\mathrm{H} \ldots \mathrm{A})$ & $\mathrm{d}(\mathrm{D} \ldots \mathrm{A})$ & $<(\mathrm{DHA})$ \\
\hline $\mathrm{N}(1)-\mathrm{H}(1) \ldots \mathrm{O}(1) \# 1$ & $0.871(18)$ & $2.038(18)$ & $2.8996(11)$ & $169.6(16)$ \\
$\mathrm{C}(8)-\mathrm{H}(8) \ldots \mathrm{O}(2)$ & $0.969(14)$ & $2.480(14)$ & $3.0137(13)$ & $114.5(10)$
\end{tabular}


Symmetry transformations used to generate equivalent atoms:

$\# 1-x+1,-y+2,-z$

X-ray crystallographic data for compound anti-21f

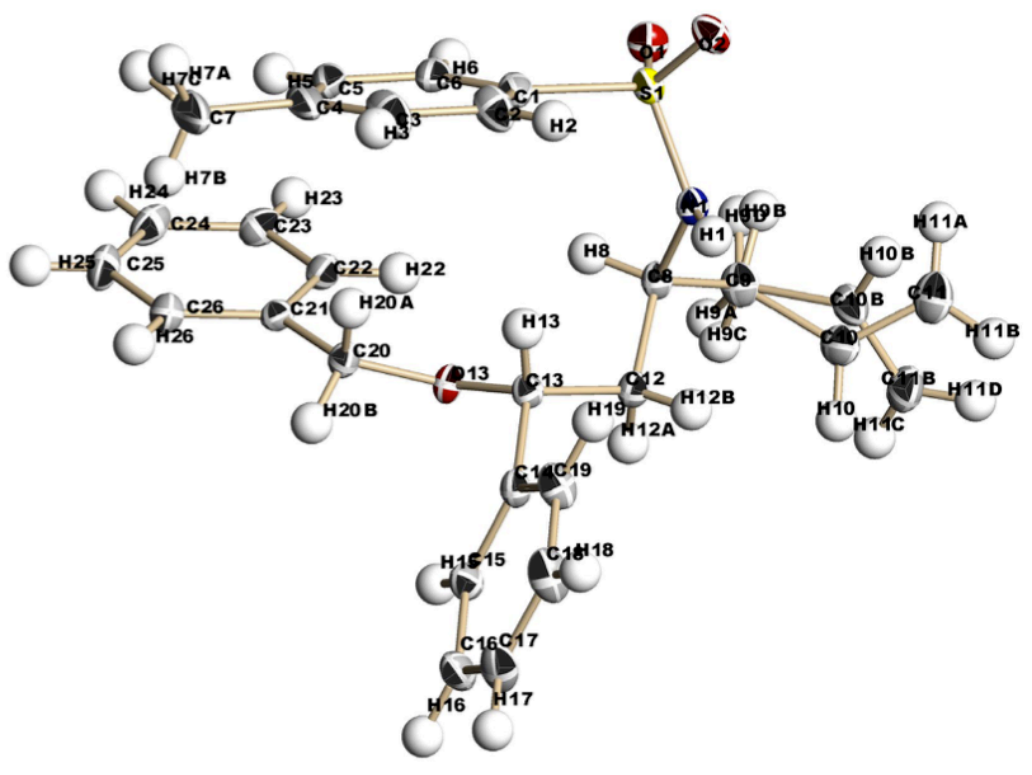

\section{JF3035 Mo}

A long colorless plate with approximate orthogonal dimensions $0.110 \times 0.417 \times 0.962 \mathrm{~mm} 3$ was placed and optically centered on the Bruker Duo(1) APEXII CCD system at ñ1830C(90K). Indexing of the unit cell used a random set of reflections collected from three series of $0.5 \infty$ wide omega-scans, 10 seconds per frame, and 30 frames per series that were well distributed in reciprocal space. Five omega-scan data frame series were collected [MoKa] with $0.3 \infty$ wide scans, 20 seconds per frame and 606 frames collected per series at varying $\mathrm{f}$ angles (phi $=0 \infty$, $72 \infty, 144 \infty, 216 \infty, 288 \infty)$. The crystal to detector distance was $5.15 \mathrm{~cm}$, thus providing a complete sphere of data to 2 thetamax $=61.34 \infty$.

Structural determination and Refinement:

All crystallographic calculations were performed on a Surface Pro7 with Intel i7-1065G7 at $1.30 \mathrm{GHz}$ with four cores, eight processors and $16 \mathrm{~GB}$ of extended memory. Data collected were corrected for Lorentz and polarization effects with Saint1 and absorption using Blessingís method and merged as incorporated with the program Sadabs(2,3). The SHELXTL(4) program package was implemented to determine the probable space group and set up the initial files. System symmetry, systematic absences and intensity statistics indicated the non-standard 
centrosymmetric monoclinic space group P21/n (no. 14). The structure was determined by direct methods with nearly all non-hydrogen atoms being located directly for the molecule using the program XT(5). The structure was refined with XL(6). The 48064 data collected were merged, based upon identical indices to 28376 data, then truncated to 21214 and then merged for least squares refinement to 5397 unique data $[\mathrm{R}(\mathrm{int})=0.0220]$. All non-hydrogen atoms were refined anisotropically. All hydrogen atoms were idealized initially and then those fully occupied were allowed to refine freely throughout the final refinement stage. There is significant libration at $\mathrm{C}(7)$, and a clear disorder so this methyl group was idealized as two sets of tetrahedral hydrogen atoms rotated 60 degrees from one another. The final structure was refined to convergence with $\mathrm{R}(\mathrm{F})=4.00 \%, \quad \mathrm{wR}(\mathrm{F} 2)=9.06 \%, \mathrm{GOF}=1.028$ for all 5397 unique reflections $[\mathrm{R}(\mathrm{F})=3.42$, $w R(F 2)=8.69 \%$ for those 4730 data with $F o>4 \operatorname{sigma}(F o)]$. The final difference-Fourier map was featureless indicating that the structure is both correct and complete. An empirical correction for extinction was also attempted but found to be less than 3 sigma and therefore not applied.

Table S8. Crystal data and structure refinement for $\left[\mathrm{C}_{26} \mathrm{H}_{29} \mathrm{NO}_{3} \mathrm{~S}\right]$.

\begin{tabular}{|c|c|c|}
\hline Identification code & \multicolumn{2}{|c|}{ JF3035FMI（DAG-3-172) } \\
\hline Empirical formula & \multicolumn{2}{|l|}{$\mathrm{C} 26 \mathrm{H} 29 \mathrm{~N} \mathrm{O} 3 \mathrm{~S}$} \\
\hline Formula weight & \multicolumn{2}{|l|}{435.56} \\
\hline Temperature & \multicolumn{2}{|l|}{$90(2) \mathrm{K}$} \\
\hline Wavelength & \multicolumn{2}{|l|}{$0.71073 \AA$} \\
\hline Crystal system & \multicolumn{2}{|l|}{ Monoclinic } \\
\hline Space group & \multicolumn{2}{|l|}{$\mathrm{P} 21 / \mathrm{n}$} \\
\hline \multirow[t]{3}{*}{ Unit cell dimensions } & $\mathrm{a}=9.8856(16) \AA$ & $\mathrm{a}=90^{\circ}$ \\
\hline & $\mathrm{b}=26.555(4) \AA$ & $\mathrm{b}=117.166(2)^{\circ}$ \\
\hline & $\mathrm{c}=10.0914(16) \AA$ & $\mathrm{g}=90^{\circ}$ \\
\hline Volume & \multicolumn{2}{|l|}{$2356.8(7) \AA^{3}$} \\
\hline $\mathrm{Z}$ & \multicolumn{2}{|l|}{4} \\
\hline Density (calculated) & \multicolumn{2}{|l|}{$1.228 \mathrm{Mg} / \mathrm{m}^{3}$} \\
\hline Absorption coefficient & \multicolumn{2}{|l|}{$0.164 \mathrm{~mm}^{-1}$} \\
\hline $\mathrm{F}(000)$ & \multicolumn{2}{|l|}{928} \\
\hline Crystal size & \multicolumn{2}{|c|}{$0.962 \times 0.417 \times 0.110 \mathrm{~mm}^{3}$} \\
\hline Crystal color and habit & \multicolumn{2}{|l|}{ Colorless Plate } \\
\hline Diffractometer & \multicolumn{2}{|c|}{ Bruker APEX-II CCD } \\
\hline Theta range for data collection & \multicolumn{2}{|l|}{2.390 to $27.483^{\circ}$. } \\
\hline Index ranges & \multicolumn{2}{|c|}{$-12<=\mathrm{h}<=12,-34<=\mathrm{k}<=34,-13<=\mathrm{l}<=13$} \\
\hline Reflections collected & \multicolumn{2}{|l|}{21214} \\
\hline Independent reflections & \multicolumn{2}{|c|}{$5397[\mathrm{R}(\mathrm{int})=0.0220]$} \\
\hline Observed reflections $(\mathrm{I}>2 \operatorname{sigma}(\mathrm{I}))$ & \multicolumn{2}{|l|}{4730} \\
\hline
\end{tabular}


Completeness to theta $=25.242^{\circ}$

Absorption correction

Max. and min. transmission

Solution method

Refinement method

Data / restraints / parameters

Goodness-of-fit on $\mathrm{F}^{2}$

Final $\mathrm{R}$ indices [I>2sigma(I)]

$\mathrm{R}$ indices (all data)

Largest diff. peak and hole
$100.0 \%$

Semi-empirical from equivalents

0.9420 and 0.8698

SHELXT (Sheldrick, 2014)

SHELXL-2017/1 (Sheldrick, 2017) Full-matrix least-squares on $F^{2}$

$5397 / 8 / 382$

1.028

$$
\begin{aligned}
& \mathrm{R} 1=0.0342, \mathrm{wR} 2=0.0869 \\
& \mathrm{R} 1=0.0400, \mathrm{wR} 2=0.0906 \\
& 0.429 \text { and }-0.369 \mathrm{e} . \AA^{-3}
\end{aligned}
$$

Table S9. Atomic coordinates ( $\mathrm{x} 10^{4}$ ) and equivalent isotropic displacement parameters $\left(\AA^{2} \mathrm{x}\right.$ $10^{3}$ ) for JF3035FMI. U(eq) is defined as one third of the trace of the orthogonalized $\mathrm{U}^{\mathrm{ij}}$ tensor.

\begin{tabular}{lcccc}
\hline & $\mathrm{x}$ & $\mathrm{y}$ & $\mathrm{z}$ & $\mathrm{U}(\mathrm{eq})$ \\
\hline $\mathrm{S}(1)$ & $6163(1)$ & $5303(1)$ & $7360(1)$ & $20(1)$ \\
$\mathrm{O}(1)$ & $7480(1)$ & $5490(1)$ & $8623(1)$ & $26(1)$ \\
$\mathrm{O}(2)$ & $6211(1)$ & $4806(1)$ & $6793(1)$ & $25(1)$ \\
$\mathrm{N}(1)$ & $5783(1)$ & $5686(1)$ & $5997(1)$ & $20(1)$ \\
$\mathrm{C}(1)$ & $4641(1)$ & $5314(1)$ & $7813(1)$ & $22(1)$ \\
$\mathrm{C}(2)$ & $3299(2)$ & $5059(1)$ & $6904(2)$ & $32(1)$ \\
$\mathrm{C}(3)$ & $2094(2)$ & $5077(1)$ & $7249(2)$ & $39(1)$ \\
$\mathrm{C}(4)$ & $2199(2)$ & $5346(1)$ & $8474(2)$ & $39(1)$ \\
$\mathrm{C}(5)$ & $3559(2)$ & $5589(1)$ & $9380(2)$ & $35(1)$ \\
$\mathrm{C}(6)$ & $4788(2)$ & $5578(1)$ & $9063(2)$ & $26(1)$ \\
$\mathrm{C}(7)$ & $855(2)$ & $5379(1)$ & $8797(2)$ & $57(1)$ \\
$\mathrm{C}(8)$ & $5829(1)$ & $6239(1)$ & $6206(1)$ & $20(1)$ \\
$\mathrm{C}(9)$ & $7340(1)$ & $6453(1)$ & $6362(2)$ & $27(1)$ \\
$\mathrm{C}(10)$ & $7443(4)$ & $6459(1)$ & $4904(4)$ & $29(1)$ \\
$\mathrm{C}(11)$ & $8348(3)$ & $6153(1)$ & $4639(3)$ & $36(1)$ \\
$\mathrm{C}(10 \mathrm{~B})$ & $7706(6)$ & $6282(2)$ & $5144(6)$ & $27(1)$ \\
$\mathrm{C}(11 \mathrm{~B})$ & $7887(4)$ & $6608(1)$ & $4257(4)$ & $32(1)$ \\
$\mathrm{C}(12)$ & $4425(1)$ & $6473(1)$ & $4920(1)$ & $21(1)$ \\
$\mathrm{C}(13)$ & $2984(1)$ & $6396(1)$ & $5091(1)$ & $19(1)$ \\
& & & &
\end{tabular}




\begin{tabular}{lrrrr}
$\mathrm{O}(13)$ & $3182(1)$ & $6708(1)$ & $6319(1)$ & $20(1)$ \\
$\mathrm{C}(14)$ & $1550(1)$ & $6514(1)$ & $3677(1)$ & $20(1)$ \\
$\mathrm{C}(15)$ & $884(1)$ & $6990(1)$ & $3400(1)$ & $24(1)$ \\
$\mathrm{C}(16)$ & $-425(2)$ & $7090(1)$ & $2075(2)$ & $31(1)$ \\
$\mathrm{C}(17)$ & $-1069(2)$ & $6712(1)$ & $1020(2)$ & $34(1)$ \\
$\mathrm{C}(18)$ & $-408(2)$ & $6236(1)$ & $1280(2)$ & $35(1)$ \\
$\mathrm{C}(19)$ & $893(2)$ & $6139(1)$ & $2604(2)$ & $28(1)$ \\
$\mathrm{C}(20)$ & $2132(1)$ & $6611(1)$ & $6894(1)$ & $21(1)$ \\
$\mathrm{C}(21)$ & $2769(1)$ & $6813(1)$ & $8462(1)$ & $20(1)$ \\
$\mathrm{C}(22)$ & $4315(1)$ & $6908(1)$ & $9320(1)$ & $22(1)$ \\
$\mathrm{C}(23)$ & $4883(2)$ & $7075(1)$ & $10784(1)$ & $27(1)$ \\
$\mathrm{C}(24)$ & $3906(2)$ & $7143(1)$ & $11407(2)$ & $30(1)$ \\
$\mathrm{C}(25)$ & $2367(2)$ & $7046(1)$ & $10569(2)$ & $30(1)$ \\
$\mathrm{C}(26)$ & $1797(2)$ & $6885(1)$ & $9099(2)$ & $25(1)$ \\
& & & & \\
\hline
\end{tabular}

Table S10. Bond lengths $[\AA]$ and angles $\left[^{\circ}\right]$ for JF3035FMI.

\begin{tabular}{llll}
\hline $\mathrm{S}(1)-\mathrm{O}(1)$ & $1.4331(9)$ & $\mathrm{C}(7)-\mathrm{H}(7 \mathrm{~B})$ & 0.9800 \\
$\mathrm{~S}(1)-\mathrm{O}(2)$ & $1.4458(9)$ & $\mathrm{C}(7)-\mathrm{H}(7 \mathrm{C})$ & 0.9800 \\
$\mathrm{~S}(1)-\mathrm{N}(1)$ & $1.6112(11)$ & $\mathrm{C}(7)-\mathrm{H}(7 \mathrm{D})$ & 0.9800 \\
$\mathrm{~S}(1)-\mathrm{C}(1)$ & $1.7614(13)$ & $\mathrm{C}(7)-\mathrm{H}(7 \mathrm{E})$ & 0.9800 \\
$\mathrm{~N}(1)-\mathrm{C}(8)$ & $1.4819(15)$ & $\mathrm{C}(7)-\mathrm{H}(7 \mathrm{~F})$ & 0.9800 \\
$\mathrm{~N}(1)-\mathrm{H}(1)$ & $0.869(18)$ & $\mathrm{C}(8)-\mathrm{C}(12)$ & $1.5331(17)$ \\
$\mathrm{C}(1)-\mathrm{C}(6)$ & $1.3918(18)$ & $\mathrm{C}(8)-\mathrm{C}(9)$ & $1.5380(16)$ \\
$\mathrm{C}(1)-\mathrm{C}(2)$ & $\mathrm{C}(8)-\mathrm{H}(8)$ & $0.991(14)$ \\
$\mathrm{C}(2)-\mathrm{C}(3)$ & $1.3959(19)$ & $\mathrm{C}(9)-\mathrm{C}(10 \mathrm{~B})$ & $1.501(5)$ \\
$\mathrm{C}(2)-\mathrm{H}(2)$ & $1.386(2)$ & $\mathrm{C}(9)-\mathrm{C}(10)$ & $1.521(3)$ \\
$\mathrm{C}(3)-\mathrm{C}(4)$ & $0.957(19)$ & $\mathrm{C}(9)-\mathrm{H}(9 \mathrm{~A})$ & 0.9900 \\
$\mathrm{C}(3)-\mathrm{H}(3)$ & $1.390(2)$ & $\mathrm{C}(9)-\mathrm{H}(9 \mathrm{~B})$ & 0.9900 \\
$\mathrm{C}(4)-\mathrm{C}(5)$ & $0.952(19)$ & $\mathrm{C}(9)-\mathrm{H}(9 \mathrm{C})$ & 0.9900 \\
$\mathrm{C}(4)-\mathrm{C}(7)$ & $1.393(2)$ & $\mathrm{C}(9)-\mathrm{H}(9 \mathrm{D})$ & 0.9900 \\
$\mathrm{C}(5)-\mathrm{C}(6)$ & $1.508(2)$ & $\mathrm{C}(10)-\mathrm{C}(11)$ & $1.323(4)$ \\
$\mathrm{C}(5)-\mathrm{H}(5)$ & $1.3908(19)$ & $\mathrm{C}(10)-\mathrm{H}(10)$ & 0.9500 \\
$\mathrm{C}(6)-\mathrm{H}(6)$ & $0.929(18)$ & $\mathrm{C}(11)-\mathrm{H}(11 \mathrm{~A})$ & 0.9500 \\
$\mathrm{C}(7)-\mathrm{H}(7 \mathrm{~A})$ & $0.947(16)$ & $\mathrm{C}(11)-\mathrm{H}(11 \mathrm{~B})$ & 0.9500
\end{tabular}




\begin{tabular}{|c|c|c|c|}
\hline $\mathrm{C}(10 \mathrm{~B})-\mathrm{C}(11 \mathrm{~B})$ & $1.315(5)$ & & \\
\hline $\mathrm{C}(10 \mathrm{~B})-\mathrm{H}(10 \mathrm{~B})$ & 0.9500 & $\mathrm{O}(1)-\mathrm{S}(1)-\mathrm{O}(2)$ & $119.26(5)$ \\
\hline $\mathrm{C}(11 \mathrm{~B})-\mathrm{H}(11 \mathrm{C})$ & 0.9500 & $\mathrm{O}(1)-\mathrm{S}(1)-\mathrm{N}(1)$ & $107.61(6)$ \\
\hline $\mathrm{C}(11 \mathrm{~B})-\mathrm{H}(11 \mathrm{D})$ & 0.9500 & $\mathrm{O}(2)-\mathrm{S}(1)-\mathrm{N}(1)$ & $106.01(5)$ \\
\hline$C(12)-C(13)$ & $1.5243(16)$ & $\mathrm{O}(1)-\mathrm{S}(1)-\mathrm{C}(1)$ & $107.43(6)$ \\
\hline $\mathrm{C}(12)-\mathrm{H}(12 \mathrm{~A})$ & $0.987(15)$ & $\mathrm{O}(2)-\mathrm{S}(1)-\mathrm{C}(1)$ & $107.68(6)$ \\
\hline $\mathrm{C}(12)-\mathrm{H}(12 \mathrm{~B})$ & $0.979(15)$ & $\mathrm{N}(1)-\mathrm{S}(1)-\mathrm{C}(1)$ & $108.49(6)$ \\
\hline $\mathrm{C}(13)-\mathrm{O}(13)$ & $1.4282(14)$ & $\mathrm{C}(8)-\mathrm{N}(1)-\mathrm{S}(1)$ & $121.66(8)$ \\
\hline $\mathrm{C}(13)-\mathrm{C}(14)$ & $1.5157(16)$ & $\mathrm{C}(8)-\mathrm{N}(1)-\mathrm{H}(1)$ & $117.1(11)$ \\
\hline $\mathrm{C}(13)-\mathrm{H}(13)$ & $0.970(14)$ & $\mathrm{S}(1)-\mathrm{N}(1)-\mathrm{H}(1)$ & $114.8(11)$ \\
\hline $\mathrm{O}(13)-\mathrm{C}(20)$ & $1.4248(14)$ & $\mathrm{C}(6)-\mathrm{C}(1)-\mathrm{C}(2)$ & $120.98(12)$ \\
\hline$C(14)-C(15)$ & $1.3927(17)$ & $C(6)-C(1)-S(1)$ & $119.73(10)$ \\
\hline $\mathrm{C}(14)-\mathrm{C}(19)$ & $1.3958(17)$ & $C(2)-C(1)-S(1)$ & $119.29(10)$ \\
\hline$C(15)-C(16)$ & $1.3970(18)$ & $\mathrm{C}(3)-\mathrm{C}(2)-\mathrm{C}(1)$ & $119.06(14)$ \\
\hline $\mathrm{C}(15)-\mathrm{H}(15)$ & $0.981(16)$ & $\mathrm{C}(3)-\mathrm{C}(2)-\mathrm{H}(2)$ & $121.0(11)$ \\
\hline$C(16)-C(17)$ & $1.387(2)$ & $\mathrm{C}(1)-\mathrm{C}(2)-\mathrm{H}(2)$ & 119.9(11) \\
\hline C(16)-H(16) & $0.968(17)$ & $C(2)-C(3)-C(4)$ & $121.18(15)$ \\
\hline C(17)-C(18) & $1.391(2)$ & $\mathrm{C}(2)-\mathrm{C}(3)-\mathrm{H}(3)$ & $118.4(11)$ \\
\hline $\mathrm{C}(17)-\mathrm{H}(17)$ & $0.977(17)$ & $\mathrm{C}(4)-\mathrm{C}(3)-\mathrm{H}(3)$ & $120.4(11)$ \\
\hline C(18)-C(19) & $1.3923(19)$ & $\mathrm{C}(3)-\mathrm{C}(4)-\mathrm{C}(5)$ & $118.70(13)$ \\
\hline C(18)-H(18) & $0.97(2)$ & $\mathrm{C}(3)-\mathrm{C}(4)-\mathrm{C}(7)$ & $120.46(17)$ \\
\hline C(19)-H(19) & $0.972(17)$ & $\mathrm{C}(5)-\mathrm{C}(4)-\mathrm{C}(7)$ & $120.84(16)$ \\
\hline $\mathrm{C}(20)-\mathrm{C}(21)$ & $1.5101(16)$ & $C(6)-C(5)-C(4)$ & $121.44(14)$ \\
\hline $\mathrm{C}(20)-\mathrm{H}(20 \mathrm{~A})$ & $0.981(14)$ & $\mathrm{C}(6)-\mathrm{C}(5)-\mathrm{H}(5)$ & $117.4(11)$ \\
\hline $\mathrm{C}(20)-\mathrm{H}(20 \mathrm{~B})$ & $0.977(15)$ & $\mathrm{C}(4)-\mathrm{C}(5)-\mathrm{H}(5)$ & $121.1(11)$ \\
\hline $\mathrm{C}(21)-\mathrm{C}(26)$ & $1.3915(17)$ & $C(5)-C(6)-C(1)$ & $118.62(14)$ \\
\hline $\mathrm{C}(21)-\mathrm{C}(22)$ & $1.3933(17)$ & $\mathrm{C}(5)-\mathrm{C}(6)-\mathrm{H}(6)$ & $121.9(9)$ \\
\hline$C(22)-C(23)$ & $1.3921(18)$ & $\mathrm{C}(1)-\mathrm{C}(6)-\mathrm{H}(6)$ & 119.4(9) \\
\hline $\mathrm{C}(22)-\mathrm{H}(22)$ & $0.954(15)$ & $\mathrm{C}(4)-\mathrm{C}(7)-\mathrm{H}(7 \mathrm{~A})$ & 109.5 \\
\hline C(23)-C(24) & $1.3837(19)$ & $\mathrm{C}(4)-\mathrm{C}(7)-\mathrm{H}(7 \mathrm{~B})$ & 109.5 \\
\hline $\mathrm{C}(23)-\mathrm{H}(23)$ & $0.962(17)$ & $\mathrm{H}(7 \mathrm{~A})-\mathrm{C}(7)-\mathrm{H}(7 \mathrm{~B})$ & 109.5 \\
\hline$C(24)-C(25)$ & $1.386(2)$ & $\mathrm{C}(4)-\mathrm{C}(7)-\mathrm{H}(7 \mathrm{C})$ & 109.5 \\
\hline C(24)-H(24) & $0.982(17)$ & $\mathrm{H}(7 \mathrm{~A})-\mathrm{C}(7)-\mathrm{H}(7 \mathrm{C})$ & 109.5 \\
\hline$C(25)-C(26)$ & $1.3923(19)$ & $\mathrm{H}(7 \mathrm{~B})-\mathrm{C}(7)-\mathrm{H}(7 \mathrm{C})$ & 109.5 \\
\hline $\mathrm{C}(25)-\mathrm{H}(25)$ & $0.937(18)$ & $\mathrm{H}(7 \mathrm{D})-\mathrm{C}(7)-\mathrm{H}(7 \mathrm{E})$ & 109.5 \\
\hline $\mathrm{C}(26)-\mathrm{H}(26)$ & $0.964(16)$ & $\mathrm{H}(7 \mathrm{D})-\mathrm{C}(7)-\mathrm{H}(7 \mathrm{~F})$ & 109.5 \\
\hline
\end{tabular}




\begin{tabular}{|c|c|c|c|}
\hline $\mathrm{H}(7 \mathrm{E})-\mathrm{C}(7)-\mathrm{H}(7 \mathrm{~F})$ & 109.5 & $\mathrm{H}(12 \mathrm{~A})-\mathrm{C}(12)-\mathrm{H}(12 \mathrm{~B})$ & $109.0(12)$ \\
\hline $\mathrm{N}(1)-\mathrm{C}(8)-\mathrm{C}(12)$ & $108.86(9)$ & $\mathrm{O}(13)-\mathrm{C}(13)-\mathrm{C}(14)$ & $113.29(9)$ \\
\hline $\mathrm{N}(1)-\mathrm{C}(8)-\mathrm{C}(9)$ & $110.24(10)$ & $\mathrm{O}(13)-\mathrm{C}(13)-\mathrm{C}(12)$ & $104.85(9)$ \\
\hline$C(12)-C(8)-C(9)$ & $113.50(10)$ & $\mathrm{C}(14)-\mathrm{C}(13)-\mathrm{C}(12)$ & $112.76(10)$ \\
\hline $\mathrm{N}(1)-\mathrm{C}(8)-\mathrm{H}(8)$ & $108.5(8)$ & $\mathrm{O}(13)-\mathrm{C}(13)-\mathrm{H}(13)$ & $109.0(8)$ \\
\hline $\mathrm{C}(12)-\mathrm{C}(8)-\mathrm{H}(8)$ & $107.2(8)$ & $\mathrm{C}(14)-\mathrm{C}(13)-\mathrm{H}(13)$ & $107.4(8)$ \\
\hline $\mathrm{C}(9)-\mathrm{C}(8)-\mathrm{H}(8)$ & $108.3(8)$ & $\mathrm{C}(12)-\mathrm{C}(13)-\mathrm{H}(13)$ & $109.4(8)$ \\
\hline $\mathrm{C}(10 \mathrm{~B})-\mathrm{C}(9)-\mathrm{C}(8)$ & $113.0(2)$ & $\mathrm{C}(20)-\mathrm{O}(13)-\mathrm{C}(13)$ & $114.30(9)$ \\
\hline $\mathrm{C}(10)-\mathrm{C}(9)-\mathrm{C}(8)$ & $113.33(17)$ & $\mathrm{C}(15)-\mathrm{C}(14)-\mathrm{C}(19)$ & $118.90(11)$ \\
\hline $\mathrm{C}(10)-\mathrm{C}(9)-\mathrm{H}(9 \mathrm{~A})$ & 108.9 & $\mathrm{C}(15)-\mathrm{C}(14)-\mathrm{C}(13)$ & $122.01(11)$ \\
\hline $\mathrm{C}(8)-\mathrm{C}(9)-\mathrm{H}(9 \mathrm{~A})$ & 108.9 & $C(19)-C(14)-C(13)$ & $119.08(11)$ \\
\hline $\mathrm{C}(10)-\mathrm{C}(9)-\mathrm{H}(9 \mathrm{~B})$ & 108.9 & $\mathrm{C}(14)-\mathrm{C}(15)-\mathrm{C}(16)$ & $120.66(12)$ \\
\hline $\mathrm{C}(8)-\mathrm{C}(9)-\mathrm{H}(9 \mathrm{~B})$ & 108.9 & $\mathrm{C}(14)-\mathrm{C}(15)-\mathrm{H}(15)$ & 119.1(9) \\
\hline $\mathrm{H}(9 \mathrm{~A})-\mathrm{C}(9)-\mathrm{H}(9 \mathrm{~B})$ & 107.7 & $\mathrm{C}(16)-\mathrm{C}(15)-\mathrm{H}(15)$ & $120.2(9)$ \\
\hline $\mathrm{C}(10 \mathrm{~B})-\mathrm{C}(9)-\mathrm{H}(9 \mathrm{C})$ & 109.0 & $C(17)-C(16)-C(15)$ & $119.88(13)$ \\
\hline $\mathrm{C}(8)-\mathrm{C}(9)-\mathrm{H}(9 \mathrm{C})$ & 109.0 & $\mathrm{C}(17)-\mathrm{C}(16)-\mathrm{H}(16)$ & $120.0(10)$ \\
\hline $\mathrm{C}(10 \mathrm{~B})-\mathrm{C}(9)-\mathrm{H}(9 \mathrm{D})$ & 109.0 & $\mathrm{C}(15)-\mathrm{C}(16)-\mathrm{H}(16)$ & $120.1(10)$ \\
\hline $\mathrm{C}(8)-\mathrm{C}(9)-\mathrm{H}(9 \mathrm{D})$ & 109.0 & $C(16)-C(17)-C(18)$ & $119.97(13)$ \\
\hline $\mathrm{H}(9 \mathrm{C})-\mathrm{C}(9)-\mathrm{H}(9 \mathrm{D})$ & 107.8 & $\mathrm{C}(16)-\mathrm{C}(17)-\mathrm{H}(17)$ & $120.0(10)$ \\
\hline $\mathrm{C}(11)-\mathrm{C}(10)-\mathrm{C}(9)$ & $122.6(3)$ & $\mathrm{C}(18)-\mathrm{C}(17)-\mathrm{H}(17)$ & $120.1(10)$ \\
\hline $\mathrm{C}(11)-\mathrm{C}(10)-\mathrm{H}(10)$ & 118.7 & $\mathrm{C}(17)-\mathrm{C}(18)-\mathrm{C}(19)$ & $119.97(13)$ \\
\hline $\mathrm{C}(9)-\mathrm{C}(10)-\mathrm{H}(10)$ & 118.7 & $\mathrm{C}(17)-\mathrm{C}(18)-\mathrm{H}(18)$ & 121.1(11) \\
\hline $\mathrm{C}(10)-\mathrm{C}(11)-\mathrm{H}(11 \mathrm{~A})$ & 120.0 & $\mathrm{C}(19)-\mathrm{C}(18)-\mathrm{H}(18)$ & $118.9(11)$ \\
\hline $\mathrm{C}(10)-\mathrm{C}(11)-\mathrm{H}(11 \mathrm{~B})$ & 120.0 & $\mathrm{C}(18)-\mathrm{C}(19)-\mathrm{C}(14)$ & $120.62(13)$ \\
\hline $\mathrm{H}(11 \mathrm{~A})-\mathrm{C}(11)-\mathrm{H}(11 \mathrm{~B})$ & 120.0 & $\mathrm{C}(18)-\mathrm{C}(19)-\mathrm{H}(19)$ & 120.1(10) \\
\hline$C(11 B)-C(10 B)-C(9)$ & 121.1(4) & $\mathrm{C}(14)-\mathrm{C}(19)-\mathrm{H}(19)$ & $119.3(10)$ \\
\hline $\mathrm{C}(11 \mathrm{~B})-\mathrm{C}(10 \mathrm{~B})-\mathrm{H}(10 \mathrm{~B})$ & 119.4 & $\mathrm{O}(13)-\mathrm{C}(20)-\mathrm{C}(21)$ & 109.28(9) \\
\hline $\mathrm{C}(9)-\mathrm{C}(10 \mathrm{~B})-\mathrm{H}(10 \mathrm{~B})$ & 119.4 & $\mathrm{O}(13)-\mathrm{C}(20)-\mathrm{H}(20 \mathrm{~A})$ & $109.0(8)$ \\
\hline $\mathrm{C}(10 \mathrm{~B})-\mathrm{C}(11 \mathrm{~B})-\mathrm{H}(11 \mathrm{C})$ & 120.0 & $\mathrm{C}(21)-\mathrm{C}(20)-\mathrm{H}(20 \mathrm{~A})$ & $110.6(8)$ \\
\hline $\mathrm{C}(10 \mathrm{~B})-\mathrm{C}(11 \mathrm{~B})-\mathrm{H}(11 \mathrm{D})$ & 120.0 & $\mathrm{O}(13)-\mathrm{C}(20)-\mathrm{H}(20 \mathrm{~B})$ & $110.7(9)$ \\
\hline $\mathrm{H}(11 \mathrm{C})-\mathrm{C}(11 \mathrm{~B})-\mathrm{H}(11 \mathrm{D})$ & 120.0 & $\mathrm{C}(21)-\mathrm{C}(20)-\mathrm{H}(20 \mathrm{~B})$ & $111.8(9)$ \\
\hline $\mathrm{C}(13)-\mathrm{C}(12)-\mathrm{C}(8)$ & $112.44(10)$ & $\mathrm{H}(20 \mathrm{~A})-\mathrm{C}(20)-\mathrm{H}(20 \mathrm{~B})$ & $105.4(12)$ \\
\hline $\mathrm{C}(13)-\mathrm{C}(12)-\mathrm{H}(12 \mathrm{~A})$ & $107.4(8)$ & $\mathrm{C}(26)-\mathrm{C}(21)-\mathrm{C}(22)$ & $118.78(11)$ \\
\hline $\mathrm{C}(8)-\mathrm{C}(12)-\mathrm{H}(12 \mathrm{~A})$ & $108.5(8)$ & $\mathrm{C}(26)-\mathrm{C}(21)-\mathrm{C}(20)$ & $119.19(11)$ \\
\hline $\mathrm{C}(13)-\mathrm{C}(12)-\mathrm{H}(12 \mathrm{~B})$ & 109.8(9) & $\mathrm{C}(22)-\mathrm{C}(21)-\mathrm{C}(20)$ & $121.96(11)$ \\
\hline $\mathrm{C}(8)-\mathrm{C}(12)-\mathrm{H}(12 \mathrm{~B})$ & $109.6(9)$ & $C(23)-C(22)-C(21)$ & $120.91(12)$ \\
\hline
\end{tabular}




$\begin{array}{llll}\mathrm{C}(23)-\mathrm{C}(22)-\mathrm{H}(22) & 120.4(9) & \mathrm{C}(25)-\mathrm{C}(24)-\mathrm{H}(24) & 120.8(10) \\ \mathrm{C}(21)-\mathrm{C}(22)-\mathrm{H}(22) & 118.7(9) & \mathrm{C}(24)-\mathrm{C}(25)-\mathrm{C}(26) & 120.33(12) \\ \mathrm{C}(24)-\mathrm{C}(23)-\mathrm{C}(22) & 119.78(12) & \mathrm{C}(24)-\mathrm{C}(25)-\mathrm{H}(25) & 121.5(11) \\ \mathrm{C}(24)-\mathrm{C}(23)-\mathrm{H}(23) & 120.6(10) & \mathrm{C}(26)-\mathrm{C}(25)-\mathrm{H}(25) & 118.2(11) \\ \mathrm{C}(22)-\mathrm{C}(23)-\mathrm{H}(23) & 119.6(10) & \mathrm{C}(21)-\mathrm{C}(26)-\mathrm{C}(25) & 120.33(12) \\ \mathrm{C}(23)-\mathrm{C}(24)-\mathrm{C}(25) & 119.87(12) & \mathrm{C}(21)-\mathrm{C}(26)-\mathrm{H}(26) & 119.1(9) \\ \mathrm{C}(23)-\mathrm{C}(24)-\mathrm{H}(24) & 119.3(10) & \mathrm{C}(25)-\mathrm{C}(26)-\mathrm{H}(26) & 120.5(9)\end{array}$

Symmetry transformations used to generate equivalent atoms:

Table S11. Anisotropic displacement parameters $\left(\AA^{2} \times 10^{3}\right)$ for JF3035FMI. The anisotropic displacement factor exponent takes the form: $-2 p^{2}\left[h^{2} a^{* 2} U^{11}+\ldots+2 h k a^{*} b^{*} U^{12}\right]$

\begin{tabular}{|c|c|c|c|c|c|c|}
\hline & $\mathrm{U}^{11}$ & $\mathrm{U}^{22}$ & $\mathrm{U}^{33}$ & $\mathrm{U}^{23}$ & $\mathrm{U}^{13}$ & $\mathrm{U}^{12}$ \\
\hline$S(1)$ & $17(1)$ & $22(1)$ & 19(1) & $-4(1)$ & $7(1)$ & $0(1)$ \\
\hline $\mathrm{O}(1)$ & $22(1)$ & $30(1)$ & 21(1) & $-4(1)$ & $5(1)$ & $-3(1)$ \\
\hline $\mathrm{O}(2)$ & $23(1)$ & $24(1)$ & $24(1)$ & $-5(1)$ & $7(1)$ & $4(1)$ \\
\hline $\mathrm{N}(1)$ & $20(1)$ & $23(1)$ & 18(1) & $-5(1)$ & $9(1)$ & $-2(1)$ \\
\hline $\mathrm{C}(1)$ & $24(1)$ & $20(1)$ & $28(1)$ & $3(1)$ & $15(1)$ & $2(1)$ \\
\hline$C(2)$ & $26(1)$ & $25(1)$ & $44(1)$ & $-3(1)$ & $17(1)$ & $-1(1)$ \\
\hline C(3) & $29(1)$ & $29(1)$ & $66(1)$ & $6(1)$ & $26(1)$ & $0(1)$ \\
\hline$C(4)$ & $43(1)$ & $29(1)$ & $63(1)$ & $24(1)$ & $39(1)$ & $16(1)$ \\
\hline$C(5)$ & $52(1)$ & $29(1)$ & $40(1)$ & $15(1)$ & $34(1)$ & $17(1)$ \\
\hline$C(6)$ & $36(1)$ & $21(1)$ & $27(1)$ & $6(1)$ & $18(1)$ & $7(1)$ \\
\hline$C(7)$ & $56(1)$ & $56(1)$ & $88(1)$ & $40(1)$ & $58(1)$ & $28(1)$ \\
\hline$C(8)$ & 19(1) & $22(1)$ & 19(1) & $-4(1)$ & $9(1)$ & $-1(1)$ \\
\hline $\mathrm{C}(9)$ & 19(1) & $30(1)$ & $29(1)$ & $-4(1)$ & $9(1)$ & $-5(1)$ \\
\hline$C(10)$ & $22(2)$ & $30(2)$ & $36(2)$ & $6(1)$ & $15(1)$ & $-1(1)$ \\
\hline $\mathrm{C}(11)$ & $28(1)$ & $42(1)$ & $42(1)$ & $-2(1)$ & 21(1) & $-7(1)$ \\
\hline $\mathrm{C}(10 \mathrm{~B})$ & $22(2)$ & $23(2)$ & $39(2)$ & $1(2)$ & $16(2)$ & $1(2)$ \\
\hline$C(11 B)$ & $27(2)$ & $41(2)$ & $33(2)$ & $0(2)$ & $18(2)$ & $-3(1)$ \\
\hline$C(12)$ & $20(1)$ & $24(1)$ & 19(1) & $-2(1)$ & $9(1)$ & $-1(1)$ \\
\hline $\mathrm{C}(13)$ & 21(1) & 19(1) & 19(1) & $-2(1)$ & $9(1)$ & $0(1)$ \\
\hline $\mathrm{O}(13)$ & $20(1)$ & $24(1)$ & $18(1)$ & $-4(1)$ & $10(1)$ & $-4(1)$ \\
\hline$C(14)$ & $18(1)$ & $24(1)$ & 20(1) & $-3(1)$ & $10(1)$ & $-1(1)$ \\
\hline
\end{tabular}




\begin{tabular}{lllllll}
$\mathrm{C}(15)$ & $26(1)$ & $26(1)$ & $20(1)$ & $-3(1)$ & $11(1)$ & $3(1)$ \\
$\mathrm{C}(16)$ & $29(1)$ & $39(1)$ & $24(1)$ & $1(1)$ & $12(1)$ & $13(1)$ \\
$\mathrm{C}(17)$ & $20(1)$ & $57(1)$ & $22(1)$ & $-6(1)$ & $6(1)$ & $7(1)$ \\
$\mathrm{C}(18)$ & $24(1)$ & $46(1)$ & $28(1)$ & $-17(1)$ & $7(1)$ & $-4(1)$ \\
$\mathrm{C}(19)$ & $24(1)$ & $28(1)$ & $29(1)$ & $-10(1)$ & $11(1)$ & $-1(1)$ \\
$\mathrm{C}(20)$ & $19(1)$ & $24(1)$ & $20(1)$ & $0(1)$ & $10(1)$ & $-1(1)$ \\
$\mathrm{C}(21)$ & $24(1)$ & $17(1)$ & $19(1)$ & $2(1)$ & $11(1)$ & $1(1)$ \\
$\mathrm{C}(22)$ & $24(1)$ & $25(1)$ & $21(1)$ & $2(1)$ & $12(1)$ & $0(1)$ \\
$\mathrm{C}(23)$ & $28(1)$ & $29(1)$ & $21(1)$ & $1(1)$ & $8(1)$ & $-2(1)$ \\
$\mathrm{C}(24)$ & $42(1)$ & $30(1)$ & $19(1)$ & $-1(1)$ & $15(1)$ & $-3(1)$ \\
$\mathrm{C}(25)$ & $38(1)$ & $34(1)$ & $29(1)$ & $-2(1)$ & $24(1)$ & $-1(1)$ \\
$\mathrm{C}(26)$ & $25(1)$ & $27(1)$ & $27(1)$ & $0(1)$ & $15(1)$ & $-1(1)$ \\
& & & & & & \\
\hline
\end{tabular}

Table S12. Hydrogen coordinates ( x 10 $0^{4}$ ) and isotropic displacement parameters $\left(\AA^{2} \times 10^{3}\right)$ for JF3035FMI.

\begin{tabular}{lcccc}
\hline & x & y & z & U(eq) \\
& & & & \\
& & & & \\
$H(1)$ & $5150(20)$ & $5570(6)$ & $5130(20)$ & $37(4)$ \\
$\mathrm{H}(2)$ & $3210(20)$ & $4883(7)$ & $6040(20)$ & $46(5)$ \\
$\mathrm{H}(3)$ & $1180(20)$ & $4904(7)$ & $6620(20)$ & $48(5)$ \\
$\mathrm{H}(5)$ & $3666(19)$ & $5776(6)$ & $10200(20)$ & $40(5)$ \\
$\mathrm{H}(6)$ & $5728(18)$ & $5733(6)$ & $9693(17)$ & $28(4)$ \\
$\mathrm{H}(7 \mathrm{~A})$ & 1128 & 5582 & 9695 & 85 \\
$\mathrm{H}(7 \mathrm{~B})$ & -2 & 5537 & 7952 & 85 \\
$\mathrm{H}(7 \mathrm{C})$ & 564 & 5040 & 8954 & 85 \\
$\mathrm{H}(7 \mathrm{D})$ & -1 & 5190 & 8039 & 85 \\
$\mathrm{H}(7 \mathrm{E})$ & 1129 & 5235 & 9782 & 85 \\
$\mathrm{H}(7 \mathrm{~F})$ & 563 & 5732 & 8780 & 85 \\
$\mathrm{H}(8)$ & $5760(15)$ & $6312(5)$ & $7137(15)$ & $17(3)$ \\
$\mathrm{H}(9 \mathrm{~A})$ & 7464 & 6801 & 6752 & 32 \\
$\mathrm{H}(9 \mathrm{~B})$ & 8188 & 6249 & 7098 & 32 \\
$\mathrm{H}(9 \mathrm{C})$ & 7289 & 6826 & 6353 & 32 \\
$\mathrm{H}(9 \mathrm{D})$ & 8172 & 6348 & 7335 & 32 \\
& & & &
\end{tabular}




\begin{tabular}{lllll}
$\mathrm{H}(10)$ & 6834 & 6692 & 4153 & 34 \\
$\mathrm{H}(11 \mathrm{~A})$ & 8966 & 5917 & 5375 & 43 \\
$\mathrm{H}(11 \mathrm{~B})$ & 8378 & 6169 & 3713 & 43 \\
$\mathrm{H}(10 \mathrm{~B})$ & 7807 & 5932 & 5012 & 32 \\
$\mathrm{H}(11 \mathrm{C})$ & 7787 & 6958 & 4382 & 39 \\
$\mathrm{H}(11 \mathrm{D})$ & 8118 & 6493 & 3492 & 39 \\
$\mathrm{H}(12 \mathrm{~A})$ & $4589(16)$ & $6839(6)$ & $4910(16)$ & $21(3)$ \\
$\mathrm{H}(12 \mathrm{~B})$ & $4289(16)$ & $6328(6)$ & $3974(17)$ & $24(4)$ \\
$\mathrm{H}(13)$ & $2920(15)$ & $6048(5)$ & $5342(15)$ & $17(3)$ \\
$\mathrm{H}(15)$ & $1340(17)$ & $7255(6)$ & $4153(17)$ & $28(4)$ \\
$\mathrm{H}(16)$ & $-889(19)$ & $7420(7)$ & $1898(19)$ & $38(4)$ \\
$\mathrm{H}(17)$ & $-1985(19)$ & $6781(6)$ & $93(19)$ & $39(4)$ \\
$\mathrm{H}(18)$ & $-840(20)$ & $5970(7)$ & $550(20)$ & $52(5)$ \\
$\mathrm{H}(19)$ & $1359(19)$ & $5807(6)$ & $2785(18)$ & $35(4)$ \\
$\mathrm{H}(20 \mathrm{~A})$ & $1960(15)$ & $6247(5)$ & $6879(15)$ & $20(3)$ \\
$\mathrm{H}(20 \mathrm{~B})$ & $1139(17)$ & $6756(6)$ & $6247(16)$ & $25(4)$ \\
$\mathrm{H}(22)$ & $4981(17)$ & $6857(6)$ & $8883(17)$ & $27(4)$ \\
$\mathrm{H}(23)$ & $5954(19)$ & $7142(6)$ & $11356(18)$ & $33(4)$ \\
$\mathrm{H}(24)$ & $4319(19)$ & $7252(6)$ & $12450(19)$ & $38(4)$ \\
$\mathrm{H}(25)$ & $1680(20)$ & $7086(6)$ & $10969(19)$ & $42(5)$ \\
$\mathrm{H}(26)$ & $725(18)$ & $6822(6)$ & $8509(17)$ & $29(4)$ \\
& & & & \\
\hline & & & &
\end{tabular}


Table S13. Torsion angles $\left[^{\circ}\right]$ for JF3035FMI.

\begin{tabular}{|c|c|c|c|}
\hline $\mathrm{O}(1)-\mathrm{S}(1)-\mathrm{N}(1)-\mathrm{C}(8)$ & $-43.67(10)$ & $\mathrm{C}(9)-\mathrm{C}(8)-\mathrm{C}(12)-\mathrm{C}(13)$ & $-160.61(10)$ \\
\hline $\mathrm{O}(2)-\mathrm{S}(1)-\mathrm{N}(1)-\mathrm{C}(8)$ & $-172.32(9)$ & $\mathrm{C}(8)-\mathrm{C}(12)-\mathrm{C}(13)-\mathrm{O}(13)$ & $69.67(12)$ \\
\hline $\mathrm{C}(1)-\mathrm{S}(1)-\mathrm{N}(1)-\mathrm{C}(8)$ & $72.27(10)$ & $\mathrm{C}(8)-\mathrm{C}(12)-\mathrm{C}(13)-\mathrm{C}(14)$ & $-166.64(10)$ \\
\hline $\mathrm{O}(1)-\mathrm{S}(1)-\mathrm{C}(1)-\mathrm{C}(6)$ & $13.08(12)$ & $\mathrm{C}(14)-\mathrm{C}(13)-\mathrm{O}(13)-\mathrm{C}(20)$ & $70.15(12)$ \\
\hline $\mathrm{O}(2)-\mathrm{S}(1)-\mathrm{C}(1)-\mathrm{C}(6)$ & $142.70(10)$ & $\mathrm{C}(12)-\mathrm{C}(13)-\mathrm{O}(13)-\mathrm{C}(20)$ & $-166.50(9)$ \\
\hline $\mathrm{N}(1)-\mathrm{S}(1)-\mathrm{C}(1)-\mathrm{C}(6)$ & $-102.98(11)$ & $\mathrm{O}(13)-\mathrm{C}(13)-\mathrm{C}(14)-\mathrm{C}(15)$ & $26.51(16)$ \\
\hline $\mathrm{O}(1)-\mathrm{S}(1)-\mathrm{C}(1)-\mathrm{C}(2)$ & $-167.28(10)$ & $\mathrm{C}(12)-\mathrm{C}(13)-\mathrm{C}(14)-\mathrm{C}(15)$ & $-92.38(13)$ \\
\hline $\mathrm{O}(2)-\mathrm{S}(1)-\mathrm{C}(1)-\mathrm{C}(2)$ & $-37.65(12)$ & $\mathrm{O}(13)-\mathrm{C}(13)-\mathrm{C}(14)-\mathrm{C}(19)$ & $-154.90(11)$ \\
\hline $\mathrm{N}(1)-\mathrm{S}(1)-\mathrm{C}(1)-\mathrm{C}(2)$ & $76.66(11)$ & $\mathrm{C}(12)-\mathrm{C}(13)-\mathrm{C}(14)-\mathrm{C}(19)$ & $86.21(14)$ \\
\hline$C(6)-C(1)-C(2)-C(3)$ & $0.9(2)$ & $\mathrm{C}(19)-\mathrm{C}(14)-\mathrm{C}(15)-\mathrm{C}(16)$ & $0.35(19)$ \\
\hline$S(1)-C(1)-C(2)-C(3)$ & $-178.74(11)$ & $C(13)-C(14)-C(15)-C(16)$ & $178.95(12)$ \\
\hline $\mathrm{C}(1)-\mathrm{C}(2)-\mathrm{C}(3)-\mathrm{C}(4)$ & $0.4(2)$ & $\mathrm{C}(14)-\mathrm{C}(15)-\mathrm{C}(16)-\mathrm{C}(17)$ & $-0.1(2)$ \\
\hline$C(2)-C(3)-C(4)-C(5)$ & $-1.6(2)$ & $\mathrm{C}(15)-\mathrm{C}(16)-\mathrm{C}(17)-\mathrm{C}(18)$ & $-0.3(2)$ \\
\hline$C(2)-C(3)-C(4)-C(7)$ & $177.51(14)$ & $\mathrm{C}(16)-\mathrm{C}(17)-\mathrm{C}(18)-\mathrm{C}(19)$ & $0.5(2)$ \\
\hline $\mathrm{C}(3)-\mathrm{C}(4)-\mathrm{C}(5)-\mathrm{C}(6)$ & $1.6(2)$ & $\mathrm{C}(17)-\mathrm{C}(18)-\mathrm{C}(19)-\mathrm{C}(14)$ & $-0.2(2)$ \\
\hline$C(7)-C(4)-C(5)-C(6)$ & $-177.52(13)$ & $C(15)-C(14)-C(19)-C(18)$ & $-0.2(2)$ \\
\hline $\mathrm{C}(4)-\mathrm{C}(5)-\mathrm{C}(6)-\mathrm{C}(1)$ & $-0.37(19)$ & $\mathrm{C}(13)-\mathrm{C}(14)-\mathrm{C}(19)-\mathrm{C}(18)$ & $-178.79(12)$ \\
\hline$C(2)-C(1)-C(6)-C(5)$ & $-0.91(19)$ & $\mathrm{C}(13)-\mathrm{O}(13)-\mathrm{C}(20)-\mathrm{C}(21)$ & $159.63(9)$ \\
\hline $\mathrm{S}(1)-\mathrm{C}(1)-\mathrm{C}(6)-\mathrm{C}(5)$ & $178.73(9)$ & $\mathrm{O}(13)-\mathrm{C}(20)-\mathrm{C}(21)-\mathrm{C}(26)$ & $162.72(10)$ \\
\hline$S(1)-N(1)-C(8)-C(12)$ & $-135.71(9)$ & $\mathrm{O}(13)-\mathrm{C}(20)-\mathrm{C}(21)-\mathrm{C}(22)$ & $-20.30(15)$ \\
\hline $\mathrm{S}(1)-\mathrm{N}(1)-\mathrm{C}(8)-\mathrm{C}(9)$ & $99.19(11)$ & $\mathrm{C}(26)-\mathrm{C}(21)-\mathrm{C}(22)-\mathrm{C}(23)$ & $-0.40(18)$ \\
\hline $\mathrm{N}(1)-\mathrm{C}(8)-\mathrm{C}(9)-\mathrm{C}(10 \mathrm{~B})$ & $50.6(2)$ & $C(20)-C(21)-C(22)-C(23)$ & $-177.40(11)$ \\
\hline $\mathrm{C}(12)-\mathrm{C}(8)-\mathrm{C}(9)-\mathrm{C}(10 \mathrm{~B})$ & $-71.8(2)$ & $C(21)-C(22)-C(23)-C(24)$ & $0.75(19)$ \\
\hline $\mathrm{N}(1)-\mathrm{C}(8)-\mathrm{C}(9)-\mathrm{C}(10)$ & $72.88(18)$ & $C(22)-C(23)-C(24)-C(25)$ & $-0.3(2)$ \\
\hline $\mathrm{C}(12)-\mathrm{C}(8)-\mathrm{C}(9)-\mathrm{C}(10)$ & $-49.53(19)$ & $C(23)-C(24)-C(25)-C(26)$ & $-0.6(2)$ \\
\hline $\mathrm{C}(8)-\mathrm{C}(9)-\mathrm{C}(10)-\mathrm{C}(11)$ & $-109.1(3)$ & $C(22)-C(21)-C(26)-C(25)$ & $-0.43(18)$ \\
\hline $\mathrm{C}(8)-\mathrm{C}(9)-\mathrm{C}(10 \mathrm{~B})-\mathrm{C}(11 \mathrm{~B})$ & $120.5(4)$ & $C(20)-C(21)-C(26)-C(25)$ & $176.65(12)$ \\
\hline $\mathrm{N}(1)-\mathrm{C}(8)-\mathrm{C}(12)-\mathrm{C}(13)$ & $76.22(12)$ & $C(24)-C(25)-C(26)-C(21)$ & $0.9(2)$ \\
\hline
\end{tabular}

Symmetry transformations used to generate equivalent atoms: 
Table S14. Hydrogen bonds for JF3035FMI [ $\AA$ and ${ }^{\circ}$ ].

\begin{tabular}{lllll}
\hline $\mathrm{D}-\mathrm{H} . . . \mathrm{A}$ & $\mathrm{d}(\mathrm{D}-\mathrm{H})$ & $\mathrm{d}(\mathrm{H} \ldots \mathrm{A})$ & $\mathrm{d}(\mathrm{D} \ldots \mathrm{A})$ & $<(\mathrm{DHA})$ \\
\hline $\mathrm{N}(1)-\mathrm{H}(1) \ldots \mathrm{O}(2) \# 1$ & $0.869(18)$ & $2.045(18)$ & $2.9077(14)$ & $171.4(16)$ \\
\hline
\end{tabular}

Symmetry transformations used to generate equivalent atoms:

$\# 1-\mathrm{x}+1,-\mathrm{y}+1,-\mathrm{z}+1$

X-ray crystallographic data for compound S7

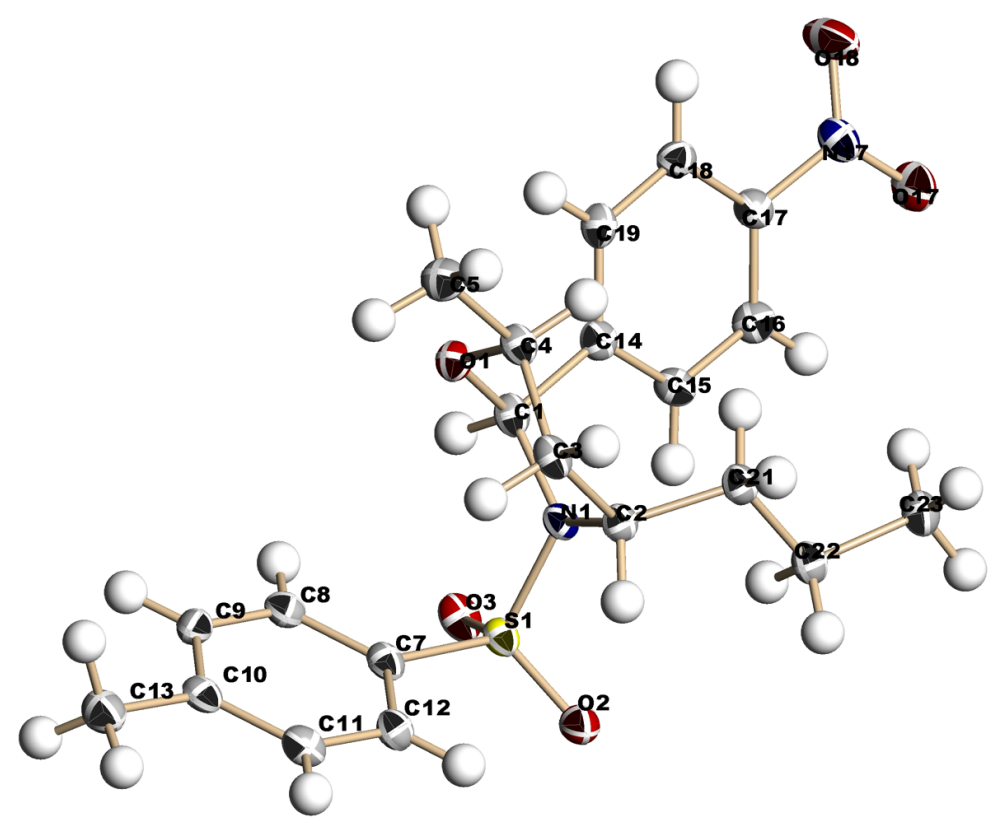

Table S15. Crystal data and structure refinement for $\left[\mathrm{C}_{21} \mathrm{H}_{26} \mathrm{~N}_{2} \mathrm{O} \mathrm{O}_{5} \mathrm{~S}\right]$.

Identification code

Empirical formula

Formula weight

Temperature

Wavelength

Crystal system

Space group

Unit cell dimensions
JF2976KTW42 (AL-III-189-A) (2-Comp Twin)

C21 H26 N2 O5 S

418.50

100(2) K

$0.71073 \AA$

Orthorhombic

Pbca

$\mathrm{a}=7.7823(8) \AA \quad \alpha=90^{\circ}$. 
Volume

Z

Density (calculated)

Absorption coefficient

$\mathrm{F}(000)$

Crystal size

Crystal color and habit

Diffractometer

Theta range for data collection

Index ranges

Reflections collected

Independent reflections

Observed reflections (I > 2sigma(I))

Completeness to theta $=25.242^{\circ}$

Absorption correction

Max. and min. transmission

Solution method

Refinement method

Data / restraints / parameters

Goodness-of-fit on $\mathrm{F}^{2}$

Final $R$ indices [I $>2 \operatorname{sigma}(\mathrm{I})]$

$\mathrm{R}$ indices (all data)

Extinction coefficient

Largest diff. peak and hole $\begin{array}{ll}\mathrm{b}=16.8403(17) \AA & \beta=90^{\circ} . \\ \mathrm{c}=31.222(3) \AA & \gamma=90^{\circ} .\end{array}$

4091.9(7) $\AA^{3}$

8

$1.359 \mathrm{Mg} / \mathrm{m}^{3}$

$0.194 \mathrm{~mm}^{-1}$

1776

$0.439 \times 0.396 \times 0.060 \mathrm{~mm}^{3}$

Colorless Plate

Bruker Photon100 CMOS

2.419 to $25.242^{\circ}$.

$-9<=\mathrm{h}<=9,-19<=\mathrm{k}<=19,-37<=\mathrm{l}<=37$

6581

$3582[\mathrm{R}(\mathrm{int})=0.0317]$

2908

$96.4 \%$

Semi-empirical from equivalents

0.9575 and 0.5829

SHELXT (Sheldrick, 2014)

SHELXL-2017/1 (Sheldrick, 2017) Full-matrix least-squares on F ${ }^{2}$ $3582 / 0 / 266$

1.092

$\mathrm{R} 1=0.0563, \mathrm{wR} 2=0.1307$

$\mathrm{R} 1=0.0753, \mathrm{wR} 2=0.1387$

$0.0042(4)$

0.328 and -0.345 e. $\AA^{-3}$ 
Table S16. Atomic coordinates ( x 10 $)$ and equivalent isotropic displacement parameters $\left(\AA^{2} \mathrm{x}\right.$ $10^{3}$ ) for JF2976KTW42. U(eq) is defined as one third of the trace of the orthogonalized $\mathrm{U}^{\mathrm{ij}}$ tensor.

\begin{tabular}{|c|c|c|c|c|}
\hline & $\mathrm{x}$ & $\mathrm{y}$ & $\mathrm{z}$ & $\mathrm{U}(\mathrm{eq})$ \\
\hline $\mathrm{S}(1)$ & $5405(1)$ & 6839(1) & 5997(1) & $21(1)$ \\
\hline $\mathrm{C}(1)$ & $6587(4)$ & $5411(2)$ & $6238(1)$ & $23(1)$ \\
\hline $\mathrm{N}(1)$ & 6911(3) & $6274(1)$ & 6203(1) & $20(1)$ \\
\hline $\mathrm{O}(1)$ & $7487(2)$ & $4968(1)$ & 5929(1) & $23(1)$ \\
\hline$C(2)$ & $8735(3)$ & 6533(2) & 6143(1) & $21(1)$ \\
\hline $\mathrm{O}(2)$ & $5830(3)$ & $7642(1)$ & $6105(1)$ & $27(1)$ \\
\hline$C(3)$ & $9622(4)$ & 5984(2) & 5819(1) & $24(1)$ \\
\hline $\mathrm{O}(3)$ & $3795(2)$ & $6507(1)$ & 6131(1) & $28(1)$ \\
\hline C(4) & 9332(4) & $5120(2)$ & 5921(1) & $23(1)$ \\
\hline$C(5)$ & $10054(4)$ & $4551(2)$ & $5589(1)$ & $30(1)$ \\
\hline$C(7)$ & $5517(4)$ & 6754(2) & 5434(1) & $22(1)$ \\
\hline$C(8)$ & 4693(4) & 6121(2) & $5232(1)$ & $23(1)$ \\
\hline$C(9)$ & 4791(4) & 6043(2) & 4793(1) & $24(1)$ \\
\hline$C(10)$ & $5729(4)$ & $6586(2)$ & $4545(1)$ & $23(1)$ \\
\hline $\mathrm{C}(11)$ & $6530(4)$ & $7221(2)$ & 4754(1) & $25(1)$ \\
\hline$C(12)$ & $6435(4)$ & $7305(2)$ & 5193(1) & $24(1)$ \\
\hline$C(13)$ & $5844(4)$ & 6491(2) & $4065(1)$ & $31(1)$ \\
\hline$C(14)$ & 6901(4) & $5112(2)$ & 6694(1) & 21(1) \\
\hline$C(15)$ & $6328(4)$ & $5566(2)$ & $7039(1)$ & $23(1)$ \\
\hline$C(16)$ & $6563(4)$ & $5310(2)$ & $7456(1)$ & $25(1)$ \\
\hline$C(17)$ & 7361(4) & $4586(2)$ & 7522(1) & $23(1)$ \\
\hline $\mathrm{N}(17)$ & 7639(3) & $4320(2)$ & 7966(1) & $27(1)$ \\
\hline $\mathrm{O}(17)$ & $7345(3)$ & $4788(1)$ & $8258(1)$ & $35(1)$ \\
\hline$C(18)$ & 7889(4) & $4104(2)$ & $7187(1)$ & $23(1)$ \\
\hline $\mathrm{O}(18)$ & $8166(4)$ & $3643(2)$ & $8023(1)$ & $47(1)$ \\
\hline C(19) & 7649(4) & $4374(2)$ & 6772(1) & $24(1)$ \\
\hline $\mathrm{C}(21)$ & $9638(4)$ & $6594(2)$ & $6577(1)$ & $22(1)$ \\
\hline$C(22)$ & $8886(4)$ & $7241(2)$ & $6865(1)$ & $25(1)$ \\
\hline$C(23)$ & $9534(4)$ & 7191(2) & $7326(1)$ & $27(1)$ \\
\hline
\end{tabular}


Table S17. Bond lengths $[\AA]$ and angles $\left[^{\circ}\right]$ for JF2976KTW42.

\begin{tabular}{|c|c|c|c|}
\hline $\mathrm{S}(1)-\mathrm{O}(2)$ & $1.434(2)$ & $\mathrm{C}(13)-\mathrm{H}(13 \mathrm{C})$ & 0.9800 \\
\hline $\mathrm{S}(1)-\mathrm{O}(3)$ & $1.435(2)$ & $\mathrm{C}(14)-\mathrm{C}(19)$ & $1.394(4)$ \\
\hline $\mathrm{S}(1)-\mathrm{N}(1)$ & $1.641(2)$ & $\mathrm{C}(14)-\mathrm{C}(15)$ & $1.395(4)$ \\
\hline$S(1)-C(7)$ & $1.765(3)$ & $C(15)-C(16)$ & $1.382(4)$ \\
\hline $\mathrm{C}(1)-\mathrm{O}(1)$ & $1.406(3)$ & $\mathrm{C}(15)-\mathrm{H}(15)$ & 0.9500 \\
\hline $\mathrm{C}(1)-\mathrm{N}(1)$ & $1.479(4)$ & $C(16)-C(17)$ & $1.384(4)$ \\
\hline$C(1)-C(14)$ & $1.531(4)$ & $\mathrm{C}(16)-\mathrm{H}(16)$ & 0.9500 \\
\hline $\mathrm{C}(1)-\mathrm{H}(1)$ & 1.0000 & $C(17)-C(18)$ & $1.388(4)$ \\
\hline $\mathrm{N}(1)-\mathrm{C}(2)$ & $1.497(3)$ & $\mathrm{C}(17)-\mathrm{N}(17)$ & $1.470(3)$ \\
\hline $\mathrm{O}(1)-\mathrm{C}(4)$ & $1.459(3)$ & $\mathrm{N}(17)-\mathrm{O}(18)$ & $1.225(3)$ \\
\hline$C(2)-C(21)$ & $1.530(4)$ & $\mathrm{N}(17)-\mathrm{O}(17)$ & $1.226(3)$ \\
\hline $\mathrm{C}(2)-\mathrm{C}(3)$ & $1.534(4)$ & $C(18)-C(19)$ & $1.385(4)$ \\
\hline $\mathrm{C}(2)-\mathrm{H}(2)$ & 1.0000 & $\mathrm{C}(18)-\mathrm{H}(18)$ & 0.9500 \\
\hline $\mathrm{C}(3)-\mathrm{C}(4)$ & $1.507(4)$ & $\mathrm{C}(19)-\mathrm{H}(19)$ & 0.9500 \\
\hline $\mathrm{C}(3)-\mathrm{H}(3 \mathrm{~A})$ & 0.9900 & $C(21)-C(22)$ & $1.530(4)$ \\
\hline $\mathrm{C}(3)-\mathrm{H}(3 \mathrm{~B})$ & 0.9900 & $\mathrm{C}(21)-\mathrm{H}(21 \mathrm{~A})$ & 0.9900 \\
\hline$C(4)-C(5)$ & $1.518(4)$ & $\mathrm{C}(21)-\mathrm{H}(21 \mathrm{~B})$ & 0.9900 \\
\hline $\mathrm{C}(4)-\mathrm{H}(4)$ & 1.0000 & $C(22)-C(23)$ & $1.528(4)$ \\
\hline $\mathrm{C}(5)-\mathrm{H}(5 \mathrm{~A})$ & 0.9800 & $\mathrm{C}(22)-\mathrm{H}(22 \mathrm{~A})$ & 0.9900 \\
\hline $\mathrm{C}(5)-\mathrm{H}(5 \mathrm{~B})$ & 0.9800 & $\mathrm{C}(22)-\mathrm{H}(22 \mathrm{~B})$ & 0.9900 \\
\hline $\mathrm{C}(5)-\mathrm{H}(5 \mathrm{C})$ & 0.9800 & $\mathrm{C}(23)-\mathrm{H}(23 \mathrm{~A})$ & 0.9800 \\
\hline$C(7)-C(12)$ & $1.392(4)$ & $\mathrm{C}(23)-\mathrm{H}(23 \mathrm{~B})$ & 0.9800 \\
\hline$C(7)-C(8)$ & $1.395(4)$ & $\mathrm{C}(23)-\mathrm{H}(23 \mathrm{C})$ & 0.9800 \\
\hline $\mathrm{C}(8)-\mathrm{C}(9)$ & $1.378(4)$ & & \\
\hline $\mathrm{C}(8)-\mathrm{H}(8)$ & 0.9500 & $\mathrm{O}(2)-\mathrm{S}(1)-\mathrm{O}(3)$ & 119.97(13) \\
\hline $\mathrm{C}(9)-\mathrm{C}(10)$ & $1.403(4)$ & $\mathrm{O}(2)-\mathrm{S}(1)-\mathrm{N}(1)$ & $106.80(12)$ \\
\hline $\mathrm{C}(9)-\mathrm{H}(9)$ & 0.9500 & $\mathrm{O}(3)-\mathrm{S}(1)-\mathrm{N}(1)$ & $106.47(12)$ \\
\hline$C(10)-C(11)$ & $1.400(4)$ & $\mathrm{O}(2)-\mathrm{S}(1)-\mathrm{C}(7)$ & $107.50(13)$ \\
\hline$C(10)-C(13)$ & $1.507(4)$ & $\mathrm{O}(3)-\mathrm{S}(1)-\mathrm{C}(7)$ & $107.59(13)$ \\
\hline$C(11)-C(12)$ & $1.382(4)$ & $\mathrm{N}(1)-\mathrm{S}(1)-\mathrm{C}(7)$ & $108.01(12)$ \\
\hline $\mathrm{C}(11)-\mathrm{H}(11)$ & 0.9500 & $\mathrm{O}(1)-\mathrm{C}(1)-\mathrm{N}(1)$ & $112.7(2)$ \\
\hline $\mathrm{C}(12)-\mathrm{H}(12)$ & 0.9500 & $\mathrm{O}(1)-\mathrm{C}(1)-\mathrm{C}(14)$ & $112.6(2)$ \\
\hline $\mathrm{C}(13)-\mathrm{H}(13 \mathrm{~A})$ & 0.9800 & $\mathrm{~N}(1)-\mathrm{C}(1)-\mathrm{C}(14)$ & $111.3(2)$ \\
\hline $\mathrm{C}(13)-\mathrm{H}(13 \mathrm{~B})$ & 0.9800 & $\mathrm{O}(1)-\mathrm{C}(1)-\mathrm{H}(1)$ & 106.6 \\
\hline
\end{tabular}




\begin{tabular}{|c|c|c|c|}
\hline $\mathrm{N}(1)-\mathrm{C}(1)-\mathrm{H}(1)$ & 106.6 & $\mathrm{C}(8)-\mathrm{C}(9)-\mathrm{C}(10)$ & 121.1(3) \\
\hline $\mathrm{C}(14)-\mathrm{C}(1)-\mathrm{H}(1)$ & 106.6 & $\mathrm{C}(8)-\mathrm{C}(9)-\mathrm{H}(9)$ & 119.5 \\
\hline $\mathrm{C}(1)-\mathrm{N}(1)-\mathrm{C}(2)$ & $117.2(2)$ & $\mathrm{C}(10)-\mathrm{C}(9)-\mathrm{H}(9)$ & 119.5 \\
\hline $\mathrm{C}(1)-\mathrm{N}(1)-\mathrm{S}(1)$ & $118.42(19)$ & $\mathrm{C}(11)-\mathrm{C}(10)-\mathrm{C}(9)$ & 118.1(3) \\
\hline $\mathrm{C}(2)-\mathrm{N}(1)-\mathrm{S}(1)$ & $117.35(18)$ & $C(11)-C(10)-C(13)$ & $121.1(3)$ \\
\hline $\mathrm{C}(1)-\mathrm{O}(1)-\mathrm{C}(4)$ & $114.2(2)$ & $C(9)-C(10)-C(13)$ & $120.7(3)$ \\
\hline $\mathrm{N}(1)-\mathrm{C}(2)-\mathrm{C}(21)$ & $110.1(2)$ & $\mathrm{C}(12)-\mathrm{C}(11)-\mathrm{C}(10)$ & 121.2(3) \\
\hline $\mathrm{N}(1)-\mathrm{C}(2)-\mathrm{C}(3)$ & $109.5(2)$ & $\mathrm{C}(12)-\mathrm{C}(11)-\mathrm{H}(11)$ & 119.4 \\
\hline $\mathrm{C}(21)-\mathrm{C}(2)-\mathrm{C}(3)$ & $114.7(2)$ & $\mathrm{C}(10)-\mathrm{C}(11)-\mathrm{H}(11)$ & 119.4 \\
\hline $\mathrm{N}(1)-\mathrm{C}(2)-\mathrm{H}(2)$ & 107.4 & $\mathrm{C}(11)-\mathrm{C}(12)-\mathrm{C}(7)$ & $119.7(3)$ \\
\hline $\mathrm{C}(21)-\mathrm{C}(2)-\mathrm{H}(2)$ & 107.4 & $\mathrm{C}(11)-\mathrm{C}(12)-\mathrm{H}(12)$ & 120.2 \\
\hline $\mathrm{C}(3)-\mathrm{C}(2)-\mathrm{H}(2)$ & 107.4 & $\mathrm{C}(7)-\mathrm{C}(12)-\mathrm{H}(12)$ & 120.2 \\
\hline $\mathrm{C}(4)-\mathrm{C}(3)-\mathrm{C}(2)$ & $112.0(2)$ & $\mathrm{C}(10)-\mathrm{C}(13)-\mathrm{H}(13 \mathrm{~A})$ & 109.5 \\
\hline $\mathrm{C}(4)-\mathrm{C}(3)-\mathrm{H}(3 \mathrm{~A})$ & 109.2 & $\mathrm{C}(10)-\mathrm{C}(13)-\mathrm{H}(13 \mathrm{~B})$ & 109.5 \\
\hline $\mathrm{C}(2)-\mathrm{C}(3)-\mathrm{H}(3 \mathrm{~A})$ & 109.2 & $\mathrm{H}(13 \mathrm{~A})-\mathrm{C}(13)-\mathrm{H}(13 \mathrm{~B})$ & 109.5 \\
\hline $\mathrm{C}(4)-\mathrm{C}(3)-\mathrm{H}(3 \mathrm{~B})$ & 109.2 & $\mathrm{C}(10)-\mathrm{C}(13)-\mathrm{H}(13 \mathrm{C})$ & 109.5 \\
\hline $\mathrm{C}(2)-\mathrm{C}(3)-\mathrm{H}(3 \mathrm{~B})$ & 109.2 & $\mathrm{H}(13 \mathrm{~A})-\mathrm{C}(13)-\mathrm{H}(13 \mathrm{C})$ & 109.5 \\
\hline $\mathrm{H}(3 \mathrm{~A})-\mathrm{C}(3)-\mathrm{H}(3 \mathrm{~B})$ & 107.9 & $\mathrm{H}(13 \mathrm{~B})-\mathrm{C}(13)-\mathrm{H}(13 \mathrm{C})$ & 109.5 \\
\hline $\mathrm{O}(1)-\mathrm{C}(4)-\mathrm{C}(3)$ & $108.7(2)$ & $\mathrm{C}(19)-\mathrm{C}(14)-\mathrm{C}(15)$ & $119.2(2)$ \\
\hline $\mathrm{O}(1)-\mathrm{C}(4)-\mathrm{C}(5)$ & $105.4(2)$ & $\mathrm{C}(19)-\mathrm{C}(14)-\mathrm{C}(1)$ & $121.5(2)$ \\
\hline$C(3)-C(4)-C(5)$ & $114.2(2)$ & $\mathrm{C}(15)-\mathrm{C}(14)-\mathrm{C}(1)$ & 119.2(3) \\
\hline $\mathrm{O}(1)-\mathrm{C}(4)-\mathrm{H}(4)$ & 109.5 & $C(16)-C(15)-C(14)$ & $121.0(3)$ \\
\hline $\mathrm{C}(3)-\mathrm{C}(4)-\mathrm{H}(4)$ & 109.5 & $\mathrm{C}(16)-\mathrm{C}(15)-\mathrm{H}(15)$ & 119.5 \\
\hline $\mathrm{C}(5)-\mathrm{C}(4)-\mathrm{H}(4)$ & 109.5 & $\mathrm{C}(14)-\mathrm{C}(15)-\mathrm{H}(15)$ & 119.5 \\
\hline $\mathrm{C}(4)-\mathrm{C}(5)-\mathrm{H}(5 \mathrm{~A})$ & 109.5 & $C(15)-C(16)-C(17)$ & $118.4(3)$ \\
\hline $\mathrm{C}(4)-\mathrm{C}(5)-\mathrm{H}(5 \mathrm{~B})$ & 109.5 & $\mathrm{C}(15)-\mathrm{C}(16)-\mathrm{H}(16)$ & 120.8 \\
\hline $\mathrm{H}(5 \mathrm{~A})-\mathrm{C}(5)-\mathrm{H}(5 \mathrm{~B})$ & 109.5 & $\mathrm{C}(17)-\mathrm{C}(16)-\mathrm{H}(16)$ & 120.8 \\
\hline $\mathrm{C}(4)-\mathrm{C}(5)-\mathrm{H}(5 \mathrm{C})$ & 109.5 & $\mathrm{C}(16)-\mathrm{C}(17)-\mathrm{C}(18)$ & $122.3(3)$ \\
\hline $\mathrm{H}(5 \mathrm{~A})-\mathrm{C}(5)-\mathrm{H}(5 \mathrm{C})$ & 109.5 & $C(16)-C(17)-N(17)$ & $118.5(2)$ \\
\hline $\mathrm{H}(5 \mathrm{~B})-\mathrm{C}(5)-\mathrm{H}(5 \mathrm{C})$ & 109.5 & $\mathrm{C}(18)-\mathrm{C}(17)-\mathrm{N}(17)$ & $119.3(3)$ \\
\hline $\mathrm{C}(12)-\mathrm{C}(7)-\mathrm{C}(8)$ & $120.1(2)$ & $\mathrm{O}(18)-\mathrm{N}(17)-\mathrm{O}(17)$ & $123.5(2)$ \\
\hline $\mathrm{C}(12)-\mathrm{C}(7)-\mathrm{S}(1)$ & $120.6(2)$ & $\mathrm{O}(18)-\mathrm{N}(17)-\mathrm{C}(17)$ & $118.1(2)$ \\
\hline $\mathrm{C}(8)-\mathrm{C}(7)-\mathrm{S}(1)$ & $119.3(2)$ & $\mathrm{O}(17)-\mathrm{N}(17)-\mathrm{C}(17)$ & $118.4(3)$ \\
\hline $\mathrm{C}(9)-\mathrm{C}(8)-\mathrm{C}(7)$ & $119.8(3)$ & $C(19)-C(18)-C(17)$ & $118.4(3)$ \\
\hline $\mathrm{C}(9)-\mathrm{C}(8)-\mathrm{H}(8)$ & 120.1 & $\mathrm{C}(19)-\mathrm{C}(18)-\mathrm{H}(18)$ & 120.8 \\
\hline $\mathrm{C}(7)-\mathrm{C}(8)-\mathrm{H}(8)$ & 120.1 & $\mathrm{C}(17)-\mathrm{C}(18)-\mathrm{H}(18)$ & 120.8 \\
\hline
\end{tabular}




$\begin{array}{llll}\mathrm{C}(18)-\mathrm{C}(19)-\mathrm{C}(14) & 120.8(3) & \mathrm{C}(21)-\mathrm{C}(22)-\mathrm{H}(22 \mathrm{~A}) & 109.0 \\ \mathrm{C}(18)-\mathrm{C}(19)-\mathrm{H}(19) & 119.6 & \mathrm{C}(23)-\mathrm{C}(22)-\mathrm{H}(22 \mathrm{~B}) & 109.0 \\ \mathrm{C}(14)-\mathrm{C}(19)-\mathrm{H}(19) & 119.6 & \mathrm{C}(21)-\mathrm{C}(22)-\mathrm{H}(22 \mathrm{~B}) & 109.0 \\ \mathrm{C}(2)-\mathrm{C}(21)-\mathrm{C}(22) & 113.1(2) & \mathrm{H}(22 \mathrm{~A})-\mathrm{C}(22)-\mathrm{H}(22 \mathrm{~B}) & 107.8 \\ \mathrm{C}(2)-\mathrm{C}(21)-\mathrm{H}(21 \mathrm{~A}) & 109.0 & \mathrm{C}(22)-\mathrm{C}(23)-\mathrm{H}(23 \mathrm{~A}) & 109.5 \\ \mathrm{C}(22)-\mathrm{C}(21)-\mathrm{H}(21 \mathrm{~A}) & 109.0 & \mathrm{C}(22)-\mathrm{C}(23)-\mathrm{H}(23 \mathrm{~B}) & 109.5 \\ \mathrm{C}(2)-\mathrm{C}(21)-\mathrm{H}(21 \mathrm{~B}) & 109.0 & \mathrm{H}(23 \mathrm{~A})-\mathrm{C}(23)-\mathrm{H}(23 \mathrm{~B}) & 109.5 \\ \mathrm{C}(22)-\mathrm{C}(21)-\mathrm{H}(21 \mathrm{~B}) & 109.0 & \mathrm{C}(22)-\mathrm{C}(23)-\mathrm{H}(23 \mathrm{C}) & 109.5 \\ \mathrm{H}(21 \mathrm{~A})-\mathrm{C}(21)-\mathrm{H}(21 \mathrm{~B}) & 107.8 & \mathrm{H}(23 \mathrm{~A})-\mathrm{C}(23)-\mathrm{H}(23 \mathrm{C}) & 109.5 \\ \mathrm{C}(23)-\mathrm{C}(22)-\mathrm{C}(21) & 112.8(2) & \mathrm{H}(23 \mathrm{~B})-\mathrm{C}(23)-\mathrm{H}(23 \mathrm{C}) & 109.5 \\ \mathrm{C}(23)-\mathrm{C}(22)-\mathrm{H}(22 \mathrm{~A}) & 109.0 & & \end{array}$

Symmetry transformations used to generate equivalent atoms:

Table S18. Anisotropic displacement parameters $\left(\AA^{2} \times 10^{3}\right)$ for JF2976KTW42. The anisotropic displacement factor exponent takes the form: $-2 \pi^{2}\left[\mathrm{~h}^{2} \mathrm{a}^{* 2} \mathrm{U}^{11}+\ldots+2 \mathrm{~h} \mathrm{k} \mathrm{a}^{*} \mathrm{~b}^{*}\right.$ $\left.\mathrm{U}^{12}\right]$

\begin{tabular}{lcccccc}
\hline \multicolumn{2}{l}{$\mathrm{U}^{11}$} & \multicolumn{2}{l}{$\mathrm{U}^{22}$} & \multicolumn{2}{l}{$\mathrm{U}^{33}$} & \multicolumn{2}{l}{$\mathrm{U}^{23}$} & \multicolumn{2}{l}{$\mathrm{U}^{12}$} \\
$\mathrm{~S}(1)$ & $18(1)$ & $26(1)$ & $20(1)$ & $0(1)$ & $-1(1)$ & $3(1)$ \\
$\mathrm{C}(1)$ & $18(2)$ & $29(2)$ & $21(1)$ & $-1(1)$ & $-1(1)$ & $-1(1)$ \\
$\mathrm{N}(1)$ & $15(1)$ & $23(1)$ & $22(1)$ & $2(1)$ & $-2(1)$ & $0(1)$ \\
$\mathrm{O}(1)$ & $21(1)$ & $25(1)$ & $23(1)$ & $-2(1)$ & $0(1)$ & $-1(1)$ \\
$\mathrm{C}(2)$ & $17(2)$ & $23(2)$ & $23(1)$ & $0(1)$ & $-1(1)$ & $-1(1)$ \\
$\mathrm{O}(2)$ & $29(1)$ & $27(1)$ & $25(1)$ & $-2(1)$ & $-3(1)$ & $6(1)$ \\
$\mathrm{C}(3)$ & $18(2)$ & $33(2)$ & $21(1)$ & $2(1)$ & $0(1)$ & $0(1)$ \\
$\mathrm{O}(3)$ & $18(1)$ & $41(1)$ & $25(1)$ & $1(1)$ & $2(1)$ & $2(1)$ \\
$\mathrm{C}(4)$ & $19(2)$ & $28(2)$ & $21(1)$ & $0(1)$ & $0(1)$ & $1(1)$ \\
$\mathrm{C}(5)$ & $32(2)$ & $30(2)$ & $28(2)$ & $-1(1)$ & $3(1)$ & $3(2)$ \\
$\mathrm{C}(7)$ & $18(2)$ & $27(2)$ & $21(1)$ & $-1(1)$ & $-1(1)$ & $6(1)$ \\
$\mathrm{C}(8)$ & $20(2)$ & $24(2)$ & $25(2)$ & $3(1)$ & $-1(1)$ & $2(1)$ \\
$\mathrm{C}(9)$ & $23(2)$ & $20(2)$ & $28(2)$ & $-2(1)$ & $-5(1)$ & $1(1)$ \\
$\mathrm{C}(10)$ & $19(2)$ & $27(2)$ & $24(2)$ & $2(1)$ & $-3(1)$ & $5(1)$ \\
$\mathrm{C}(11)$ & $19(2)$ & $30(2)$ & $27(2)$ & $7(1)$ & $0(1)$ & $-2(1)$ \\
$\mathrm{C}(12)$ & $18(2)$ & $28(2)$ & $25(2)$ & $1(1)$ & $-3(1)$ & $0(1)$ \\
$\mathrm{C}(13)$ & $33(2)$ & $34(2)$ & $27(2)$ & $1(1)$ & $-1(1)$ & $4(2)$ \\
& & & & & &
\end{tabular}




\begin{tabular}{lllllll}
$\mathrm{C}(14)$ & $16(1)$ & $25(2)$ & $24(1)$ & $1(1)$ & $1(1)$ & $-4(1)$ \\
$\mathrm{C}(15)$ & $21(2)$ & $25(2)$ & $24(1)$ & $3(1)$ & $2(1)$ & $2(1)$ \\
$\mathrm{C}(16)$ & $23(2)$ & $27(2)$ & $24(2)$ & $0(1)$ & $6(1)$ & $1(1)$ \\
$\mathrm{C}(17)$ & $20(2)$ & $26(2)$ & $23(1)$ & $3(1)$ & $1(1)$ & $-2(1)$ \\
$\mathrm{N}(17)$ & $26(1)$ & $35(2)$ & $22(1)$ & $3(1)$ & $1(1)$ & $3(1)$ \\
$\mathrm{O}(17)$ & $44(1)$ & $41(1)$ & $20(1)$ & $-3(1)$ & $3(1)$ & $7(1)$ \\
$\mathrm{C}(18)$ & $22(2)$ & $20(2)$ & $27(2)$ & $2(1)$ & $-1(1)$ & $-2(1)$ \\
$\mathrm{O}(18)$ & $76(2)$ & $40(2)$ & $26(1)$ & $5(1)$ & $-2(1)$ & $23(1)$ \\
$\mathrm{C}(19)$ & $23(2)$ & $29(2)$ & $20(1)$ & $-3(1)$ & $1(1)$ & $-2(1)$ \\
$\mathrm{C}(21)$ & $18(1)$ & $26(2)$ & $23(1)$ & $1(1)$ & $-1(1)$ & $-2(1)$ \\
$\mathrm{C}(22)$ & $21(2)$ & $29(2)$ & $25(2)$ & $0(1)$ & $-4(1)$ & $-2(1)$ \\
$\mathrm{C}(23)$ & $27(2)$ & $32(2)$ & $23(2)$ & $-2(1)$ & $-2(1)$ & $0(1)$ \\
& & & & & & \\
\hline
\end{tabular}


Table S19. Hydrogen coordinates ( $\left.\times 10^{4}\right)$ and isotropic displacement parameters $\left(\AA^{2} \times 10^{3}\right)$ for JF2976KTW42.

\begin{tabular}{|c|c|c|c|c|}
\hline & $\mathrm{x}$ & $\mathrm{y}$ & z & $\mathrm{U}(\mathrm{eq})$ \\
\hline $\mathrm{H}(1)$ & 5336 & 5331 & 6178 & 27 \\
\hline $\mathrm{H}(2)$ & 8711 & 7078 & 6016 & 25 \\
\hline $\mathrm{H}(3 \mathrm{~A})$ & 9175 & 6099 & 5529 & 29 \\
\hline $\mathrm{H}(3 \mathrm{~B})$ & 10871 & 6095 & 5819 & 29 \\
\hline $\mathrm{H}(4)$ & 9835 & 4995 & 6208 & 27 \\
\hline $\mathrm{H}(5 \mathrm{~A})$ & 9868 & 4003 & 5684 & 45 \\
\hline $\mathrm{H}(5 \mathrm{~B})$ & 11288 & 4648 & 5555 & 45 \\
\hline $\mathrm{H}(5 \mathrm{C})$ & 9471 & 4636 & 5315 & 45 \\
\hline $\mathrm{H}(8)$ & 4066 & 5744 & 5396 & 28 \\
\hline $\mathrm{H}(9)$ & 4214 & 5615 & 4656 & 28 \\
\hline $\mathrm{H}(11)$ & 7150 & 7601 & 4591 & 31 \\
\hline $\mathrm{H}(12)$ & 6994 & 7737 & 5331 & 29 \\
\hline $\mathrm{H}(13 \mathrm{~A})$ & 6436 & 6952 & 3942 & 46 \\
\hline $\mathrm{H}(13 \mathrm{~B})$ & 4684 & 6454 & 3945 & 46 \\
\hline $\mathrm{H}(13 \mathrm{C})$ & 6486 & 6007 & 3997 & 46 \\
\hline $\mathrm{H}(15)$ & 5767 & 6057 & 6987 & 28 \\
\hline $\mathrm{H}(16)$ & 6186 & 5624 & 7690 & 30 \\
\hline $\mathrm{H}(18)$ & 8403 & 3602 & 7240 & 27 \\
\hline $\mathrm{H}(19)$ & 7997 & 4051 & 6538 & 29 \\
\hline $\mathrm{H}(21 \mathrm{~A})$ & 10873 & 6705 & 6529 & 27 \\
\hline $\mathrm{H}(21 \mathrm{~B})$ & 9550 & 6076 & 6726 & 27 \\
\hline $\mathrm{H}(22 \mathrm{~A})$ & 9188 & 7768 & 6746 & 30 \\
\hline $\mathrm{H}(22 \mathrm{~B})$ & 7617 & 7196 & 6865 & 30 \\
\hline $\mathrm{H}(23 \mathrm{~A})$ & 9111 & 6701 & 7459 & 41 \\
\hline $\mathrm{H}(23 \mathrm{~B})$ & 9112 & 7649 & 7489 & 41 \\
\hline $\mathrm{H}(23 \mathrm{C})$ & 10793 & 7190 & 7327 & 41 \\
\hline
\end{tabular}


Table S20. Torsion angles [ $\left.{ }^{\circ}\right]$ for JF2976KTW42.

\begin{tabular}{|c|c|c|c|}
\hline $\mathrm{O}(1)-\mathrm{C}(1)-\mathrm{N}(1)-\mathrm{C}(2)$ & $-45.0(3)$ & $\mathrm{C}(7)-\mathrm{C}(8)-\mathrm{C}(9)-\mathrm{C}(10)$ & $0.9(4)$ \\
\hline $\mathrm{C}(14)-\mathrm{C}(1)-\mathrm{N}(1)-\mathrm{C}(2)$ & $82.7(3)$ & $\mathrm{C}(8)-\mathrm{C}(9)-\mathrm{C}(10)-\mathrm{C}(11)$ & $-1.5(4)$ \\
\hline $\mathrm{O}(1)-\mathrm{C}(1)-\mathrm{N}(1)-\mathrm{S}(1)$ & $105.0(2)$ & $\mathrm{C}(8)-\mathrm{C}(9)-\mathrm{C}(10)-\mathrm{C}(13)$ & $179.4(3)$ \\
\hline $\mathrm{C}(14)-\mathrm{C}(1)-\mathrm{N}(1)-\mathrm{S}(1)$ & $-127.4(2)$ & $C(9)-C(10)-C(11)-C(12)$ & $1.4(4)$ \\
\hline $\mathrm{O}(2)-\mathrm{S}(1)-\mathrm{N}(1)-\mathrm{C}(1)$ & $164.68(18)$ & $\mathrm{C}(13)-\mathrm{C}(10)-\mathrm{C}(11)-\mathrm{C}(12)$ & $-179.6(3)$ \\
\hline $\mathrm{O}(3)-\mathrm{S}(1)-\mathrm{N}(1)-\mathrm{C}(1)$ & $35.4(2)$ & $\mathrm{C}(10)-\mathrm{C}(11)-\mathrm{C}(12)-\mathrm{C}(7)$ & $-0.5(4)$ \\
\hline $\mathrm{C}(7)-\mathrm{S}(1)-\mathrm{N}(1)-\mathrm{C}(1)$ & $-79.9(2)$ & $\mathrm{C}(8)-\mathrm{C}(7)-\mathrm{C}(12)-\mathrm{C}(11)$ & $-0.2(4)$ \\
\hline $\mathrm{O}(2)-\mathrm{S}(1)-\mathrm{N}(1)-\mathrm{C}(2)$ & $-45.4(2)$ & $S(1)-C(7)-C(12)-C(11)$ & $179.0(2)$ \\
\hline $\mathrm{O}(3)-\mathrm{S}(1)-\mathrm{N}(1)-\mathrm{C}(2)$ & $-174.75(18)$ & $\mathrm{O}(1)-\mathrm{C}(1)-\mathrm{C}(14)-\mathrm{C}(19)$ & $-14.5(4)$ \\
\hline$C(7)-S(1)-N(1)-C(2)$ & $69.9(2)$ & $\mathrm{N}(1)-\mathrm{C}(1)-\mathrm{C}(14)-\mathrm{C}(19)$ & $-142.2(3)$ \\
\hline $\mathrm{N}(1)-\mathrm{C}(1)-\mathrm{O}(1)-\mathrm{C}(4)$ & $53.6(3)$ & $\mathrm{O}(1)-\mathrm{C}(1)-\mathrm{C}(14)-\mathrm{C}(15)$ & $169.6(2)$ \\
\hline $\mathrm{C}(14)-\mathrm{C}(1)-\mathrm{O}(1)-\mathrm{C}(4)$ & $-73.4(3)$ & $\mathrm{N}(1)-\mathrm{C}(1)-\mathrm{C}(14)-\mathrm{C}(15)$ & $41.9(3)$ \\
\hline $\mathrm{C}(1)-\mathrm{N}(1)-\mathrm{C}(2)-\mathrm{C}(21)$ & $-84.7(3)$ & $C(19)-C(14)-C(15)-C(16)$ & $3.0(4)$ \\
\hline $\mathrm{S}(1)-\mathrm{N}(1)-\mathrm{C}(2)-\mathrm{C}(21)$ & $125.1(2)$ & $\mathrm{C}(1)-\mathrm{C}(14)-\mathrm{C}(15)-\mathrm{C}(16)$ & $179.0(3)$ \\
\hline $\mathrm{C}(1)-\mathrm{N}(1)-\mathrm{C}(2)-\mathrm{C}(3)$ & $42.4(3)$ & $C(14)-C(15)-C(16)-C(17)$ & $-0.9(4)$ \\
\hline $\mathrm{S}(1)-\mathrm{N}(1)-\mathrm{C}(2)-\mathrm{C}(3)$ & $-107.9(2)$ & $\mathrm{C}(15)-\mathrm{C}(16)-\mathrm{C}(17)-\mathrm{C}(18)$ & $-1.6(4)$ \\
\hline $\mathrm{N}(1)-\mathrm{C}(2)-\mathrm{C}(3)-\mathrm{C}(4)$ & $-48.9(3)$ & $\mathrm{C}(15)-\mathrm{C}(16)-\mathrm{C}(17)-\mathrm{N}(17)$ & $178.9(3)$ \\
\hline$C(21)-C(2)-C(3)-C(4)$ & $75.5(3)$ & $\mathrm{C}(16)-\mathrm{C}(17)-\mathrm{N}(17)-\mathrm{O}(18)$ & $171.9(3)$ \\
\hline $\mathrm{C}(1)-\mathrm{O}(1)-\mathrm{C}(4)-\mathrm{C}(3)$ & $-60.6(3)$ & $\mathrm{C}(18)-\mathrm{C}(17)-\mathrm{N}(17)-\mathrm{O}(18)$ & $-7.5(4)$ \\
\hline $\mathrm{C}(1)-\mathrm{O}(1)-\mathrm{C}(4)-\mathrm{C}(5)$ & $176.5(2)$ & $\mathrm{C}(16)-\mathrm{C}(17)-\mathrm{N}(17)-\mathrm{O}(17)$ & $-8.6(4)$ \\
\hline $\mathrm{C}(2)-\mathrm{C}(3)-\mathrm{C}(4)-\mathrm{O}(1)$ & $57.8(3)$ & $\mathrm{C}(18)-\mathrm{C}(17)-\mathrm{N}(17)-\mathrm{O}(17)$ & $171.9(3)$ \\
\hline$C(2)-C(3)-C(4)-C(5)$ & $175.1(2)$ & $\mathrm{C}(16)-\mathrm{C}(17)-\mathrm{C}(18)-\mathrm{C}(19)$ & $1.9(4)$ \\
\hline $\mathrm{O}(2)-\mathrm{S}(1)-\mathrm{C}(7)-\mathrm{C}(12)$ & $19.7(3)$ & $\mathrm{N}(17)-\mathrm{C}(17)-\mathrm{C}(18)-\mathrm{C}(19)$ & $-178.6(3)$ \\
\hline $\mathrm{O}(3)-\mathrm{S}(1)-\mathrm{C}(7)-\mathrm{C}(12)$ & $150.2(2)$ & $C(17)-C(18)-C(19)-C(14)$ & $0.3(4)$ \\
\hline $\mathrm{N}(1)-\mathrm{S}(1)-\mathrm{C}(7)-\mathrm{C}(12)$ & $-95.3(2)$ & $C(15)-C(14)-C(19)-C(18)$ & $-2.7(4)$ \\
\hline $\mathrm{O}(2)-\mathrm{S}(1)-\mathrm{C}(7)-\mathrm{C}(8)$ & $-161.2(2)$ & $\mathrm{C}(1)-\mathrm{C}(14)-\mathrm{C}(19)-\mathrm{C}(18)$ & $-178.6(3)$ \\
\hline $\mathrm{O}(3)-\mathrm{S}(1)-\mathrm{C}(7)-\mathrm{C}(8)$ & $-30.7(3)$ & $\mathrm{N}(1)-\mathrm{C}(2)-\mathrm{C}(21)-\mathrm{C}(22)$ & $-65.1(3)$ \\
\hline $\mathrm{N}(1)-\mathrm{S}(1)-\mathrm{C}(7)-\mathrm{C}(8)$ & $83.9(2)$ & $\mathrm{C}(3)-\mathrm{C}(2)-\mathrm{C}(21)-\mathrm{C}(22)$ & $170.9(2)$ \\
\hline $\mathrm{C}(12)-\mathrm{C}(7)-\mathrm{C}(8)-\mathrm{C}(9)$ & $0.0(4)$ & $\mathrm{C}(2)-\mathrm{C}(21)-\mathrm{C}(22)-\mathrm{C}(23)$ & $168.4(2)$ \\
\hline $\mathrm{S}(1)-\mathrm{C}(7)-\mathrm{C}(8)-\mathrm{C}(9)$ & $-179.2(2)$ & & \\
\hline
\end{tabular}

Symmetry transformations used to generate equivalent atoms: 
Table S21. Hydrogen bonds for JF2976KTW42 [Å and $\left.{ }^{\circ}\right]$.

\begin{tabular}{lcccc}
\hline D-H...A & d(D-H) & $d(\mathrm{H} \ldots \mathrm{A})$ & $\mathrm{d}(\mathrm{D} \ldots \mathrm{A})$ & $<(\mathrm{DHA})$ \\
\hline $\mathrm{C}(1)-\mathrm{H}(1) \ldots \mathrm{O}(3)$ & 1.00 & 2.32 & $2.870(4)$ & 114 \\
$\mathrm{C}(3)-\mathrm{H}(3 \mathrm{~B}) \ldots \mathrm{O}(3) \# 1$ & 0.99 & 2.57 & $3.503(4)$ & 157 \\
$\mathrm{C}(4)-\mathrm{H}(4) \ldots \mathrm{O}(17) \# 2$ & 1.00 & 2.59 & $3.520(3)$ & 154 \\
$\mathrm{C}(21)-\mathrm{H}(21 \mathrm{~A}) \ldots \mathrm{O}(3) \# 1$ & 0.99 & 2.61 & $3.525(3)$ & 153 \\
& & & & \\
\hline
\end{tabular}

Symmetry transformations used to generate equivalent atoms:

$\# 1 \mathrm{x}+1, \mathrm{y}, \mathrm{z} \quad \# 2 \mathrm{x}+1 / 2, \mathrm{y}, \mathrm{z}+3 / 2$

\section{References:}

1. Bruker (2014) APEX2 (Version 2014.9) and (2016) SAINT (Version 8.37a). Bruker AXS Inc., Madison, Wisconsin, USA.

2. An Empirical Correction for Absorption Anisotropy, Blessing, R. H. (1995). Acta Cryst., A51, 33-38.

3. Sheldrick, G.M., SADABS (2016) Version 2016/2, 'Siemens Area Detector Absorption Correction' Universität Göttingen: Göttingen, Germany.

4. Sheldrick, G.M., (2002). SHELXTL. Version 6.1. Bruker AXS Inc., Madison, Wisconsin, USA.

5. Sheldrick, G. M., (2015) SHELXT-Integrated Space-Group and Crystal-Structure Determination. Acta Crystallographica Section A, A71, 3-8.

6. Sheldrick, G. M., (2017). SHELXL2017/1. Universität Göttingen: Göttingen, Germany. 